\title{
Conceptual Design for a Laminar-Flying-Wing Aircraft
}

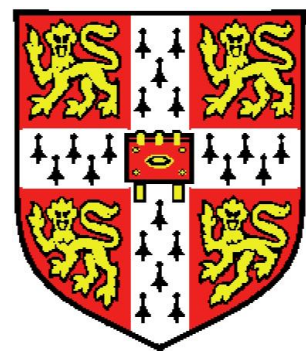

\author{
Tariq Issam Saeed \\ Trinity Hall \\ University of Cambridge
}

A thesis submitted for the degree of

Doctor of Philosophy

February 2012 



\section{Declaration}

Described in this dissertation is worked performed in the Department of Engineering at the University of Cambridge between October 2008 and February 2012. I hereby declare that no part of this work has been submitted as an exercise for a degree at this or any other university. This dissertation is entirely the result of my own work and includes nothing which is the outcome of collaboration. This dissertation contains approximately 122 figures and 48,500 words.

Signature:

Date: 

This thesis is dedicated to my father, for his guidance and encouragement throughout my life, and my mother. 



\section{Acknowledgements}

Firstly, I would like to express my gratitude toward my supervisor Dr Will Graham, for sharing his knowledge of aerodynamics, and his unwavering support throughout. It has been a honour to work with him.

I would like to thank Prof Holger Babinsky for the useful discussions on the pressure drop across a porous skin, and his general advice. I must acknowledge Dr Chez Hall's contribution to this research through his derivation of the pump power; I am also indebted to him for the many useful discussions on aircraft jet propulsion. Thanks also go to Dr Keith Seffen for the useful discussions on structural design and Dr Jerome Jarrett for his general advice. Finally, I would like to acknowledge Jeremy Eastwood, Mudassir Lone, Charles Ni and Ian Vigrass for their contributions to the undergraduate 'Green Aircraft Design' project; I am especially grateful to Jeremy Eastwood for sharing with me his knowledge of shell scripting.

I must thank the UK Engineering and Physical Sciences Research Council for its financial support via its Doctoral Training scheme. In addition, I extend my gratitude to Trinity Hall for its financial assistance, which has helped facilitate my attendance at international conferences. 



\begin{abstract}
The laminar-flying-wing aircraft appears to be an attractive long-term prospect for reducing the environmental impact of commercial aviation. In assessing its potential, a relatively straightforward initial step is the conceptual design of a version with restricted sweep angle. Such a design is the topic of this thesis. In addition to boundary layer laminarisation (utilising distributed suction) and limited sweep, a standing-height passenger cabin and subcritical aerofoil flow are imposed as requirements. Subject to these constraints, this research aims to: provide insight into the parameters affecting practical laminar-flow-control suction power requirements; identify a viable basic design specification; and, on the basis of this, an assessment of the fuel efficiency through a detailed conceptual design study.

It is shown that there is a minimum power requirement independent of the suction system design, associated with the stagnation pressure loss in the boundary layer. This requirement increases with aerofoil section thickness, but depends only weakly on Mach number and (for a thick, lightly-loaded laminar flying wing) lift coefficient. Deviation from the optimal suction distribution, due to a practical chamber-based architecture, is found to have very little effect on the overall suction coefficient. In the spanwise direction, through suitable choice of chamber depth, the pressure drop due to frictional and inertial effects may be rendered negligible. Finally, it is found that the pressure drop from the aerofoil surface to the pump collector ducts determines the power penalty; suggesting there is little benefit in trying to maintain an optimal suction distribution through increased subsurface-chamber complexity. For representative parameter values, the minimum power associated with boundary-layer losses alone contributes some $80 \%$ $90 \%$ of the total power requirement.

To identify the viable basic design specification, a high-level exploration of the laminar-flying-wing design space is performed, with an emphasis above all on aerodynamic efficiency. The characteristics of the design are assessed as a function of three parameters: thickness-to-chord ratio, wingspan, and unit Reynolds
\end{abstract}


number. A feasible specification, with $20 \%$ thickness-to-chord, $80 \mathrm{~m}$ span and a unit Reynolds number of $8 \times 10^{6} \mathrm{~m}^{-1}$, is identified; it corresponds to a 187 tonne aircraft which cruises at Mach 0.67 and altitude 22,500 ft, with lift coefficient 0.14. The benefit of laminarisation is manifested in a high lift-to-drag ratio, but the wing loading is low, and the structural efficiency and gust response are thus likely to be relatively poor.

On the basis of this specification, a detailed conceptual design is undertaken. A 220-passenger laminar-flying-wing concept, propelled by three turboprop engines, with a cruise range of $9000 \mathrm{~km}$ is developed. The estimated fuel burn is 13.9 $\mathrm{g} /$ pax.km. For comparison, a conventional aircraft, propelled by four turboprop engines, with a high-mounted, unswept, wing is designed for the same mission specification and propulsion characteristics, and is shown to have a fuel burn of $15.0 \mathrm{~g} /$ pax.km. Despite significant aerodynamic efficiency gains, the fuel burn of the laminar flying wing is only marginally better as it suffers from a poor cruise engine efficiency, due to extreme differences between takeoff and cruising requirements, and is much heavier.

The laminar flying wing proposed in this thesis falls short of the performance improvements expected of the concept, and is not worth the development effort. It is therefore proposed that research efforts either be focussed on improving the engine efficiency, or switching to a low aspect ratio, high sweep, design configuration. 


\section{Publications}

1. Saeed, T.I., Graham, W.R., Babinsky, H., Eastwood, J.P., Hall, C.A., Jarrett, J.P, Lone, M. and Seffen, K.A., Conceptual Design for a Laminar Flying Wing Aircraft, 27th AIAA Applied Aerodynamics Conference, San Antonio, Texas, June 22-25, 2009, AIAA-2009-3616.

2. Saeed, T.I., Graham, W.R., Babinsky, H. and Hall, C.A., Boundary Layer Suction System Design for Application to Laminar Flying Wing Aircraft, 28th AIAA Applied Aerodynamics Conference, Chicago, Illinois, June 28-1, 2010, AIAA-2010-4379.

3. Saeed, T.I., Graham, W.R. and Hall, C.A., Boundary-Layer Suction System Design for Laminar-Flying-Wing Aircraft, Journal of Aircraft, Vol. 48, No. 4, 2011, pp. $1368-1379$.

4. Saeed, T.I. and Graham, W.R., Conceptual Design for a Laminar-FlyingWing Aircraft, 50th AIAA Aerospace Sciences and New Horizons Meeting, Nashville, Tennessee, January 9-12, 2012, AIAA-2012-0868. 



\section{Contents}

Contents $\quad$ xiii

List of Figures $\quad$ xix

List of Tables $\quad$ xxvii

$\begin{array}{ll}\text { Nomenclature } & \text { xxix }\end{array}$

1 Introduction 1

2 Literature Review $\quad \begin{aligned} & \mathbf{7} \\ & \end{aligned}$

2.1 Laminar Flow Control . . . . . . . . . . . . . . . . . 7

2.1.1 Profile Drag Reduction _. . . . . . . . . . . . . . 8

2.1.2 Flow Control Design Methodology . . . . . . . . . . . . . . . . . . . . . . . . . . . . 13

2.1 .3 Boundary Layer Stability . . . . . . . . . . . . . . . . . . . . 14

2.1.3.1 Tollmien-Schlichting Waves . . . . . . . . . . . . . . . . 14

2.1.3.2 Taylor-Görtler Vortices . . . . . . . . . . . . . . . . 14

2.1.3.3 Crossflow Instabilities . . . . . . . . . . . . . . . . . . . . . 14

2.1.3.4 Attachment-Line Instabilities . . . . . . . . . . . . . . 16

2.1.3.5 Shock Wave / Laminar Boundary Layer Interactions . . . . . 17

2.1.3.6 Example Suction Distributions . . . . . . . . . . . . 17

2.1.4 LFC System Design Considerations . . . . . . . . . . . . . . . . . . 19

2.2 The Flying Wing Configuration . . . . . . . . . . . . . . . . . 22

2.2.1 Structural Architecture . . . . . . . . . . . . . . . . 23

2.2.2 Cabin Arrangement and Operational aspects . . . . . . . . . . 25

2.2.3 Stability and Control Considerations . . . . . . . . . . . . . 27

2.2.3.1 Longitudinal Stability . . . . . . . . . . . . . . . 27

2.2.3.2 Lateral-Directional Stability . . . . . . . . . . . . . 29

2.2 .3 .3 Control . . . . . . . . . . . . . . . . . . 30 
2.2.3.4 Effects of Wing and Planform Geometry on Stability and Control ........................... 32

2.2.4 Flying and Handling Qualities . . . . . . . . . . . . . . . 35

2.2.4.1 Civil and Military Specifications . . . . . . . . . . 35

2.2.4.2 Behaviour of Tailless Aircraft . . . . . . . . . . . . 36

2.2 .5 Aeroelastic Behaviour . . . . . . . . . . . . . . 36

2.3 Laminar-Flying-Wing Design Concepts . . . . . . . . . . . . . . . . . . 37

2.3.1 Greener by Design . . . . . . . . . . . . . . . . . . . . 37

2.3.2 Delta-Wing Aircraft with Suction . . . . . . . . . . . . . . . . 38

2.3.3 The Handley Page HP117 Proposal . . . . . . . . . . . . . . . . . . . 40

2.4 Summary and Research Proposal . . . . . . . . . . . . . . . . . . . . . 42

3 Design Methodology 45

3.1 General Approach . . . . . . . . . . . . . . . . . . . . . 45

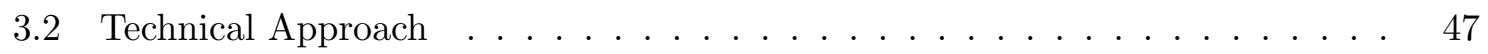

3.2.1 3-D Airframe Generation and Aircraft Performance . . . . . . . . . . . 47

3.2.1.1 Computational Tools . . . . . . . . . . . . . 47

3.2.1.2 Aircraft Profile Drag Estimation Method . . . . . . . . . 50

3.2 .2 Propulsion System Design _ . . . . . . . . . . . . . . . 57

3.2.2.1 Design Cycle Parameters . . . . . . . . . . . . 57

3.2 .2 .2 GasTurb . . . . . . . . . . . . . . 60

3.2 .3 Structures and Weights . . . . . . . . . . . . . 65

3.2.3.1 Methodology . . . . . . . . . . . . . . 65

3.2.3.2 Loading Conditions . . . . . . . . . . . . . . . 65

3.2.3.3 Fuselage Structure . . . . . . . . . . . . . . . . . . . . . . . . . . . . . . 67

3.2.3.4 Wing Structure . . . . . . . . . . . . . . 74

3.2.3.5 Validity of Design Methodology ............ 85

3.2 .4 Mission Analysis . . . . . . . . . . . . . . . . . . . . 90

4 Boundary-Layer Suction System Design $\quad 91$

4.1 Boundary-Layer Suction Calculation Method . . . . . . . . . . . . . . . 91

4.1 .1 Boundary Layer Solver . . . . . . . . . . . . . . . . . . . . 91

4.1.2 Suction Algorithm and Validation . . . . . . . . . . . . . . . . 94

4.2 Pump Power Analysis . . . . . . . . . . . . . . . . . . 97

4.3 Minimum Suction Requirements . . . . . . . . . . . . . . . . . 98

4.3 .1 Typical Suction and Power Distributions . . . . . . . . . . . . . 98 
4.3 .2 Parameter Study . . . . . . . . . . . . . . . . . . . . . 100

4.4 Suction Hardware Design and Power Consumption . . . . . . . . . . . . . . . 104

4.4 .1 Suction Chamber Layout . . . . . . . . . . . . . . . . . . . . . 104

4.4 .2 Skin Losses and Chamber Discretisation . . . . . . . . . . . . . . . . 105

4.4.2.1 Pressure Drop Across a Porous Skin . . . . . . . . . . . . . . 106

4.4.2.2 Chamber Specification Algorithm . . . . . . . . . . . . . 107

4.4.2.3 Chamber Constraint Trade-Off Study . . . . . . . . . . . . . 109

4.4 .3 Spanwise Chamber Losses . . . . . . . . . . . . . . . . . . . . . . 114

4.4 .4 Collector Duct Trade-Off Study . . . . . . . . . . . . . . . . . . . 116

4.5 Summary . . . . . . . . . . . . . . . . . . . . . . 118

5 LFW Aircraft and Mission Specification $\quad 121$

5.1 Design Approach . . . . . . . . . . . . . . . . . . . . . . . . . . . 121

5.1.1 Aircraft Configuration and Geometric Constraints . . . . . . . . . . . 122

5.1 .2 Aerodynamic Constraints . . . . . . . . . . . . . . . . . 123

5.1.2.1 Unit Reynolds Number . . . . . . . . . . . . . . . . . . 123

5.1.2.2 Planform Sweep . . . . . . . . . . . . . . . . . 123

5.1 .2 .3 Mach Number . . . . . . . . . . . . . . . . . . . . . . 124

5.1.3 Design Specification Algorithm . . . . . . . . . . . . . . . . . . 124

5.2 Methodology . . . . . . . . . . . . . . . . . . . 125

5.2 .1 Drag Polar . . . . . . . . . . . . . . . . . . 125

5.2 .2 Cruise Condition . . . . . . . . . . . . . . . . . 126

5.2 .3 Lift and Drag Calculations _. . . . . . . . . . . . . 126

5.3 Aircraft and Mission Parametric Study . . . . . . . . . . . . . . . . . . 128

5.3 .1 Aspect Ratio . . . . . . . . . . . . . . . . . . . . . 128

$5.3 .2 \quad$ Sweep . . . . . . . . . . . . . . . . . . . . . . . . . 128

5.3 .3 Profile Drag . . . . . . . . . . . . . . . . . . . . . 128

5.3.4 Cruise Lift Coefficient and Aerodynamic Efficiency . . . . . . . . . . . 129

5.3.5 Cruise Mach Number . . . . . . . . . . . . . . . . . . . . . . . 132

5.3 .6 Cruise Altitude and Velocity . . . . . . . . . . . . . . . 132

5.3.7 Wing Loading and Maximum Lift Coefficient . . . . . . . . . . . . . . 134

5.3.8 Maximum Cruise Weight and Structural Weight Fraction . . . . . . 135

5.4 Discussion . . . . . . . . . . . . . . . . . . . . . . . . 138

5.4.1 Mission Parameter Conflicts . . . . . . . . . . . . . . . . 138

5.4.2 Synthesis of Parameter Considerations . . . . . . . . . . . . . . . 139

5.4 .3 Sensitivity to Error . . . . . . . . . . . . . . . . . . . . . . 140 
5.4.4 Comparison with Conventional Aircraft . . . . . . . . . . . . . . . 141

5.5 Summary . . . . . . . . . . . . . . . . . . . . . . . 142

6 Conceptual Design Study $\quad 145$

6.1 Laminar-Flying-Wing Design Concept . . . . . . . . . . . . . . . . . . 145

6.1.1 Three-Dimensional Airframe Generation . . . . . . . . . . . . . . . 145

6.1.1.1 Flying and Handling Qualities Requirement . . . . . . . . . 146

6.1.1.2 Planform . . . . . . . . . . . . . . . . 146

6.1.1.3 Wing and Cabin Design . . . . . . . . . . . . . . 152

6.1.2 Aircraft Performance . . . . . . . . . . . . . . . . . 158

6.1.2.1 Methodology . . . . . . . . . . . . . . 158

6.1.2.2 Cruise Performance . . . . . . . . . . . . . . . . . 159

6.1.2.3 Low-Speed Performance . . . . . . . . . . . . . . . . . . 161

6.1.3 Suction-System Technology Integration . . . . . . . . . . . . . . 163

6.1.3.1 System Architecture . . . . . . . . . . . . . . . . . . . . 164

6.1.3.2 System Specification . . . . . . . . . . . . . . . . 165

6.1.3.3 Power Requirement and Weight Penalty . . . . . . . . . . . 169

6.1 .4 Propulsion System . . . . . . . . . . . . . . . . . . . . . . . . . . . . 169

6.1.4.1 Thrust and Power Requirements . . . . . . . . . . . . . . . 170

6.1.4.2 Power-Plant Scoping Study . . . . . . . . . . . . . . . . . . 171

6.1.4.3 LFW Engine Performance . . . . . . . . . . . . . . . 178

6.1.4.4 Sensitivity Study . . . . . . . . . . . . . . . . . . . . . 182

6.1.5 Structures and Weights . . . . . . . . . . . . . . . . . . 182

6.1.5.1 Structural Configuration . . . . . . . . . . . . . 183

6.1.5.2 System Loads Distribution . . . . . . . . . . . . . . . 185

6.1.5.3 Structural Analysis . . . . . . . . . . . . . . . . . 185

6.1.5.4 Weight Breakdown . . . . . . . . . . . . . . 189

6.1.5.5 Centre-of-Gravity Buildup and Landing Gear Arrangement . 189

6.1.6 Mission . . . . . . . . . . . . . . . . . . . . . . . . . . . . . 192

6.2 Competitor Aircraft Design Concept . . . . . . . . . . . . . . . . 193

6.2.1 Target Gross Weight . . . . . . . . . . . . . . . . . . . . . . 193

6.2 .2 Cruise Lift Coefficient . . . . . . . . . . . . . . . . . . . . 193

6.2 .3 Aircraft Geometry . . . . . . . . . . . . . . . . . . . . . . . . . . 194

6.2 .3 .1 Fuselage . . . . . . . . . . . . . . . . . . . 194

6.2 .3 .2 Wing ........................ 195

6.2.3.3 Tailplane . . . . . . . . . . . . . . . . . . . 195 
6.2.4 Aircraft Performance . . . . . . . . . . . . . . . . . . . . . . 198

6.2.4.1 Cruise Performance . . . . . . . . . . . . . . . . 198

6.2.4.2 Low-Speed Performance . . . . . . . . . . . . . . . . 199

6.2 .5 Propulsion System . . . . . . . . . . . . . . . . . . . . 200

6.2 .6 Structures and Weights _. . . . . . . . . . . . . . 201

6.2.6.1 Structural Weight . . . . . . . . . . . . . . . . 201

6.2 .6 .2 Fuel Weight . . . . . . . . . . . . . . . . . 203

6.2.6.3 Aircraft Weight Buildup and Centre-of-Gravity Travel . . . . 203

6.3 Comparison of LFW and Competitor Aircraft . . . . . . . . . . . . . . . . . . 204

6.4 Summary . . . . . . . . . . . . . . . . . . . 205

7 Conclusions and Future Work $\quad 207$

7.1 Conclusions . . . . . . . . . . . . . . . . . . . . 207

7.1.1 Motivation for the Study . . . . . . . . . . . . . . . . . 207

7.1 .2 Conclusions of the Study . . . . . . . . . . . . . . . . . 207

7.2 Recommendations for Future Work . . . . . . . . . . . . . . . . . . 210

$\begin{array}{ll}\text { References } & 213\end{array}$ 


\section{LIST OF FIGURES}

1.1 Conventional aircraft layout - the Boeing 787 Dreamliner. . . . . . . . . . 1

1.2 Engine fuel consumption and fuel burn per seat over a $1000 \mathrm{~nm}$ mission (from

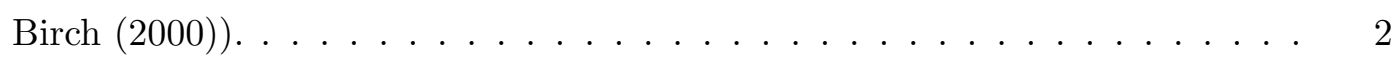

1.3 D8 aircraft series (from Greitzer et al. (2010)) . . . . . . . . . . . . . . . 2

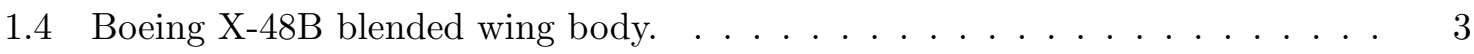

1.5 Northrop YRB-49 bomber. . . . . . . . . . . . . . . . . . . . 4

2.1 Stable and unstable velocity profiles in a flat-plate laminar boundary layer flat plate corresponds to zero-pressure gradient. (Taken from Green (2006).) • 8

2.2 Boundary layer development with suction (from Lachmann (1955)). . . . . . 9

2.3 Wake profile for an integrated propulsion and suction system (from Lee (1961)). 11

2.4 Variation in wing drag coefficient with chord Reynolds number for laminar boundary layers (from Denning et al. (1997)) . . . . . . . . . . . . . . 12

2.5 Block diagram of LFC design cycle (from Harris et al. (1988)). . . . . . . . . 13

2.6 Schematic diagram of Taylor-Görtler vortices (from Joslin (1998b)). . . . . . 14

2.7 Schematic diagram of the crossflow instability and velocity profile. . . . . . 15

2.8 Schematic diagram of attachment-line flow (from Joslin (1998b)) . . . . . . . 16

2.9 Example suction flow velocity distribution for the $30^{\circ}$ (quarter-chord) sweep X-21A aircraft (Joslin $(1998 \mathrm{a})) . \ldots \ldots \ldots$

2.10 Example suction flow velocity distribution for the $10^{\circ}$ (quarter-chord) sweep

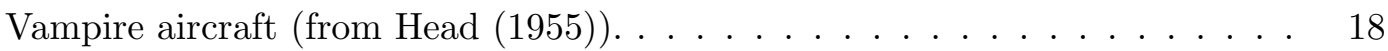

2.11 Outline for a laminar flow control system (from Edwards (1977)) . . . . . . . 19

2.12 Typical suction surface (from Edwards (1977)) . . . . . . . . . . . . . . 20

2.13 Various pressure loss components making up pump power (from Lachmann

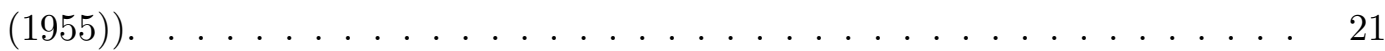

2.14 X-21A laminar flow pumping system (from Kosin (1965)). . . . . . . . . . . 22

2.15 Structural concepts for the centrebody pressure vessel (from Liebeck (2004)). 24

2.16 Cabin seating arrangement (from Martinez-Val (2007)) . . . . . . . . . . 25 
2.17 Differential elevon control for a pure aileron deflection to the right without any elevator effect (from Nickel and Wohlfart (1994)). . . . . . . . . . . . . . 30

2.18 B-2 split flap rudders. . . . . . . . . . . . . . . . . . . . . . . . 32

2.19 The lift distributions of two rectangular wings with an aspect ratio of 5 and sweep angles of $\phi=0^{\circ}$ and $\phi=45^{\circ}$. The lift distribution of the swept wing has been plotted for equal lift (solid line) and for the same angle of attack (dashed line). (Taken from Nickel and Wohlfart (1994)). . . . . . . . . . . . .

2.20 Laminarised aircraft — relative range performance — fixed integrated delta planforms. Assumptions: optimum $L / D$ cruise (no allowances), cruise Mach number of 0.8 , datum $C_{D 0}=0.010$ (no suction treatment), and $C_{Q}=0.0005$. (Taken from Denning et al. (1997)). . . . . . . . . . . . . . 39

2.21 Handley Page HP117 LFW configuration (from Green (2006)). . . . . . . . . 40

3.1 Schematic diagram of the design process envisaged for the LFW. . . . . . . 46

3.2 Wake streamline contraction due to fuselage thickness, carrying wing circulation into the wake (from Greitzer et al. (2010)) . . . . . . . . . . . . . 48

3.3 Example distribution of bound and trailing vortices (output from AVL). . . . 49

3.4 Schematic diagram of friction and pressure drag forces on an infinite sweptwing (Greitzer et al. (2010)). . . . . . . . . . . . . . . . . 50

3.5 Derivation of fuselage cross-flow drag (from Torenbeek (1976)) . . . . . . . . 53

3.6 Estimated fuselage profile drag increment due to angle of attack and rear fuselage upsweep (from Torenbeek (1976)). ( $\alpha_{f}^{\prime}$ demarcates the fuselage incidence at which the extra drag is a minimum for a given $\beta$ value.) . . . . . . . . 54

3.7 Profile drag increment due to surface roughness (from Torenbeek (1976)). . . 57

$3.845^{\circ}$ swept eight-bladed propeller map at $M_{\infty}=0.70$ (from Rohrbach et al.

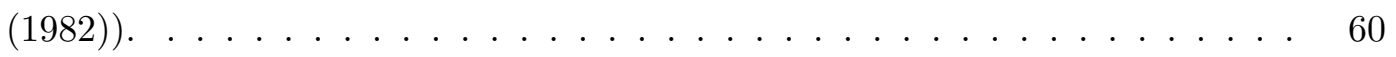

3.9 Compressor map scaling parameters (from GasTurb manual) . . . . . . . . 63

3.10 Schematic of structural design methodology. . . . . . . . . . . . . 66

3.11 Multi-bubble geometric parameters (perpendicular to the spanwise axis). . . 68

3.12 Cabin cross section (perpendicular to the chordwise axis) . . . . . . . . 69

3.13 Variation of the ratio of axial-to-hoopwise stress with $\theta_{f, v b}$ and $N$ for $\theta_{f, 1}=$

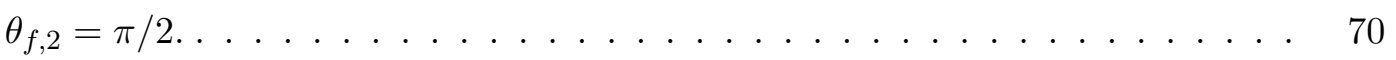

3.14 Vertical bulkhead force. . . . . . . . . . . . . . . . . . 70

3.15 Conventional fuselage geometry and applied loads (from Greitzer et al. (2010)). 73

3.16 Wing construction (from Howe (2004)) . . . . . . . . . . . . . . 74

3.17 Wing (or tail) planform. . . . . . . . . . . . . . . . 75 
3.18 Wing (or tail) aerofoil and wing-box cross section (perpendicular to the reference axis) . . . . . . . . . . . . . . . . . 76

3.19 Schematic diagram of rib flange and spar construction. . . . . . . . . . . . 79

3.20 Schematic diagram of rib arrangement and forces at hinge attachment point. $\quad 80$

3.21 Schematic diagram of control surface structural arrangement and forces. . . . 81

3.22 Outboard surface $C_{p}$ for a trailing edge flap deflection of $10^{\circ}$ over the last $10 \%$ of chord (output from XFoil) . . . . . . . . . . . . . . . . 81

3.23 Structural force discretisation. . . . . . . . . . . . . . . . . . . . 83

3.24 Resolution of moment and torque couples across a wing-sweep discontinuity. . 84

3.25 Assumed Boeing 737-200 configuration. . . . . . . . . . . . . . . . 86

3.26 Wing shear force, bending moment and torque diagrams - initial estimate (solid x-marked line) and final estimate (solid line) . . . . . . . . . . . . . 87

4.1 NACA $65_{4}-021$ incompressible surface velocity distribution $-C_{l}=0$. . .

4.2 Comparison of calculated suction distribution - NACA $65_{4}-021$ aerofoil, $C_{l}=0$ and $R e_{c}=20 \times 10^{6} \ldots \ldots \ldots \ldots \ldots \ldots \ldots \ldots \ldots \ldots \ldots \ldots \ldots$

4.3 Comparison of calculated momentum thickness distribution - NACA 654-021

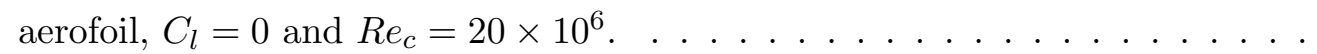

4.4 Section pressure (solid) and suction velocity distribution (dashed) for the upper surface of a NACA0028 aerofoil $-M_{\infty}=0.56, R e_{c}=40 \times 10^{6}$ and $C_{l}=0.15$.

4.5 Distribution of dynamic pressure loss through boundary layer (solid), and suction velocity (dashed) for the upper surface of a NACA0028 aerofoil $M_{\infty}=0.56, R e_{c}=40 \times 10^{6}$ and $C_{l}=0.15 \ldots \ldots \ldots \ldots$

4.6 Distribution of (area-distributed) power to overcome losses through boundary layer. . . . . . . . . . . . . . . . . . . . . . 100

4.7 Variation of $C_{Q}$ and $\widetilde{W}_{p}$ with $R e_{c}$ : NACA0028, $C_{l}=0.15$ and $M_{\infty}=0.56$. Both variables are scaled by $\sqrt{R e_{c}}$, so that a constant level implies proportionality to $R e_{c}^{-1 / 2} \ldots \ldots \ldots \ldots \ldots \ldots \ldots$

4.8 Dependence of distributed suction on section thickness for a range of symmetrical 4-digit NACA aerofoils at $C_{l}=0.15, M_{\infty}=0.5$ and $R e_{c}=40 \times 10^{6}$ : solid line $-10 \%$; dashed line $-20 \%$; and dashed-dotted line $-30 \%$; ' $x$ ' markers

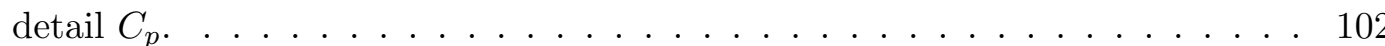

4.9 Variation of (a) $C_{Q}$ and (b) $\widetilde{W}_{p}$ with maximum thickness-to-chord ratio and section lift coefficient - symmetrical 4-digit NACA aerofoil series, $M_{\infty}=0.5$ and $R e_{c}=40 \times 10^{6}$. 
4.10 Variation of $C_{Q}$ (solid line) and $\widetilde{W}_{p}$ (dashed line) with free-stream Mach number and maximum thickness-to-chord ratio - symmetrical 4-digit NACA aerofoil series, $C_{l}=0.15$ and $R e_{c}=40 \times 10^{6} \ldots \ldots \ldots \ldots$. . . . . . 104

4.11 Suction hardware layout for an untapered wing of span $b$ and chord $c$. . . . 105

4.12 Schematic diagram of surface pressure variation over a single chamber width (downstream of the suction peak). . . . . . . . . . . . . . . 109

4.13 Chordwise distribution of differential pressure across the porous skin with the critical pressure superimposed. . . . . . . . . . . . . . . . . . . . 110

4.14 Variation of the total number of chambers with $\Delta \widetilde{P}_{s, a v}$ and $\tilde{r}_{c} \ldots \ldots \ldots 111$

4.15 Dependence of $C_{Q}$ on $\Delta \widetilde{P}_{s, a v}$ and $\tilde{r}_{c}$. The optimal value calculated by the algorithm of Sec. 4.1 .2 is $C_{Q}=2.24 \times 10^{-4} \ldots \ldots \ldots \ldots 111$

4.16 Variation of chordwise distribution of suction with $\tilde{r}_{c}$ and $\Delta \widetilde{P}_{s, a v} \ldots \ldots \ldots 112$

4.17 Variation of actual (solid lines) and approximate ('x' markers) $\widetilde{W}_{p}$ with $\Delta \widetilde{P}_{s, a v}$

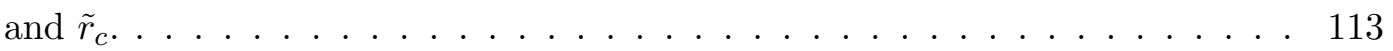

4.18 Notation for chamber flow. . . . . . . . . . . . . . . . . . . 114

4.19 Spanwise-chamber pressure drop dependence on $b / h$ and $w_{c} / h$ with $h=10 \mathrm{~cm} .115$

4.20 Schematic diagram of an alternative chamber-collector-duct arrangement. . . 116

4.21 Comparison of surface, chamber and collector static pressures $-\Delta \widetilde{P}_{s, a v}=0.15$

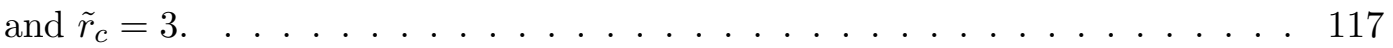

4.22 Variation of $\widetilde{W}_{p}$ with the number of collectors and $\tilde{r}_{c}$, with $\Delta \widetilde{P}_{s, a v}=0.15 \ldots \quad$. 118

5.1 Altitude against velocity with contours of Mach number and unit Reynolds number. . . . . . . . . . . . . . . . . . . . . . 122

5.2 Schematic diagram of a simplified laminar-flying-wing planform layout. . . . . 123

5.3 Wing sweep angle dependence on thickness-to-chord ratio and unit Reynolds

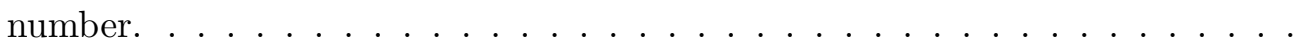

5.4 Zero-lift drag breakdown and its dependence on thickness-to-chord ratio and unit Reynolds number. . . . . . . . . . . . . . . . . . . . . . . . .

5.5 The dependence of cruise lift coefficient and lift-to-drag ratio on thickness-tochord ratio for: (a) a range of spans and a fixed $R e_{u}$ of $8 \times 10^{6} \mathrm{~m}^{-1}$ and, (b) a range of unit Reynolds numbers and a fixed span of $80 \mathrm{~m}$. . . . . . . . .

5.6 Cruise Mach number dependence on thickness-to-chord ratio, span and unit Reynolds number. (Top figure corresponds to a $R e_{u}=8 \times 10^{6} \mathrm{~m}^{-1}$, and the bottom to a span of 80 m.) . . . . . . . . . . . . . . . . . . 132 
5.7 The dependence of cruise altitude and cruise velocity on thickness-to-chord ratio for: (a) a range of spans and a fixed $R e_{u}$ of $8 \times 10^{6} \mathrm{~m}^{-1}$ and, (b) a range of unit Reynolds numbers and a fixed span of $80 \mathrm{~m} . \ldots \ldots$. . . . . .

5.8 Variation of aircraft wing loading with thickness-to-chord ratio, span and unit Reynolds number. (Top figure corresponds to $R e_{u}=8 \times 10^{6} \mathrm{~m}^{-1}$, and the bottom to a span of $80 \mathrm{~m}.) \ldots \ldots \ldots \ldots$

5.9 Variation of required maximum lift coefficient with thickness-to-chord ratio, span and unit Reynolds number. (Top figure corresponds to a $R e_{u}=8 \times 10^{6}$ $\mathrm{m}^{-1}$, and the bottom to a span of $80 \mathrm{~m}$.) . . . . . . . . .

5.10 Variation of aircraft allowable weight with thickness-to-chord ratio, span and unit Reynolds number. (Top figure corresponds to a $R e_{u}=8 \times 10^{6} \mathrm{~m}^{-1}$, and the bottom to a span of $80 \mathrm{~m}.) \ldots \ldots \ldots \ldots$

5.11 Variation of wing-weight fraction with thickness-to-chord ratio, span and unit Reynolds number. (Top figure corresponds to a $R e_{u}=8 \times 10^{6} \mathrm{~m}^{-1}$, and the bottom to a span of $80 \mathrm{~m}.) \ldots \ldots \ldots \ldots$

6.1 Variation of cabin half-width with roll-mode time constant for a range of vertical accelerations.

6.2 Schematic diagram of aircraft planform geometry: (a) $T_{R}=0.1$ and $H_{w}=5$ $\mathrm{m}$ and (b) $T_{R}=1$ and $H_{w}=2 \mathrm{~m} \ldots \ldots \ldots \ldots$

6.3 Variation in longitudinal NP position over a range of outboard wing taper

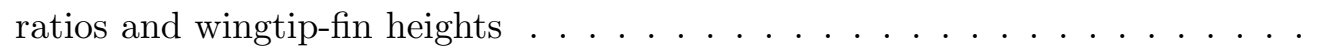

6.4 Variation in lateral NP position over a range of outboard wing taper ratios and wingtip-fin heights . . . . . . . . . . . . . .

6.5 Change in (a) yawing moment coefficient and (b) side force with sideslip angle over a range of outboard wing taper ratios and wingtip-fin heights. . . . . . . 153

6.6 Aerofoil section generator. . . . . . . . . . . . . . . . . . . . . . . . . 154

6.7 Schematic diagram of the multi-bubble cabin embedded within an aerofoil section - vertical spar locations are represented by the dash-dot lines, whilst the minimum spacings between the cabin and wing surface are indicated by the 'x' markers. . . . . . . . . . . . . . . . . . . . . . . . . . . .

6.8 Three dimensional view of wing half-span, spanwise variations of aerofoil section geometry, multi-bubble cabin arrangement, and $C_{p}$ distribution at the cruise (with suction) condition: centrebody $M_{\perp}=0.63$, outboard $M_{\perp}=0.61$, wingtip-fin $M_{\perp}=0.67$; see Fig. 6.9 for $C_{l_{\perp}}$ values. $\ldots \ldots \ldots \ldots$ 
6.9 Spanwise variation in section lift coefficient in the free-stream and wing normal directions $-C_{L}=0.14$ and $M_{\infty}=0.67 \ldots \ldots \ldots \ldots \ldots$

6.10 Variation in viscous drag coefficient along the wing half-span $-R e_{u}=8 \times 10^{6}$ $\mathrm{m}^{-1} \ldots \ldots \ldots \ldots \ldots \ldots \ldots$

6.11 Force diagram at takeoff rotation. . . . . . . . . . . . . . . . . . 161

6.12 Spanwise lift distribution corresponding to a clockwise roll at take off with: $\delta_{a}=+/-12.2^{\circ}$ and $\delta_{E}=-2.5^{\circ}: M_{\infty}=0.21$ and $C_{L}=0.61 \ldots \ldots 3$

6.13 Typical inboard suction-system architecture. . . . . . . . . . . . . . . 165

6.14 Distribution of inboard chamber and surface (non-dimensional) pressures across

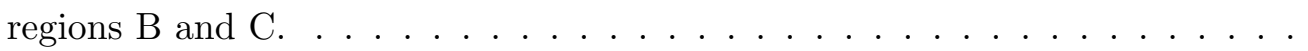

6.15 Critical (non-dimensional) differential pressures across porous skin along the centrebody region. . . . . . . . . . . . . . . . . . 168

6.16 Outboard (non-dimensional) chamber pressure drop. . . . . . . . . . . . . . 169

6.17 Variation in overall suction coefficient and pump drag coefficient along the wing half-span. $C_{Q}$ is $3.10 \times 10^{-4}$ and $\widetilde{W}_{p}$ is $6.27 \times 10^{-4}-R e_{u}=8 \times 10^{6}$ $\mathrm{m}^{-1}, \eta_{p}=0.85 \ldots \ldots \ldots \ldots \ldots \ldots \ldots \ldots$

6.18 Two engine turbofan HPC operating map, detailing the TOC design point (white circle), and takeoff off-design point for increasing engine mass flow rate (yellow squares). The non-dimensional spool speed $(\mathrm{N})$ is represented by the solid black contours, and the compressor efficiency by the red contours. . . .

6.19 Net thrust, HPC exit temperature and burner exit temperature variation with engine mass flow rate at TO conditions for a two-engine turbofan designed for

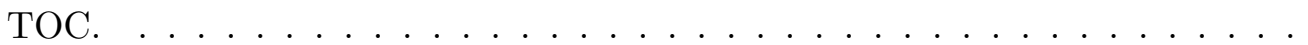

6.20 Two engine turbofan HPC operating map, detailing the TO design point (white circle), and various off-design points (yellow squares). The non-dimensional spool speed $(\mathrm{N})$ is represented by the solid black contours, and the compressor efficiency by the red contours. The design point has been rescaled with $\beta=0.3$

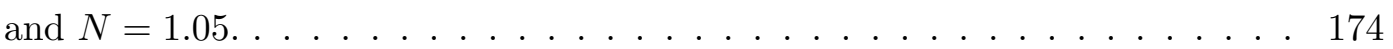

6.21 Three engine turbofan HPC operating map, detailing the cruise-without-suction design point (white circle), and various off-design points (yellow squares). The non-dimensional spool speed $(\mathrm{N})$ is represented by the solid black contours, and the compressor efficiency by the red contours. The design point has been rescaled with $\beta=0.3$ and $N=1.05 . \ldots \ldots \ldots \ldots \ldots$

6.22 Propeller map for a three-engine turboprop with TO as the design point. (Solid black lines represent contours of propeller efficiency.) . . . . . . . . . . . 176 
6.23 Three engine turboprop HPC operating map, detailing the TO design point (white circle), and various off-design points (yellow squares). The non-dimensional spool speed $(\mathrm{N})$ is represented by the solid black contours, and the compressor efficiency by the red contours. The design point has been rescaled with $\beta=0.2$

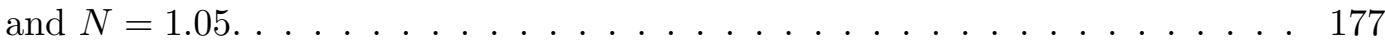

6.24 HPC operating map detailing the takeoff design point, various off-design points, non-dimensional spool speed $(\mathrm{N})$ contours (solid black), and efficiency contours (red). The design point has been rescaled with $\beta=0.2$ and $N=1.05 . \ldots 179$

6.25 Variation of cruise (with suction) engine $s f c$ with thrust. . . . . . . . . . . 182

6.26 Schematic diagram of LFW fuselage structural arrangement. . . . . . . . . . 183

6.27 Wing structural geometry. . . . . . . . . . . . . . . . . . . . . 184

6.28 The distribution of weights and point loads over the planform is shown in a), whilst in b) and c) the root cantilever model is illustrated for the in-flight and on-ground load cases. . . . . . . . . . . . . . . . . . . . . . 186

6.29 Shear force, bending moment and torque diagrams for critical load cases initial estimate for in-flight (solid line), final estimate including self weight for in-flight (dashed line) and final estimate for ground (solid-star line). . . . . . 187

6.30 Tricycle landing gear geometric arrangement. . . . . . . . . . . . . . . . . . 190

6.31 Tipback geometry. . . . . . . . . . . . . . . . . . . . . . . . . 191

6.32 Overturn geometry. . . . . . . . . . . . . . . . . . . . . . 192

6.33 Aft tail positioning (from Raymer (1999)) . . . . . . . . . . . . . 195

6.34 Competitor aircraft configuration. . . . . . . . . . . . . . . . . 197

6.35 Spanwise loading distributions for $C_{L}=0.48$ and $M_{\infty}=0.67-C_{l}$ (dashed)

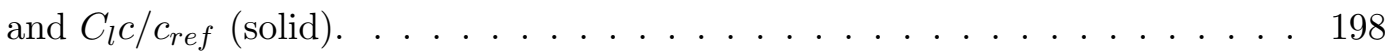

6.36 Surface $C_{p}$ distribution for an RAE2822 aerfoil, with the critical $C_{p}$ superposed

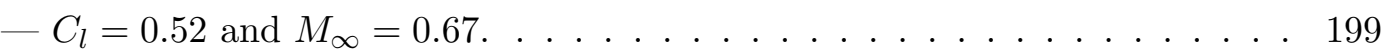

6.37 Comparison of LFW and competitor aircraft MTOW breakdowns. . . . . . . 204 


\section{List of TABLES}

2.1 Aerofoil drag comparisons on the F-94A upper surface, all for $R e_{c} \approx 30 \times 10^{6}$ $($ from Green $(2006)) \ldots \ldots \ldots \ldots 12 \ldots \ldots \ldots$

2.2 HP117 aircraft design specification (from Lee (1961)) . . . . . . . . . . . . 41

3.1 Summary of two-spool turboprop iteration variables and errors. . . . . . . . . 64

3.2 Summary of two-spool, unmixed flow, turbofan iteration variables and errors. $\quad 65$

3.3 Estimated wing structural weight breakdown for the B737-200. . . . . . . . 88

3.4 Estimated fuselage structural weight breakdown for the B737-200. . . . . . . 88

3.5 Structural weight breakdown for the B737-200 . . . . . . . . . . . . . 89

3.6 Operating empty weight breakdown for the B737-200 . . . . . . . . . . . 90

5.1 Laminar-flying-wing aircraft design specification. . . . . . . . . . . . . . . 140

6.1 Static stability - C.G. locations (see Sec. 6.1.5.5): $11.38 \mathrm{~m}$ (start of cruise and climb out) and $11.44 \mathrm{~m}$ (end of cruise) . . . . . . . . . . . 152

6.2 LFW section geometry variables specification. . . . . . . . . . . . . . . 156

6.3 LFW lift and drag coefficient breakdown. . . . . . . . . . . . . . . . 163

6.4 LFW aerodynamic performance parameters for climb-out and takeoff with one engine inoperative, and cruise, with C.G. at 11.38 m. . . . . . . . . . . . . 164

6.5 Chamber specifications for the inboard section. . . . . . . . . . . . . 166

6.6 Chamber specifications for the outboard section. . . . . . . . . . . . . 168

6.7 Thrust and suction power requirements (per engine) at various flight conditions for two/three engines. . . . . . . . . . . . . . . . . . . 171

6.8 Thrust and suction power requirements (per engine) at various flight conditions.179

6.9 LFW engine performance. . . . . . . . . . . . . . . . . . . . . . 181

$6.10 \mathrm{LFW}$ structural weight breakdown . . . . . . . . . . . . . . . . . 189

6.11 LFW aircraft weight buildup . . . . . . . . . . . . . . . . . . . . . 189

6.12 LFW mission-fuel weight buildup . . . . . . . . . . . . . . . . . . . 192

6.13 Competitor aircraft geometric parameters. . . . . . . . . . . . . . 196 
6.14 Competitor aircraft cruise lift and drag coefficient breakdown. . . . . . . . . 200

6.15 Competitor aircraft engine design variables. . . . . . . . . . . . . . . 200

6.16 Competitor aircraft engine performance summary. . . . . . . . . . . . . 201

6.17 Competitor aircraft wing structural weight buildup. . . . . . . . . . . . . 202

6.18 Competitor aircraft fuselage structural weight breakdown. . . . . . . . . . . 202

6.19 Competitor aircraft structural weight breakdown. . . . . . . . . . . . . 203

6.20 Competitor fuel weight breakdown. . . . . . . . . . . . . . . . . . 203

6.21 Competitor aircraft weight buildup. . . . . . . . . . . . . . . . . 204 


\section{Chapter 0}

\section{NomenClature}

\begin{tabular}{|c|c|c|c|}
\hline$A_{R}$ & $=$ aspect ratio & $D$ & $=$ diameter \\
\hline$b$ & $=\operatorname{span}$ & $e$ & $=$ Oswald efficiency \\
\hline$c$ & $=$ chord & $f$ & $=$ friction factor \\
\hline$d$ & $=$ suction hole diameter & $g$ & $=9.81 \mathrm{~m} / \mathrm{s}^{2}$, gravitational \\
\hline$C_{d}$ & $=$ dissipation coefficient & & acceleration \\
\hline$C_{D}$ & $=$ drag coefficient & $h$ & $=$ chamber height \\
\hline$C_{f}$ & $=$ wall skin friction coefficient & $H_{w}$ & $=$ wingtip-fin height \\
\hline \multirow[t]{2}{*}{$C_{l}$} & $=$ rolling-moment coefficient & $H_{12}$ & $=\delta_{1} / \delta_{2}$, shape factor \\
\hline & and section lift coefficient & $H_{32}$ & $=\delta_{3} / \delta_{2}$, energy shape factor \\
\hline$C_{L}$ & $=$ lift coefficient & $k$ & $=\widetilde{P}_{\infty}-\widetilde{P}_{c}$, non-dimensional \\
\hline$C_{m}$ & $=$ pitching-moment coefficient & & pressure difference constant \\
\hline \multirow[t]{2}{*}{$C_{M 0}$} & $=$ zero-lift pitching-moment & $K_{s}$ & $=$ skin resistance \\
\hline & coefficient & $\dot{m}$ & $=$ mass flow rate per unit width \\
\hline$C_{n}$ & $=$ yawing-moment coefficient & & along spanwise chamber \\
\hline \multirow[t]{2}{*}{$c_{p}$} & $=$ specific heat capacity of air & $\dot{m}_{f}$ & $=$ fuel mass flow rate \\
\hline & at constant pressure & $\dot{m}_{s}$ & $=$ area-distributed suction mass \\
\hline \multirow[t]{2}{*}{$C_{p}$} & $=\frac{P_{e}-P_{\infty}}{1}$, surface pressure & & flow rate \\
\hline & coefficient & $M$ & $=$ Mach number \\
\hline \multirow[t]{2}{*}{$C_{Q}$} & $=\oint \frac{V_{0}}{U_{\infty}} d(s / c)$, suction flow & $N$ & $=$ rotational speed \\
\hline & coefficient & $p$ & $=$ roll rate \\
\hline \multirow[t]{2}{*}{$D^{*}$} & $=$ dissipation function for a & $q \sim$ & $=$ pitch rate \\
\hline & laminar boundary layer & $P, \widetilde{P}$ & $=$ pressure $; \frac{P}{\frac{1}{2} \rho_{\infty} U_{\infty}^{2}}$, \\
\hline \multirow[t]{2}{*}{$D_{e q}$} & $=2 h \frac{w_{c} / h}{1+w_{c} / h}$, equivalent & & non-dimensional pressure \\
\hline & diameter for rectangular conduits & $r$ & $=$ yaw rate \\
\hline
\end{tabular}




\begin{tabular}{|c|c|c|c|}
\hline \multirow[t]{4}{*}{$\tilde{r}_{c}$} & $=\frac{\Delta \widetilde{P}_{s, \max }}{\Delta \widetilde{P}_{s \min }}$, ratio & $Y$ & $=$ side force \\
\hline & $\begin{array}{l}\Delta P_{s, \min } \\
\text { of non-dimensional pressure }\end{array}$ & $z, \tilde{z}$ & $=$ spanwise distance, $z / b$ \\
\hline & difference across skin over a & $Z$ & $=$ normal force \\
\hline & single chamber width & $C_{n_{\beta}}$ & $=d C_{n} / d \beta$ \\
\hline \multirow[t]{2}{*}{$R e_{D_{e q}}$} & $=\frac{2 \dot{m}}{\mu} \frac{w_{c} / h}{1+w_{c} / h}$, spanwise-chamber & $C_{Y_{\beta}}$ & $=d C_{Y} / d \beta$ \\
\hline & $\begin{array}{l}\mu 1+w_{c} / h \\
\text { Reynolds number }\end{array}$ & $C_{l_{\beta}}$ & $=d C_{l} / d \beta$ \\
\hline \multirow[t]{2}{*}{$R e_{h}$} & $=V_{h} d / \nu$, suction hole & $C_{l_{r}}$ & $=\frac{\partial C_{l}}{\partial\left(r b / 2 U_{\infty}\right)}$ \\
\hline & Reynolds number & $C_{n_{r}}$ & $=\frac{\partial C_{n}}{\partial\left(r b / 2 U_{\infty}\right)}$ \\
\hline \multirow[t]{2}{*}{$R e_{\delta_{2}}$} & $=u_{e} \delta_{2} / \nu$, momentum & $C_{m_{q}}$ & \\
\hline & Reynolds number & Symbols & \\
\hline$s$ & $=$ intrinsic distance along aerofoil & & \\
\hline$S$ & $=$ area & $\alpha$ & $=$ angle of attack \\
\hline \multirow[t]{2}{*}{$t$} & $=$ maximum aerofoil thickness, & $\beta$ & $=$ sideslip angle \\
\hline & and time & $\delta$ & $=$ control surface deflection; boundary \\
\hline$t_{s}$ & $=$ porous skin thickness & & layer thickness \\
\hline$T$ & $=$ temperature & $\delta_{1}, \delta_{2}, \delta_{3}$ & $=$ Boundary layer displacement, \\
\hline$T_{R}$ & $=$ outboard wing taper ratio & & momentum and kinetic energy \\
\hline$T_{02}$ & $=$ compressor exit temperature & & thickness \\
\hline$T_{04}$ & $=$ burner exit temperature & $\Delta P, \Delta \widetilde{P}$ & $=$ Pressure difference $; \frac{\Delta P}{\frac{1}{2} \rho_{\infty} U_{\infty}^{2}}$, \\
\hline \multirow[t]{2}{*}{$u$} & $=$ streamwise velocity inside & & non-dimensional pressure difference \\
\hline & boundary layer & $\epsilon^{*}$ & $=$ skin-friction function for a \\
\hline \multirow[t]{2}{*}{$u_{e}$} & $=$ local velocity outside boundary & & laminar boundary layer \\
\hline & layer & $\Lambda$ & $=$ wing sweep angle \\
\hline$U$ & $=$ velocity & $\nu$ & $=$ kinematic viscosity \\
\hline \multirow[t]{2}{*}{$V_{0}$} & $=$ area-distributed suction & $\phi$ & $=$ bank angle \\
\hline & velocity & $\rho$ & $=$ density \\
\hline$V_{h}$ & $=$ hole suction velocity & $\sigma$ & $=$ stress \\
\hline$w$ & $=$ normal velocity & $\tau_{R}$ & $=$ roll-subsidence time constant \\
\hline$w_{c}$ & $=$ chamber width & & \\
\hline$w_{L}$ & $=$ wing loading & Subscripts & \\
\hline \multirow[t]{2}{*}{$\tilde{w}_{p}, \widetilde{W}_{p}$} & $=$ area-distributed, and total, & $a$ & $=$ aileron \\
\hline & power coefficient & $a v$ & $=$ average \\
\hline$W$ & $=$ weight & $c$ & $=$ chord, and chamber \\
\hline$x$ & $=$ chordwise distance & $C$ & $=$ compressor \\
\hline & $=$ lateral coordinate & $d$ & $=$ duct \\
\hline
\end{tabular}




\begin{tabular}{|c|c|c|c|}
\hline$e$ & $=$ engine, and boundary-layer edge & 0 & $=$ zero lift, and stagnation \\
\hline$e x$ & $=$ pump exit & & quantity \\
\hline$E$ & $=$ elevator & $\infty$ & $=$ free stream \\
\hline$h$ & $=$ hole & $\perp$ & $=$ perpendicular \\
\hline$i$ & $=$ induced & & \\
\hline in & $=$ pump inlet & \multicolumn{2}{|c|}{ Abbreviations } \\
\hline$j$ & $=$ jet & APU & $=$ auxiliary power unit \\
\hline $\max$ & $=$ maximum & BPR & $=$ bypass ratio \\
\hline$P R$ & $=$ propeller & $\mathrm{CG}$ & $=$ centre of gravity \\
\hline$r$ & $=$ rudder & $\mathrm{CF}$ & $=$ crossflow \\
\hline ref & $=$ reference & FW & $=$ flying wing \\
\hline$s$ & $=$ skin & $\mathrm{HPC}$ & $=$ high pressure compressor \\
\hline$s p$ & $=$ suction pump & LCV & $=$ low calorific value \\
\hline te & $=$ trailing edge & LFC & $=$ laminar flow control \\
\hline$t h$ & $=$ thermal & LFW & $=$ laminar flying wing \\
\hline$u$ & $=$ unit & MTOW & $=$ maximum takeoff weight \\
\hline$u c$ & $=$ undercarriage & OEW & $=$ operating empty weight \\
\hline$U T S$ & $=$ ultimate tensile strength & PPR & $=$ pump pressure ratio \\
\hline$v$ & $=$ viscous & sfc & $=$ specific fuel consumption \\
\hline$w$ & $=$ wall & $\mathrm{T}-\mathrm{S}$ & $=$ Tollmien-Schlichting \\
\hline wet & $=$ wetted & & \\
\hline
\end{tabular}


CHAPTER 0. NOMENCLATURE

xxxii 


\section{Chapter 1}

\section{INTRODUCTION}

With the continued growth in air travel, focus is being increasingly drawn to the environmental impact of engine combustion products. The technology sub-group of Greener by Design, led by Green (2002), considers that, amongst the various environmental issues, the reduction of $\mathrm{CO}_{2}$ emissions is likely to emerge as the most important. For kerosene-fuelled aircraft, this translates directly to a requirement to reduce fuel burn, i.e. the amount of fuel consumed per unit passenger-range measured over a whole journey.

Over the last five decades aircraft have changed little in their appearance and operating conditions. They are characterised by a high-aspect-ratio wing joined to a slender fuselage, with a tailplane located at the rear and pod-mounted engines, as shown in Fig. 1.1. Furthermore, they operate with turbulent boundary layers at cruise altitudes between 30,000 40,000 ft in a high-subsonic Mach number range of $0.8-0.85$.

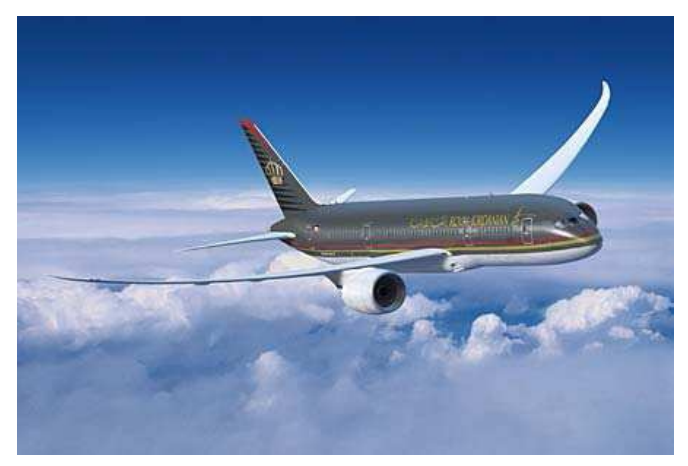

Figure 1.1: Conventional aircraft layout — the Boeing 787 Dreamliner.

Despite significant improvements in efficiency since its introduction, Fig. 1.2 shows that the performance of this configuration appears to be approaching an asymptote. The trends in engine fuel consumption suggests that these improvements have largely been due to advance- 
ments in engine technology. Furthermore, Denning et al. (1997) comment that improved materials and further drag reductions for swept-wing turbulent aircraft are likely to have limited benefit. For this reason, researchers, such as Green (2002) and Greitzer et al. (2010), have considered a number of alternatives.

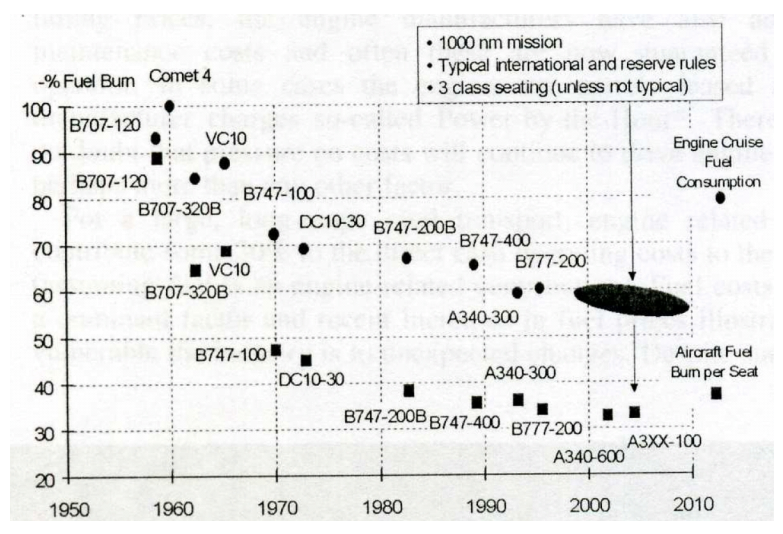

Figure 1.2: Engine fuel consumption and fuel burn per seat over a $1000 \mathrm{~nm}$ mission (from Birch (2000)).

One such alternative solution, still based on the tube and wing concept, has been proposed by Greitzer et al. (2010) with the D8 series aircraft. The arrangement, shown in Fig. 1.3, consists of a double-bubble fuselage with a lifting nose, embedded aft engines, pi tail arrangement, and reduced Mach number operations. The overall estimated reduction in fuel burn, with the B737-800 as a baseline and assuming current technology levels, is given at $49 \%$. (This improvement is projected to increase to $71 \%$ on incorporation of advanced technologies such as: ultra high bypass ratio engines, advanced structural materials and natural laminar flow.) The benefits from flying slower are an aircraft with: an increased aspect ratio, permitted by unsweeping the wing; a reduced empty weight; and, a lower minimum static margin, which reduces trim drag. Due to these changes alone, the fuel burn reduction is estimated at $8 \%$.

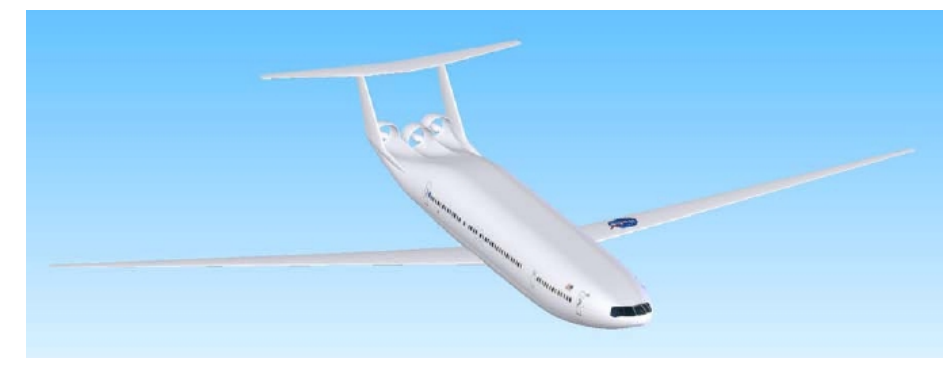

Figure 1.3: D8 aircraft series (from Greitzer et al. (2010)). 
Blended wing-body (BWB) aircraft with turbulent boundary layers have received much attention in recent years. The concept is shown in Fig. 1.4. It consists of swept outer wing regions with a 'wing-cum-fuselage' centrebody. The configuration benefits from the absence of fuselage and tail drag, at the cost of greater difficulty in achieving satisfactory longitudinal stability and control characteristics (Nickel and Wohlfart (1994)). Furthermore, the study of Bolsunovsky et al. (2001) has shown that, with the distribution of payload and low aspect ratio configuration, takeoff weight is likely to be lower than an equivalent swept wing aircraft. Based on the concept of Bolsunovsky et al. (2001), Green (2002) estimates a $23 \%$ reduction in fuel burn on a baseline swept-wing aircraft design for the same mission specification.

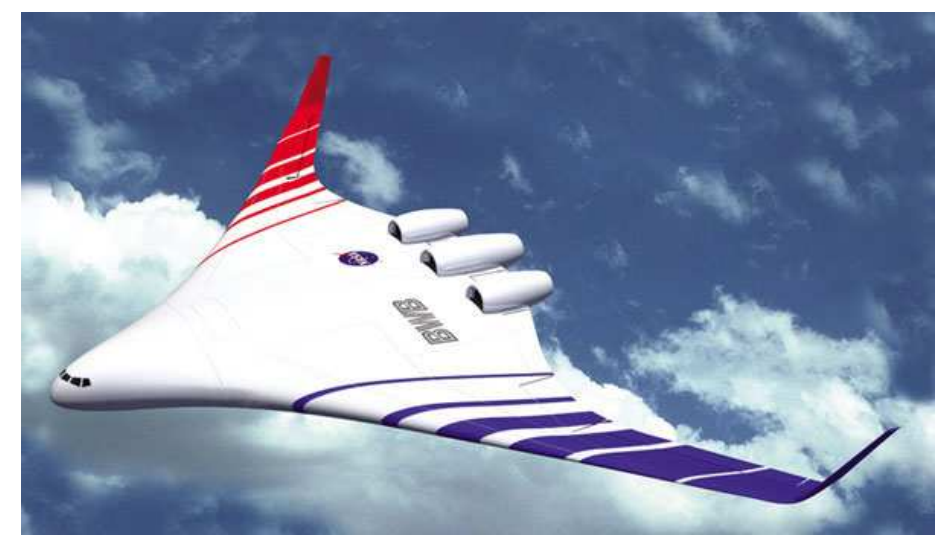

Figure 1.4: Boeing X-48B blended wing body.

Assuming present day technology, Green (2002) identifies the 'laminar flying wing' (LFW) as having the greatest potential for fuel-burn reduction. The concept consists of a flyingwing (FW) arrangement, which utilises distributed suction through porous wing surfaces as a means of laminar flow control (LFC). The flying wing has had proponents since the early days of powered flight, and has been physically realised in a number of designs over the intervening years; one such example is the Northrop YRB-49 bomber shown in Fig. 1.5, developed in the 1950's. The configuration differs from that of a BWB as the entire FW consists of a single lifting surface, whilst the BWB's centrebody and wing are still distinguishable from one another with their markedly different shapes.

The flying wing has traditionally been viewed as unsuitable for passenger aircraft, because of accommodation issues. Nonetheless, Martinez-Val et al. (2006) have argued that an 80mspan flying wing with a passenger capacity of 300 and a fuel burn of $19.8 \mathrm{~g} / \mathrm{pax} . \mathrm{km}$ is a practical possibility. Such a vehicle would be approximately $16 \%$ more efficient than a Boeing 777 aircraft, which has a fuel burn of $23.5 \mathrm{~g} /$ pax.km (Martinez-Val et al. (2006)). Torenbeek (1991) has also considered the potential of the flying wing for passenger transport, as one of 


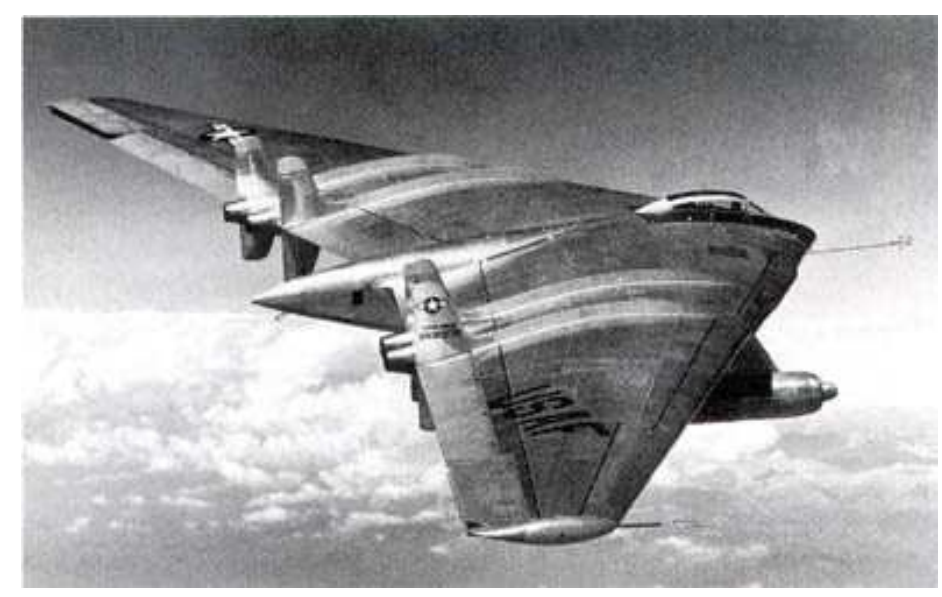

Figure 1.5: Northrop YRB-49 bomber.

a range of wing-body configurations, all having the same useful volume. His conclusions are less optimistic (because the increased wetted area of a flying wing offsets the drag benefit of eliminating the fuselage), and suggest that a pure flying wing does not necessarily represent the optimum allocation of usable volume between a body and a wing.

The flying-wing configuration is, however, unambiguously superior once LFC is envisaged. Laminar flow control reduces skin-friction drag by suppressing boundary-layer transition, usually via surface suction. On a conventional aircraft, Green (2006) considers the application of LFC to the wing, fin, tail and nacelle surfaces (arguing that it cannot practically be applied to the fuselage, whilst Denning et al. (2003) add that problems also arise towards the rear of wings with flaps and spoilers), and estimates a fuel burn reduction of just $16 \%$ on a baseline turbulent aircraft for the same mission. However, on a flying wing it can lead to a very significant drag reduction. The LFW proposed by Greener by Design is a flying-wing aircraft with LFC over (almost) all its surface. On the basis of the Breguet range equation, and estimates for its component parameters (assuming 2001 technology levels), Green (2006) suggests that a turbofan powered LFW with the same payload ( $86 \mathrm{mt})$ and range $(12,500 \mathrm{~km})$ has a fuel burn that is around $50 \%$ below that of a baseline-turbulent aircraft. Alternatively, Green (2002) considers an unducted-fan configuration, at the same technology level, and this figure is expected to increase to around $60 \%$. Finally, at 2050 technology levels, a fuel-burn reduction of $70 \%$ is anticipated (Green (2002)).

This thesis begins in Chap. 2 with a review of the open literature on laminar flow control, flying wing aircraft, and studies which combine the two technologies. Chapter 3 gives an overview of the design methodology and numerical design tools used in the conceptual design evaluation. Chapter 4 then provides insight into the parameters affecting practical LFC 
suction power requirements for a commercial LFW transport aircraft. In Chap. 5 a viable basic aircraft design specification is given. Then, in Chap. 6, the detailed design of a LFW and a baseline, conventional, aircraft is undertaken. The thesis culminates with some conclusions and suggestions for future work in Chap. 7. 


\section{CHAPTER 2}

\section{LITERATURE REVIEW}

In this chapter, the open literature is surveyed. First, the well researched area of laminar flow control is discussed. Then the flying wing configuration is explored. Finally, the integration of these two technologies is examined.

\subsection{Laminar Flow Control}

The boundary layer was first discovered by Prandtl in 1904 (Anderson (1997)). It is a thin layer of fluid close to the surface of a body, over which the velocity reduces from its freestream value at the edge of the boundary layer to zero at the wall. The boundary layer can be categorised as either: laminar, where the flow is in smooth streamlines parallel to the surface; or turbulent, where the flow consists of irregular fluctions in velocity superposed on the mean flow, and roughly parallel to the surface (Young (1989)). The process of change between these two states is known as transition. The velocity fluctuations within a turbulent boundary layer provide a powerful mechanism for mixing, and lead to a higher rate of strain, and hence wall shear stress, relative to laminar boundary layers. Consequently, laminar skin friction can be as much as $90 \%$ less than turbulent skin friction at the same Reynolds number (Joslin (1998a)). The concept of applying surface suction to stabilise a laminar boundary layer and limit its growth, thereby reducing drag, is not new. Much of the work on full-chord LFC with permeable surfaces took place in the 1940s through the mid-1960s, pioneered by Lachmann in the UK and Pfenninger in the USA. More contemporary laminar flow control studies have mainly been concerned with hybrid laminar flow control (HLFC) systems, which integrate the ideas of natural laminar flow with surface suction (Denning et al. (1997)).

This section begins with an exploration of the basic concept of boundary layer stability and its control through the application of suction. Then the design of LFC suction systems is discussed. 


\subsubsection{Profile Drag Reduction}

Two dimensional boundary layers are characterised by their velocity profile, as shown in Fig. 2.1. In the absence of flow control, the shape of the velocity profile depends solely on the streamwise pressure gradient. A favourable pressure gradient leads to a fuller velocity profile with a negative curvature $\left(\partial^{2} u / \partial y^{2}\right)$. In contrast, an adverse pressure gradient leads to a less full profile with a region of positive curvature, and the consequent existence of an inflection point.

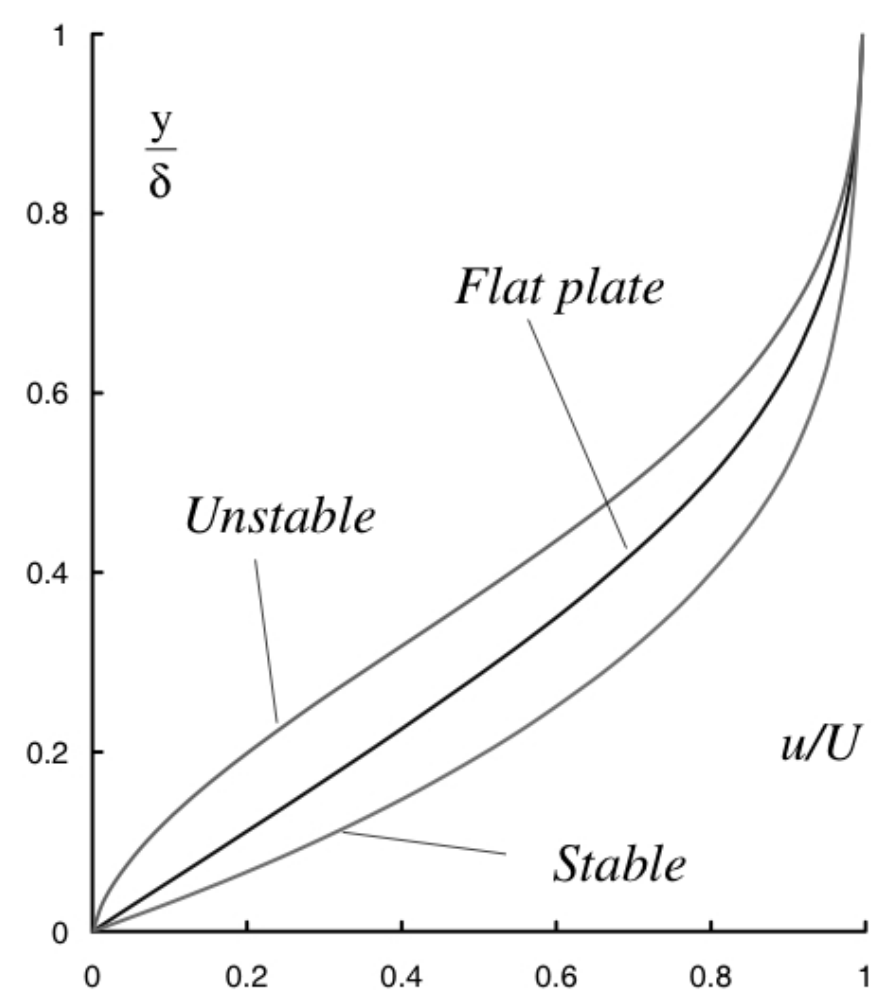

Figure 2.1: Stable and unstable velocity profiles in a flat-plate laminar boundary layer — flat plate corresponds to zero-pressure gradient. (Taken from Green (2006).)

The realm of stability theory is beyond the scope of this thesis; however, Green (2006) notes that for flows in which the velocity profile has an inflection point, transition occurs at much lower Reynolds numbers compared to the zero pressure-gradient case. In contrast, the opposite applies for a more convex velocity profile.

The change of boundary layer thickness and velocity profile due to suction is detailed schematically by Lachmann (1955) in Fig. 2.2. The effect of suction is two-fold: 1) the boundary layer profile is made more convex, and 2) the boundary layer thickness (and hence 


\subsection{LAMINAR FLOW CONTROL}

local Reynolds number) is reduced. This combination leads to a more stable boundary layer.

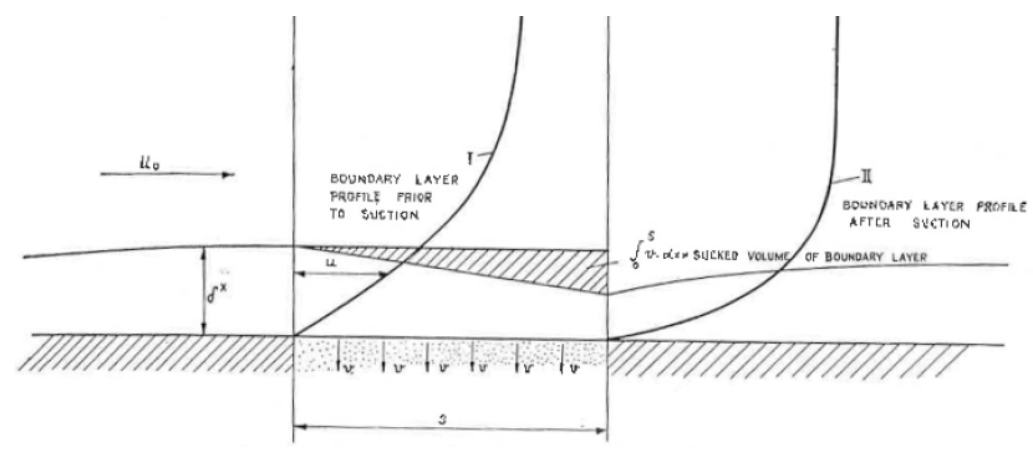

Figure 2.2: Boundary layer development with suction (from Lachmann (1955)).

These observations can be understood through consideration of the incompressible boundary layer momentum equation with surface suction at the wall, which Green (2006) gives to be

$$
\mu \frac{\partial^{2} u}{\partial y^{2}}=\frac{d p}{d x}-\rho V_{0}\left(\frac{\partial u}{\partial y}\right)_{w} .
$$

This expression shows that, as expected, flow acceleration and suction through the wall both promote a convex velocity profile.

The boundary layer is characterised by two integral parameters: the displacement and momentum thickness. Displacement thickness $\left(\delta_{1}\right)$ is a measure of the displacement of the free stream away from the surface due to boundary-layer growth, whilst momentum thickness $\left(\delta_{2}\right)$ is a measure of the momentum deficit. These definitions are retained downstream of the aircraft and, for two-dimensional shock-free flow, $\delta_{2}$ far downstream is directly proportional to the drag of the body.

$$
\begin{aligned}
\delta_{1} & =\int_{0}^{\infty}\left(1-\frac{u}{u_{e}}\right) d y \\
\delta_{2} & =\int_{0}^{\infty}\left(\frac{u}{u_{e}}\left(1-\frac{u}{u_{e}}\right)\right) d y
\end{aligned}
$$

On integration of Eq. 2.1, combined with the continuity equation and Eqs. 2.2 and 2.3, von Kármán derived the momentum integral equation

$$
\frac{d \delta_{2}}{d x}+\left(2+H_{12}\right) \frac{1}{u_{e}} \frac{d u_{e}}{d x} \delta_{2}=C_{f}-\frac{V_{0}}{u_{e}},
$$




\section{CHAPTER 2. LITERATURE REVIEW}

where the shape factor

$$
H_{12}=\frac{\delta_{1}}{\delta_{2}} .
$$

Equation 2.4 can be re-written in a form that shows the influence of suction on the rate of boundary layer growth

$$
\rho \frac{d}{d x}\left(u_{e}^{2} \delta_{2}\right)=\delta_{1} \frac{d P}{d x}+\tau_{w}-\frac{V_{o}}{u_{e}} .
$$

Green (2006) highlights that the term on the left is the rate of growth of momentum deficit in the boundary layer, whilst the first and second terms on the right are the contributions to local pressure and friction drag. He explains that when $d P / d x$ is large in comparison to the skin friction term, the increase in momentum deficit, and hence drag, is proportional to the local thickness.

Drawing largely from a paper by Edwards (1961), Lee (1961) attempts to clarify some common misconceptions of how a reduction in profile drag through surface suction is actually manifested. He explains that, as expected of a laminar boundary layer, the pressure and skin friction drags are low. However, there is a limit to this: excessive suction will produce a boundary layer that is extremely thin, with an associated skin friction coefficient that can approach, or even exceed, that of a thick turbulent boundary layer. (Note, though, that a turbulent boundary layer would result in a larger wake with a larger amount of unrecovered energy left behind in the atmosphere.) Lee (1961) concludes that the application of suction should be such that the boundary layer remains on the verge of transition.

Lachmann (1955) highlights that the need to re-energise the sucked air was investigated by Pfenninger. Early experiments demonstrated an additional $40 \%$ reduction in effective drag if the (stagnant) sucked flow is accelerated to free-stream velocity. Therefore, the sucked low momentum fluid, which would otherwise need to be cancelled by an equal and opposite momentum increase in the propulsion wake, does not appear as a deficit in the wake, as illustrated in Fig. 2.3. To summarise, the actual drag reduction effects are: 1) a reduction of wake drag to a practically insignificant magnitude, and 2) a recovery with high mechanical efficiency of the kinetic energy of the air which has been sucked from the lower, almost stagnant, strata of the boundary layer.

In contrast to natural laminar flow, Lachmann (1955) observes that the critical parameter for laminar flow with distributed suction is Reynolds number per unit chord. Denning et al. (1997) interpret this result as there being no upper limit to wing chord size because the boundary layer is continually 'regenerating itself'.

The maximum possible unit Reynolds number depends on the surface finish and at mospheric turbulence levels (Lachmann (1955)); these effects become less significant with 


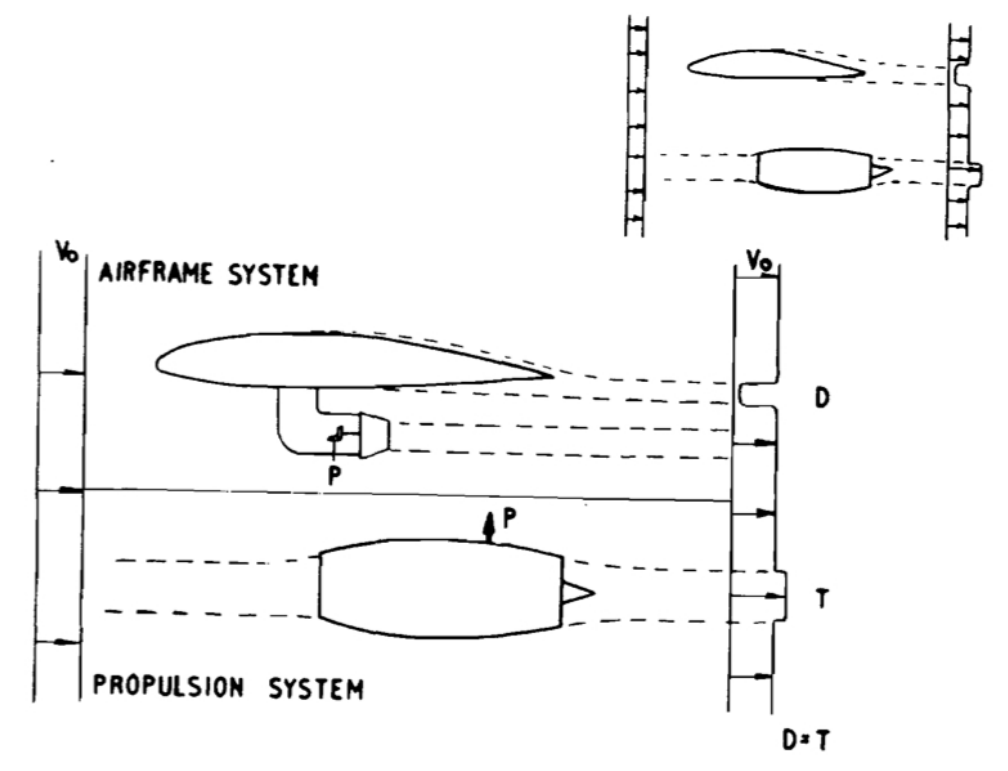

Figure 2.3: Wake profile for an integrated propulsion and suction system (from Lee (1961)).

increasing cruise altitude as a result of the increase in kinematic viscosity (Green (2006)). The F94-A aeroplane maintained full-chord laminar flow for unit Reynolds numbers of over $13 \times 10^{6} \mathrm{~m}^{-1}$; therefore, based on the state-of-the-art at the time, Lachmann (1955) proposes that unit Reynolds numbers of up to $16 \times 10^{6} \mathrm{~m}^{-1}$ may realistically achievable.

With the growth rate of a boundary layer with suction independent of chord length, the boundary layer thickness relative to the wing chord is reduced. This result implies a reduction in drag coefficient with $R e_{c}$. This is shown in Fig. 2.4. Furthermore, at high Reynolds numbers, e.g. $100 \times 10^{6}$, complete laminar flow aerofoils produce drag levels around one tenth of turbulent values.

There is a drawback with suction, however. Power is needed to energise the suction system, in order to develop a sufficient pressure ratio to maintain the desired flow rate. This is commonly referred to as an equivalent 'pump drag'.

Green (2006) provides illustrative profile and pump drag coefficients taken from the upper surface suction glove, flight-tested by Northrop, on the F-94A; these are repeated in Tab. 2.1. Green (2006) comments that the fully laminar values are conservative, as the suction flow rates are $10 \%$ higher than for minimum drag; added to this, they have been obtained by doubling the upper surface values (as no data for the lower surface was available). The profile drag for the fully laminar flow is approximately a fiftieth of the turbulent case, whilst the pump drag for the fully laminar aerofoil is more than $80 \%$ of the total drag. The total drag reduction is $89 \%$ : confirming the potential of laminarised surfaces. Green (2006) comments 


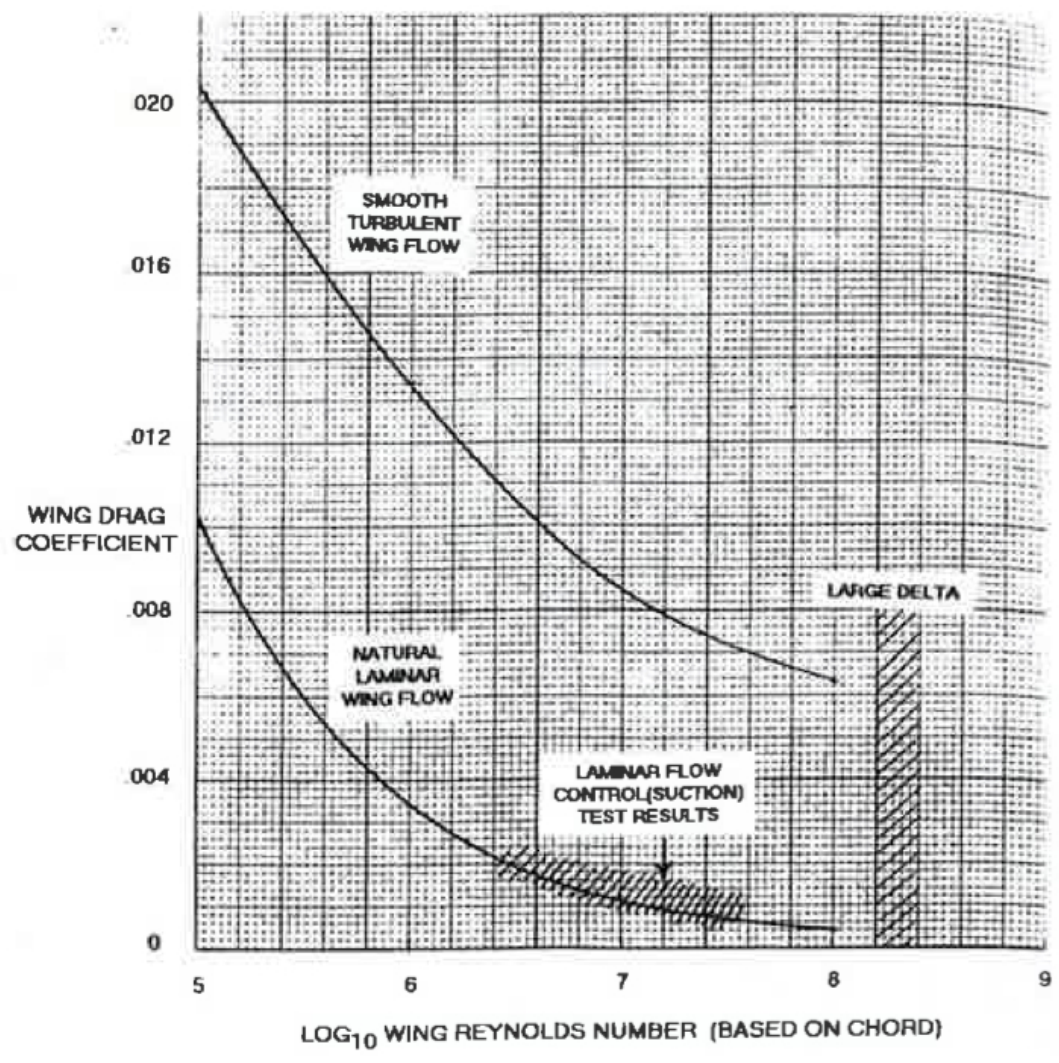

Figure 2.4: Variation in wing drag coefficient with chord Reynolds number for laminar boundary layers (from Denning et al. (1997)).

that the same result is likely to apply for a fully laminar aircraft. Lachmann (1955) even goes to the extent of suggesting that, to a first approximation, the profile drag of laminarised surfaces may be neglected altogether.

Table 2.1: Aerofoil drag comparisons on the F-94A upper surface, all for $R e_{c} \approx 30 \times 10^{6}$ (from Green (2006)).

\begin{tabular}{lcc}
\hline \hline & Fully turbulent & Fully laminar \\
\hline Profile drag & 0.00912 & 0.00018 \\
Pump drag & 0 & 0.00078 \\
\hline Total drag & 0.00912 & 0.00096 \\
\hline \hline
\end{tabular}




\subsubsection{Flow Control Design Methodology}

The basic approach to the design of LFC wings is illustrated in Fig. 2.5. It is first necessary to design the basic aerofoil section to meet any aircraft stability requirements and geometric constraints. With the baseline wing design, the wing pressures and velocities, calculated using transonic wing theory or computational fluid dynamics, can then be used in a boundarylayer stability calculation to determine the minimum suction requirements for laminar flow maintenance to the desired transition location. If suction requirements are unacceptably high then, depending on the most dominant instability mode and its region, the aerofoil wing section is adjusted accordingly, whilst still being capable of meeting other design constraints. The process is iterative and ultimately there will be a trade-off between aerofoil section characteristics and required suction flow rates.

With the desired suction flow rate determined, it is possible to proceed with the LFC system design. Joslin (1998a) outlines that the pressure drop through the skin must be set to a obtain a reasonable subsurface compartmentation and perforation spacing distribution for the desired suction distribution; furthermore, the ducts must be arranged and sized to minimise pressure losses. Finally, the compressor requirements can be used to estimate the demanded suction power.

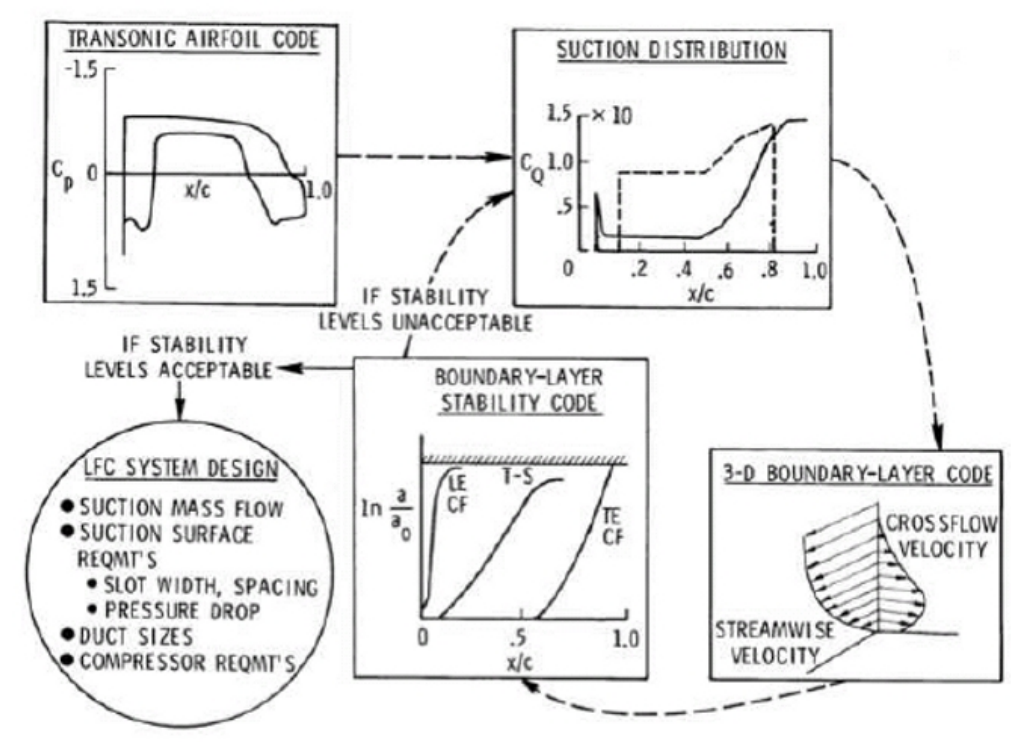

Figure 2.5: Block diagram of LFC design cycle (from Harris et al. (1988)). 


\subsubsection{Boundary Layer Stability}

\subsubsection{Tollmien-Schlichting Waves}

In two-dimensional boundary layers, Tollmien-Schlichting (T-S) waves are the primary instability mechanism. Saric and Reed (2006) describe these as essentially two-dimensional convective travelling wave disturbances generating streamwise instabilities that are slow growing. This instability is influenced by the shape of the non-dimensional boundary-layer velocity profile, which Hall et al. (1984) explain is due to the associated effects on the distribution of vorticity within the layer and viscous effects due to the change in Reynolds number. Green (2006) explains that, for high Reynolds number flows, these disturbances can be amplified even for profiles which do not have an inflection point; whilst for low Reynolds number flows small disturbances can be damped out.

\subsubsection{Taylor-Görtler Vortices}

Over the concave section of the lower surface of a cambered aerofoil, Taylor-Görtler vortexinduced instabilities dominate. These are stationary, streamwise oriented, counter-rotating vortices, as shown schematically in Fig. 2.6. Saric and Reed (2006) comment that this mechanism can easily be controlled through the use of convex curvature, whereby vortices of the opposite sign are generated, and are more stabilising than those produced by the concave curvature are destabilising; furthermore, they become less significant the larger the sweep angle is.

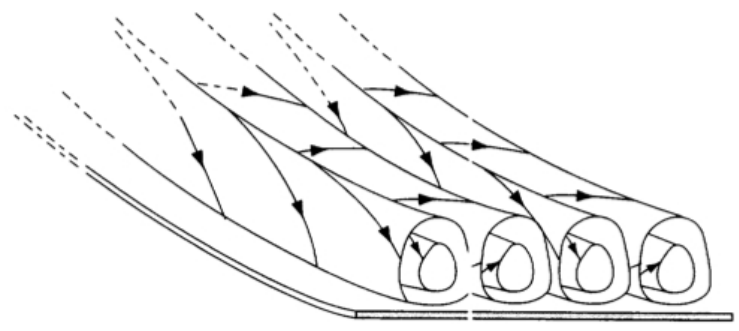

Figure 2.6: Schematic diagram of Taylor-Görtler vortices (from Joslin (1998b)).

\subsubsection{Crossflow Instabilities}

The crossflow $(\mathrm{CF})$ instability appears as co-rotating vortices, which all rotate in the same direction and whose axes are aligned to within a few degrees of the inviscid streamlines, as shown in Fig. 2.7(a). They dominate in regions where the flow is either accelerating or 
decelerating, as both the surface and flow streamlines are highly curved. The combination of wing sweep and pressure gradient deflect the inviscid-streamlines inboard (in a manner shown in Fig. 2.7(a)), which, when combined with the low momentum fluid in the boundary layer, makes the deflection even larger (Reed and Saric (1989)).

Figure 2.7(b) shows that there is an inflection point in the crossflow velocity profile: at the wall the no-slip condition is satisfied, whilst the crossflow velocity component in the free stream is zero. This causes a strong primary instability which depends on the crossflow Reynolds number, defined as $R e_{C F}=W_{\max } \delta_{10} / \nu$ - the boundary layer thickness $\delta_{10}$ corresponds to the point where the the crossflow velocity is at $10 \%$ of its maximum value (Reed and Saric (1989)).

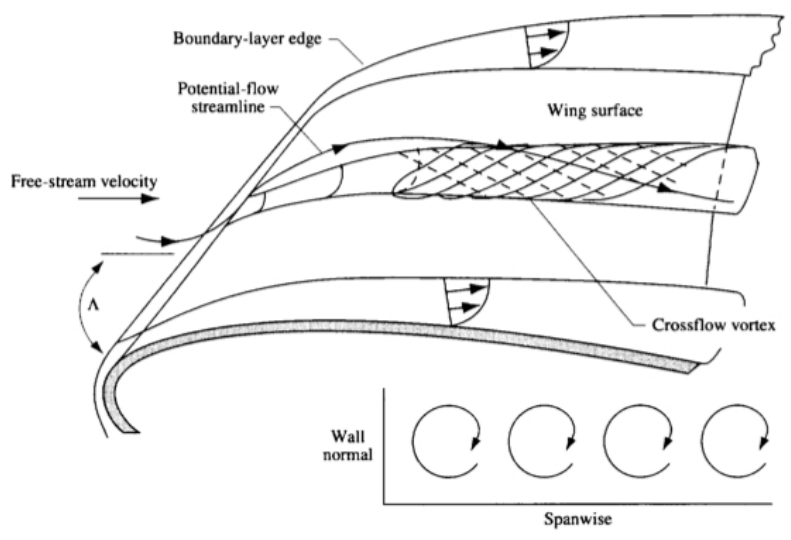

(a) Crossflow instabilities (from Joslin (1998a))

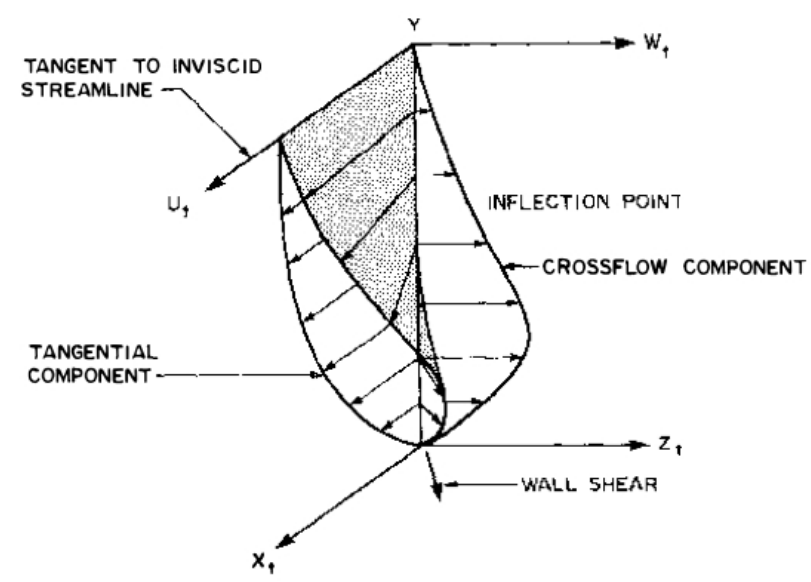

(b) Velocity components within a swept boundary layer (from Reed and Saric (1989)).

Figure 2.7: Schematic diagram of the crossflow instability and velocity profile. 


\subsubsection{Attachment-Line Instabilities}

A further issue in swept-wing transition is the stability of the 'attachment-line' boundary layer. This instability develops along the 'stagnation line' and causes transition in the leading edge region. It is shown schematically in Fig. 2.8.

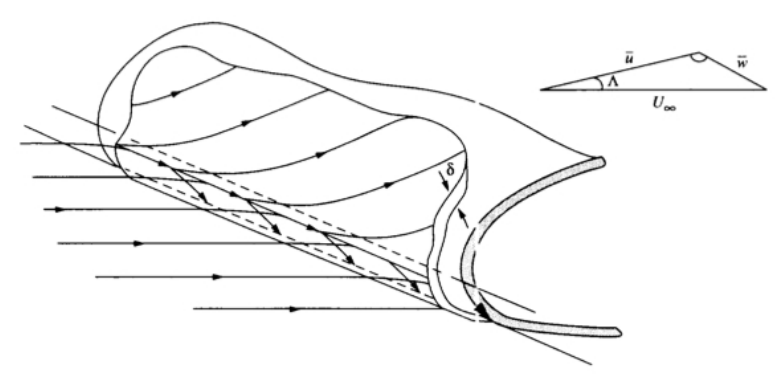

Figure 2.8: Schematic diagram of attachment-line flow (from Joslin (1998b)).

By considering an infinite swept-wing attachment-line boundary layer in a low turbulence free stream, Poll (1979) derived a governing Reynolds number dependent upon edge conditions and a characteristic length (which in turn depends on the chordwise velocity gradient), given by

$$
R e_{a . l}=\left(\frac{V_{e}^{2} c}{\nu_{e} U_{\infty} U_{\perp}}\right)^{\frac{1}{2}}
$$

where $U_{\perp}=\frac{c}{U_{\infty}}\left(\frac{d u_{e}}{d s}\right)_{s=0}$. The critical value of this attachment-line Reynolds number beyond which transition occurs was experimentally determined by Poll (1979) to be in the range 600 -700 .

Taking Eq. 2.7, and considering the potential-flow solution for a cylinder, Poll (1979) showed that, for this case,

$$
R e_{a . l}=\left(\frac{U_{\infty} r}{\nu} \times \frac{\sin \Lambda \tan \Lambda}{2}\right)^{\frac{1}{2}}
$$

In Eq. 2.8 we see that there is an explicit dependence on unit Reynolds number, leading-edge radius and wing sweep. Saric and Reed (2006) add that, for most applications, reduction of the nose radius for a specific sweep angle is a practical form of control. (This also has the compounded benefit of decreasing the chordwise extent of the crossflow region and providing a more rapid acceleration of the flow over the wing.) 


\subsection{LAMINAR FLOW CONTROL}

\subsubsection{Shock Wave / Laminar Boundary Layer Interactions}

During flight testing of the F-94A a new potential problem appeared. As the aircraft speed was increased to the point where the local Mach number on the wing surface exceeded about 1.10, full-chord laminar flow was lost with the slot configuration tested (Braslow (1999)). The problem was attributed to the steep pressure rise through the shock waves that formed. In more recent experiments performed by Harris et al. (1988), much effort was put into obtaining a shock-free supercritical aerofoil with a large supersonic zone of over $80 \%$ of the upper surface. The maximum local Mach number was recorded to have reached about 1.11 - greater in both extent and magnitude than those measured on the F-94A. A problem with the aerofoil, however, was that it had a very narrow off-design operating range.

Harris et al. (1988) reviewed some of the earlier work on this and found that suction laminarisation only appears practicable in regions of weak shocks on transonic aerofoils; moreover, the pressure rise that a laminar boundary layer can negotiate in the region of an incident shock-wave decreases with increasing Reynolds number, unless the thickness of the upstream boundary layer can be reduced in some manner, for example, by suction. However, Harris et al. (1988) conclude that quantification for boundary layer control in such shockinteraction regions still requires experimentation.

\subsubsection{Example Suction Distributions}

As we have seen, the presence of T-S and CF disturbances in the boundary layer are primarily dependent on the pressure gradient and wing sweep angle. However, Joslin (1998b) comments that for wings with sweeps between $0^{\circ}$ and $25^{\circ}$, T-S wave instabilities dominate; whilst for wing sweeps greater than $25^{\circ}$ both T-S and CF disturbances are present; and for even greater wing sweeps of above $30^{\circ}$ to $35^{\circ}$, CF disturbances alone dominate near the wing leading edge.

An example chordwise suction distribution necessary for laminarisation of the $30^{\circ}$ swept $\mathrm{X}-21 \mathrm{~A}$ aircraft is shown in Fig. 2.9. (The stability calculations were performed assuming incompressible laminar boundary layers for flow over an infinitely swept wing. The calculated suction distribution is compared against that measured during flight tests in the figure, and agrees reasonably well.) The pronounced suction over the leading edge is due to a combination of strong crossflow instabilities in the favourable pressure gradient region and attachment-line instabilities. (In order to prevent attachment-line contamination from the turbulent fuselage, a number of control techniques were employed: a fence, vertical slots, and a gutter.) Over the mid-chord, where there is a mild adverse pressure gradient, T-S waves dominate and these require a lower degree of surface suction for stabilisation. Toward the trailing edge, the increased adverse pressure gradient results in a higher requirement, due to a combination of 
T-S and CF instabilities.

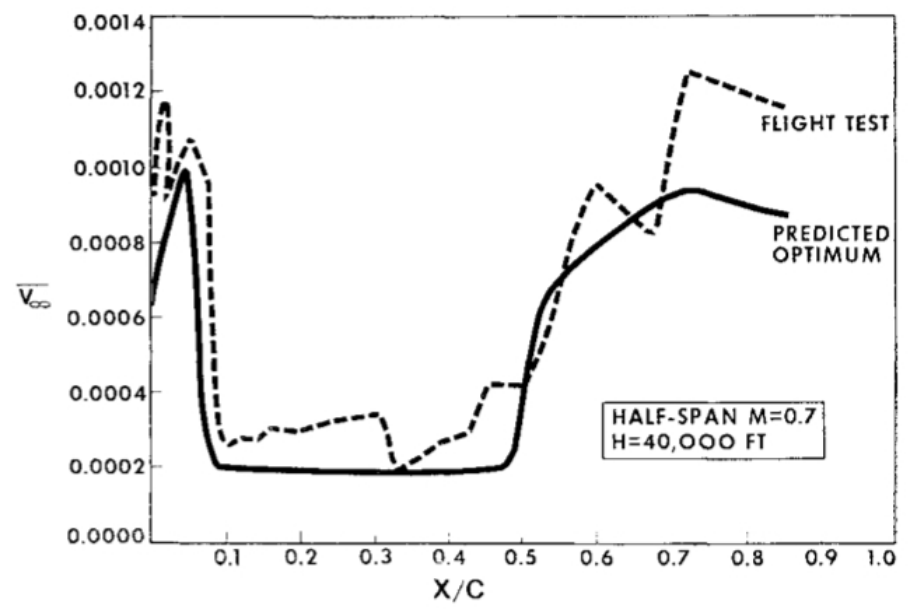

Figure 2.9: Example suction flow velocity distribution for the $30^{\circ}$ (quarter-chord) sweep X-21A aircraft (Joslin (1998a)).

In contrast, we can see in Fig. 2.10 that the shape of the suction distribution calculated for the Vampire, with a quater-chord sweep of $10^{\circ}$, differs considerably. Suction stabilisation against crossflow instabilities over the front of the aerofoil is absent given the reduced wing sweep, whilst the requirement increase over the rear of the aerofoil, due to the adverse pressure gradient, is much further downstream.

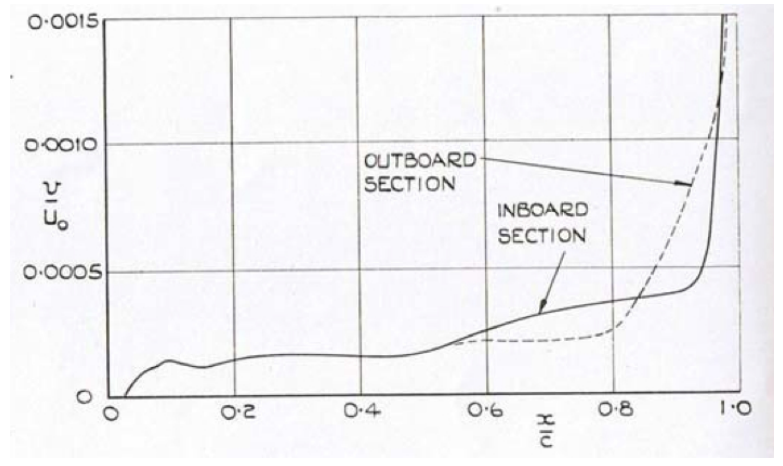

Figure 2.10: Example suction flow velocity distribution for the $10^{\circ}$ (quarter-chord) sweep Vampire aircraft (from Head (1955)).

In a review of the historical aspects of LFC, Denning et al. (1997) quote Lachmann, who comments that only $5-10 \%$ extra suction flow is required on a swept planform relative to an unswept one. Quantification for a specific case confirming this does not seem to be available in the open literature. 


\subsection{LAMINAR FLOW CONTROL}

\subsubsection{LFC System Design Considerations}

Figure 2.11 shows, schematically, the key elements of a LFC suction system. The boundary layer flow, with a total pressure equal to the local static pressure, is sucked across a porous skin into a set of corrugations and transported along a set of ducts, through which additional losses are incurred; it is then fed into a set of suction pumps, which are required to increase the total pressure of the flow and discharge it rearwards.

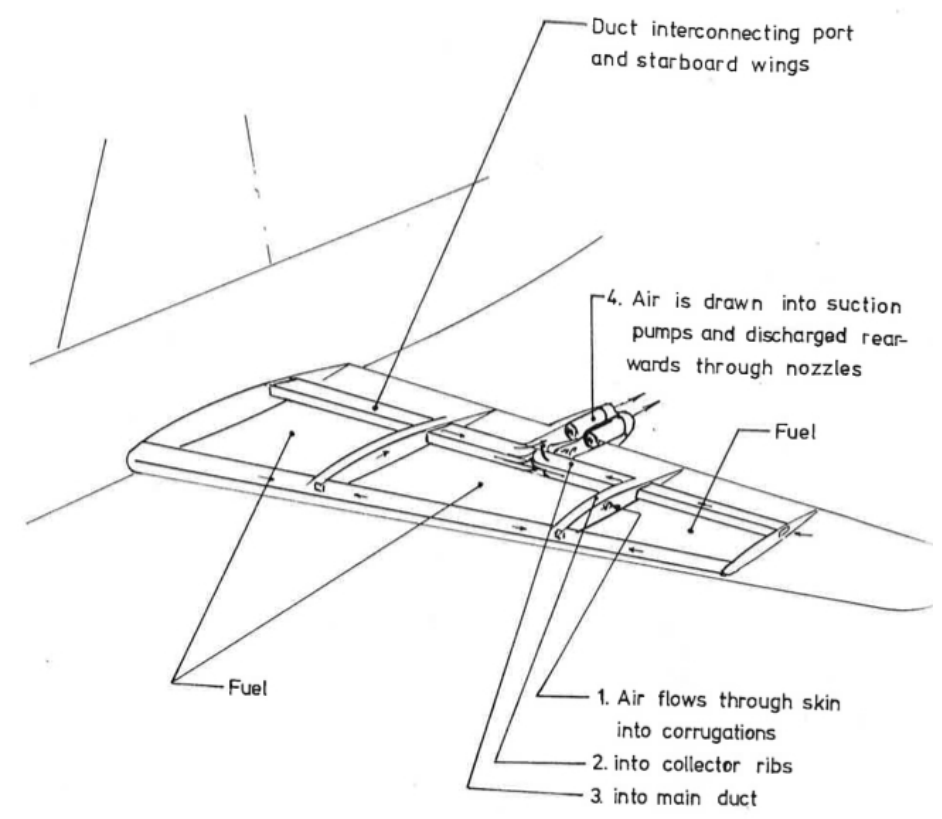

Figure 2.11: Outline for a laminar flow control system (from Edwards (1977)).

Pearce (1983) comments that a practical suction system implementation must be light weight and should minimise the fuel consumed by the LFC system; highlighting the pressure loss in the system as the critical parameter. For this reason, previous studies of the required power have mainly focussed on trying to minimise the suction-system losses. In summarising research conducted at Northrop, Pfenninger (1977) considers throttling and mixing losses, flow separation, duct-wall friction and secondary-flow losses, and proposes a number of novel solutions to minimise them. However, compared to the losses associated with the deceleration of the flow in the boundary layer, the additional contributions through the suction system seem fairly small. Lachmann (1955) and Gregory (1961) both state that the main effort should be in overcoming boundary-layer losses. Gregory (1961) also notes that low additional suction power demands a porous surface whose resistance to flow varies over the chord, in order to minimise unwanted variations; Head (1955) and Lachmann (1955) incorporated 


\section{CHAPTER 2. LITERATURE REVIEW}

such a resistance into throttle holes situated in a cellular subsurface structure, as shown in Fig. 2.12. A final strategy for minimising the additional power is suggested by Pearce (1983): in regions where the suction requirements are high, triangular chambers of full aerofoil section depth may be implemented to minimise losses in transporting the flow along the wing to the suction pumps. He also adds that higher losses can be tolerated over the rear of the aerofoil as the surface pressure is higher.

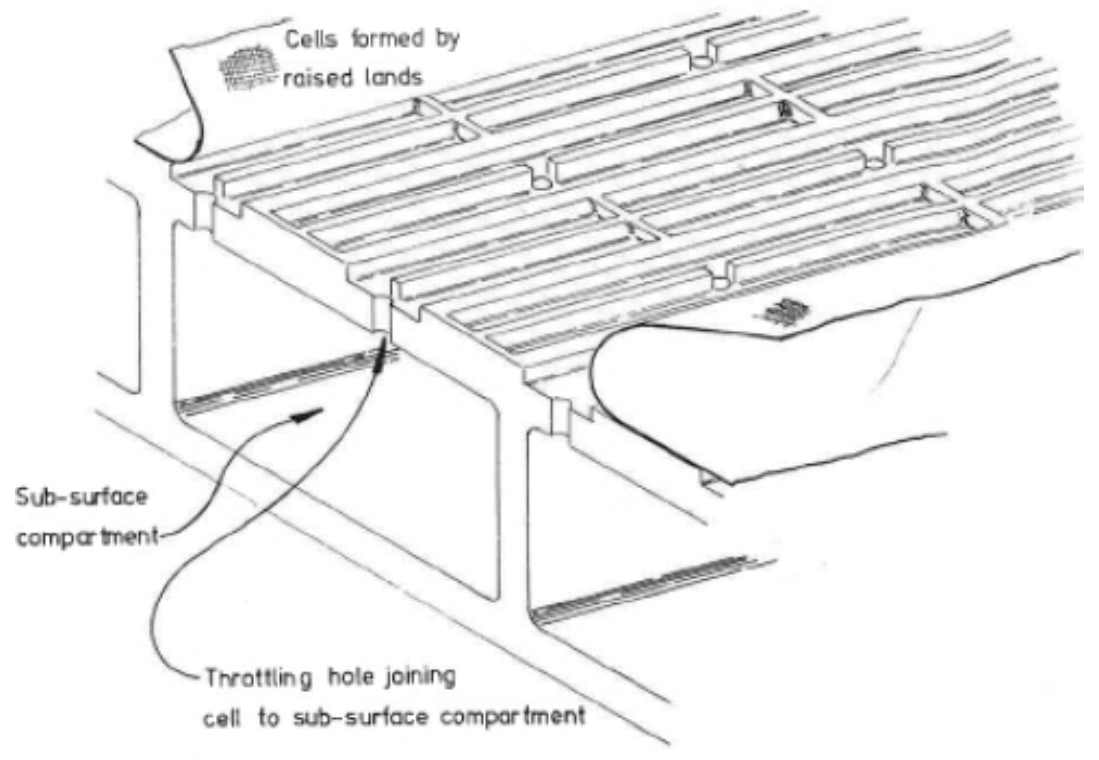

Figure 2.12: Typical suction surface (from Edwards (1977)).

Green (2006) explains that the pump power required to energise the suction system cannot be expressed as a simple function of the total suction quantity (as it can for inviscid flow, whereby the sucked flow is treated as sink drag), but has to be estimated carefully for each individual case. The pressure losses incurred through a typical suction system are detailed in Fig. 2.13. The loss in free-stream dynamic pressure and external losses due to the surface supervelocity distribution dominate the losses through the surface and ducting. The pump inlet total pressure is set at the lowest pressure in the system; however this leads to unneccessary additional losses for the flow over the rear of the aerofoil. Instead, Lachmann (1955) proposes that a multi-stage pump be utilised in order to grade the suction pressure.

Edwards (1961) proposes three schemes through which power may be delivered to the suction system. The first consists of a primary propulsion system delivering the power via a drive shaft. The second is an auxiliary engine, separate from the propulsion system, used to drive the suction pump via a drive shaft. The third is direct boundary layer ingestion by the 

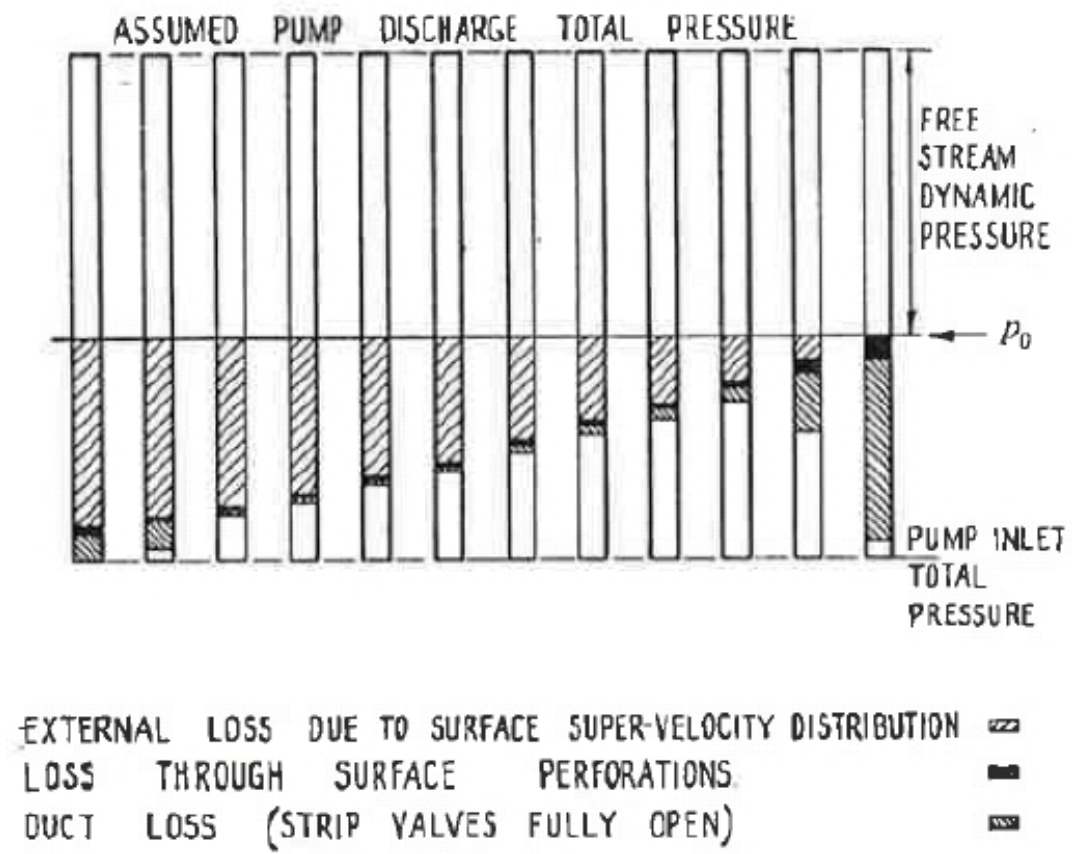

Figure 2.13: Various pressure loss components making up pump power (from Lachmann (1955)).

main propulsive units. Edwards (1961) comments that the second scheme should be applied to research aircraft, as it is only slightly less efficient than the first, and would not require the same extensive modifications to the primary propulsion system, whereas the third scheme simply has lower thermal and overall efficiencies.

Kosin (1965) provides some information with regard to the suction/propulsion system integration for the $\mathrm{X}-21 \mathrm{~A}$ flight test programme. The main turbojet propulsion engines were suspended on pylons at the rear of the fuselage to reduce noise and the ensuing turbulence level on the wing. The suction pumps for the LFC experiments were located in nacelles underneath the wing, and installed at about one-third half-span to keep the collector ducts short and duct velocities low. The suction pumps were driven by compressor bleed from the main propulsion engines - this combination effectively converts the turbojet into a turbofan engine, whereby the pumps act as fans increasing the bypass ratio. A schematic diagram of the system is provided in Fig. 2.14. In agreement with Edwards (1961), Kosin (1965) comments that if the pumping compressor were driven by its own independent powerplant a lower total efficiency would result.

Two suction pumps were utilised in the X-21A study: a small pump with a pressure ratio of 1.2 , and a large pump with a pressure ratio of 1.65 . The smaller pump sucks boundary 


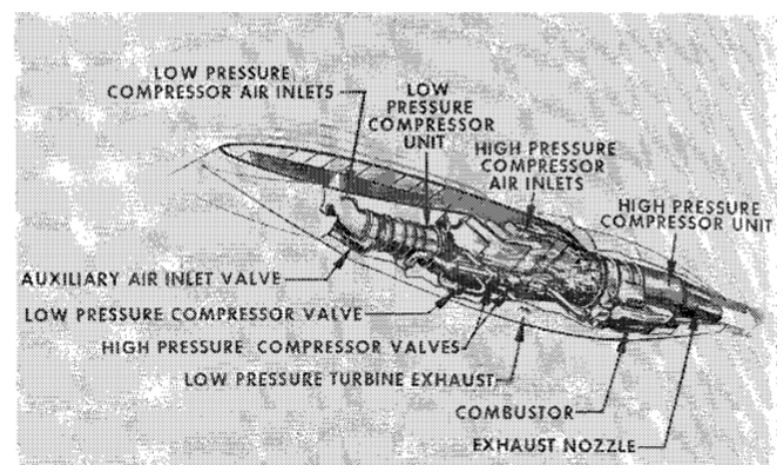

Figure 2.14: X-21A laminar flow pumping system (from Kosin (1965)).

layer fluid from the low-pressure region of the wing, and the flow is then discharged together with the remaining boundary layer fluid into a mixing chamber in front of the large pump. The total sucked airflow is then ejected through a nozzle at the end of the suction nacelle. (Lachmann (1955) comments that for most suction applications, the pumps will need to produce a pressure ratio no more than the order of $2.5-3.0$.)

Pfenninger (1987) explains that background disturbances coming from the engine can have a great affect on the stability of a laminar boundary layer. However, in a more recent technology assessment, Wilson (1997) concludes that the influence of wing mounted engines is minor, and is only an issue for the maintenance of laminar flow over the nacelles themselves, given the proximity of the noise source.

On the integration of the propulsion system with a suction-laminarised aircraft, Lachmann (1955) says: "The more cruising drag and thrust are reduced by progressive laminarisation, the more the ratio take-off to cruising thrust reaches uncomfortably large values, and the endeavour to substitute existing power plants by boundary layer propulsion must be tempered by take-off and climb considerations". He then goes on: "It is obviously uneconomical to carry an over-sized power plant over the whole range to the detriment of payload, the function of part of this power plant being restricted to relatively short duration". Consequently, Lachmann (1955) proposes that the engines be tailored to meet cruise conditions, but supported by another engine specifically designed to bolster the thrust at takeoff, in an effort to minimise the overall propulsion system weight.

\subsection{The Flying Wing Configuration}

The flying-wing configuration is an attractive concept in terms of its aerodynamics and structural weight, which arise due to a lower wetted area and span loading effects, respectively. 


\subsection{THE FLYING WING CONFIGURATION}

Furthermore, Martinez-Val (2007) explains that there is the potential for a quieter aeroplane, as engines may be located above the wing, whilst high-lift devices in the low-speed configuration are not required. It is mainly for these reasons that there has been a great deal of activity, at a conceptual level, in recent years.

However, research into the flying wing concept began over 60 years ago with the Horten brothers' sailplanes in Germany during the 1930's and 1940's. The Hortens were followed by John Northrop with the YRB-49 in the USA post-world war two. However, we are yet to see such an aircraft in service in the commercial aviation market. Weishaar and Ashley (1973) highlight two of the most prominent reasons for the failure of the early flying wings to evolve as operational aircraft:

1) a difficulty to achieve satisfactory unaugmented handling qualities, particularly in the lateral-directional modes;

2) and that the early FW's tested were simply not big enough.

This section begins with an overview of the structural architecture of the flying wing and various operational design considerations. Then the influence of key configuration parameters on the aircraft's stability and control characteristics are discussed. Finally, the flying wing's handling qualities and aeroelastic behaviour are reviewed.

\subsubsection{Structural Architecture}

The structural architecture of the FW is two-fold: the centrebody must accommodate passengers, whilst the outer wing has a more conventional structure providing volume for fuel tanks and cargo holds (Martinez-Val (2007)). In order to enable efficient use of the inner space for application to civil transport, aerofoils with large thickness-to-chord ratios are necessary as passengers must be capable of standing upright; otherwise, very long chord lengths are necessary; for example, Liebeck (2004) and Martinez-Val (2007) consider 17 percent thick aerofoils. Although aerodynamically challenging, Bolsunovsky et al. (2001) comment that the increased structural depth makes it possible to increase the wing span with less weight penalty compared to conventional aircraft.

With the payload located inside the wing, the centrebody structure must resist pressurisation in addition to ordinary bending and torque moments induced by external aerodynamic and inertial loads in its function as a wing. Martinez-Val (2007) comments that the loads combine in a nonlinear manner, which under extreme maneouvres or gusts can produce significant stresses and deformations.

Liebeck (2004) considers two structural concepts for the centrebody pressure vessel in which the skin would be capable of resisting all prescribed loads: 1) a separate pressure 


\section{CHAPTER 2. LITERATURE REVIEW}

shell; and 2), an integrated skin and shell structure. These are illustrated schematically in Fig. 2.15.
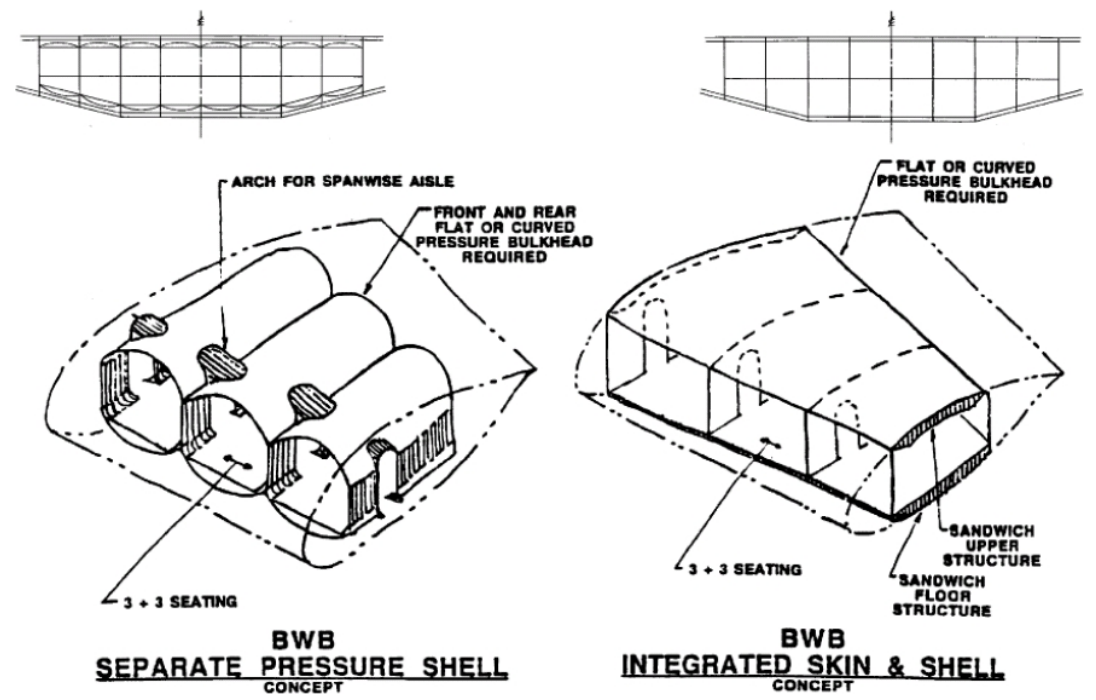

Figure 2.15: Structural concepts for the centrebody pressure vessel (from Liebeck (2004)).

Having a separate pressure shell divides the load carrying responsibility of the two major structures. The arched pressure vessels are used to react the pressurisation loads in tension, and the conventional outer skin bears the additional loads. Liebeck (2004) comments that a potential safety issue with this concept is that if a rupture were to occur in the thin arched skin, the cabin pressure would have to be borne by the wing skin, and therefore must be sized accordingly with a corresponding increase in structural weight. However, as Martinez-Val (2007) comments, the inner vaulted skin effectively takes the equivalent responsibility as the fuselage structure in conventional aeroplanes, which are sized to resist quite large ruptures. A problem with the second configuration is that the outer skins are made up of quasi-planar panels, which are very inefficient in resisting internal pressure loads. With both concepts, ribs are necessary to carry concentrated shear forces, contribute to the torsional stiffness of the structure and maintain the external aerodynamic shape (Martinez-Val (2007)). As far as the passengers are concerned, i.e. headroom and passages, there are no major differences. Bolsunovsky et al. (2001) comment that a separate pressure shell would allow the placement of various items, such as the LFC hardware, in between the inner and outer skins. Other concept selection criteria may include direct operating costs, which are mainly influenced by manufacturing and maintenance issues. However, according to Martinez-Val (2007), no studies have been carried out on the influence of such considerations.

For conceptual and preliminary design studies, initial weight estimates are commonly 
attained using correlations based on historical data of existing aircraft. The applicability of such approaches to aircraft of an unconventional configuration is somewhat suspect. To take full account of aircraft geometry and attain greater accuracy (at the cost of complexity), finite-element methods are available, but are usually reserved for the detailed design phase. A third, intermediate, option is to size primary structural elements by treating them as simple geometric shapes subject to the necessary loading conditions, using standard analytical methods. The TASOPT and PDCYL aircraft structural design modules discussed by Greitzer et al. (2010) and Ardema et al. (1996), respectively, employ the latter technique; both modules have been developed for the analysis of new aircraft configurations that assume non-conventional materials, but still retain the 'tube and wing' layout.

\subsubsection{Cabin Arrangement and Operational aspects}

Figure 2.16 shows a cabin arrangement that takes the form of a set of parallel bays. MartinezVal (2007) argues that this arrangement allows seats to be classed according to comfort levels in rough flights, because those seats further outboard are more likely to experience ride comfort troubles with regard to lateral and vertical g-loads about the roll axis. (However, he comments that unpleasant rolling accelerations could be balanced by smooth manoeuvres and multimedia equipment.)

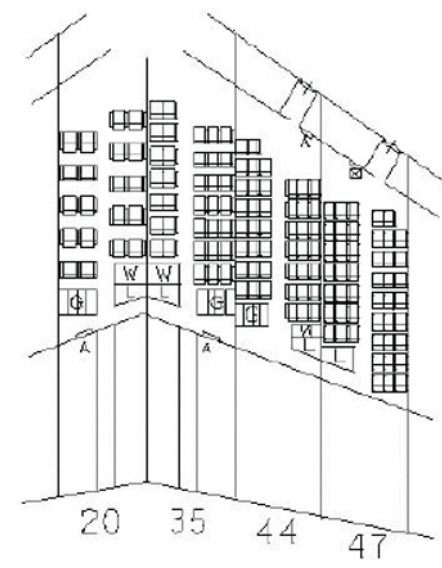

Figure 2.16: Cabin seating arrangement (from Martinez-Val (2007)).

Aeroplane operation concerns on-ground manoeuvres and servicing, and emergency evacuation. In the FW design study of Bolsunovsky et al. (2001), great attention is paid to the following operational aspects:

- structure and logistics of emergency exits; 


\section{CHAPTER 2. LITERATURE REVIEW}

- service compartment arrangement e.g. galleys etc.;

- cabin floor inclination at cruise;

- waterline ditching and provision of ramp slides;

- landing gear and wheel base track;

- tip-over on taxiing;

- centre-of-gravity build-up - loading sequence, positioning, fuel tank capacity and usage sequence.

In respect of runway/taxiway movements or airport terminal operations, Martinez-Val (2007) argues that there are no significant differences between FW and conventional aircraft.

The locations of doors/emergency exits depend on a number of factors, and are discussed by Martinez-Val (2007). For example, placement along the leading edge demands additional structural reinforcement to ensure integrity during a crash or object strike, whilst also requiring provisions to avoid cabin flooding in the event of a ditch. In contrast, the placement of exits through the rear poses fewer problems. Alternatively, Lee (1961) proposes passenger entry to the cabin via the landing gear hatches on the underside of the aircraft, with passenger escape hatches located on the top surface. Another consideration is that the doors must also be arranged to allow access to the aircraft independently for galley servicing and cleaning.

Emergency evacuation is another important consideration. However, Martinez-Val (2007) concludes that, although the flying wing does suffer from having an extra wide body, it does not pose any serious problems in respect of passenger flow rate and total evacuation time.

Maximising on ground servicing access is important as it enables a shorter turn-around time, which, for current aircraft, is typically around 45 minutes (Martinez-Val (2007)). With galleys often located at the rear for aesthetic and evacuation purposes, the front doors may be used for cabin cleaning and servicing access. Martinez-Val (2007) adds that in this situation passenger services, cargo/baggage handling, and aeroplane servicing can be done simultaneously with an overlap of activities. Another consideration is the loading and unloading of passengers in airport piers, which requires the floor to be positioned a certain height above ground level - for current aircraft this is around $5 \mathrm{~m}$.

Martinez-Val (2007) comments that with regard to airport infrastructure, there is no real justification for having an aircraft of span greater than $80 \mathrm{~m}$ even for entry in the mid-term future, as all major airports have recently made major modifications to aircraft boxes to 


\subsection{THE FLYING WING CONFIGURATION}

accommodate the A380 and other potential ultra high capacity aeroplanes. Furthermore, runway widths are currently at $14 \mathrm{~m}$.

The provision of windows is restricted to the leading edge, and this has been highlighted as a potential concern by aircraft manufacturers. With LFW aircraft, the positioning of windows is even more restrictive as they cannot be laminarised. On this point, Lee (1961) proposes the use of multi-media equipment to reduce feelings of claustrophobia.

\subsubsection{Stability and Control Considerations}

In steady flight, an aircraft is said to be in a trimmed state if there are no resulting turning moments about any of the aircraft's axes. An aircraft has six degrees of freedom that lead to the following three stability criteria about each of the three axes:

- Longitudinal stability: $C_{m_{\alpha}}<0$ - a form of pitching stability about the lateral axis whereby the aircraft tends to pitch down after an increase in $\alpha$.

- Directional stability: $C_{n_{\beta}}>0$ - also known as 'weathercock stability', refers to the yawing stability about the vertical axis.

- Lateral stability: $C_{l_{\beta}}<0$ - refers to the rolling stability about the longitudinal axis.

Lateral and directional stability are closely interconnected, with the generation of an adverse yaw during a roll manoeuvre. Therefore, these two areas will be discussed simultaneously.

A measure of longitudinal static stability is given by the static margin $\sigma_{L}$, defined as:

$$
\sigma_{L}=\frac{x_{N P}-x_{C G}}{c}
$$

where $x_{N P}$ is the neutral point (NP). The NP is the chordwise location at which the aerofoil section only sees a change in lift coefficient with angle of attack whilst the pitching moment coefficient remains constant, and equal to the zero-lift pitching moment coefficient $C_{M 0}$. If the NP lies aft of the C.G., $\sigma_{L}>0$ and the aircraft exhibits longitudinal static stability.

In addition to static stability, inertial and time-dependent effects govern an aircraft's dynamic stability. This characterises any unsteady motions whereby after a disturbance, the aircraft may first return back to its original state and is therefore statically stable, but then progressively larger oscillations may build up around that state of equilibrium.

\subsubsection{Longitudinal Stability}

As for conventional aircraft, Donlan (1944) concludes that the longitudinal static stability of a FW is assured if the C.G. lies ahead of the NP. However, once the C.G. is allowed to shift 
to a position behind the NP, Jones (1941) highlights that the rate of divergence of a tailless aeroplane is more rapid, and may become uncontrollable even at small distances.

Although a positive static stability margin is desired, Donlan (1944) explains that it should not be too great as excessive elevator control power might be required during take-off, whilst the resulting large deflection of the elevator would impair the aerodynamic performance of the wing. Bolsunovsky et al. (2001) investigated the limits of fly-by-wire control systems technology and found that during the landing and takeoff regimes a static margin close to zero was possible, whilst in cruise flight $d C_{m} / d C_{L} \leq 0.03$ should not be exceeded.

An important stability characteristic of tailless aircraft relative to conventional aircraft arises due to a reduced kinematic damping of the pitching motion, $C_{m_{q}}$. Jones (1941) explains that this phenomenon is mainly because of the absence of the tail surface, with the effect of the fuselage shown to be unimportant. Jones (1941) comments that if the FW also has a short static margin, it is likely to be more sensitive to pitch control giving a much increased manoeuvrability.

Nevertheless, for a statically stable tailless aircraft, free rotations in pitch are coupled with motions normal to the chord. Consequently, additional damping is obtained through this coupling, as the vertical damping parameter $C_{Z_{w}}$ absorbs most of the energy of the oscillation. The lack of direct damping appears to alter the sequence of the motions in such a way as to make this coupling more effective in the case of a tailless aeroplane. Therefore, in spite of the low direct pitch damping, Donlan (1944) and Northrop (1957) found the short period oscillation (SPO) instability to be highly damped for tailless configurations and as great as that for conventional aircraft. Finally, Northrop (1957) comments that any differences in the phugoid damping with respect to conventional aircraft are insignificant as it is a slow motion that can easily be controlled.

Both Nickel and Wohlfart (1994) and Donlan (1944) describe a form of dynamic instability known as tumbling, which is observed with tailless aircraft but is not a particular issue for conventional aircraft. Tumbling manifests itself as a continuous automatic rotation about the pitch axis. The only occurrence of tumbling as noted by Donlan (1944) was that simulated in a free-spinning tunnel. A large static margin helps prevent it arising. If the static margin does become negative, then there is a danger of either encountering a divergence in pitch attitude from straight flight or tumbling. However, Donlan (1944) contends that tumbling would never arise during a normal flight condition, whether it be as a result of a stall or a spin. 


\subsection{THE FLYING WING CONFIGURATION}

\subsubsection{Lateral-Directional Stability}

The provision of sufficient weathercock stability and damping in yaw proves to be the main difficulty, due to the short available moment arm for control. Northrop (1957) comments that the swept-back wing has inherent directional stability, which increases with $C_{L}$, but is alone not sufficient for satisfactory flight characteristics under all circumstances, thus requires wingtip fins.

A consequence of the FW's inherent weak damping in yaw and corresponding poor directional stability is the increased resulting strength of the adverse yaw associated with a roll manoeuvre. If coupled with a positive skid-roll moment, Nickel and Wohlfart (1994) highlight that the effectiveness of the ailerons for lateral control may be destroyed. It is therefore important to eliminate this strong adverse yaw. Jones (1941) suggests that an autopilot, used to provide directional control, could be linked with the aileron control to minimise the effects of adverse aileron yaw; however, the problem may also be countered through geometric considerations.

Particularly at low speeds, high weight and high altitude, Dutch roll is comparatively bad for tailless aircraft because of the combination of a large effective dihedral, $C_{l_{\beta}}$, and low weathercock stability $C_{n_{\beta}}$ (Northrop (1957)). Furthermore, if the rudder is particularly weak, the time of response to rudder control may be of the same order as the period of the Dutch roll. This makes directional control especially difficult in landing conditions, where the roll controls are not usable for changing heading. Another factor adding to the weak damping of the Dutch roll is the low value of yaw damping $C_{n_{r}}$. However, as was the case for damping in pitch, both Jones (1941) and Donlan (1944) found that, in general, the damping of the lateral oscillations is greater than indicated from the damping due to yawing velocity alone. Thus the small value of $C_{n_{r}}$ is not detrimental to the flying qualities, provided that adequate directional stability is provided; furthermore, Dutch roll is of a rather long period, therefore it is typically not necessary to have a high rate of damping as control is usually easy.

Associated with the low values of $C_{n_{r}}$ is some concern over large angles of sideslip developing when the aircraft is subjected to an asymmetric loss in thrust. However, referring to wind-tunnel tests, Donlan (1944) comments that the effects of $C_{n_{r}}$ are "probably secondary to other parameters". Furthermore, Donlan (1944) explains that the maximum amplitude of the sideslip oscillation is markedly influenced by the rolling moment due to sideslip $C_{l_{\beta}}$ and the yawing moment due to sideslip $C_{n_{\beta}}$. On this point Donlan (1944) concludes that increasing either the directional stability or the dihedral reduces the magnitude of the sideslip generated by a yawing moment; however, observes that the former gives the greater reduction.

Donlan (1944) and Northrop (1957) both point out that the steady-spin characteristics 
are comparable to those of conventional aircraft. However, Nickel and Wohlfart (1994) add that, in fact, tailless aircraft are probably less spin prone because the primary forces which influence spinning are gyroscopic, therefore a distribution of masses lying far away from the $\mathrm{CG}$, as for flying wings, increases the rotational resistance of the aircraft.

On the spiral mode stability, Northrop (1957) comments that tailless aircraft have readily acceptable characteristics in this mode.

\subsubsection{Control}

The longitudinal and lateral controls are combined into elevons, which operate together for pitch control and differentially for roll control. To realise the advantages of a differential aileron system, a two control surface combination is required, as illustrated in Fig. 2.17. Nickel and Wohlfart (1994) explain that the outer surfaces work differentially as ailerons, whilst the inner surfaces not only support the aileron effect, but also take care of the balance of pitching moments about the lateral axis. For the example here, the inner surface on the left is more deflected than the outer surface on the right because it has to balance the pitching moments at a shorter lever arm due to the sweep back.

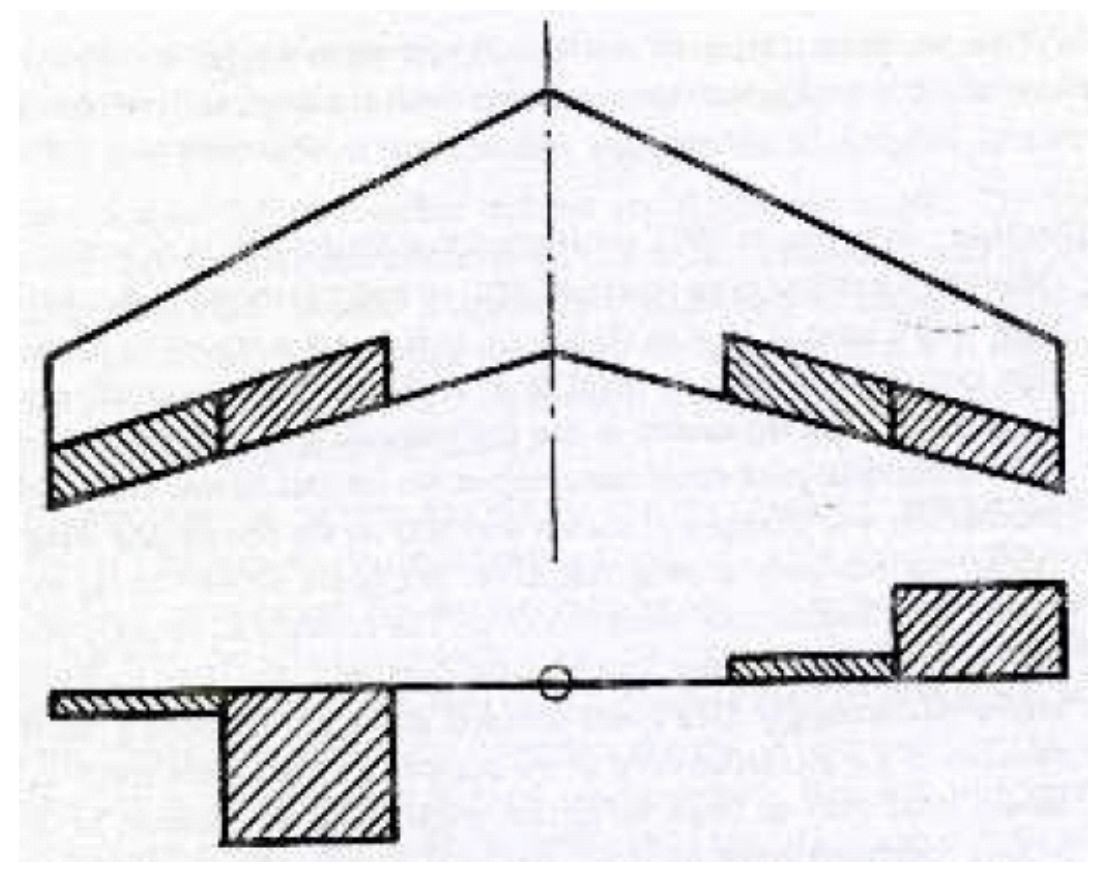

Figure 2.17: Differential elevon control for a pure aileron deflection to the right without any elevator effect (from Nickel and Wohlfart (1994)).

Trim control is achieved on tailless aircraft with the aid of elevator control surfaces located 


\subsection{THE FLYING WING CONFIGURATION}

at the trailing edge of the wing. To produce the same change in $C_{m}$, the elevator of a tailless aircraft must be deflected more than that of a conventional aircraft of the same static margin. With aircraft generally having an overall nose-down pitching moment, upward deflection of these surfaces generates down-lift over the trailing edge, increasing the profile moment of the wing section. This not only reduces the local section $C_{l}$ but also $C_{L, \max }$, worsening as the static margin increases (Nickel and Wohlfart (1994)). However, Northrop (1957) comments that the required elevator trim will be less for tailless aircraft with close to neutral static stability.

Northrop (1957) chose to eliminate vertical fin and rudder control surfaces, firstly because they violate the all-wing principle and add drag to the basic aerofoil; whilst secondly, for designs incorporating a moderate amount of sweepback, the moment arm of a conventional rudder about the CG is small and an excessively large rudder would result when aiming to achieve conventional yaw control moments. However, Nickel and Wohlfart (1994) comment that wingtip fins are not only useful for steering but also increase directional stability, whilst decreasing induced drag. Nevertheless, Northrop (1957) concentrated on trying to find a type of drag-producing device at the wing tips that would give adequate yawing forces, without affecting pitch or roll. The drag needed is not very large since it works over quite a long lever arm, about half-span.

A double-split flap at the wing tip was found to have the most satisfactory all-round characteristics, whilst allowing for the combination of a trim flap and rudder in the same portion of wing trailing edge; however, it does suffer from low effectiveness at low angles of rudder deflection, and in addition Nickel and Wohlfart (1994) comment that besides increased construction complexities, the departing vortices associated with double-split flaps often induce vibrations on the flaps. Nevertheless, the double-split flap was adopted by Northrop on the B-2 bomber, as illustrated in Fig. 2.18. ${ }^{1}$.

Spin recovery is generally accomplished by deflecting the ailerons such that a rolling moment is generated to counter the spin. However, Donlan (1944) explains that wingtip fins are also effective for spin recovery, especially if the rudder extends below the wing in order to overcome/reduce the blanketing effects of the wing. In contrast, drag rudders were not found to be very successful due to the varying nature of performance of those located in stalled and unstalled regions - in some cases they were even found to produce pro-spin rolling moments.

Rudder control is not only necessary to counter the effects of adverse yaw, but also to trim the aircraft directionally under asymmetric power conditions. Donlan (1944) proposes that, to reduce the rudder control requirements, the thrust lines should be located as close as

\footnotetext{
${ }^{1}$ http://upload.wikimedia.org/wikipedia/commons/thumb/b/be/USAF_B-2_Spirit.jpg/600px-USAF_B2_Spirit.jpg
} 


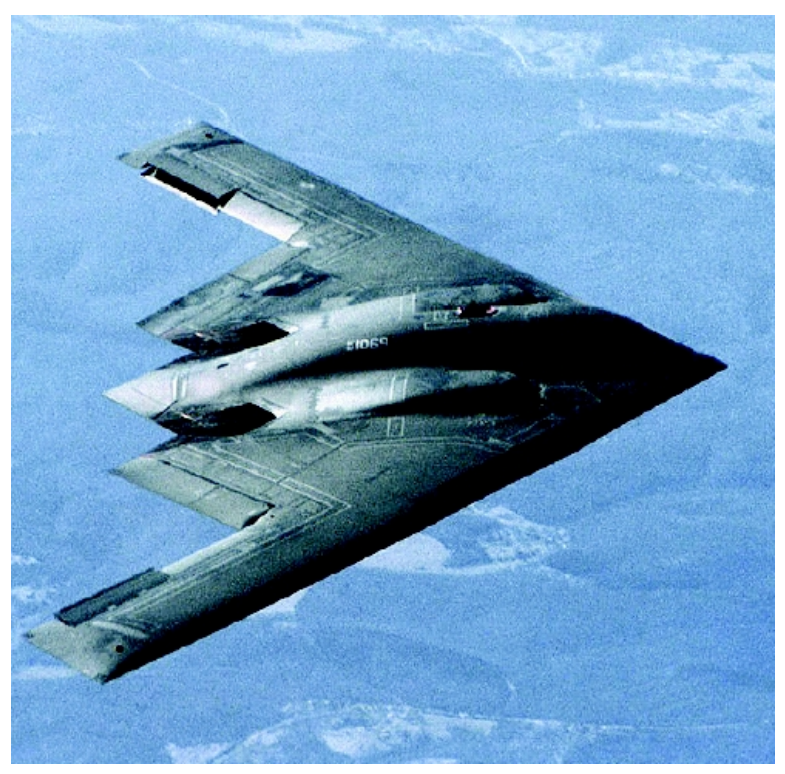

Figure 2.18: B-2 split flap rudders.

possible to the centreline and ailerons that create favourable yawing moments when deflected should be adopted. Bolsunovsky et al. (2001) also suggest the use of split flap elevons in addition to vertical rudder control surfaces in order to provide extra yaw control.

\subsubsection{Effects of Wing and Planform Geometry on Stability and Control}

\section{Sweep}

Figure 2.19 shows that sweeping the wing significantly changes the lift distribution. A 'lift valley' results toward the centreline, whilst a 'lift mountain' develops toward the wing tips, becoming more pronounced as the angle of sweepback increases. (Sweeping the wings forward has the opposite effect; however, this is not practical from the perspective of attaining longitudinal static stability on (Donlan (1944), Northrop (1957), Nickel and Wohlfart (1994)).)

For an unswept wing of normal aspect ratio, the NP is located at around quarter-chord. However, as the aspect ratio is reduced to very low values, Jones (1941) states that the NP moves ahead and upward, making the attainment of stability and balance more difficult. With the majority of the payload accommodated toward the centreline of the aircraft, an increase in positive static margin will result with increased sweepback, producing favourable conditions for longitudinal static stability (Nickel and Wohlfart (1994)). The increased effective lever arm gives good trim characteristics; however, although the moment produced for 


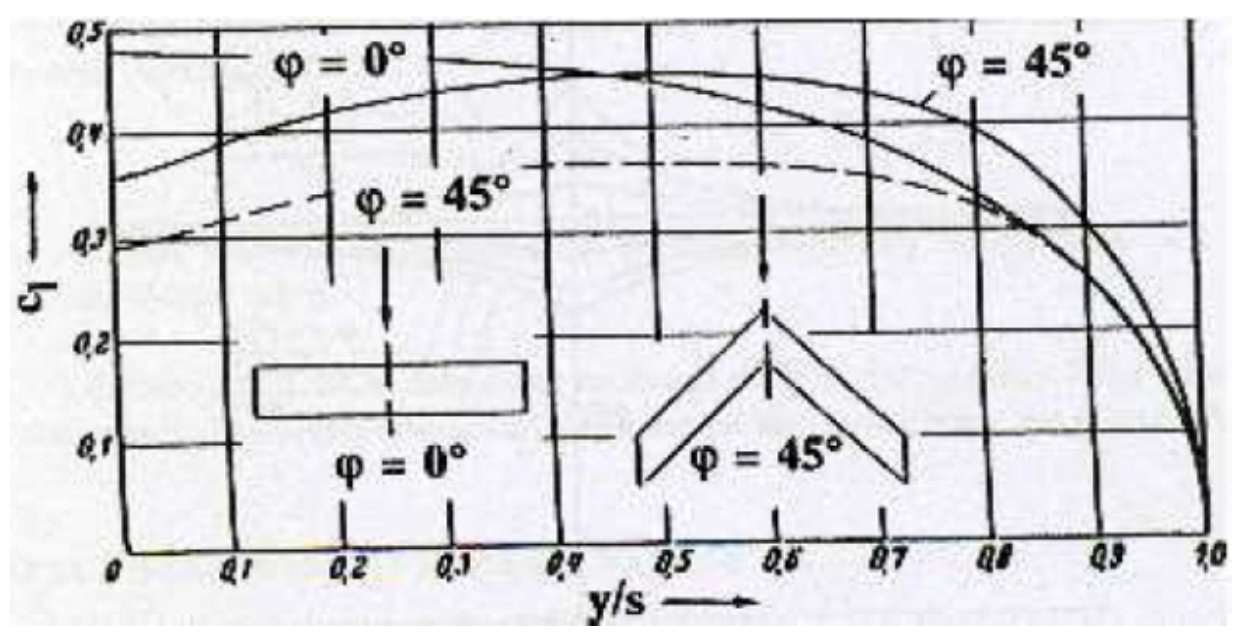

Figure 2.19: The lift distributions of two rectangular wings with an aspect ratio of 5 and sweep angles of $\phi=0^{\circ}$ and $\phi=45^{\circ}$. The lift distribution of the swept wing has been plotted for equal lift (solid line) and for the same angle of attack (dashed line). (Taken from Nickel and Wohlfart (1994)).

a given control surface deflection increases with sweep, the overall response of the aircraft is also affected by the increased moment of inertia about the lateral axis. Sweep also contributes to the effectiveness of wingtip fins (see below), producing more directional stability and rudder control or, alternatively, allowing them to be made smaller. There is the possibility of using centre spanwise surfaces as high-lift devices, whereby lift increments produce only minor changes in pitching moment. Jones (1941) explains that sweepback increases the effective dihedral, and that this effect is strongly dependent on angle of attack (increasing with lift coefficient). He adds that the lateral stability and control characteristics of tailless aircraft should be able to approach those of conventional aircraft, and concludes that weathercock stability can be secured provided sweepback is combined with either negative dihedral or wingtip fins. Sweepback helps prevent the occurrence of $\alpha$-oscillations (also known as pecking) - an oscillatory motion about the lateral axis — which can become problematic in gusty weather conditions (Donlan (1944)). Although tumbling has been shown not to be particularly troublesome, an increased amount of sweep helps prevent its occurrence (Nickel and Wohlfart (1994)).

Swept-back wings have an inherent tendency to undergo premature tip stall, especially during high-lift flight conditions such as take-off and landing. This is a very serious condition, as the aircraft will tend to "roll-off" around the longitudinal axis, and as it is a self-amplifying process (Nickel and Wohlfart (1994)). Tip stall also has an increased tendency to arise when the aircraft is yawing. Donlan (1944) shows that it first occurs over the rear portion of the 


\section{CHAPTER 2. LITERATURE REVIEW}

wing where the control surfaces are located; hence, it is likely that aileron control will be ineffective. Furthermore, the tip stall also manifests as a pitching instability, as the loss of lift often occurs behind the CG. Ground clearance issues may therefore become of importance, necessitating dihedral (though this in turn increases the skid-roll moment). Finally, flying wings with large sweepback have a stronger tendency to Dutch roll.

\section{Taper}

Taper is the other main parameter which influences both the aerodynamic performance of the FW and its construction. One specific advantage of taper is that the volume of material decreases outboard, with the largest amount of usable spar height in the wing centre (where the highest spar bending moments occur). Therefore the spar can be built smaller overall, resulting in a reduction in aircraft weight. In addition, there is a reduction in the intensity of the span loading moving outboards. Thus the outer spar sections can be built lighter, which means a concentration of weight closer to the centreline, which in combination with sweep favours a positive static margin and increased directional stability. Strong tapering does however increase the danger of wingtip stall with higher local values of $C_{l}$ outboard.

\section{Aerofoil Section}

On tailless aircraft, a nose-down aerofoil pitching moment cannot be compensated for by a horizontal stabiliser, therefore the aerofoil section $C_{M 0}$ is important. Although sweep and washout can be used to an extent for balance, alone they are unable to counter very large pitching moments. Therefore, tailless aircraft having small, or even zero, zero-lift pitching moments are preferable. This may be obtained either with a symmetrical aerofoil or a reflexed aerofoil, whereby a convex leading edge generates most of the lift whilst the concave rear part provides stability. A disadvantage with symmetric sections is that they have their minimum drag at zero lift, therefore a lifting wing would have more drag than could be achieved with a properly cambered wing to match the cruise lift coefficient.

In general, the aerofoil section only has a slight affect on the NP (Nickel and Wohlfart (1994)). However, Jones (1941) notes that an extreme reduction in thickness toward the trailing edge can slightly shift the NP rearwards.

\section{Washout}

The required washout distribution depends on the lift coefficient at which the aircraft flies with neutral elevators. Nickel and Wohlfart (1994) explain that with a low degree of washout, the elevators are neutral at high speed but are permanently deflected at low speeds. The 


\subsection{THE FLYING WING CONFIGURATION}

opposite is true for a large degree of washout. The latter condition is less desirable as there is a drag penalty over a much longer flight time. For a fixed design $C_{L}$, static margin and low $C_{M 0}$, the required amount of washout increases with a drop in planform sweep and aspect ratio. Washout may be used to mitigate the effects of wingtip stall by reducing local $C_{l}$, but at the cost of a reduced overall $C_{L}$. Nickel and Wohlfart (1994) add that the NP position is also independent of washout.

\section{Wingtip Fins}

Nickel and Wohlfart (1994) explain that the overall influence of wingtip fins on the local lift density is a reduction over the wing centre, and an increase towards the tips of the basic wing. This effect: shifts the neutral point of a sweptback wing further aft, increasing the longitudinal static margin; improves aileron effectiveness; and contributes to a reduction in induced drag below the 'optimum' elliptic case for a plane wing — also noted by Lee (1961); gives the wing an effective dihedral by generating a skid-roll moment; finally, helps reduce, even eliminate, unwanted adverse yaw effects, due to the strong vortex generated by an aileron deflection which combines with the wingtip fin to produce an opposing side force.

As the lift density is approximately equal on both sides of the wing bend, Nickel and Wohlfart (1994) suggest that it is appropriate to give the wingtip fins the exact same aerofoil shape installed at the tips of the basic wing. However, there does not seem to be an optimum wingtip fin planform, i.e. sweep, taper and aspect ratio.

\subsubsection{Flying and Handling Qualities}

The flying and handling qualities of an aircraft describe the ease and effectiveness with which it responds to pilot commands in the execution of some flight task. Cook (1997) explains that the pilot's perception of flying qualities is considered to comprise a qualitative description of how well the aeroplane carries out the commanded task; whilst handling qualities describe the adequacy of the short term dynamic response to controls in the execution of the flight task. The quantification of flying and handling qualities directly is difficult; nevertheless, quantifiable parameters include: the stability, control and dynamic characteristics of the airframe; flight control system dynamics; and response to atmospheric disturbances.

\subsubsection{Civil and Military Specifications}

With the significant advances in flight control systems since fly-by-wire technology was first used on the A320 aircraft, excellent flying qualities are now possible over all flight phases (Cook (1997)). The primary purpose of current civil aviation requirements, such as the 
European JAR-25 ${ }^{1}$ and the North American FAR-25, is to ensure safety of operation covering all aspects of aircraft design; specific requirements relating to stability, control and handling are relatively less detailed. Moreover, these specifications have been compiled for conventional aircraft with limited stability augmentation. In contrast, military specifications, the main one being MIL-F-8785C, do quantify requirements in terms of stability and control parameters. On this issue, Cook (1997) comments that an aeroplane designed to meet the military flying and handling requirements would undoubtedly also meet the civil requirements.

Since the publication of MIL-F-8785C, new technologies developed for flight control systems have enabled the exploitation of capabilities such as relaxed static stability, manoeuvre load alleviation, structural mode suppression and gust load alleviation. A more recent military specification, MIL-STD-1787B, was issued to take into account the use of high gain and high authority augmentation (Cook (1997)).

\subsubsection{Behaviour of Tailless Aircraft}

Northrop (1957) explains that the response to an abrupt vertical gust is the only form of longitudinal motion where significant differences between tailed and tailless aircraft arise. He attributes the reduced accelerations experienced by the FW to a relatively larger wing chord, increasing the time for the transient lift to build up, and a shorter effective tail length, decreasing the time interval between the disturbing impulse at the lift surface and the correcting impulse at the effective tail, so that the aeroplane tends to pitch into the gust.

With regard to the dynamic lateral response, Northrop (1957) observed that, due to the reduced wing loading of tailless aircraft and the added effect of a high effective dihedral and low weathercock stability, flight in turbulent air is somewhat rougher than conventional aircraft of similar weight. Northrop (1957) comments that although weathercock stability has a slight effect on damping rates, it reduces the amplitude of response to gusts materially. He continues that increasing the side-force derivative $C_{Y_{\beta}}$ improves Dutch roll damping very materially but has virtually no effect on the amplitude of response to gusts.

\subsubsection{Aeroelastic Behaviour}

The aeroelastic behaviour of aircraft is characterised by flutter, which manifests itself as a periodic movement of the aircraft structure in response to the interaction of the structure with the (steady) airflow during flight. Flutter is a potentially destructive phenomenon because, once excited, it grows in amplitude inducing large mechanical stresses, which can often result

\footnotetext{
${ }^{1}$ Joint Aviation Requirements - developed through the cooperation of a group of European countries. http://www.mp.haw-hamburg.de/pers/Scholz/vorschriften/JAR-25.html
} 


\subsection{LAMINAR-FLYING-WING DESIGN CONCEPTS}

in the break-up of an aircraft. It cannot be easily suppressed by a pilot control input, as oscillations typically occur at frequencies above $1 \mathrm{~Hz}$ (Nickel and Wohlfart (1994)).

For flutter to arise during subsonic flight there needs to be a coupling between at least two degrees of freedom, for example, spar-bending and wing torsion. Both tailed and tailless aircraft have many degrees of freedom, which means that both types are prone to flutter. However, with the absence of a tailplane, Nickel and Wohlfart (1994) comment that tailless aircraft may actually be slightly more flutter resistant. In fact, in the early FW aircraft flight tests by Northrop, Northrop (1957) does not comment on any aeroelastically induced problems. However, Weishaar and Ashley (1973) suggest that the main reason for this may be due to the conservative structural design of the early FW's.

Weishaar and Ashley (1973) carried out elementary aeroelastic studies to compare the behaviour of $\mathrm{FW}$ arrangements relative to conventional aircraft. In the idealised analysis, the FW was treated as a free-free beam-rod, with the conventional aircraft modelled as an equally stiff and massive cantilever beam-rod of half the span. On comparing these two simple models, in the absence of bending and torsion coupling, the analysis showed equal low torsional frequencies and pairs of higher harmonics. The analysis therefore suggests that it is unlikely for any new aeroelastic phenomena to present themselves with the FW layout. Moreover, the overall conclusion after assessing the limitations of the various models is that flutter effects for FW aircraft seem more favourable.

As the lift and inertial distributions along the span of a tailless aircraft are approximately proportional to one another, Weishaar and Ashley (1973) expect the contribution to the overall internal structural load from payload and fuel-load, e.g. during a pull-up manoeuvre or gust encounter, to be mild. Therefore, on consideration of stiffness and aeroelasticity effects, Weishaar and Ashley (1973) expect that a higher structural weight fraction will result in order to attain acceptable flutter margins, compared to that estimated from static structural design loads alone.

\subsection{Laminar-Flying-Wing Design Concepts}

\subsubsection{Greener by Design}

In arriving at the performance estimates for a LFW, Green (2002) bases his evaluation on a number of parameters from the detailed conceptual BWB study of Bolsunovsky et al. (2001). However, attempts are incorporated to allow for differences between the two configurations.

The additional weight and volume requirement of an integrated suction system is thought to favour a lower aspect ratio wing, and is consequently set at a value of 5 . With a more 
integrated flying wing, it is argued that the vortex induced drag factor should be lower, and closer to unity, therefore a value of 1.1 is assumed. The profile drag coefficient is taken as a quarter of that estimated for the the BWB, $C_{D 0}=0.0026$ - Green (2002) deems this value to be conservative based on the F-94A flight test results (see Sec. 2.1.1).

An overall propulsive efficiency typical of modern high bypass ratio turbofan engines is assumed at 37\%. Green (2002) proposes that if the cruise Mach number is dropped from 0.85 to 0.80 , and an unducted fan is utilised, an overall engine efficiency of $42 \%$ is achievable.

The LFW is taken to have a takeoff weight and span equal to that for the BWB. However, the wing loading is adjusted to account for a reduced aspect ratio and lower optimum cruise altitude.

The aircraft's mission fuel is estimated using empirical correlations based on conventional aircraft, but modified to incorporate an increase of $7.5 \%$ takeoff weight to allow for suction hardware installation (based on an empirical correlation provided by Gregory (1961)), and a $10 \%$ reduction in structural weight associated with payload; however, potential weight savings with a lower aspect ratio are not accounted for.

At 2001 technology levels, Green (2002) estimates, for a LFW utilising turbofan technology, a reduction of around $50 \%$ on a baseline turbulent aircraft. Utilising UDF engines, this figure is projected to increase to around 60\%. Allowing for 2050 technology improvements, this value further increases to around $70 \%$.

\subsubsection{Delta-Wing Aircraft with Suction}

Denning et al. (1997) argue that an integrated delta-wing aircraft, with aspect ratio in the range $3-4$, benefits from a low structural weight that can be traded off against a lower $L / D$. They add that it also permits a higher wing sweep, and hence a larger chord length for a given wing thickness (i.e. lower thickness-to-chord ratio), which then enables higher critical Mach numbers, better aeroelastic performance, and larger permissible internal volumes.

Denning et al. (1997) draw upon Lanchester's minimum drag condition to show that: $C_{L} \propto \sqrt{C_{D 0}}$. This expression implies that a reduction in profile drag consequently leads to a reduction in cruise altitude and higher critical Mach numbers via a reduced $C_{L}$; nevertheless, the aerodynamic efficiency will increase as $L / D \propto \sqrt{1 / C_{D 0}}$. In contrast, Denning et al. (1997) comment that the current aircraft design philosophy is to decrease induced drag by increasing aspect ratio as this permits higher values of $C_{L}$, requiring an increase in optimum cruise altitude and higher speeds for a given Mach number.

For a fixed aircraft geometry, and assuming: a constant cruise Mach number, optimum L/D, cruise-climb-datum cruise altitude, at fixed wing loading, of 40,000 ft, Denning et al. 
(1997) investigate the effect of an arbitrary profile drag reduction on altitude and nondimensional engine thrust $F_{N} / \delta$ (where $\delta$ is the ambient static pressure relative to that at sea level). They found that a $50 \%$ reduction in profile drag gives a $41 \%$ increase in $L / D$, an increase in ambient pressure by $41 \%$ (i.e. a reduction in cruise altitude to $33,000 \mathrm{ft}$ ), and a 50\% reduction in non-dimensional engine thrust requirement. Denning et al. (1997) also document the impact that additional suction penalties, in terms of the suction coefficient, have on range performance. In Fig. 2.20 we see that the overall range performance is rather insensitive to the suction assumptions.

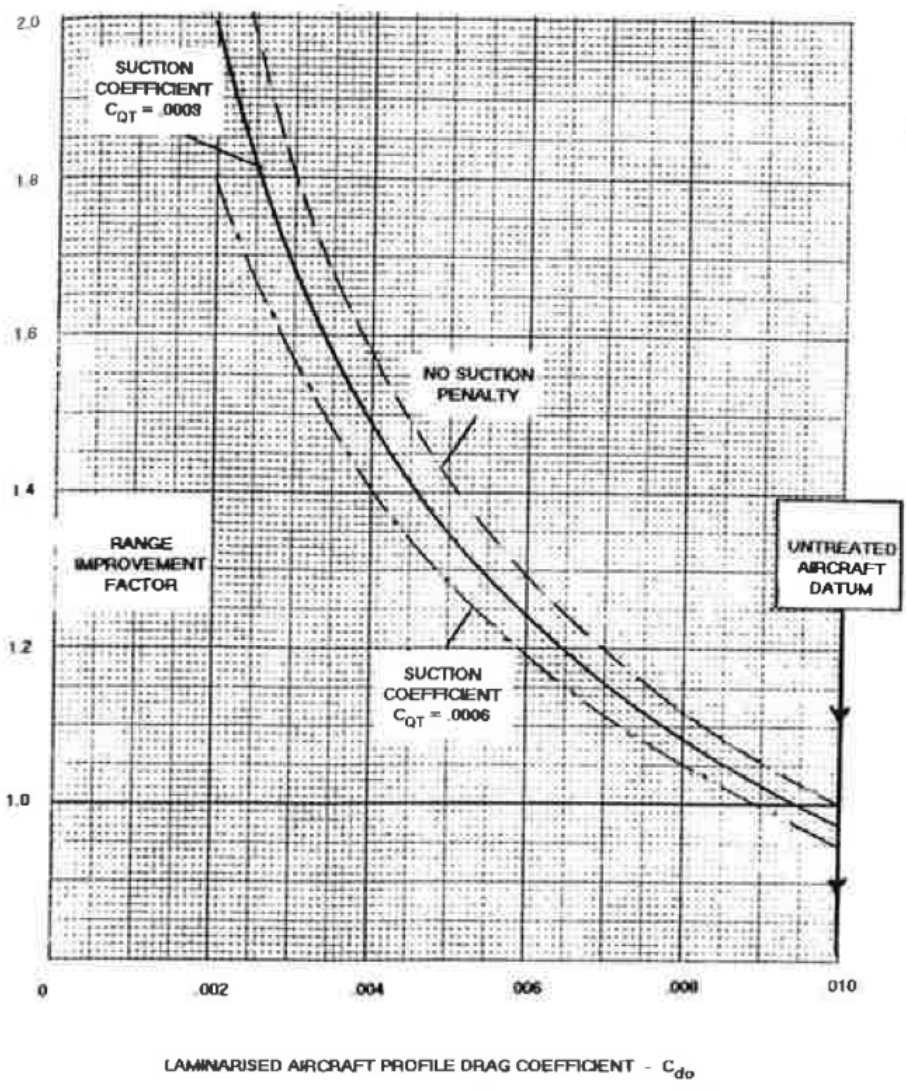

Figure 2.20: Laminarised aircraft - relative range performance - fixed integrated delta planforms. Assumptions: optimum $L / D$ cruise (no allowances), cruise Mach number of 0.8, datum $C_{D 0}=0.010$ (no suction treatment), and $C_{Q}=0.0005$. (Taken from Denning et al. (1997)). 


\subsubsection{The Handley Page HP117 Proposal}

The basic design philosophy of the HP117 proposal, documented by Lee (1961), is focussed on the minimisation of direct operating costs. This parameter acts as a performance index for both transport efficiency and first cost. Economy of operation is achieved through the combination of LFC with the all-wing arrangement and a high cruise speed. However, some loss in aerodynamic/engine efficiency is accepted to reduce design/development expenses.

Four constraints are imposed on the design: 1) low cruise drag, 2) large internal volume, 3) high critical Mach number, and 4) good low-speed behaviour. The mission specification consists of: transatlantic flight at a cruise Mach number of 0.8 , with a payload of 300 passengers and $4500 \mathrm{~kg}$ of freight. (Lee (1961) comments that the true benefits of the LFW can only be realised with over 200 passengers and a range greater than $2000 \mathrm{~nm}$.) The proposed configuration is shown in Fig. 2.21, and the design specification is summarised in Tab. 2.2.

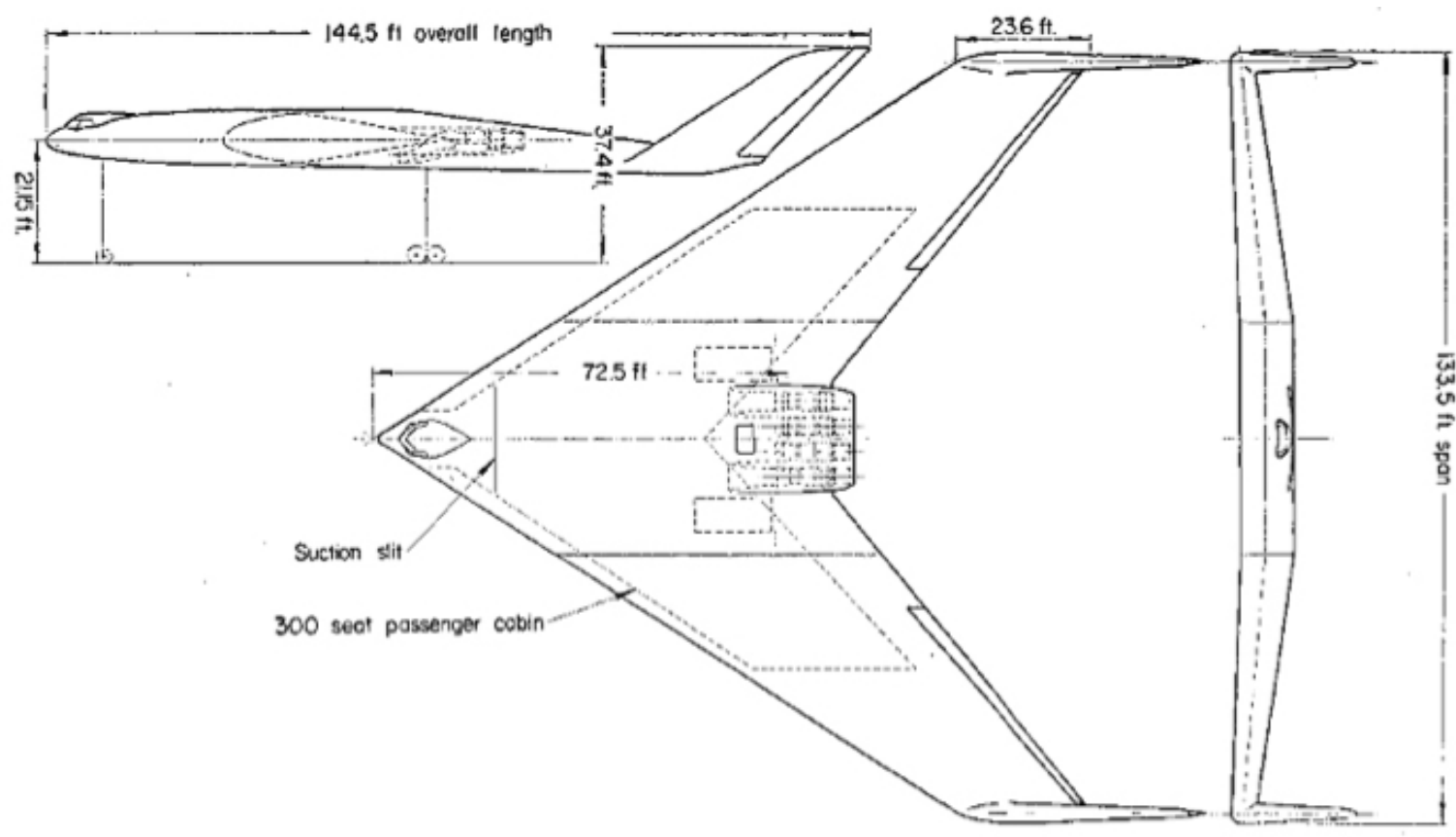

Figure 2.21: Handley Page HP117 LFW configuration (from Green (2006)).

The design features a high degree of sweepback from the perspective of yielding a reasonable value for thickness-to-chord ratio to permit satisfactory passenger accommodation, whilst, combined with a low cruise $C_{L}$, allowing for a high cruise Mach number; however, Lee (1961) highlights that this is limited to $50^{\circ}$ for fear of tip stall issues, and problems with the undercarriage design and adverse lateral stability characteristics. 
For a given payload, and hence cabin geometry, the wing area is minimised to reduce structural weight. It is argued that smaller wing areas also have the added benefit of higher wing loadings, lower cruise altitudes and, consequently, smaller engines. However, the upper value for wing loading is limited by low speed performance, unit Reynolds number, and the desire for a high critical Mach number.

Taper ratio is selected on the basis of passenger accommodation volume. However, the need for wingtip fins, which provide the necessary directional control, prevents the adoption of a triangular planform, whilst structural efficiency and passenger comfort considerations limit the feasibility of an untapered planform. The final taper ratio leads to a cabin volume $10-15 \%$ lower volume than the maximum possible.

Cabin volume reduces with aspect ratio; however, direct operating costs, assessed on the basis of structural and powerplant considerations, also reduce with aspect ratio. Therefore, a compromised aspect ratio is selected.

Table 2.2: HP117 aircraft design specification (from Lee (1961)).

\begin{tabular}{lc}
\hline \hline Maximum thickness $(\mathrm{m})$ & 2.9 \\
Root thickness-to-chord ratio & 0.13 \\
Outboard thickness-to-chord ratio & 0.20 \\
Tip thickness-to-chord ratio & 0.15 \\
Span $(\mathrm{m})$ & 40 \\
Unit Reynolds number $\left(\mathrm{m}^{-1}\right)$ & $7.5 \times 10^{6}$ \\
Sweep (degrees) & 50 \\
Mean chord $(\mathrm{m})$ & 14.5 \\
Planform area $\left(\mathrm{m}^{2}\right)$ & 581 \\
Aspect ratio & 2.7 \\
Taper ratio & 0.33 \\
Cruise lift coefficient & 0.17 \\
Mach number & 0.80 \\
Altitude $(\mathrm{ft})$ & 30,000 \\
Velocity $(\mathrm{m} / \mathrm{s})$ & 243 \\
Allowable weight $(\mathrm{kg})$ & $140 \times 10^{3}$ \\
Wing loading $\left(\mathrm{N} / \mathrm{m}^{2}\right)$ & 2340 \\
\hline \hline
\end{tabular}

The cabin is integrated with the outer wing structure: the upper and lower panels are supported by the front and rear spars, and ribs. The suction surface is integrated within the structure. The structural elements are sized based on load conditions, and include: the skins; the spars, including curved cabin pressure walls; and the floors. The rest of the aircraft is sized using standard weight estimation techniques available at the time for a conventional metal 
alloy. In contrast to conventional aircraft, the report suggests that ground loads would be a more critical design condition than in flight, due to the distribution of structural and payload weight across the wing span. A structural weight fraction of $27 \%$ MTOW is estimated; however, Lee (1961) comments that the cabin weight estimate may be optimistic.

A low cruise drag is achieved using slit suction: the resulting low profile drag permits a large wing area without a parasite drag penalty, and hence low span loading and induced drag. Laminarised tip fins increase the effective aspect ratio, without any costs in terms of parasite drag. Consequently, a vortex induced drag factor of 0.943 is assumed. To avoid turbulence contamination downstream of areas not available for laminarisation, such as the cockpit windshield and doors, 'clean-up' slots are utilised, which enable a new laminar boundary layer to begin downstream.

The powerplant installation consists of six (very) low bypass-ratio Rolls-Royce RB.163 engines. One group of engines receive sucked boundary layer flow, whilst another group receive air from the clean-up slots, supplemented with contributions from ram intake. This scheme was selected as it had an advantage in terms of first cost benefit over a separate suction pump system. As prefigured by Lachmann (1955), Lee (1961) identifies takeoff thrust as the most significant engine design feature.

Lee (1961) gives the overall, effective, cruise $L / D$ at 33 . With a cruise lift coefficient of 0.17 , a vortex-induced drag factor of 0.943 , and an aspect ratio of 2.7 , the induced drag coefficient $C_{D, i}$ is 0.0032 , and therefore the profile drag coefficient (including pump drag) $C_{D 0}$ is 0.0020 . Consequently, the low value for aspect ratio has compromised the aircraft's aerodynamic efficiency. On the basis of the Handley Page cruise and climb performance studies, which assume suction availability for altitudes above 15,000 ft, the estimated fuel burn of the HP117 is around $18 \mathrm{~g} /$ pax.km.

\subsection{Summary and Research Proposal}

Green (2002) projects that a UDF-powered laminar-flying-wing aircraft with 2001 technology can potentially achieve up to a $60 \%$ reduction in specific fuel burn on a baseline-turbulent aircraft, increasing up to $70 \%$ if 2050 technology levels are also factored in. Such potential clearly warrants further examination.

Unfortunately, there is little useful information in the open literature on laminar-flyingwing aircraft. Denning et al. (1997) present a largely qualitative discussion of the benefits of low-aspect-ratio, integrated delta-wing planforms, including consideration of drag reduction via $\mathrm{LFC}$, but do not define in detail an optimum aircraft layout nor provide a quantitative fuel-burn estimate. It is necessary to go back 50 years, to the Handley Page HP117 aircraft, 


\subsection{SUMMARY AND RESEARCH PROPOSAL}

documented by Lee (1961), to find a reasonably detailed conceptual design study with this information. The HP117 has an estimated specific fuel burn of around $18.0 \mathrm{~g} / \mathrm{pax} . \mathrm{km}$. This figure is only marginally better than that for the forthcoming B787 aircraft, which is forecast to consume $19.6 \mathrm{~g} /$ pax. $\mathrm{km}^{1}{ }^{1}$. In other words, although the Handley Page design would have been a major improvement on aircraft of the 1960s, it falls short of the performance suggested by Greener by Design. New studies, assuming present-day technology, are therefore required to substantiate (or challenge) the Greener by Design estimate. The overall objective of this thesis is to produce one such study.

We have seen that the basic architecture of a practical LFC suction system is well established. However, there is still little general guidance available on the important design parameters and their interactions. Furthermore, the requirements imposed on the system by a passenger-carrying LFW are likely to differ significantly from the conventional context, as revealed by the Handley Page study. As a consequence of the viscous drag reduction, the induced drag was also small, which in turn required a wing with low span loading, operating at a lift coefficient below conventional values. Finally, the freedom to optimise section geometry was restricted by passenger accommodation requirements, which in turn lead to thick aerofoil sections, and moment balance constraints. These changes therefore reduce the applicability of the past LFC studies to the configuration of interest here.

The HP117 study started from a perceived market need, which then defined a mission (i.e. range, speed, payload) and, eventually, the aircraft itself (as is normal in aircraft design). In contrast, it is more productive to reverse this approach for our LFW; specifically, start from the premise that an LFW is to be designed, with an emphasis above all on fuel efficiency, and then assess its capabilities once the design is complete. This means that the approach departs in some respects from a conventional methodology; by so doing, it ensures that the resulting LFW will not be unnecessarily constrained by current practice. With this philosophy, a high-level exploration of the design space can be performed to identify a specification for the key configuration and mission parameters of the proposed LFW. On the basis of this specification, a detailed conceptual design study can be undertaken. To quantify the direct benefits associated with a flying-wing configuration utilising LFC, it is necessary to compare against the performance of a conventional baseline-turbulent aircraft with the same mission and propulsion characteristics.

It should be acknowledged that, while LFC is proven in principle, its operational use remains subject to practical difficulties. Surface finish requirements are demanding, and environmental contamination is a major concern. Of interest here, however, is the fuel-efficiency potential of the LFW. This can be assessed independently of operational issues. Indeed, con-

\footnotetext{
${ }^{1}$ http://www.greencarcongress.com/2007/07/boeing-rolls-ou.html, January 2012
} 
cern over the latter seems unnecessary until it is clear that the LFW holds sufficient promise to justify its radical nature.

There is obvious merit in performing simpler studies ahead of more complex ones. Therefore, extensive and systematic optimisation procedures shall be eschewed in favour of reasoned design choices, and a configuration that is amenable to analysis by simplified methods shall be insisted upon. It is thus unlikely that the resulting concept aircraft will represent the best possible LFW, but it will provide a useful first indication of what may be expected of the type. Equally, any critical restrictions in the design tools will become apparent, allowing future development efforts to be efficiently targeted. 


\section{CHAPTER 3}

\section{Design Methodology}

In this chapter, an overview of the conceptual design approach for conventional aircraft is given, and compared against that envisaged for a LFW aircraft. Then, the numerical tools and design methodologies employed in this study are discussed.

\subsection{General Approach}

The conventional approach to an aircraft conceptual design problem begins with the definition of a mission specification. Then, a suitable donor aircraft is usually identified, and is used to base initial estimates of target weight, surface areas, etc. The design approach, following e.g. Raymer (1999), is then as follows:

(1) target gross weight specified;

(2) cruise $C_{L}$ estimated, thereby fixing cruise altitude;

(3) tailplane geometry defined for balance and stability;

(4) detailed drag assessment in the en-route configuration provides an estimate of the liftto-drag ratio;

(5) aircraft structural weight estimated;

(6) engine sized and fuel weight to complete mission estimated;

(7) MTOW evaluated and, if higher than initial target specification, process repeated from (2) with revised MTOW.

In contrast, with an emphasis above all on fuel efficiency, the design process for the LFW is reversed. The design methodology, shown schematically in Fig. 3.1, is broken down into five 
main parts: 1) 3-D airframe generation, 2) aircraft performance analysis, 3) suction-system technology integration, 4) propulsion system design, and 5) structures and weights. (However, as is evident from the figure, the design process is not linear.) Once a viable aircraft has been identified, the mission can be specified. For each design area, it is helpful to consider what are the main inputs and outputs, what are the constraints, and what must initially be assumed. These shall be discussed further as the conceptual design evolves in Chaps. 5 and 6. Before this, an overview of the technical approach is provided in the following section; note, the suction-system design approach is presented in Chap. 4.

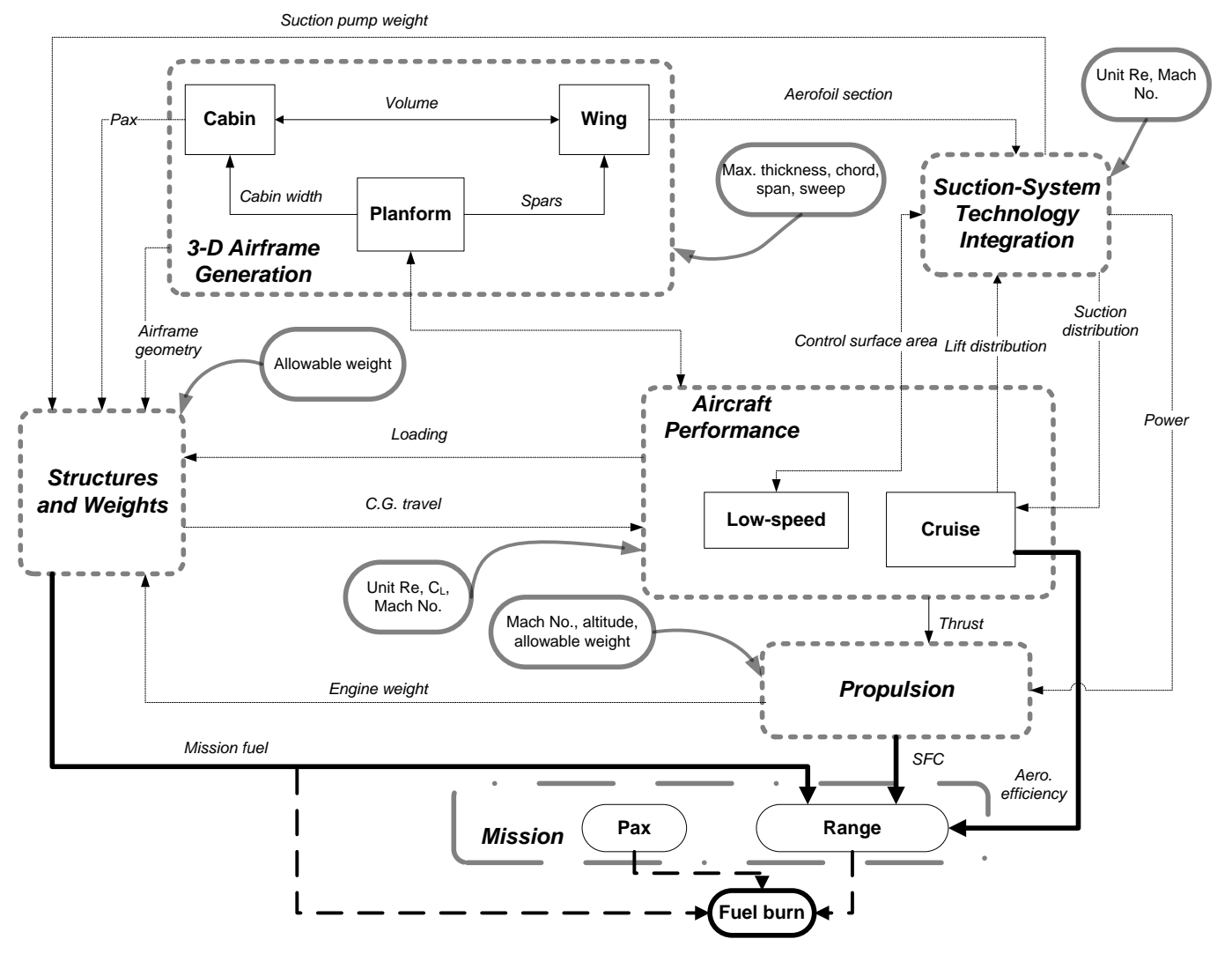

Figure 3.1: Schematic diagram of the design process envisaged for the LFW. 


\subsection{Technical Approach}

\subsubsection{3-D Airframe Generation and Aircraft Performance}

A quasi-3D approach is employed to evaluate the aircraft aerodynamics, as a full 3-D analysis is too costly to fully explore the design space. AVL $3.27^{1}$, a numerical design tool that implements a (2-D) vortex lattice method, is used to evaluate the spanwise lift distribution, aircraft stability derivatives and induced drag coefficient. This is combined with a sectional inviscid aerofoil analysis in VGK (Ashill et al. (1987)) to evaluate the surface pressure distribution. (Note, a viscous analysis is performed in XFoil to check for flow separation on evaluation of control surface deflections.) The Eppler and Somers (1980) boundary-layer solver is then used in conjunction with a suction-system design algorithm, developed in Chap. 4, to evaluate the suction requirements for the maintenance of laminar flow, and the corresponding section viscous drag coefficients with and without suction. Additional aircraft drag contributions are accounted for using empirical correlations in Torenbeek (1976) and Raymer (1999).

\subsubsection{Computational Tools}

\section{AVL}

AVL implements a vortex-lattice model suited for aerodynamic configurations mainly consisting of thin lifting surfaces at small angles of attack and sideslip. Furthermore, it assumes quasi-steady flow, and therefore neglects the shedding of unsteady vorticity; consequently, the period of oscillatory motions should be much longer than the time it takes for the flow to traverse the wing chord.

To better represent the characteristics of thick aerofoils, the lift-curve is scaled by $C_{L \alpha}$, which from $2 \mathrm{D}$ potential flow theory, is

$$
C_{L \alpha}=1+0.77 \frac{t}{c}
$$

The effect is a chordwise shift of the control point relative to the bound vortex on each element. However, the AVL user-primer notes that viscous effects will reduce the 0.77 factor. Finally, slender bodies, such as fuselages, are modelled as lines of distributed source/sink elements.

The mathematical analysis of transonic flow regimes, where there are regions of subsonic and supersonic flow, involves the solution of a set of non-linear differential equations that require advanced computational techniques. However, assuming small perturbations to the

\footnotetext{
${ }^{1}$ http://web.mit.edu/drela/Public/web/avl/
} 


\section{CHAPTER 3. DESIGN METHODOLOGY}

(inviscid) free-stream flow conditions, linearised theories can be applied. AVL employs one such method: the classical Prandtl-Glauert equation of motion in the compressible plane is transformed to the Laplace equation, which is then solved by incompressible methods (see, e.g., Houghton and Carpenter (1993) for further details). The forces are then computed by applying the Kutta-Joukowsky relation to each vortex, which remains valid for compressible flow.

The AVL user-primer details that surfaces and wakes are represented as a single-layer of vortex sheets, which are discretised into horseshoe vortex filaments whose trailing legs are parallel to the x-axis (as depicted in Fig. 3.3). By continuity, the streamlines downstream of an aircraft fuselage, and hence wake, contract; this is depicted in Fig. 3.2, whereby the annular streamtubes are mapped to a different spanwise position in the Trefftz Plane. Similarly, the wing annular streamtube contracts to another annular streamtube in the wake, but with the same cross-sectional area. (Due to fuselage viscous-wake effects, Greitzer et al. (2010) explain that the centre wake radius is taken to be non-zero and the circulation in this region is not evaluated; therefore, as highlighted in the AVL user-primer, the approach is only valid for cases where the fuselage is expected to have a negligible influence.)
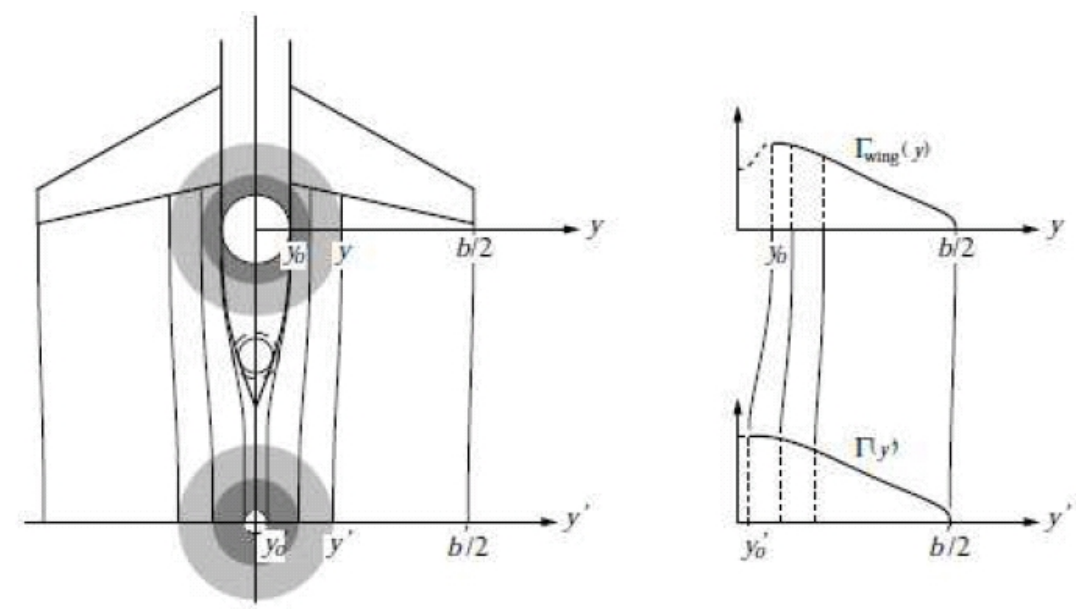

Figure 3.2: Wake streamline contraction due to fuselage thickness, carrying wing circulation into the wake (from Greitzer et al. (2010)).

The perturbation velocities in the horizontal and vertical directions in the Trefftz Plane are calculated from the circulation distribution immediately behind the trailing edge which is convected into the wake. These are used to evaluate the overall lift and induced drag.

A resolution study was performed to investigate the necessary balance between the desired accuracy levels and computational efficiency. It revealed that this balance is achieved with 20 
chordwise and 40 spanwise vortices, with a distribution such that a higher density of panels are concentrated toward the tips, as shown in Fig. 3.3.

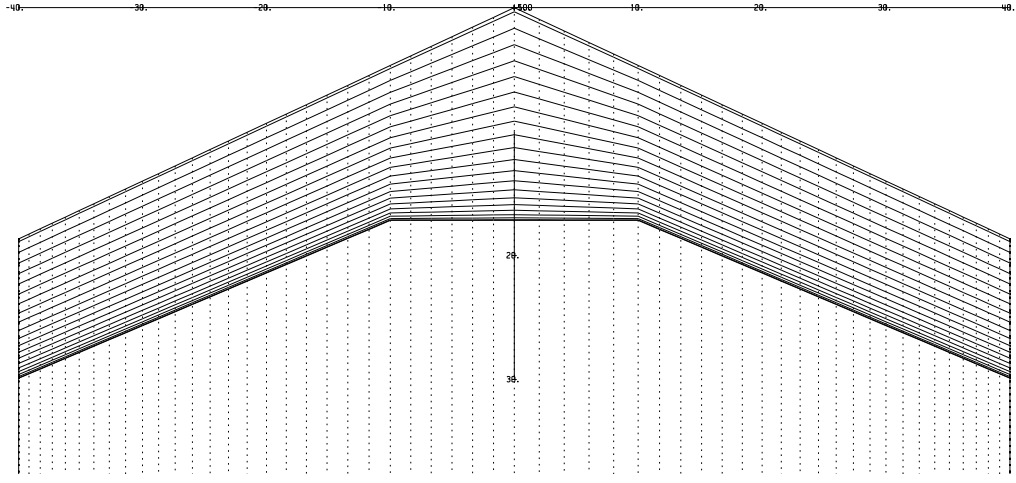

Figure 3.3: Example distribution of bound and trailing vortices (output from AVL).

\section{XFoil}

XFoil implements a linear-vorticity streamfunction formulation that solves the incompressible potential flow equations. The aerofoil contour is discretised into flat panels, with the twodimensional inviscid flowfield constructed by superposition of a free-stream flow, a vortex sheet on the aerofoil surface, and a source sheet on the surface and in the wake. A panel of uniform source and vortex strength is placed across the trailing-edge if there is a finite thickness. The vortex sheet strengths are determined by invoking the Kutta condition and requiring that the streamfunction at each node on the surface be equal to a constant. For viscous flows, the source strengths are determined by invoking the boundary-layer momentum and energy integral equations. The $e^{N}$ method is employed to determine the transition location. Much like AVL, XFoil uses a correction to determine the surface $C_{p}$ on an aerofoil in compressible flow: the Karman-Tsien formulation. Further details can be found in Drela (1989).

\section{VGK}

VGK (Viscous Garabedian-Korn) developed by Qinetiq, formerly RAE, is a two-dimensional viscous-inviscid interaction type solver. In contrast to AVL and XFoil, VGK solves the full steady potential flow equations for a compressible gas. These equations are exact in the case of shock-free flow. The potential equations are solved on a mesh by means of an iterative finite difference scheme with a relaxation method. A conformal mapping function generates the 


\section{CHAPTER 3. DESIGN METHODOLOGY}

mesh in the external region of the aerofoil. First, a small number of iterations are performed on a coarse mesh to speed up the procedure, then a large number of iterations are performed using a fine mesh to achieve a converged and accurate solution. With the Eppler and Somers (1980) method employed in this study (see Chap. 4), the reader is referred to ESDU 96028 (2004) for further details of the viscous solver, and drag calculation methods utilised by VGK.

\subsubsection{Aircraft Profile Drag Estimation Method}

Torenbeek (1976) explains that aircraft profile drag can be subdivided into five groups: wing, fin and stabiliser; fuselage; engine nacelles; viscous interference; and protuberances, surface imperfections, gaps, etc. The methods used to determine these contributions are presented in this section.

\section{Wing Profile Drag}

Determination of the drag coefficient for a swept wing requires careful consideration. In Fig. 3.4 a schematic diagram of an infinite swept wing, with the direction of the potential flow streamline detailed, along with the pressure and friction drags, is provided. The friction drag is shown to act mostly in the free-stream direction, whilst pressure drag, which in shock-free flow is due to the viscous displacement of the boundary layer, acts normal to the wing-spanwise axis (i.e. normal to the isobars, which for quasi 2-D flow are aligned parallel to the wing-spanwise axis).

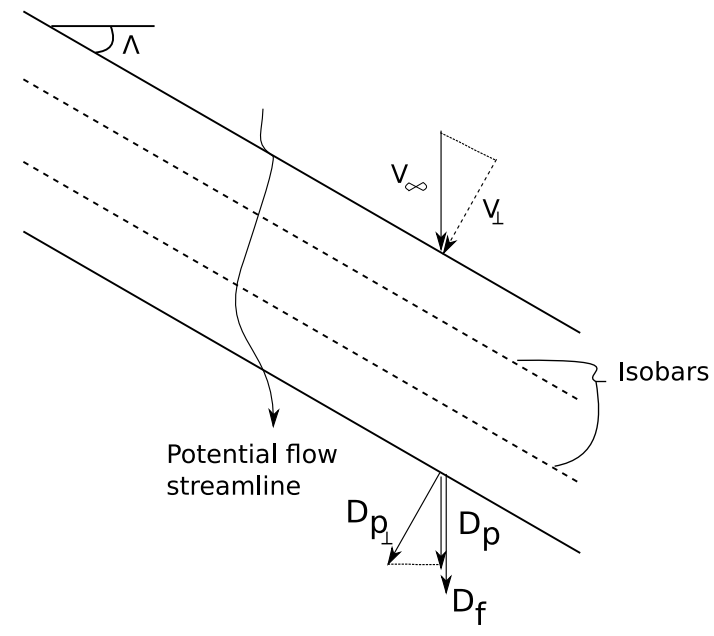

Figure 3.4: Schematic diagram of friction and pressure drag forces on an infinite swept-wing (Greitzer et al. (2010)). 
In principle, the friction and pressure drag have to be calculated independently, but this is not straightforward via purely 2-D calculations. Two noteworthy approaches have been developed by Ashill et al. (1988) and Greitzer et al. (2010), and are discussed here.

Ashill et al. (1988) first take the perpendicular-plane pressure drag and resolve it into the free-stream direction. This is then combined with the skin-friction drag. Hence,

$$
C_{D_{v}}=C_{D_{p_{\perp}}} \cos ^{3} \Lambda+C_{D_{f}}
$$

where $C_{D_{f}}=\frac{D_{f}}{1 / 2 \rho_{\infty} U_{\infty}^{2} c}$. The skin friction drag is also calculated in the wing-normal direction, using an equivalent Reynolds number. It is then assumed that the flow in a turbulent boundary layer is aligned parallel to the free stream, provided the wing is sheared; they conclude then that, the skin-friction drag coefficient is independent of sweep angle

$$
C_{D_{f}}(R e, \Lambda)=C_{D_{f}}(R e, 0) .
$$

(A power law for turbulent flows is used to allow for Reynolds scaling effects.)

Greitzer et al. (2010) adopt a similar approach. The skin-friction and pressure drag coefficients are also calculated in the perpendicular plane, but are then scaled by a "Rescaling exponent", appropriate for a fully turbulent flow, to obtain $C_{D_{f}}$ and $C_{D_{p}}$. It is then assumed that $C_{D_{f}}$ acts mostly in the free-stream direction and scales with the dynamic pressure in this direction, whilst the pressure drag scales with the wing-normal dynamic pressure and acts in this direction.

Both methods are approximate as the potential-flow streamline is not aligned in either the wing-normal or streamwise direction across the entire chord length, so the use of onedimensional integral equations for the boundary layer introduces errors. Therefore, the skin friction drag estimate is strictly not correct; in contrast, the perpendicular-plane pressure drag estimate is correct, if determined by integrating the surface pressure distribution normal to the isobars. Moreover, both methods rely on a Reynolds number scaling applicable to turbulent boundary layers. Furthermore, the infinite swept-wing model breaks down near the wing root, where the potential flow streamline is necessarily aligned in the free-stream direction.

The approach employed in this study is to estimate the profile drag coefficient from a boundary-layer calculation in the free-stream direction, assuming that the error in the pressure drag term is negligible. The surface velocity distribution in the normal plane, $u_{\perp}$, is evaluated through an inviscid calculation using, for example, XFoil or VGK. The external velocity 'seen' by the boundary layer consists of the $u_{\perp}$ component and the component due 
to sweep, $U_{\infty} \sin \Lambda$. When resolved and added, they give a streamwise external velocity

$$
u_{e}=U_{\infty} \sin ^{2} \Lambda+u_{\perp} \cos \Lambda
$$

This external flow velocity is then used as an input to the boundary-layer calculation described in Chap. 4. For a sharp trailing edge aerofoil, the Squire-Young formulation (Young (1989)), which is based on a 'far-field' approach for shock-free flow, is then used to evaluate the viscous drag coefficient,

$$
C_{D_{v}}=2 \frac{\delta_{2, \infty}}{c}=2 \delta_{t e}\left(\frac{u_{e}}{U_{\infty}}\right)^{\frac{H_{12}^{t e}+5}{2}}
$$

\section{Component Profile Drag Contributions}

Torenbeek (1976) explains that in pre-design applications, where the aeroplane shape is not fully defined, the 'flat plate analogy' may be used. This method assumes that the component shapes can be treated as smooth streamline bodies, and therefore the body profile drag can be related to that of a flat plate. The skin-friction drag coefficient of a flat plate, including Mach number effects (Eq. 12.27, pp. 282 in Raymer (1999)), is

$$
C_{F}=\frac{0.455}{\left(\log _{10} R e\right)^{2.58}\left(1+0.144 M^{2}\right)^{0.65}},
$$

assuming transition at the leading edge.

This is related to the profile drag of the object through a shape correction factor $\phi_{f}$ via (Eq. F-27, pp. 498 in Torenbeek (1976))

$$
C_{D_{0_{b a s i c}}}=C_{F}\left(1+\phi_{f}\right) \frac{S_{w e t}}{S_{r e f}},
$$

which accounts for the difference in skin friction due to different boundary-layer flow development around an object, variation in boundary-layer edge velocity due to the object's thickness, and pressure drag.

\section{Fuselage}

For a streamlined fuselage (without a blunt nose or short-afterbody tail section), the fuselage drag consists of a basic contribution (corresponding to an uncambered, smooth, streamline body at zero incidence, and no jet efflux), and a correction for angle of attack and up-sweep 
effects (Eq. F-37 pp. 502 in Torenbeek (1976))

$$
C_{D_{0_{f}}}=C_{D_{0_{\text {basic }}}}+\Delta_{\alpha \beta} C_{D_{0_{f}}}
$$

The basic drag contribution is evaluated using Eqs. 3.6 and 3.7. The Reynolds number is based on the fuselage length $l_{f}$. The shape factor $\left(\phi_{f}\right)$ is evaluated using Eq. 3.9 (Eq. F-41, pp. 502 in Torenbeek (1976)), which is a function of the slenderness ratio $\left(\lambda_{e f f}\right)$ given by Eq. 3.10 (Eq. F-40, pp.502 in Torenbeek (1976))

$$
\begin{gathered}
\phi_{f}=\frac{2.2}{\lambda_{e f f}^{1.5}}+\frac{3.8}{\lambda_{e f f}^{3}} \quad \text { for } \quad \frac{l_{A}}{D_{f_{e f f}}} \geq 2 . \\
\lambda_{e f f}=\frac{l_{f}}{D_{f_{e f f}}} \quad \text { or } \quad \lambda_{e f f}=\frac{l_{N}+l_{A}}{D_{f_{e f f}}}+2
\end{gathered}
$$

whichever is less; $D_{f_{e f f}}=\sqrt{\frac{4}{\pi} A_{c}}$, where $A_{c}$ is the (mean) fuselage cross-sectional area; $l_{N}$ and $l_{A}$ denote the length of the nose and afterbody regions, respectively.

According to Torenbeek (1976), a reasonable approximation of the fuselage profile drag increment due to angle of attack and fuselage upsweep can be made on the basis of the 'cross-flow concept'. The drag on an element, as shown in Fig. 3.5, with a circular cross section (equal to the local fuselage cross section) perpendicular to the airflow, for an isolated, infinitely long, cylindrical fuselage in uniform flow, is integrated along the length of the fuselage. The result is plotted in Fig. 3.6 for typical values of slenderness ratio and ratio of planform areas in sections I and II. For fuselage incidence, $\alpha_{f}$, and upsweep angle, $\beta$, values in excess of $5^{\circ}$, the extra drag starts to become a significant proportion of the basic drag.

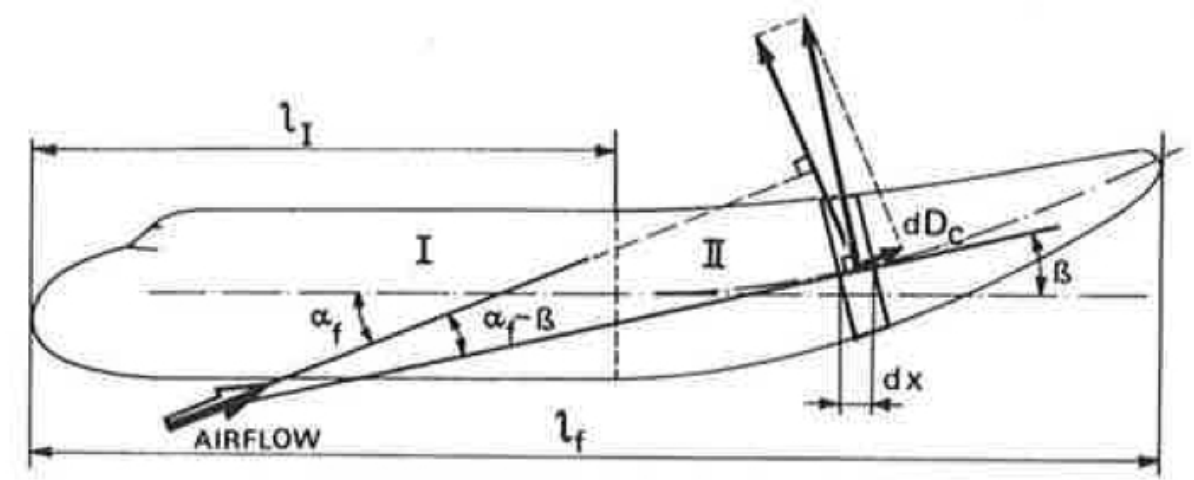

Figure 3.5: Derivation of fuselage cross-flow drag (from Torenbeek (1976)). 


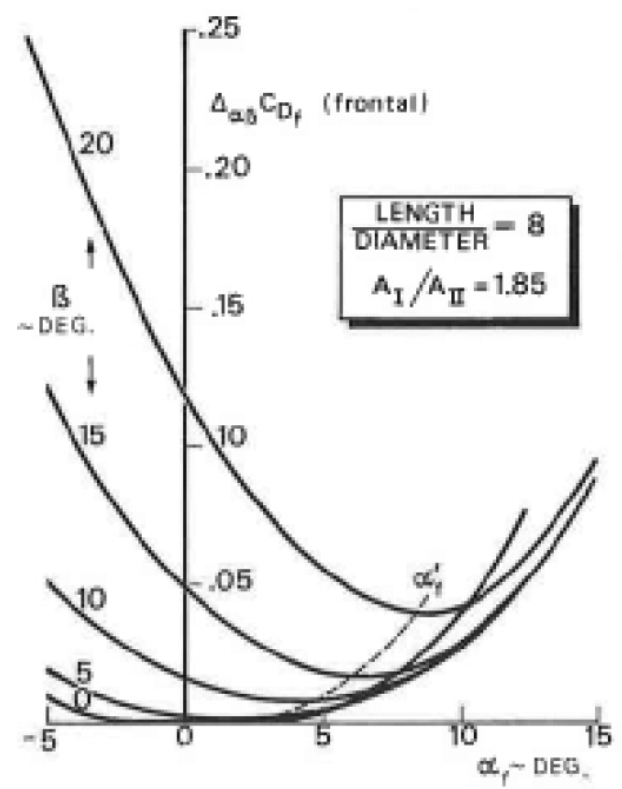

Figure 3.6: Estimated fuselage profile drag increment due to angle of attack and rear fuselage upsweep (from Torenbeek (1976)). ( $\alpha_{f}^{\prime}$ demarcates the fuselage incidence at which the extra drag is a minimum for a given $\beta$ value.)

\section{Wing/Fuselage Viscous Interference}

Lift effects result in a thickening of the boundary layer flow near the wing/fuselage intersections, and an increase in local flow velocity. At small angles of attack, Torenbeek (1976) (Eq. F-66, pp. 509) gives the drag increment to be approximately

$$
\Delta_{i}\left(C_{D} S\right)_{p}=1.5 C_{F} t_{r} C_{c i} \cos ^{2} \Lambda_{1 / 2}
$$

where: $C_{c i}$ is the total circumferential length for both wing halves of the wing/fuselage intersection line, $t_{r}$ is the thickness of the wing section at the root, $\Lambda_{1 / 2}$ is the mid-chord-line sweep, and $C_{F}$ is evaluated near the wing/body interconnection.

Torenbeek (1976) adds that the pressure gradients and section forces increase on the upper side of the wing, and decrease on the lower side. The lower side effects may be mitigated by suitable filleting, whilst, on a high-mounted wing, the fuselage drag on the upper side is reduced at high lift coefficients. A simple correction is provided by Torenbeek (1976) (Eq. F-67 pp. 510)

$$
\frac{\Delta_{i}\left(C_{D} S\right)_{p}}{C_{F} C_{L} c_{r} D_{f_{e f f}}}=-0.81
$$


where $c_{r}$ is the root-chord length.

Finally, as a consequence of the wing circulation, the cross flow effects on fuselage drag are affected. Torenbeek (1976) suggests that this form of interference may be taken into account by reducing the value for $\alpha_{f}^{\prime}$ (detailed in Fig. 3.6). However, for low values of $\beta$ and $\alpha_{f}$ the effects are negligible.

\section{Tailplane and Tailplane/Airframe Viscous Interference}

Torenbeek (1976) comments that tailplane viscous interference effects account for a small proportion of the tailplane profile drag. He adds that this it is justifiable to assume that no extra drag need be accounted for.

\section{Nacelle and Pylon}

Nacelle and pylon drag is estimated following Raymer (1999). A modified version of Eq. 3.7 is used to account for interference effects $Q$ (Eq. 12.21, pp. 281 in Raymer (1999))

$$
C_{D_{0}}=C_{F}\left(1+\phi_{c}\right) Q \frac{S_{w e t}}{S_{r e f}}
$$

where $\phi_{c}$ is the component shape factor. For components mounted directly on the wing $Q=1.5$, otherwise if they are mounted less than one engine diameter $Q=1.3$.

- The pylon shape factor (Eq. 12.30, pp. 283 in Raymer (1999)) is:

$$
\phi_{\text {pylon }}=\left[1+\frac{0.6}{(x / c)_{m}}\left(\frac{t}{c}\right)+100\left(\frac{t}{c}\right)^{4}\right]\left[1.34 M^{0.18}\left(\cos \Lambda_{m}\right)^{0.28}\right]-1,
$$

where $\Lambda_{m}$ refers to the sweep of the maximum-thickness line, and $(x / c)_{m}$ is the chordwise location of the aerofoil at the maximum thickness point.

- The nacelle shape factor (Eq. 12.32, pp. 283 in Raymer (1999)) is:

$$
\phi_{\text {nacelle }}=0.35 / f
$$

where $f$ (Eq. 12.33, pp. 283 in Raymer (1999)) is:

$$
f=\frac{l}{d}=\frac{l}{\sqrt{(4 / \pi) A_{\max }}}
$$

where $A_{\max }$ refers to the maximum cross-sectional area. 


\section{CHAPTER 3. DESIGN METHODOLOGY}

For an estimate of the drag contributions from these two components, the following geometries are assumed: a nacelle diameter of $2 \mathrm{~m}$ and length of $3.5 \mathrm{~m}$; and pylon having a chord of $3.5 \mathrm{~m}$, span of $2 \mathrm{~m}$, zero sweep, $t / c=0.1$ and maximum thickness at $x / c=0.3$. Interference effects are based on the assumption that the engines are mounted less than one diameter away.

In the event of an engine failure, not only is there a reduction in available thrust, but there is also a drag increment associated with a stopped propeller or windmilling engine. A conservative estimate of this drag contribution is provided by assuming the latter condition (Eq.12.41, pp. 290 in Raymer (1999)).

$$
C_{D_{0} \text { engine failure }}=0.3 \frac{A_{\text {engine front face }}}{S_{\text {ref }}} .
$$

\section{Miscellaneous Contributions}

The miscellaneous drag contributions considered in this study arise from the cockpit windshield, gaps where there are retracted flaps and control surfaces, and surface roughness.

\section{Cockpit Wind-Shield}

Torenbeek (1976) gives a value of 0.02 for the drag coefficient based on window frontal area. This translates to roughly $2-3 \%$ of the total fuselage drag.

\section{Gaps due to Retracted Flaps and Control Surfaces}

A drag coefficient of 0.0002 based on the projected area of the surface of interest (i.e. wing, fin and stabiliser) is assumed achievable for the control surfaces (Torenbeek (1976)).

\section{Surface Roughness}

If the size of the roughness element is less than a critical value, the surface is treated as smooth with no drag penalty. This critical roughness is given by (Eq. F-82, pp. 517 in Torenbeek (1976)):

$$
\left(\frac{k}{l}\right)_{\text {crit }}=\frac{39.5}{R e^{0.94}}
$$

where: $k$ is the equivalent sand grain roughness thickness, which Raymer (1999) gives, for smooth paint, a value of $6.3 \times 10^{-6} \mathrm{~m} ; l$ is the body or chord length; and $R e$ is based on $l$. Otherwise, the profile drag increment is evaluated using Fig. 3.7. 


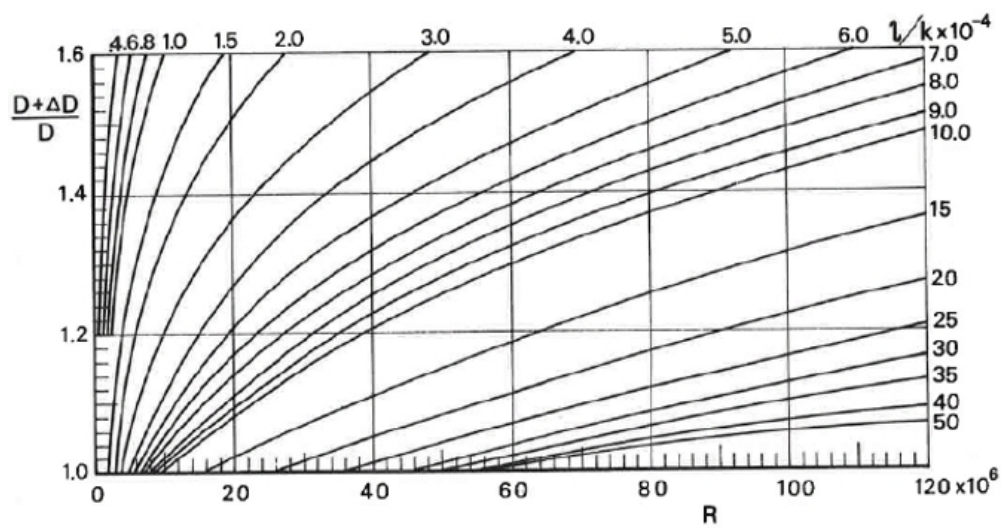

Figure 3.7: Profile drag increment due to surface roughness (from Torenbeek (1976)).

\subsubsection{Propulsion System Design}

The propulsion system design is conducted using GasTurb ${ }^{1}$ : a commercial package that performs a thermodynamic analysis to specify the engine at the chosen design point, and then assess off-design performance. To aid an understanding of the design approach adopted, an elementary description of the gas turbine provided by Cumpsty (1997) follows.

\subsubsection{Design Cycle Parameters}

The overall efficiency, which relates the work done on the aircraft to energy released from the fuel, is given by

$$
\eta_{0}=\eta_{p} \eta_{t h}
$$

The propulsive efficiency is, approximately, given by Eq. 3.20, and compares the rate of work done on the aircraft to the rate of increase of energy of the flow through the engine.

$$
\eta_{p}=\frac{2}{1+U_{j} / U_{\infty}}
$$

Whilst the thermal efficiency is given by

$$
\eta_{t h}=\frac{\dot{W}_{n e t}}{\dot{m}_{f} L C V}
$$

and relates the rate of increase of energy of the flow through the engine to the thermal energy made available by burning the fuel. Cumpsty (1997) shows that it has a functional

\footnotetext{
${ }^{1}$ http://www.gasturb.de
} 


\section{CHAPTER 3. DESIGN METHODOLOGY}

dependence on the following parameters

$$
\eta_{t h}=f n\left(T_{02}, \frac{T_{04}}{T_{02}}, r, \eta_{\text {comp }}, \eta_{t u r b}\right)
$$

where $r$ is the overall pressure ratio.

To obtain a high thermal efficiency, it is essential to operate at high values of $T_{04} / T_{02}$ and pressure ratios for the core. However, this invariably leads to a high power output, which, if used to accelerate the core flow, would lead to a high jet velocity ratio $U_{j} / U_{\infty}$ and, therefore, a poor propulsive efficiency. This may be circumvented through the utilisation of a fan or propeller, which supplies a relatively small increment in kinetic energy to a large bypass stream.

Turboprop (i.e. gas-turbine-powered propeller engines) offer the best combination of propulsive and thermal efficiency; Mair and Birdsall (1992) suggest up to a $25 \%$ lower specific fuel consumption relative to a high-bypass-ratio turbofan. However, these types of engines are limited by compressibility issues at high-subsonic flight Mach numbers.

The propeller efficiency is defined as the ratio of the useful thrust power to the shaft power:

$$
\eta_{P R}=\frac{F_{p} U_{\infty}}{P_{s}} .
$$

This is the single most important measure of propeller performance, as high efficiency is desired over all important phases of flight (Torenbeek (1976)). The thrust and power are expressed as non-dimensional coefficients

$$
C_{T}=\frac{F_{p}}{\rho_{\infty} N_{P R}^{2} D_{P R}^{4}}
$$

and

$$
C_{p}=\frac{P_{s}}{\rho_{\infty} N_{P R}^{3} D_{P R}^{5}} .
$$

These three coefficients can be related through the advance ratio

$$
\begin{aligned}
J & =\frac{U_{\infty}}{N_{P R} D_{P R}} \\
& =\eta_{P R} \frac{C_{p}}{C_{T}} .
\end{aligned}
$$

$J$ is low when the rotational speed is high relative to the flight velocity, e.g, at takeoff, whilst is higher in cruise. 
Mair and Birdsall (1992) explain that for propellers of fixed geometry

$$
\left(C_{T}, C_{P}, \eta_{P R}\right)=f n(J, R e, M) .
$$

The influence of Reynolds number may usually be taken as negligible, but the effects of Mach number are important. Neglecting the components of induced velocity, the helical tip Mach number is a function of advance ratio

$$
M_{T}=M_{\infty}\left[\left(\frac{\pi}{J}\right)^{2}+1\right]^{1 / 2}
$$

High tip Mach numbers lead to the formation of shockwaves, and rapid increases in associated drag. The same shockwaves also produce noise, and it is mainly for this reason turboprops have not been utilised by high-subsonic speed aircraft (Mair and Birdsall (1992)).

Rohrbach et al. (1982) performed wind tunnel tests on a $45^{\circ}$ swept eight-bladed propeller at free-stream Mach numbers between $0.45-0.85$. The propeller map obtained for the $M_{\infty}=0.70$ case is provided in Fig. 3.8. It details propeller efficiency contours as a function of the power coefficient and advance ratio, and the tip Mach number variation with advance ratio. Tip Mach numbers in the range $0.8-1.1$ are achieved through varying blade angle, which is also detailed in the figure. The propeller map shows that propeller efficiencies of up to $82 \%$ have been demonstrated for this case.

One final, interesting, point is discussed by Mair and Birdsall (1992). If the increase of velocity $\left(U_{j}-U_{\infty}\right)$ across a propeller of area $A$ is uniform, the propeller thrust is

$$
\begin{aligned}
F_{p} & =\frac{1}{2} \rho_{\infty} A\left(U_{j}^{2}-U_{\infty}^{2}\right) \\
\Rightarrow \frac{2 F_{p}}{1 / 2 \rho_{\infty} A U_{\infty}^{2}} & =\left(\frac{U_{j}}{U_{\infty}}\right)^{2}-1 .
\end{aligned}
$$

This tells us that propulsive efficiency, as given by Eq. 3.20, is related directly to the propeller loading $F_{p} / A$. The higher the loading, the lower the propulsive efficiency (as more power is lost to the increasing kinetic energy of the flow). Furthermore, for a constant propeller thrust, it shows that increasing diameter will lead to better efficiency.

Raymer (1999) points out that the limitation on this is the propeller tip speed - large diameter blades require low rotational speeds to avoid compressibility issues, as discussed earlier. However, the propeller is driven by a low pressure turbine, which generally operate at high rotational speeds (as the power output is proportional to the square of the blade speed). Therefore, as the LPT drives the propeller, a gearbox is required. Consequently, the lower the rotational speed, the heavier the reduction gearbox will be. 


\section{CHAPTER 3. DESIGN METHODOLOGY}

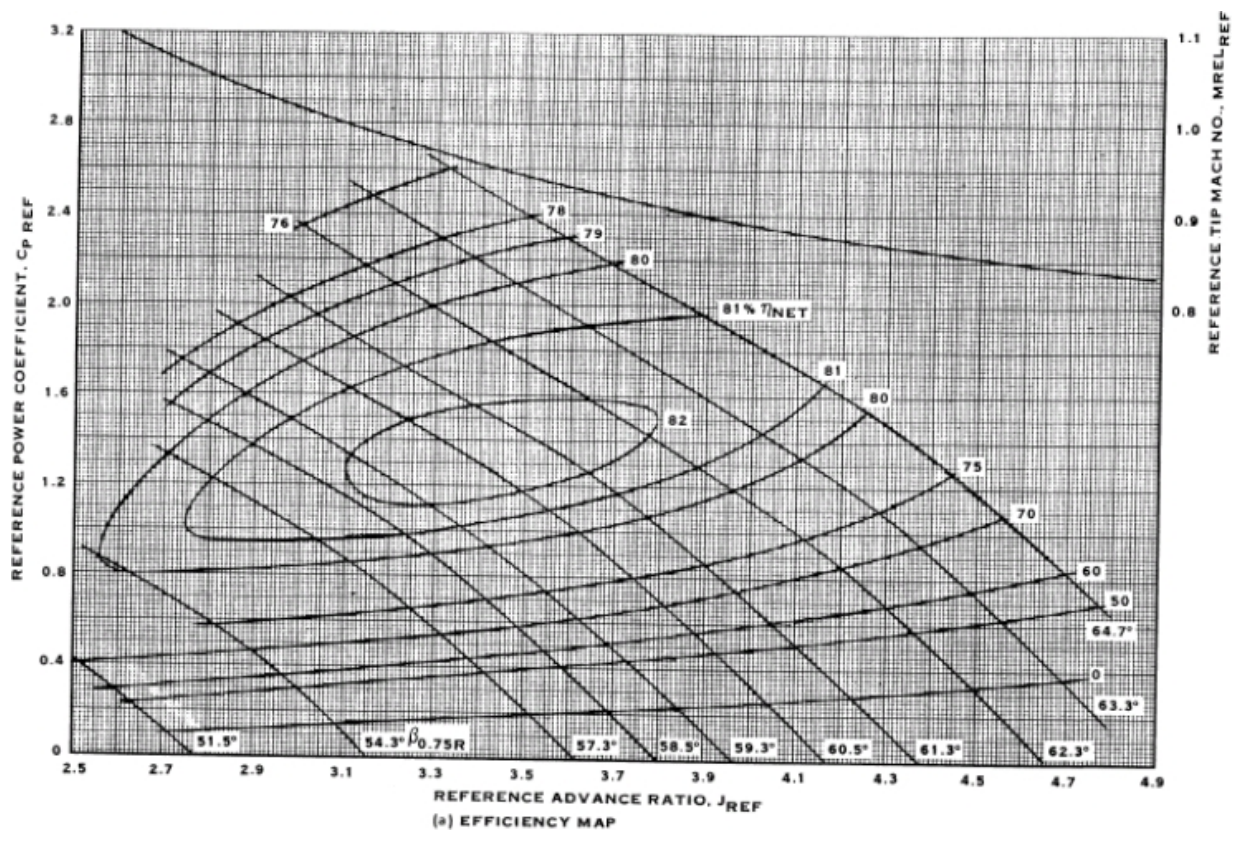

Figure 3.8: $45^{\circ}$ swept eight-bladed propeller map at $M_{\infty}=0.70$ (from Rohrbach et al. (1982)).

\subsubsection{GasTurb}

GasTurb ${ }^{1}$ is used for the analysis of the thermodynamics of gas turbines. It covers important aspects such as design cycle selection and off-design behaviour. Real compressor, turbine and propeller maps are used to yield representative results. For off-design calculations, iterative methods are implemented.

\section{Design Cycle}

The design cycle calculation determines the mass flows, total pressures and temperatures at the inlet and exit of all components, and the exhaust flow area. Furthermore, when the flow Mach number at the component boundaries is selected, the dimensions of the gas turbine are fixed. The design approach for turbofan and turboprop engines is discussed in the following.

First, a number of assumptions are applied to both engine types considered:

- kerosene fuelled, the fuel having a calorific value of $43.1 \mathrm{MJ} / \mathrm{kg}$;

- isentropic component efficiencies, for simplicity;

\footnotetext{
${ }^{1}$ www.gasturb.de
} 
- compressor and burner exit temperatures restricted (by current technology levels) to $900 \mathrm{~K}$ and $1800 \mathrm{~K}$, respectively (Cumpsty (1997));

- auxiliary power off-take of $50 \mathrm{~kW}$ per engine (default value in GasTurb for turbofan engines).

The temperature limits are most likely to be breached at takeoff; therefore, design-point values will be reduced to the maximum allowable, in order to yield the highest thermal efficiencies possible. Note, in GasTurb, auxiliary power off-takes, including the power to drive the suction pumps, are supplied by the high pressure turbine.

\section{Turbofan}

A two-spool engine is assumed, as opposed to a three-spool engine, for simplicity. Default values of 2.5 for the inner fan and 1.8 for the outer fan pressure ratios are also assumed. The approach to the turbofan design problem is as follows:

a) required power off-take is specified;

b) engine mass flow rate and high-pressure compressor (HPC) pressure ratio are iterated until the thrust requirement and limit on compressor exit temperature are reached, respectively;

c) the design bypass ratio (BPR) is set to avoid significant departures from the ideal jet velocity ratio;

d) off-design performance is evaluated through specification of the thrust and power offtake requirements;

e) compressor map is rescaled (discussed below), if necessary, to: 1) improve efficiency at cruise and 2), negate issues pertaining to close proximity of off-design points to the surge/stall line.

\section{Turboprop}

A two-spool turboprop engine is chosen over a single spool. The main difference between the two types is the transmission of power: with two spools, the propeller is driven by a dedicated turbine, the low pressure turbine. There are no obvious advantages of choosing three spools over two, unless the compressor pressure ratios are excessive. The design approach is similar to that for the turbofan, however differs in that there is a propeller. Therefore, step c), above, is replaced by 


\section{CHAPTER 3. DESIGN METHODOLOGY}

- blade diameter and rotational speed are chosen to ensure that the power loading and thrust coefficients are not excessive, whilst avoiding compressibility issues;

- propeller efficiency in cruise is maximised by rescaling the design point advance ratio and power coefficient in the propeller map (provided by GasTurb), assuming that the blade angle, pitch etc can be varied to obtain a realistic propeller efficiency.

\section{Compressor, Turbine and Propeller Maps}

Before proceeding with the off-design calculations, the design point must be correlated with the compressor and turbine maps. Standard maps are used in this study. These are supplied by GasTurb, and are taken from open literature. In the GasTurb user manual, it is explained that the maps may be scaled, e.g. to increase the surge margin, provided the compressor design pressure ratio does not deviate significantly from the original map. However, a consequence of this is that the efficiency contours are also rescaled; as the design cycle efficiency must remain the same, there is a tradeoff between high compressor surge margin and good efficiency off-design.

There are two scaling parameters: a non-dimensional speed $N / \sqrt{T}$ and an auxiliary coordinate $\beta$. These lie in the pressure-ratio - corrected-flow plane, and are shown in Fig. 3.9. $\beta$-lines are defined in GasTurb as "lines of pressure ratio versus mass flow which result in unique intersections with the speed lines", with values between $0-1$, as indicated in the figure.

The turbine scaling parameters are defined in an identical manner to those for the compressor. However, map rescaling is unlikely to be necessary, as the turbine blades operate under a favourable pressure gradient, and therefore do not posit a constraint.

Finally, GasTurb also provides a standard propeller map defined in the $C_{p}-J$ plane. To match this propeller map with that provided in Fig. 3.8, $C_{p}$ and $J$ are rescaled such that the maximum efficiency contours are at approximately the same values. 


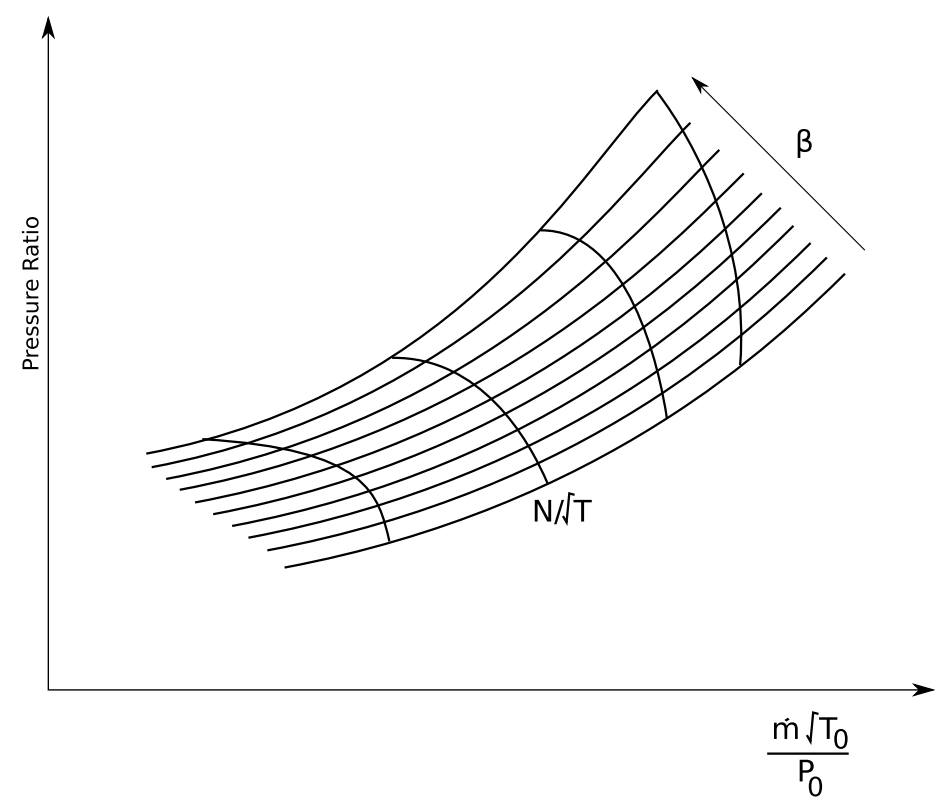

Figure 3.9: Compressor map scaling parameters (from GasTurb manual).

\section{Off-Design}

Once an engine has been sized based on the design cycle calculation, the analysis for each off-design point requires iteration. GasTurb does this by estimating several input iteration variables for the thermodynamic cycle. After each iteration, the imperfect estimates for the variables yield a set of inconsistencies in the thermodynamic analysis, which are referred to as iteration errors. With the total number of errors equal to the number of variables, GasTurb implements a Newton-Raphson solver to determine the change in the iteration variable required to drive the error to zero. A brief outline is provided here for the two engine types considered in this study.

\section{Turboprop}

The iteration variables and errors for a two-spool turboprop are summarised in Tab. 3.1. The first three iteration variables: $\beta_{C}, N_{C}$ and $\beta_{H P T}$, are combined with the inlet conditions, and compressor and turbine maps, to determine the compressor flow rate, burner inlet conditions, HPT flow rate and HPT work. The difference between the burner exit flow rate and HPT flow rate gives the first error; furthermore, as the HPT and compressor work (plus any power off-take) must be equal, any difference leads to the second error. With $N_{L P T}$ as an input variable (equal to a default value in GasTurb), the fourth iteration variable $\beta_{L P T}$ is combined 


\section{CHAPTER 3. DESIGN METHODOLOGY}

with the LPT map to determine the flow rate through the LPT. This is also derived from a second source, and the difference between two approaches gives the third error. With the nozzle inlet conditions now fixed, the total pressure in the core nozzle $P_{08}$ can be determined; however, for a choked nozzle of a certain area, the total pressure $P_{08 \text {,required }}$ to drive the flow gives a difference that sets up the fourth error. Finally, a fifth iteration variable is required, $T_{04}$, to achieve the required thrust specification.

Table 3.1: Summary of two-spool turboprop iteration variables and errors.

\begin{tabular}{cc}
\hline \hline Iteration variable & Error \\
\hline$\beta_{C}$ & HPT flow \\
$N_{C}$ & HPT work \\
$\beta_{H P T}$ & \\
\hline$\beta_{L P T}$ & LPT flow \\
& $P_{08}-P_{08, \text { required }}$ \\
\hline$T_{04}$ & Thrust requirement \\
\hline \hline
\end{tabular}

\section{Turbofan}

Table 3.2 summarises the iteration variables and errors for a two-spool, unmixed flow, turbofan engine. The compressor inlet conditions are combined with the LPC map, which is read through the first two iteration variables $\beta_{L P C}$ and $N_{L P C}$, to determine the LPC exit conditions. Furthermore, the total mass flow rate is derived from the map - the split between the bypass and core streams are defined by the third iteration variable $\dot{m}_{c} / \dot{m}_{b}$. With the rotational speed of the HPC, $N_{H P C}$, assumed to be an input (equal to a default value in GasTurb), the HPC map is read with the fourth iteration variable $\beta_{H P C}$. There is now enough information to determine the first error: the difference between the LPC and HPC flow rates. With the burner inlet conditions known, the burner exit temperature $T_{04}$ is the fifth iteration variable; this is combined with the sixth iteration variable $\beta_{H P T}$, which now sets the HPT map (as $N_{H P C}$ known), to determine the HPT work - the difference between the HPC (plus any power-off take) and HPT work is the third error. With the LPT connected to the LPC, the seventh iteration variable $\beta_{L P C}$ is now enough to read the LPC map, and determine the remaining errors, which include differences between: the LPT flow rate from the map and that from a second source; the LPC and LPT work; and $P_{08}$ and $P_{08 \text {,required }}$ (see above); the bypass stream provides the final error, i.e. the difference between $P_{18}$ and $P_{18 \text {,required }}$, which is the total pressure required to force the flow through the nozzle of area $A_{18}$. 
Table 3.2: Summary of two-spool, unmixed flow, turbofan iteration variables and errors.

\begin{tabular}{cc}
\hline \hline Iteration variable & Error \\
\hline$\beta_{L P C}$ & HPC flow \\
$N_{L P C}$ & \\
$\dot{m}_{c} / \dot{m}_{b}$ & \\
$\beta_{H P C}$ & \\
\hline$T_{04}$ & HPT flow \\
$\beta_{H P T}$ & HPT work \\
\hline$\beta_{L P T}$ & LPT flow \\
& LPT work \\
& $P_{08}-P_{08, \text { required }}$ \\
& $P_{18}-P_{18, \text { required }}$ \\
\hline \hline
\end{tabular}

\subsubsection{Structures and Weights}

The structural weight is evaluated by sizing primary structural elements on the basis of their loading, using standard analytic methods detailed by Howe (2004) and Greitzer et al. (2010). In this section, an overview of the methods used for sizing conventional aircraft are discussed, and then adapted for an analysis of the LFW aircraft. Finally, the approach is applied to a conventional aircraft with a known weight breakdown for validation.

\subsubsection{Methodology}

The proposed structural design methodology is summarised in Fig. 3.10. First, external loads are identified. The aerodynamic loadings resulting from a manoeuvre and a gust are then assessed, and the critical case identified. The primary and secondary structural elements of the fuselage, wing and tailplane are sized, assuming a conventional Aluminium Alloy 2024T3 and a safety factor $N_{s}$ of 1.5. (There are potential benefits in using composite materials, however to exploit these would require going beyond the initial sizing calculations presented here.) A conservative first estimate of the structural weight follows. The structural elements are resized to allow for inertial relief with the inclusion of self-weight, and the process is iterated until convergence of the final weight estimate. Finally, the structural forces on landing are assessed, and additional structural material is added if necessary.

\subsubsection{Loading Conditions}

A summary of the design airworthiness requirements is provided by Jenkinson et al. (1999). The key elements considered in this study are: 


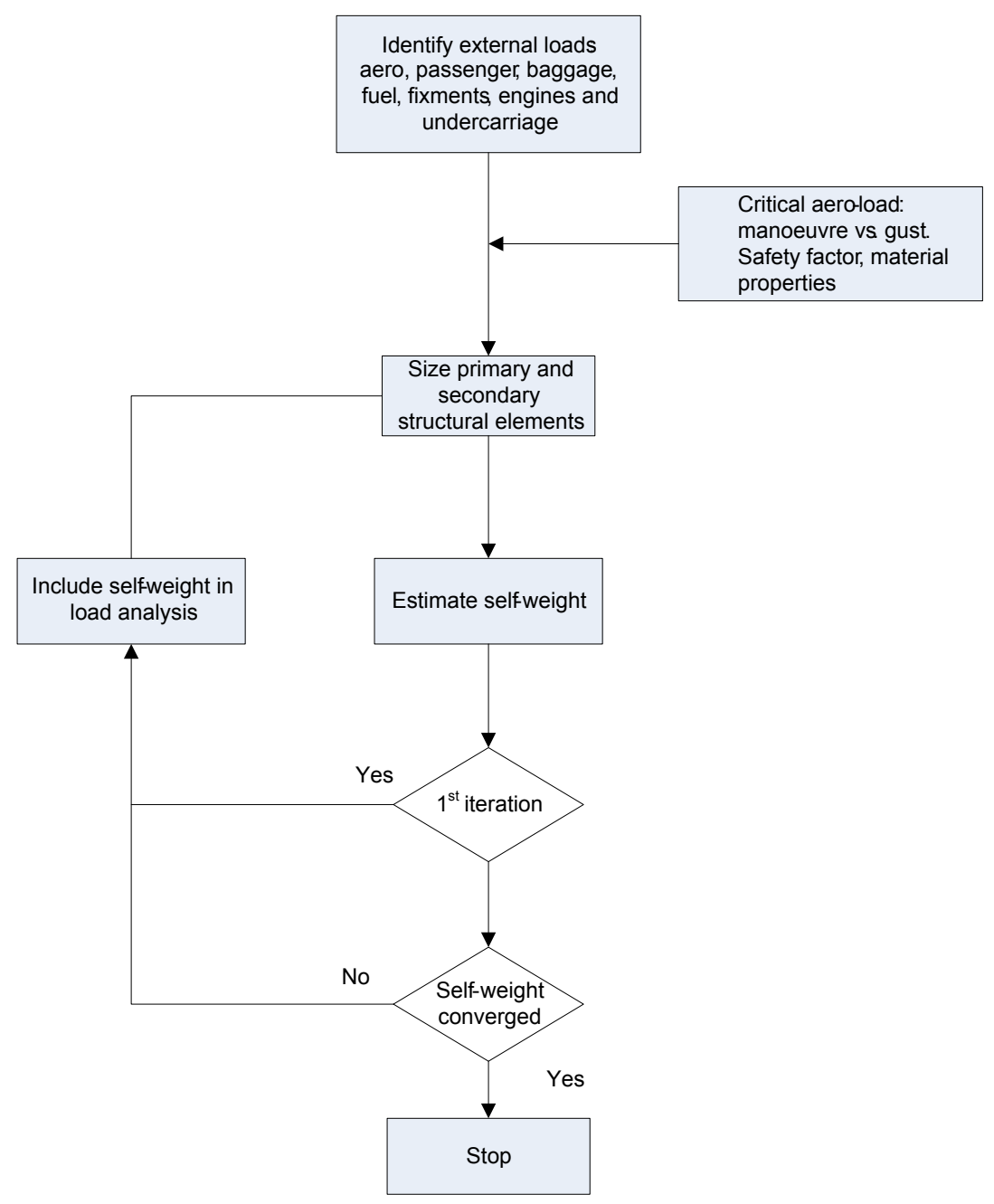

Figure 3.10: Schematic of structural design methodology.

1. Flight manoeuvre, gust and ground loads;

2. Supplementary conditions (e.g. pressurisation, unsymmetrical loads);

3. Control surface and system loads.

A load factor of 3 -g for landing conditions is stipulated in FAR regulations ${ }^{1}$. Meanwhile, the acceleration arising from a sharp manoeuvre is generally considered the most critical aero-load scenario for conventional aircraft - Raymer (1999) gives a value of around 2.5-g. However, gust loads, assumed uniform across the span and acting parallel to the vertical

\footnotetext{
${ }^{1}$ http://www.flightsimaviation.com/data/FARS/part_23-561.html
} 
axis of the aircraft can be higher, especially for lightly loaded aircraft. The load factor, $\Delta n$, experienced due to a vertical gust is given by

$$
\Delta n=\frac{\Delta L}{W}=\frac{\rho U_{g} U_{\infty} C_{L \alpha}}{2 W / S},
$$

where the gust velocity $U_{g}$ is determined from statistical data.

System loads include items such as: engines, suction hardware, fixed equipment, avionics, auxiliary power units, and nose and main landing gears. A passenger weight of $80 \mathrm{~kg}$ plus 20 $\mathrm{kg}$ of luggage is specified, on the basis of the study of Hileman et al. (2007). The landinggear weight is estimated, following Hileman et al. (2007), at 4\% MTOW, with $10 \%$ of this allocated to the nose gear (Raymer (1999)). Fixed-equipment weight consists of items such as avionics, APU, etc, and is assumed equal to the payload weight (see Hileman et al. (2007)); of this, $10 \%$ is allocated to the avionics and $3.5 \%$ to the APU.

The internal pressurisation of the fuselage is governed by FAR codes and is set at 0.75 bar for flight altitudes above $8000 \mathrm{ft}$ (FAR Sec. 25.841). This in turn determines the pressure difference across the cabin skin at the design altitude.

\subsubsection{Fuselage Structure}

In Chap. 2 we saw that the fuselage structure of a flying wing is quite different to a conventional aircraft. The former utilises a multi-bubble shell configuration, whilst the latter has a cylindrical pressure vessel. Both are subject to pressurisation and distributed loads; however, additional horizontal and vertical loads must be considered for tube-and-wing aircraft.

\section{Multi-Bubble Pressure Vessel}

\section{Geometry}

Figure 3.11 shows a cross section of the multi-bubble pressure vessel perpendicular to the spanwise axis. For N cylindrical bubbles, there are N-1 vertical bulkheads of height $2 h_{f, v b}$ and thickness $t_{f, v b}$. Each cylinder has a radius $R_{f}$ and skin thickness $t_{f, s}$, and the overall section area is $A_{f, s}$. The bubbles either terminate at wing spar locations, or are cylindrical.

The shell has the following geometric and section-area relations:

$$
\begin{aligned}
\theta_{f, v b} & =\arcsin \left(\sqrt{1-\left(\frac{h_{f, v b}}{R_{f}}\right)^{2}}\right) \\
A_{f, s} & =2\left(\theta_{f, 1}+\theta_{f, 2}\right) R_{f} t_{f, s}+(N-1) 4 \theta_{f, v b} R_{f} t_{f, s} \\
A_{f, v b} & =2(N-1) h_{f, v b} t_{f, v b}+2 R_{f} t_{f, v b}\left(\cos \theta_{f, 1}+\cos \theta_{f, 2}\right)
\end{aligned}
$$


The total fuselage cross-sectional area and surface area of the cabin shell are denoted by $A_{f}$ and $S_{f, m b}$, respectively:

$$
\begin{aligned}
A_{f}= & \left(\theta_{f, 1}+\theta_{f, 2}+\frac{1}{2}\left(\sin 2 \theta_{f, 1}+\sin 2 \theta_{f, 2}\right)\right) R_{f}^{2} \\
& +(N-1)\left(2 \theta_{f, v b}+\sin 2 \theta_{f, v b}\right) R_{f}^{2} \\
S_{f, m b}= & 2\left(\theta_{f, 1}+\theta_{f, 2}\right) R_{f} l_{f, m b}+2(N-1) l_{f, m b} R_{f} \theta_{f, v b}
\end{aligned}
$$

where $l_{f, m b}$ is the spanwise length of the cabin (as shown in Fig. 3.12).

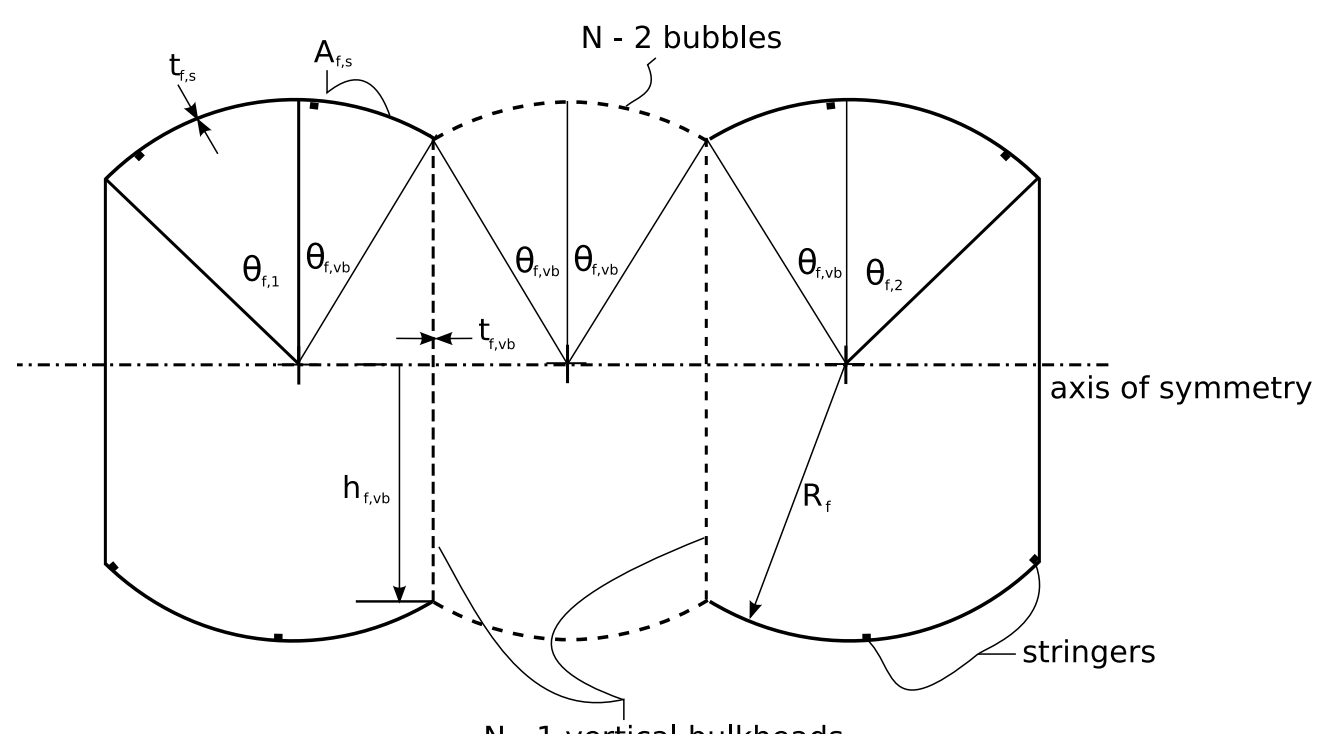

$\mathrm{N}$ - 1 vertical bulkheads

Figure 3.11: Multi-bubble geometric parameters (perpendicular to the spanwise axis).

Figure 3.12 shows a cross section of the fuselage perpendicular to the chordwise axis. The multi-bubble cabin section is capped off from the unpressurised regions at the spanwise extremes by domed bulkheads of radius $R_{f}$ and thickness $t_{f, d b}$. Wing ribs (fuselage frames) extend through the lower surface to support the floor.

The dome-bulkhead surface area $S_{f, d b}$ is an extension of the approximation for a spherical bulkhead given by Greitzer et al. (2010):

$$
S_{f, d b} \simeq 2\left(\left(\theta_{f, 1}+\theta_{f, 2}\right) R_{f}^{2}+(N-1) 4 \theta_{f, v b} R_{f}^{2}\right)
$$




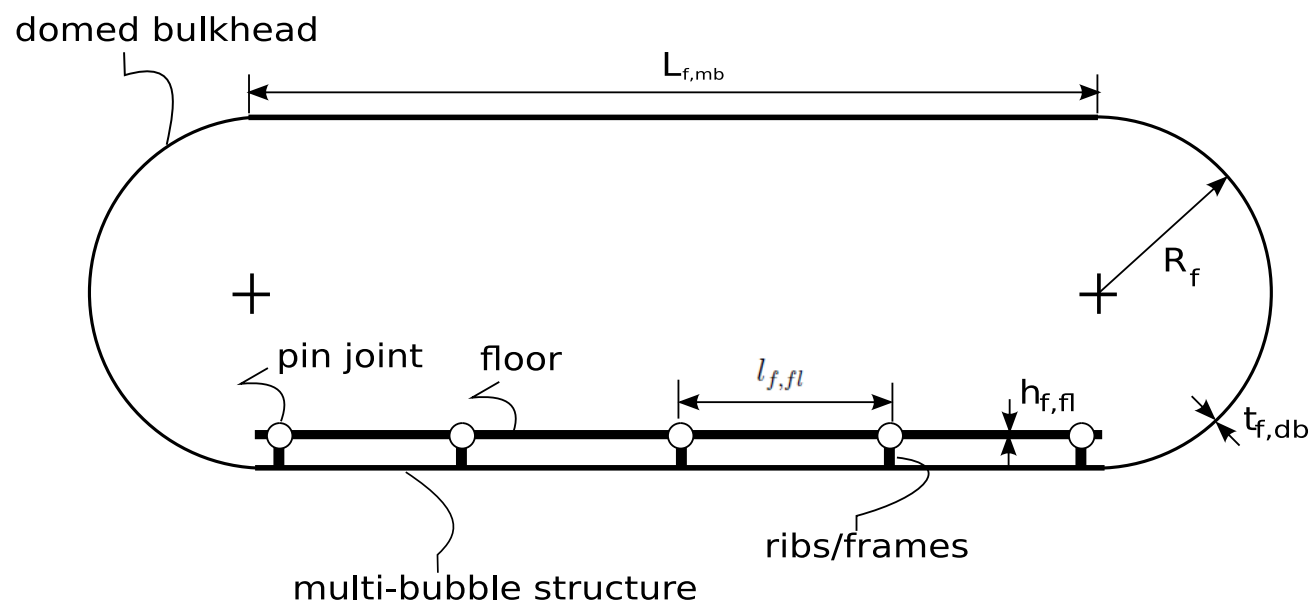

Figure 3.12: Cabin cross section (perpendicular to the chordwise axis).

Shell

The skin resists internal pressurisation in hoop-wise tension provided vertical bulkheads are placed at the 'bubble' joins. The axial and hoop-wise skin stresses are:

$$
\begin{aligned}
\sigma_{x x} & =N_{s} \Delta P \frac{A_{f}}{A_{f, s}+A_{f, v b}} \\
\sigma_{\theta \theta} & =N_{s} \Delta P \frac{R_{f}}{t_{f, s}}
\end{aligned}
$$

The ratio of these two stresses, as a function of the various geometric parameters that define the multi-bubble pressure vessel, is:

$$
\frac{\sigma_{x x}}{\sigma_{\theta \theta}}=\frac{1}{2}\left(\frac{\theta_{f, 1}+\theta_{f, 2}+(N-1)\left(2 \theta_{f, v b}+\sin 2 \theta_{f, v b}\right)}{\theta_{f, 1}+\theta_{f, 2}+(N-1)\left(2 \theta_{f, v b}+\cos \theta_{f, v b}\right)}\right) .
$$

The ratio $\frac{\sigma_{x x}}{\sigma_{\theta \theta}}$ is plotted against $\theta_{f, v b}$ for extreme values of $N$ in Fig. 3.13 for the case where $\theta_{f, 1}=\theta_{f, 2}=\pi / 2$. The figure shows that for the cylindrical case, i.e. one bubble, $\sigma_{x x} / \sigma_{\theta \theta}=$ $1 / 2$, as expected; in contrast, for a large number of bubbles $(N=20)$, the maximum value of $\sigma_{x x} / \sigma_{\theta \theta}$ does not exceed around 0.6. Consequently, the skin thickness must be sized by the larger, hoop-wise, stress to meet the allowable stress $\sigma_{f, s}$ :

$$
t_{f, s}=N_{s} \Delta P \frac{R_{f}}{\sigma_{f, s}} .
$$

The hoop-wise membrane forces T make an angle $\phi$ with the vertical, and are reacted by a tensile force $\mathrm{F}$ in the vertical bulkhead at the multi-bubble joins, as illustrated in Fig. 3.14. 


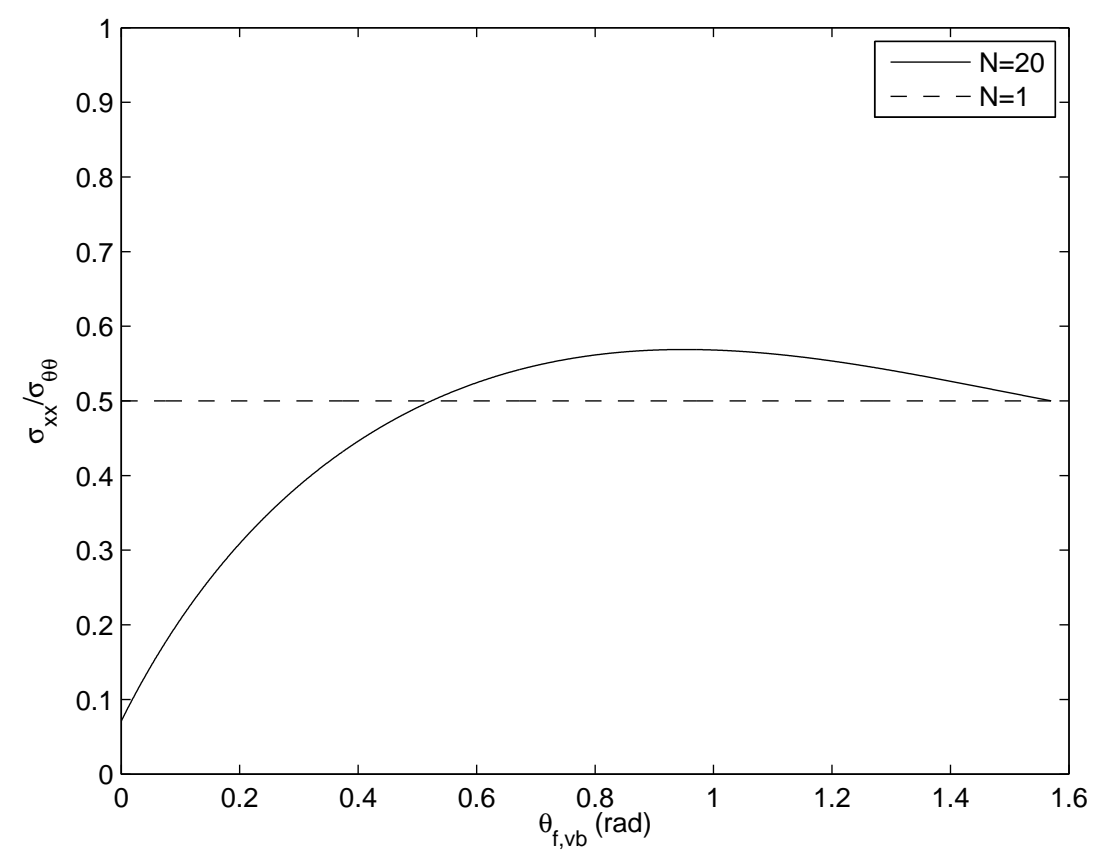

Figure 3.13: Variation of the ratio of axial-to-hoopwise stress with $\theta_{f, v b}$ and $N$ for $\theta_{f, 1}=$ $\theta_{f, 2}=\pi / 2$.

Assuming both components have the same material properties, the vertical bulkhead thickness

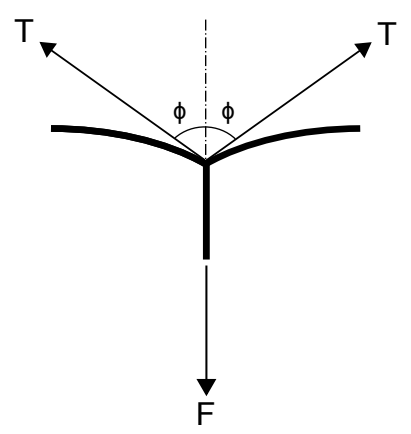

Figure 3.14: Vertical bulkhead force.

is given by

$$
t_{f, v b}=2 t_{f, s} \cos \phi
$$

where $\phi=\pi / 2-\theta_{f, v b}$.

For a cylindrical pressure vessel with a semi-hemispherical end-cap, the stress in the cap is half the hoop-wise stress, and therefore the cap thickness is half the cylinder thickness. Such 
a discontinuity induces local bending moments, and a transition is required. Therefore, it is conservatively assumed that the domed-bulkhead thickness $\left(t_{f, d b}\right)$ is equal to the fuselage-skin thickness $\left(t_{f, s}\right)$.

The volumes of material required for the domed bulkheads, multi-bubble cylinders and vertical bulkheads are:

$$
\begin{aligned}
\nu_{f, d b} & =S_{f, d b} t_{f, s} \\
\mathcal{V}_{f, m b} & =A_{f, s} l_{f, m b} \\
\mathcal{V}_{f, v b} & =A_{f, v b} l_{f, m b}
\end{aligned}
$$

A breakdown of the shell weight is therefore:

$$
\begin{aligned}
W_{f, s} & =\rho_{a l} g\left(\mathcal{V}_{f, m b}+\mathcal{V}_{f, d b}\right) \\
W_{f, v b} & =\rho_{a l} g \mathcal{V}_{f, v b} \\
W_{f, s h} & =W_{f, s}\left(1+f_{f, s t}\right)+W_{f, v b}
\end{aligned}
$$

where $\rho_{a l}$ is the material density, and the weight fraction of the stringers is given by Howe (2004) to be $f_{f, s t}=0.25$.

Floor

Transverse floor beams are required to support the payload and additional weights (seats, galleys, etc), which are assumed uniformly distributed, and have a weight per unit area

$$
\mathcal{W}_{f, f l}=N_{l} N_{s} \frac{\left(W_{p a y}+W_{f i x}\right)}{\left(l_{f, m b}+2 R_{f}\right) w_{f, f l}}
$$

In sizing the floor beams, the emergency landing case $\left(N_{l}=3\right)$ is taken as critical.

Figure 3.12 shows that the floor joints are modelled as pinned, with a separation $l_{f, f l}$ equal to the rib/frame pitch. This arrangement leads to a more conservative weight estimate than a clamped model would. The load due to the floor self-weight is neglected, as it is typically much smaller than the payload. The maximum shear force and bending moment expressions are subsequently

$$
\begin{aligned}
\mathcal{S}_{f, f l} & =\frac{1}{2} w_{f, f l} l_{f, f l} \mathcal{W}_{f, f l} \\
\mathcal{M}_{f, f l} & =\frac{1}{8} w_{f, f l} l_{f, f l}^{2} \mathcal{W}_{f, f l}
\end{aligned}
$$




\section{CHAPTER 3. DESIGN METHODOLOGY}

where the floor width

$$
w_{f, f l}=2(N-1) R_{f} \sin \theta_{f, v b}+R_{f} \sin \theta_{f, 1}+R_{f} \sin \theta_{f, 2}
$$

For a set of floor I-beams with height $h_{f, f l}$, the total average cross-sectional area and corresponding volume of beam material are given by Greitzer et al. (2010) as:

$$
\begin{aligned}
A_{f, f l} & =\frac{2.0 \mathcal{M}_{f, f l}}{\sigma_{f, f l} h_{f, f l}}+\frac{1.5 \mathcal{S}_{f, f l}}{\tau_{f, f l}} \\
\nu_{f, f l} & =w_{f, f l} A_{f, f l}
\end{aligned}
$$

(The coefficients of 2.0 and 1.5 assume no tapering of the cross section; the floor height $h_{f, f l}$ is assumed to be $10 \mathrm{~cm}$.) The floor weight is then

$$
W_{f, f l}=\rho_{a l} g \mathcal{V}_{f, f l}+w_{f, f l}\left(l_{f, m b}+2 R_{f}\right) \mathcal{W}_{f, f l-p l}
$$

where $\mathcal{W}_{f, f l-p l}$ is a specified floor-plank weight density.

\section{Cylindrical Pressure Vessel}

The reader is referred to the study of Greitzer et al. (2010), as this is followed directly. However, a short summary of the sizing method follows.

The model consists of a cylindrical pressure vessel with an ellipsoidal nose endcap and a hemispherical tail endcap. The fuselage is subjected to internal pressurisation $\Delta P$, vertical $\left(\mathcal{M}_{v}\right)$ and horizontal $\left(\mathcal{M}_{h}\right)$ bending, and torsion $\left(Q_{v}\right)$ loads. These are depicted in Fig. 3.15. (In this study, the added weight $W_{\text {padd }}$ and seat weight $W_{\text {seat }}$ are not treated in the same way. Instead, a fixed equipment weight is included $W_{f i x}$.)

The skin thickness is sized based on the hoop-wise skin stress that results from cabin internal pressurisation. Reinforcements such as frames and stringers are assumed to contribute $25 \%$ each to the total shell weight (Howe (2004)). Additional vertical and horizontal bending material is added wherever the resulting bending moments exceed the capability of the pressure vessel's thickness based on pressurisation considerations alone. The schematic diagram in Fig. 3.15 (a) shows that the moments acting over the front and rear of the fuselage match at the location $x_{\text {wing }}$, which for simplicity is taken as the wing's area centroid. The distributed loading, weight of the tailplane $N W_{\text {tail }}$ and aerodynamic loading acting on the horizontal tailplane $r_{M h} L_{h}$ contribute to $\mathcal{M}_{h}$. Two scenarios are considered: in-flight at the maximum load factor, and an emergency landing impact. Meanwhile, $\mathcal{M}_{v}$ depends solely on the vertical fin loading $r_{M v} L_{v}$. (The factors $r_{M v}$ and $r_{M h}$ account for inertial relief.) 


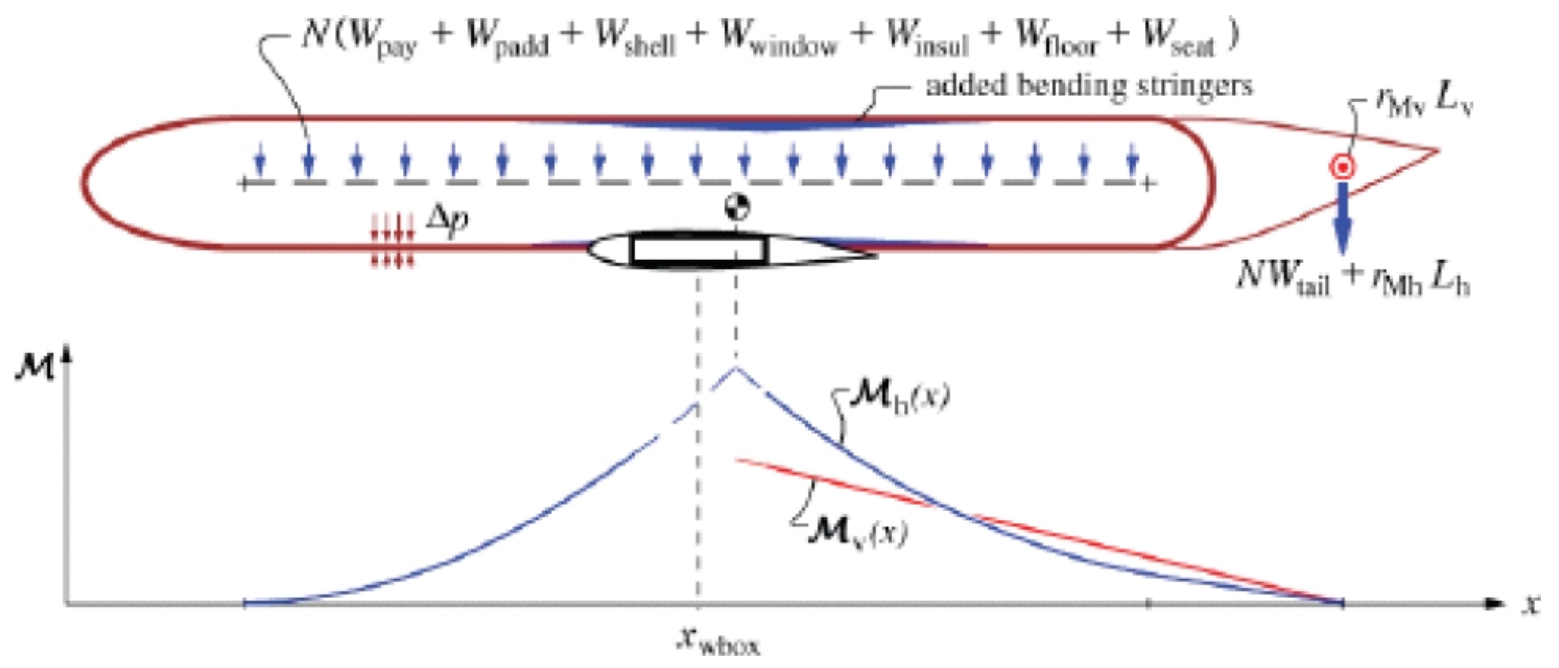

(a) Conventional fuselage layout, loads, and bending moment and inertia distributions.

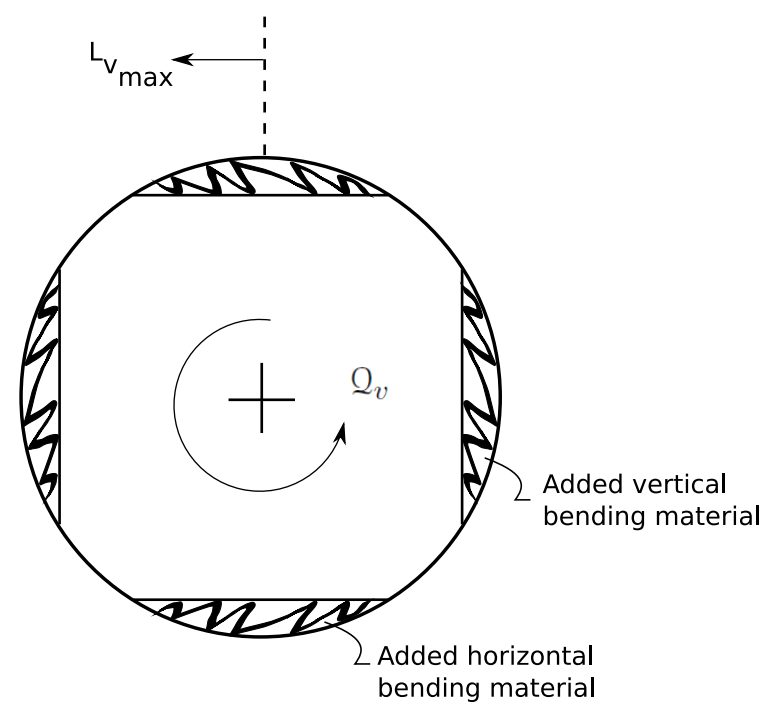

(b) Fuselage cross-section, and torsion load from vertical fin.

Figure 3.15: Conventional fuselage geometry and applied loads (from Greitzer et al. (2010)).

\section{Secondary Structures}

Secondary fuselage weights are given by empirical weight fractions:

1. Windows (conventional only): an area-distributed weight of $0.66 \mathrm{~kg} / \mathrm{m}^{2}$, estimated from the study of Greitzer et al. (2010).

2. Insulation: an area-distributed weight of $1.08 \mathrm{~kg} / \mathrm{m}^{2}$, estimated from the study of Greitzer et al. (2010). 


\section{CHAPTER 3. DESIGN METHODOLOGY}

3. An area-distributed floor-plank weight of $5 \mathrm{~kg} / \mathrm{m}^{2}$ (Torenbeek (1976)).

\subsubsection{Wing Structure}

The wing structure of the flying wing is sized following conventional methods given by Howe (2004). Instances where the analysis differs for the conventional configuration are highlighted; otherwise, the same methods are used to size that aircraft's wing, horizontal tailplane and vertical fin.

\section{Overview of Structural Elements}

Figure 3.16 shows the primary structural elements of the wing. The skin provides the required aerodynamic shape whilst, structurally, the upper and lower surfaces act as the main carriers of longitudinal bending; a proportion is also reacted by spanwise stringers. (Stringers also serve to reduce the size of areas of unsupported skin, thereby reducing local buckling loads and aiding restriction of potential deformations.) Spar webs are positioned at the extremities of the wing-box section. In a two-spar construction, Howe (2004) advises that the front spar should be as far forward as possible subject to there being enough wing depth to efficiently carry vertical shear loads, whilst the rear spar should be as far aft as possible such that control surfaces can be accommodated and also have sufficient depth. Chordwise ribs help maintain the aerodynamic shape of the wing cross-section, transmit local loads chordwise across to the spanwise beams (spars), and limit the length of the skin-stringer panel arrangement to an efficient column compressive strength. Additional secondary components such as slats, flaps, spoilers and control surfaces make up the remaining structural weight.

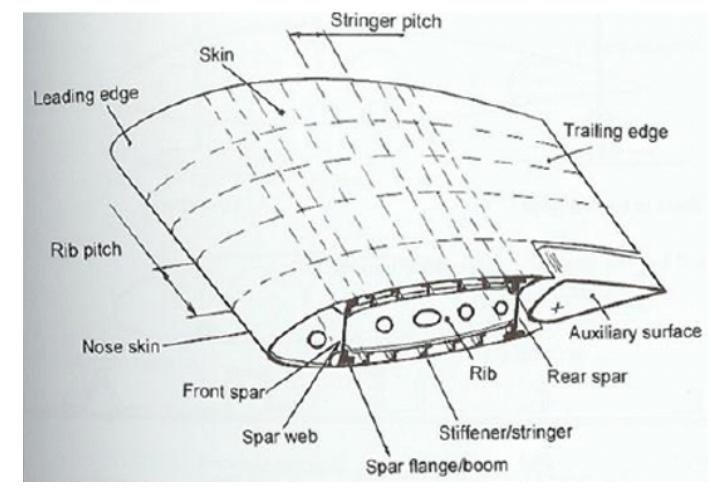

Figure 3.16: Wing construction (from Howe (2004)). 


\section{Geometry}

A schematic diagram of a typical wing and wingtip fin configuration is provided in Fig. 3.17. To complement a discussion of the wing structural forces and stresses, four spanwise stations and the reference axes, which coincide with the wing-box shear-centre locus, are detailed. The reference axes are inclined at an angle $\Lambda$ relative to the axis normal to the centreline, and are of length $L_{w}$. The resolved chord length $c_{\perp}$ is detailed, and lies perpendicular to the reference axis. A structurally efficient layout would consist of a single spar-web construction running along the span (i.e. $\Lambda_{1-2}=\Lambda_{2-3}$ ); however, if geometric constraints do not permit this, a kinked spar-web construction would follow.

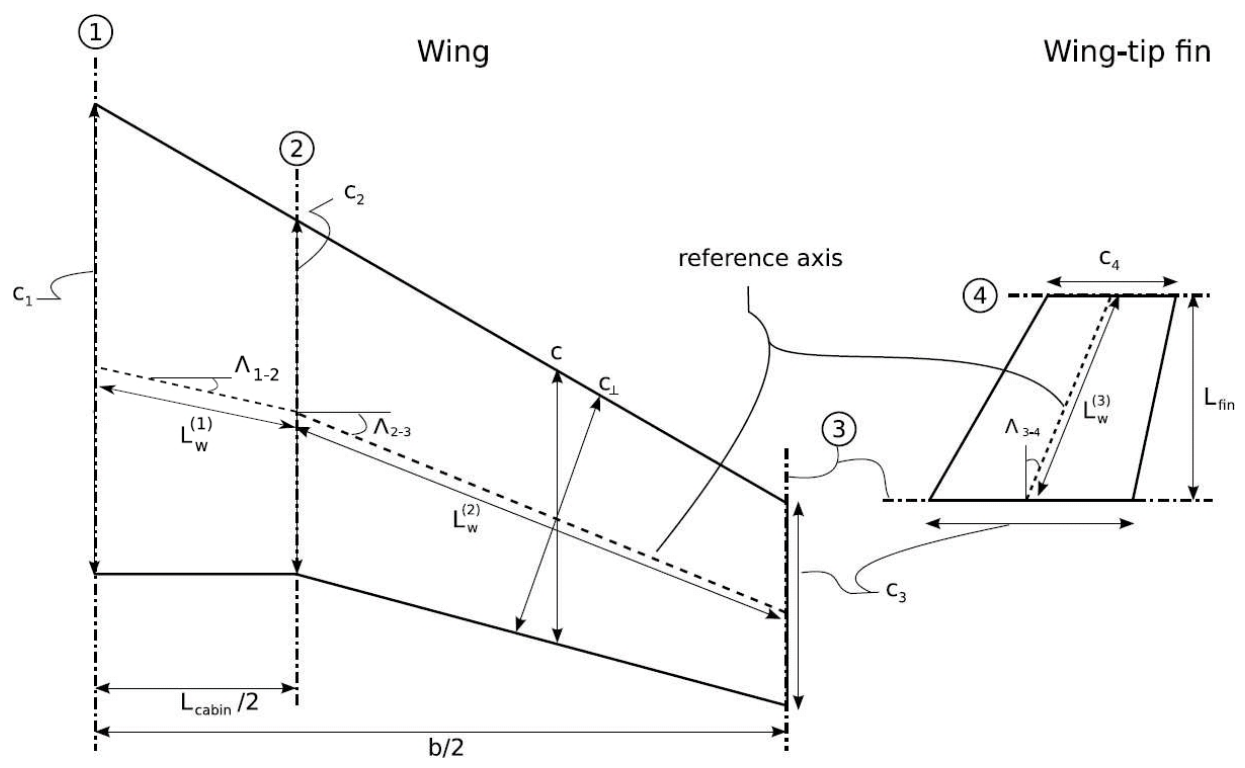

Figure 3.17: Wing (or tail) planform.

A cross section of the wing-box construction in the plane perpendicular to the reference axis is shown in Fig. 3.18. The wing-box depth $h_{w, b o x}$ and width $w_{w, b o x}$ at each spanwise station are used to evaluate the stresses, and hence the required thicknesses of flange and spar-web material $t_{w, f}$ and $t_{w, w}$, respectively. (Note, the flange thickness combines both the skin and effective stringer thickness.) The shear centre is located half-way between the front and rear spars, which are a distance $x_{\text {lesp }}$ and $x_{\text {tesp }}$, respectively, from the leading edge, as illustrated. (The leading and trailing edge regions are not detailed as they do not contribute to the wing-box structure analysis.) 


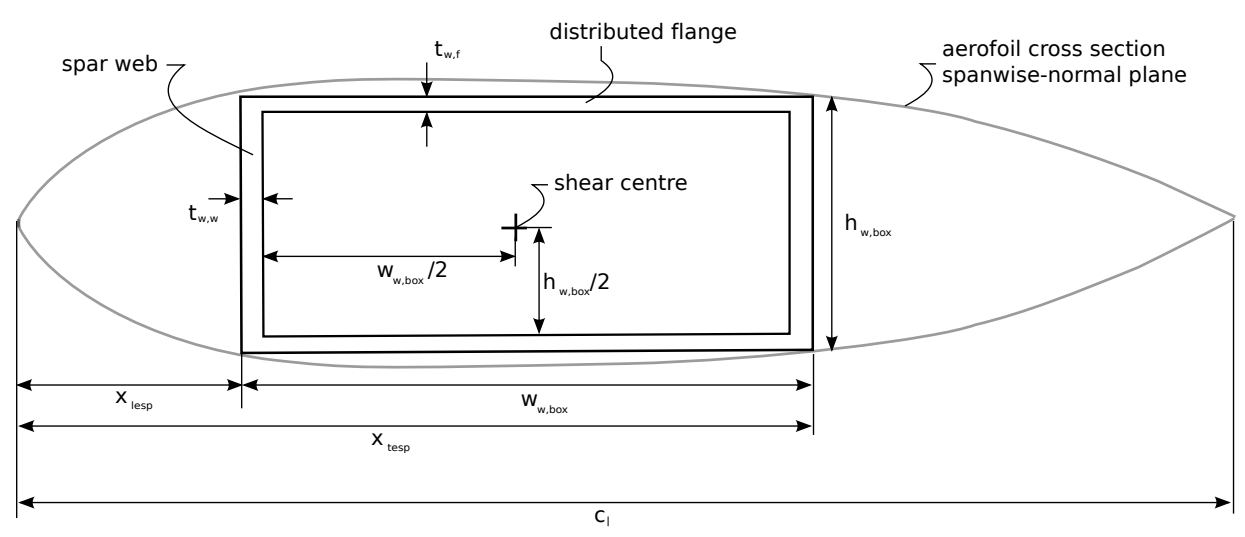

Figure 3.18: Wing (or tail) aerofoil and wing-box cross section (perpendicular to the reference axis).

\section{Stress and Weight Formulation}

In the following, an account of the analytical methods used to size the primary elements of the wing structure is given. Note, an index superscipt (' $j$ ') is used to show explicitly that forces, stresses and material thicknesses are evaluated at each spanwise station.

\section{Skin and Stringer Panels}

The wing skin is modelled as a distributed flange reinforced by Zed-stringer sections (Howe (2004)). Its size depends on the bending moment $\mathcal{M}_{w, s}^{(j)}$, or rather the equivalent axial force $\mathcal{P}_{w, s}^{(j)}$ in the upper and lower surfaces

$$
\mathcal{P}_{w, s}^{(j)}=\frac{\mathcal{P}_{w, s}^{(j)}}{h_{w, b o x}^{(j)}}
$$

Howe (2004) explains that for preliminary design studies, it is sufficient to assume that the skin reacts $65 \%$ of the bending load. Therefore, the wing-skin thickness is

$$
t_{w, s}^{(j)}=0.65 \frac{\mathcal{P}_{w, s}^{(j)}}{w_{w, b o x}^{(j)} \sigma_{w, s}^{(j)}},
$$

where $\sigma_{w, s}^{(j)}$ is the maximum allowable stress.

The allowable working stress $\sigma_{w, s}^{(j)}$ is dictated by buckling stability for the compression surface and fatigue/crack avoidance for the tensile surface: 
a) The minimum stress under which buckling occurs $\sigma_{w, b}^{(j)}$ in the compression surface for a given applied load $\mathcal{P}_{w, s}^{(j)}$ is

$$
\sigma_{w, b}^{(j)}=\bar{A} F_{b}\left(\frac{\mathcal{P}_{w, s}^{(j)}}{w_{w, b o x}^{(j)} p_{w, r}^{(j)}}\right)^{\frac{1}{2}}
$$

where: for aluminium $\bar{A}$ is 138 , and $F_{b}$ is 0.96 for a Zed stringer configuration; the loading intensity $\frac{\mathcal{P}_{w, s}^{(j)}}{w_{w, b o x}^{(j)} p_{w, r}^{(j)}}$ (unit $\left.\mathrm{MN} / \mathrm{m}^{2}\right)$ is the main parameter determining the allowable compression stress; $p_{w, r}^{(j)}$ is the support spacing along the axis of the beam, i.e. the rib/frame pitch. (Interestingly, by limiting the width of the wing box, a higher structural efficiency may be attained as higher working stresses can carried by the distributed flanges, though this would require sacrificing potential cabin, cargo and fuel volume.)

b) Ashby et al. (2001) give the number of cycles that the wing material must endure as $10^{7}$ (under aviation regulations), resulting in a (fatigue) stress limit $\sigma_{w, f}=0.30 \sigma_{u t s}$ for the tension surface.

Accounting for geometric tapering of the wing, the wing skin volume is

$$
\mathcal{V}_{w, s}^{(j)}=\left(t_{w, s}^{(j), c o m p}+t_{w, s}^{(j), t e n s}\right) \bar{w}_{w, b o x}^{(j)} L_{w}^{(j)} .
$$

where $\bar{w}_{w, b o x}^{(j)}=\left(w_{w, b o x}^{(j)}+w_{w, b o x}^{(j+1)}\right) / 2$. Hence the skin weight is given by

$$
W_{w, s}=\rho_{a l} g \sum_{j} V_{w, s}^{(j)}
$$

Stringers react the remaining $35 \%$ of the bending load, giving a 'smeared' area ratio for the stringers-to-skin of

$$
\frac{A_{w, s t}}{A_{w, s}}=0.54 .
$$

The weight of the stringer construction is

$$
W_{w, s t}=\rho_{a l} g \sum_{j} A_{w, s t}^{(j)} L_{w}^{(j)} .
$$




\section{CHAPTER 3. DESIGN METHODOLOGY}

\section{Spar Webs}

The spar-web thickness is sized to react vertical and torsional shear loads. The shear flow due to torsion around the wing-box section is

$$
Q_{w, w}^{\mathcal{T}_{w}^{(j)}}=\frac{\mathcal{T}_{w}^{(j)}}{2 A_{e}^{(j)}}
$$

where $A_{e}^{(j)}$ is the area enclosed by the wing-box section. For equal spar-web heights, the vertical shear in each web is half the total vertical shear acting on the wing section $\mathcal{S}_{w}^{(j)}$. The vertical shear flow is therefore

$$
Q_{w, w}^{\mathcal{S}^{(j)}}=\frac{\mathcal{S}_{w}^{(j)}}{2 h_{w, b o x}^{(j)}} .
$$

The direction of the shear flows is important. For example, if the torsion couple acts in a nose-up direction, the shear flows in the front spar will act to effectively cancel, whereas in the rear spar they reinforce one another. For a worst case estimate, both spars are sized according to the more critical condition.

The spar-web thickness is given by

$$
t_{w, w}^{(j)}=\frac{Q_{w, w}^{\mathcal{T}_{w}^{(j)}}+Q_{w, w}^{\mathcal{S}^{(j)}}}{\sigma_{w, w}^{(j)}} .
$$

(Assuming that the cover skins and internal webs do not buckle in shear, it is sufficient to assume that at ultimate conditions the shear stress in the web $\sigma_{w, w}^{(j)}=0.5 \sigma_{u t s}$.)

The spar-web volume, allowing for geometric tapering of the wing, is

$$
\mathcal{V}_{w, w}^{(j)}=2 t_{w, w}^{(j)} \bar{h}_{w, b o x}^{(j)} L_{w}^{(j)}
$$

where $\bar{h}_{w, w}^{(j)}=\left(h_{w, b o x}^{(j)}+h_{w, b o x}^{(j+1)}\right) / 2$. Therefore the weight of the spar-web construction is

$$
W_{w, w}=\rho_{a l} g \sum_{j} \mathcal{V}_{w, w}^{(j)}
$$

\section{Ribs}

The rib construction depends on its function. Across all regions the rib consists of a web (i.e. a thin metal sheet), serving to prevent buckling of the wing skin and withstanding dynamic forces arising from fuel slosh. In regions where the ribs must support: control surfaces at hinge attachment points, engines, and the landing gear, spars are required to react vertical 
shear loads, along with flanges to react torsional shear loads.

The rib flange and spar construction, with dimensions $w_{w, r_{f}}$ and $h_{w, r_{s p}}$, respectively, are illustrated in Fig. 3.19. It also shows the location of a point load $W_{p}$ relative to the shear centre.

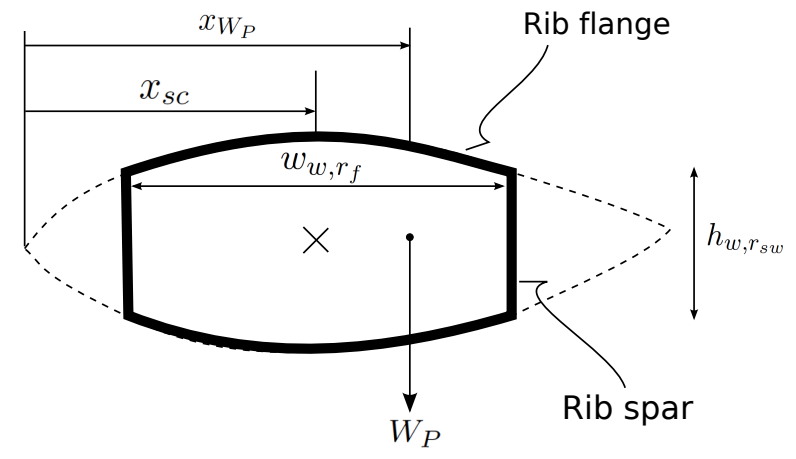

Figure 3.19: Schematic diagram of rib flange and spar construction.

For initial calculations, Howe (2004) advises that a satisfactory estimate of the rib pitch to prevent buckling of the wing skin may be estimated using the empirical correlation

$$
p_{w, r}^{(j)}=0.55\left(h_{w, r_{s w}}^{(j)}\right)^{1 / 2}
$$

Along the wing span, the section depth $h_{w, r_{s w}}$ varies due to tapering of the wing. Therefore, the required rib pitch to avoid buckling instability will subsequently change over each spanwise section. The number of ribs required is therefore

$$
N_{w, r}^{(j)}=\frac{L_{w}^{(j)}}{p_{w, r}^{(j)}} .
$$

The rib webs are assumed to have a thickness equal to the minimum used in industry to withstand fuel slosh loads; Niu (1988) gives $t_{w, r_{w}}=1 \mathrm{~mm}$. The ribs are aligned orthogonally to the reference axis, and therefore have a length equal to the mean perpendicular chord length over a spanwise region. The weight of the rib webs is consequently

$$
W_{w, r_{w}}=\rho_{a l} g \sum_{j} N_{w, r}^{(j)} t_{w, r_{w}} \bar{h}_{w, r_{s w}}^{(j)} \bar{c}_{\perp}^{(j)}
$$

where $\bar{c}_{\perp}^{(j)}=\left(c_{\perp}^{(j)}+c_{\perp}^{(j+1)}\right) / 2$.

The rib spars are sized following the same method detailed for the wing spar webs. The 
rib spar weight is

$$
W_{w, r_{s w}}=2 \rho_{a l} g\left(N_{w, r_{e}} t_{w, r_{e}}^{2} \bar{h}_{w, r_{s p}}+N_{w, r_{u c}} t_{w, r_{u c}}^{2} \bar{h}_{w, r_{s p}}\right)
$$

where $t_{w, r_{e}}$ and $t_{w, r_{u c}}$ denote the spar material thickness corresponding to the engine and undercarriage, respectively, and are assumed to have square cross sections; $N_{w, r_{e}}$ and $N_{w, r_{u c}}$ refer to the number of engines and landing gears. (The front and rear spars are assumed to have equal thickness, corresponding to the maximum calculated.)

The rib flanges are sized to support the control surfaces. Figure 3.20 illustrates the transfer of control surface loads to the ribs at the hinge-attachment points. The weight of the control surface $W_{c s}$ induces a torsional moment, which is reacted by the rib flange as a direct axial force $R_{c s}$. The rib-flange cross-sectional area is therefore

$$
A_{w, r_{f}}=\frac{R_{c s}}{\sigma_{u t s}} .
$$

The weight depends on the number of hinge attachment points that connect the control surfaces to the wing surface $N_{c s}$ (discussed further in the control surface sizing section below)

$$
W_{w, r_{f}}=2 \rho_{a l} g \sum_{j} N_{c s}^{(j)} A_{w, r_{f}} \bar{w}_{w, r_{f}}^{(j)}
$$

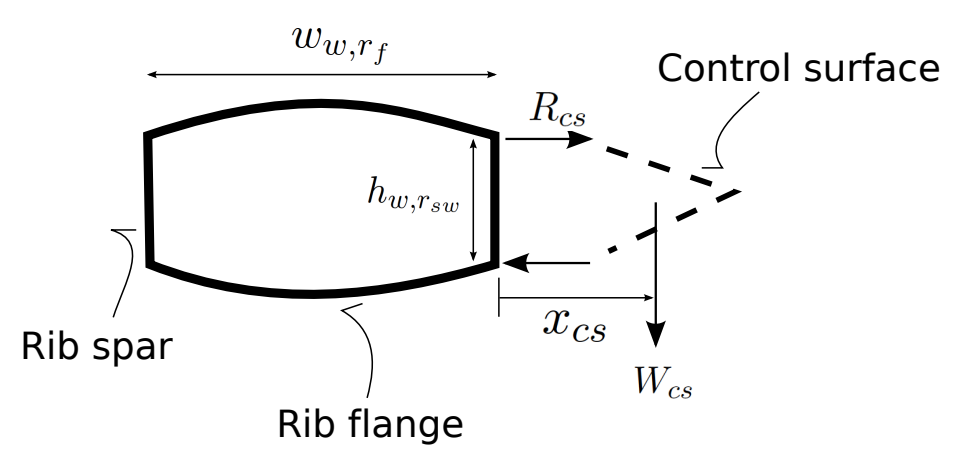

Figure 3.20: Schematic diagram of rib arrangement and forces at hinge attachment point.

\section{Control Surface}

For a conventional aircraft layout, the empirical correlations provided by Torenbeek (1976) are satisfactory to estimate the structural weight of flaps, slats, spoilers, ailerons, etc. In contrast, for the LFW, these items must be sized through a detailed structural analysis. Note, without 
the need for high-lift devices (see Chap. 2), only control surfaces are considered.

Each control surface is supported by two hinge attachment points. A spanwise separation of $55 \%$ along the control surface span is assumed. (Howe (2004) comments that tapering of the wing usually pushes the outer hinge further outboard, however this is neglected here). With a triangular cross section, as detailed in Fig. 3.21, the structural loads are reacted by a single I-beam spar boom.

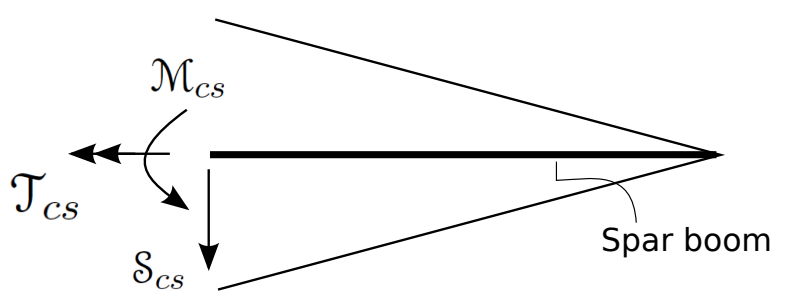

Figure 3.21: Schematic diagram of control surface structural arrangement and forces.

The shear force $\left(\mathcal{S}_{c s}\right)$ and bending moment $\left(\mathcal{M}_{c s}\right)$ acting at a hinge are calculated by integrating the pressure distribution and the moment of the pressure force, respectively, for a given surface deflection over, e.g, the last $10 \%$ of chord. An example pressure distribution is provided in Fig. 3.22.

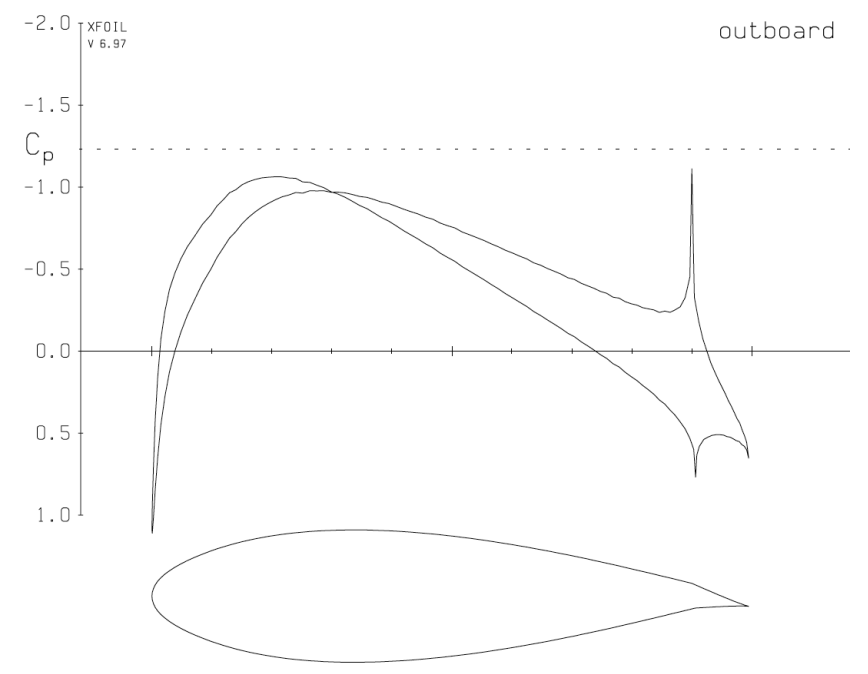

Figure 3.22: Outboard surface $C_{p}$ for a trailing edge flap deflection of $10^{\circ}$ over the last $10 \%$ of chord (output from XFoil).

The I-beam flanges are sized to react $\mathcal{M}_{c s}$. For simplicity, both the tensile and compres- 


\section{CHAPTER 3. DESIGN METHODOLOGY}

sive flanges are sized based on the stress limit corresponding to fatigue/crack propagation avoidance requirements. The shear web is sized to react vertical shear. (Torsional shear $\mathcal{T}_{c s}$ is neglected.) The total cross-sectional area, per hinge, is therefore

$$
A_{w, c s_{s b}}^{(j)}=\frac{\mathcal{M}_{c s}^{(j)}}{0.30 \sigma_{u t s}}+\frac{\mathcal{S}_{c s}^{(j)}}{0.5 \sigma_{u t s}}
$$

The total weight of the control surface spar booms, $W_{c s_{s b}}$, is then

$$
W_{c s_{s b}}=\rho_{a l} g \sum N_{c s_{s b}}^{(j)} k_{c s} \bar{c}_{\perp}^{(j)} A_{c s}^{(j)}
$$

where $k_{c s}$ is the proportion of chord dedicated to the control surfaces, and $N_{c s_{s b}}$ denotes the number of hinges over each spanwise region.

The cover skin thickness $t_{w, s_{c s}}$ is sized to withstand the distributed pressure forces. The cover skin weight is subsequently

$$
W_{c s_{s}}=\rho_{a l} g \sum_{j} 2 t_{w, s_{c s}}^{(j)} b^{(j)}\left(\left(\frac{h_{w, r_{s w}}^{(j)}}{2}\right)^{2}+\left(k_{c s} \bar{c}_{\perp}^{(j)}\right)^{2}\right)^{1 / 2}
$$

\section{Wing Structural Analysis}

The wing structure is treated as a root-cantilever beam. The shear force, bending moment and torsion couple are calculated along the spanwise reference axis by free-body analysis of the wing region outboard of the cross-section of interest. The structural loads are evaluated through numerical integration in the resolved coordinate system.

Figure 3.23 details the forces acting on panel faces ' $i$ ' and ' $i+1$ ', and the aerodynamic and inertial forces acting over a panel with area $A_{i}$. The aerodynamic force $\mathcal{L}_{i}$ is treated as a point load acting at quarter chord and is offset from axis ' $i$ ' by a distance $\Delta_{i} / 2=\left(y_{i+1}-y_{i}\right) / 2$; the resolved aerodynamic pitching moment contributions $\mathcal{M}_{\mathcal{T}_{i, c / 4}}^{r}$ and $\mathcal{T}_{\mathcal{T}_{i, c / 4}}^{r}$ also act here. The distributed inertial loads, e.g., baggage, payload, wing structural mass, etc are denoted by $\mathcal{W}$ (the combination of loads depends on which region of the wing is being analysed); they have a chordwise centre of gravity, $x_{i, c g_{\mathcal{W}}}$, and act a distance $\Delta_{i} / 2$ from panel edge $i$. (For the LFW, the fuselage structural mass also contributes to the distributed loading.) Point loads such as the engine, undercarriage, ground reaction, etc, are represented by $W_{P}$, and are assumed to act at the location of panel face $i$, at a chordwise location $x_{i, c g_{W_{P}}}$. 


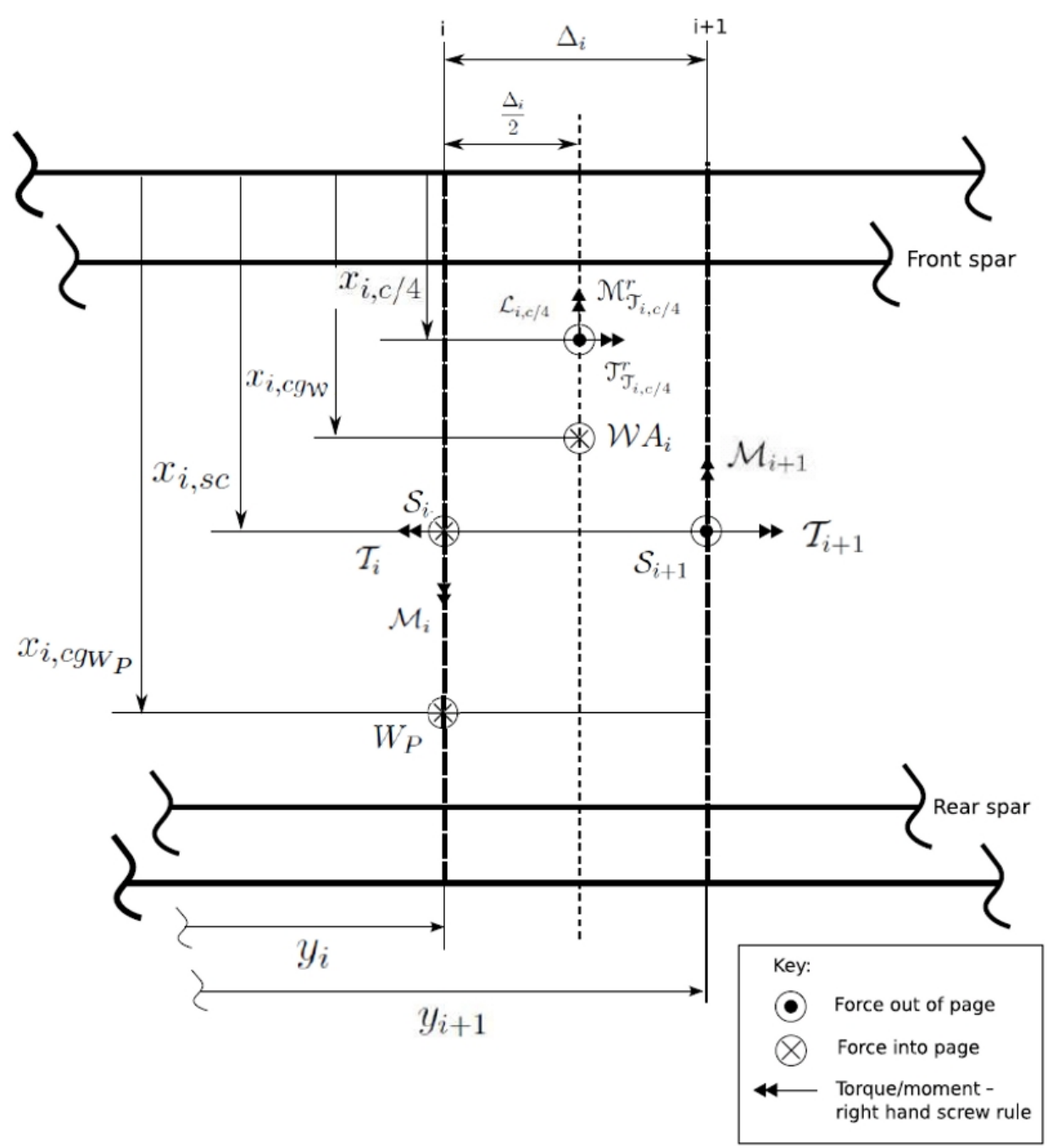

Figure 3.23: Structural force discretisation.

The shear force, bending moment and torque couple acting on the panel face $i$ are:

$$
\begin{aligned}
\mathcal{S}_{i}= & -\sum W_{P}-\mathcal{W} A_{i}+\mathcal{L}_{i, c / 4}+\mathcal{S}_{i+1} \\
\mathcal{M}_{i}= & \mathcal{M}_{i+1}+\mathcal{M}_{\mathcal{T}_{i, c / 4}^{r}}^{r}-\mathcal{L}_{i, c / 4} \times \frac{\Delta_{i}}{2}+\mathcal{W} A_{i} \times \frac{\Delta_{i}}{2}-\mathcal{S}_{i+1} \times \Delta_{i} \\
\mathcal{T}_{i}= & \mathcal{T}_{i+1}+\mathcal{T}_{\mathcal{T}_{i, c / 4}^{r}}^{r}+\mathcal{L}_{i, c / 4} \times\left(x_{i, s c}-x_{i, c / 4}\right)-\sum W_{P} \times\left(x_{i, s c}-x_{i, c g_{W_{P}}}\right) \\
& +\mathcal{W} A_{i} \times\left(x_{i, s c}-x_{i, c g \mathcal{W}}\right)
\end{aligned}
$$

The aerodynamic lift and pitching moment acting at the quarter-chord position are de- 
termined by AVL in the free-stream axes. The lift force requires no further consideration; however, the pitching moment contribution results in an additional bending moment

$$
\begin{aligned}
\mathcal{M}_{\mathcal{T}_{c / 4}}^{r} & =\mathcal{T}_{c / 4} \sin \Lambda \\
\mathcal{T}_{\mathcal{T}_{c / 4}}^{r} & =\mathcal{T}_{c / 4} \cos \Lambda .
\end{aligned}
$$

(For symmetrical aerofoil sections $C_{m 0}=0$, and hence $\mathcal{T}_{c / 4}$ is zero.)

Consider the moments and torques acting about the sweep discontinuity at station 2 shown in Fig. 3.24. The resolved moment and torque components $\mathcal{M}_{i+1}^{r}$ and $\mathcal{T}_{i+1}^{r}$, respectively, acting on the shaded element are given by

$$
\mathcal{M}_{i+1}^{r}=\mathcal{M}_{i+1} \cos \left(\Lambda_{2-3}-\Lambda_{1-2}\right)+\mathcal{T}_{i+1} \sin \left(\Lambda_{2-3}-\Lambda_{1-2}\right)
$$

and

$$
\mathcal{T}_{i+1}^{r}=\mathcal{M}_{i+1} \sin \left(\Lambda_{2-3}-\Lambda_{1-2}\right)+\mathcal{T}_{i+1} \cos \left(\Lambda_{2-3}-\Lambda_{1-2}\right)
$$

The actual moment at $i$ is the sum of the resolved component plus the additional moment that arises due to forces acting over the shaded region, $\mathcal{M}_{i}=\mathcal{M}_{i+1}^{r}+\ldots$. Consequently, the moment couple is slightly over-estimated. Since the error is a conservative one, further refinement is not attempted.

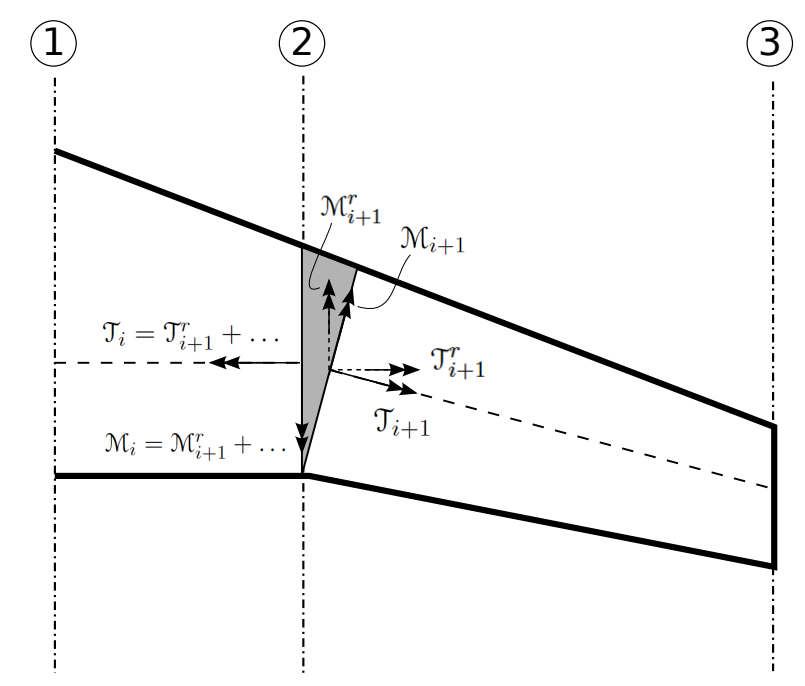

Figure 3.24: Resolution of moment and torque couples across a wing-sweep discontinuity. 


\subsubsection{Validity of Design Methodology}

In this section, the validity and accuracy of the design approach is investigated. A Boeing 737-200 aircraft ${ }^{1}$, for which a structural weight breakdown is provided by Kroo ${ }^{2}$, is sized.

The specified maximum takeoff weight is approximately $59 \mathrm{mt}$. Two Pratt \& Whitney JT8D turbofan engines weighing around $2.4 \mathrm{mt}$ each power the B737-200. For 136 passengers, the payload weight is $12,920 \mathrm{~kg}$. (The wing structural forces are evaluated for zero fuel weight.) The aircraft's economy cruise speed occurs at an altitude of $9145 \mathrm{~m}$ and flight Mach number 0.73 (Aboulafia (1996)).

\section{Geometry}

A simplified model of the aircraft is detailed schematically in Fig. 3.25. The fuselage is modelled as a cylinder with a semi-hemispherical nose endcap and tapered tailcone. The wings are low-mounted and have a constant quarter-chord sweep of $25^{\circ}$. The horizontal tailplane is mounted on the top-side of the fuselage with a quarter-chord sweep of $35^{\circ}$. Finally, the vertical fin has a quarter-chord sweep of $45^{\circ}$. The wing aerofoil cross-section is provided on the AVL website ${ }^{3}$; it has a maximum thickness-to-chord ratio of $11 \%$. Symmetrical NACA-4-digit aerofoils with a maximum thickness-to-chord ratio of $10 \%$ are assumed for the horizontal tailplane and vertical fin. The engines and undercarriage are assumed to act as point loads located on the leading edge at $40 \%$ half-span, and $75 \%$ chord at $30 \%$ half-span, respectively. The front and rear spars are assumed at $10 \%$ and $70 \%$ chord, respectively, for all surfaces.

\section{Wing and Tailplane Structures}

A typical 2.5-g pull-up manoeuvre in cruise is assumed critical for sizing of the wing structure. The structural forces that arise with and without self-weight included are shown in Fig. 3.26.

Overall, across the span, the shear force increases going inboards. There are two distinct drops in shear force at the engine and undercarriage positions. The, negative, increase in bending moment and slight kinks in the diagram may be attributed to the same factors as those for the shear force; however, there is a positive increase at the root due to the resolved torque component. The torque distribution shows a slight, overall, increase across the span; there is an increase at the engine position, and a decrease at that of the undercarriage; at the wing root, there is a sharp reduction due to the resolved moment component. This is

\footnotetext{
${ }^{1}$ www.aerospaceweb.org, September 2011 .

${ }^{2}$ http://adg.stanford.edu/aa241/AircraftDesign.html

${ }^{3}$ http://web.mit.edu/drela/Public/web/avl/
} 


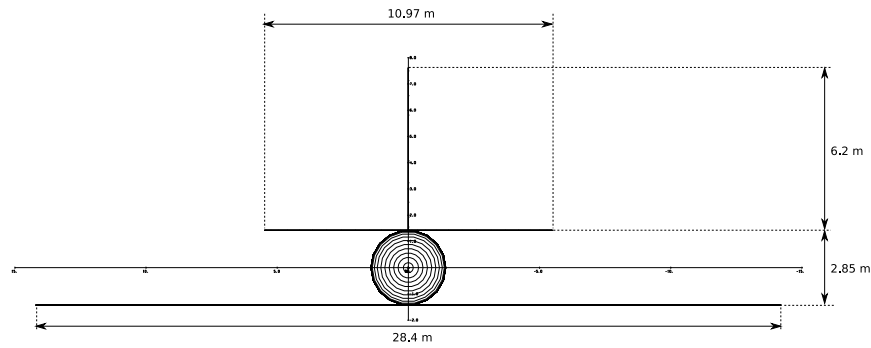

(a) Front view.

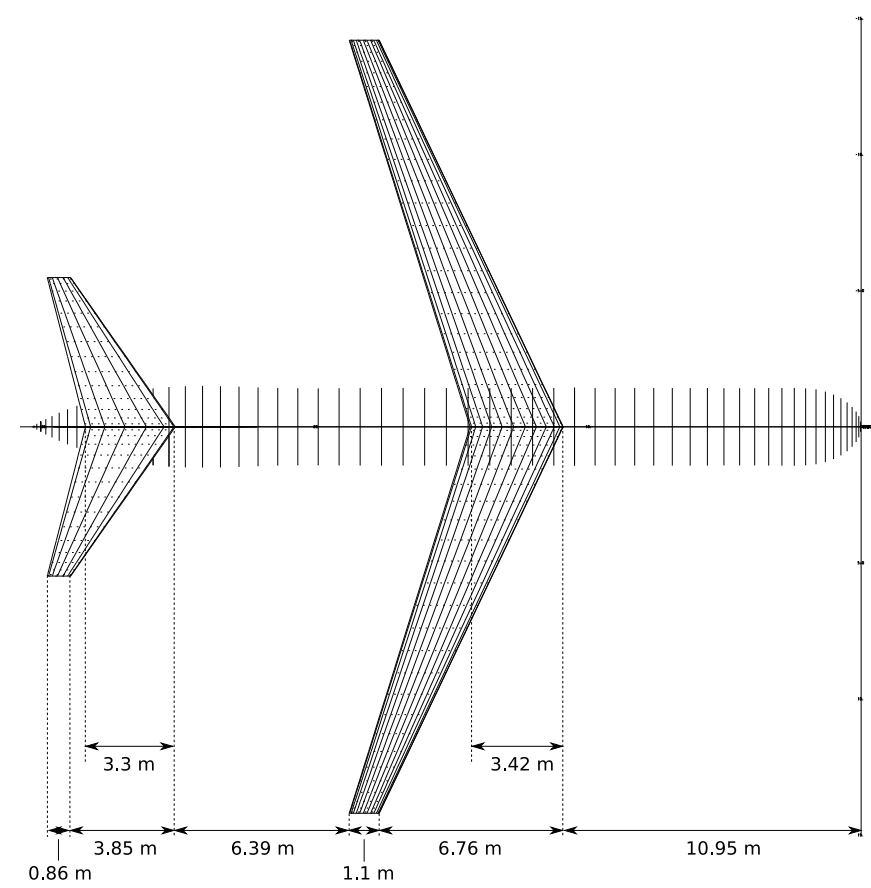

(b) Plan view.

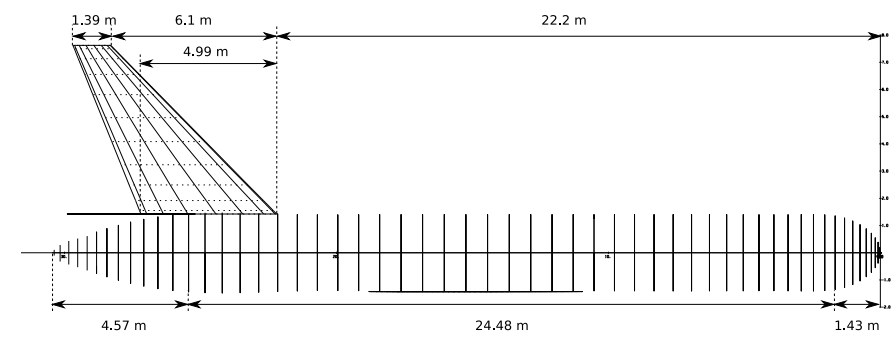

(c) Side view.

Figure 3.25: Assumed Boeing 737-200 configuration. 
physically consistent as the wing is swept back, and therefore the overall lift lies aft of the shear centre; whilst locally, the lift force acts forward of the shear centre. The inclusion of self-weight acts to provide some bending relief, but does not affect the torque distribution, as self-weight acts at the shear centre.
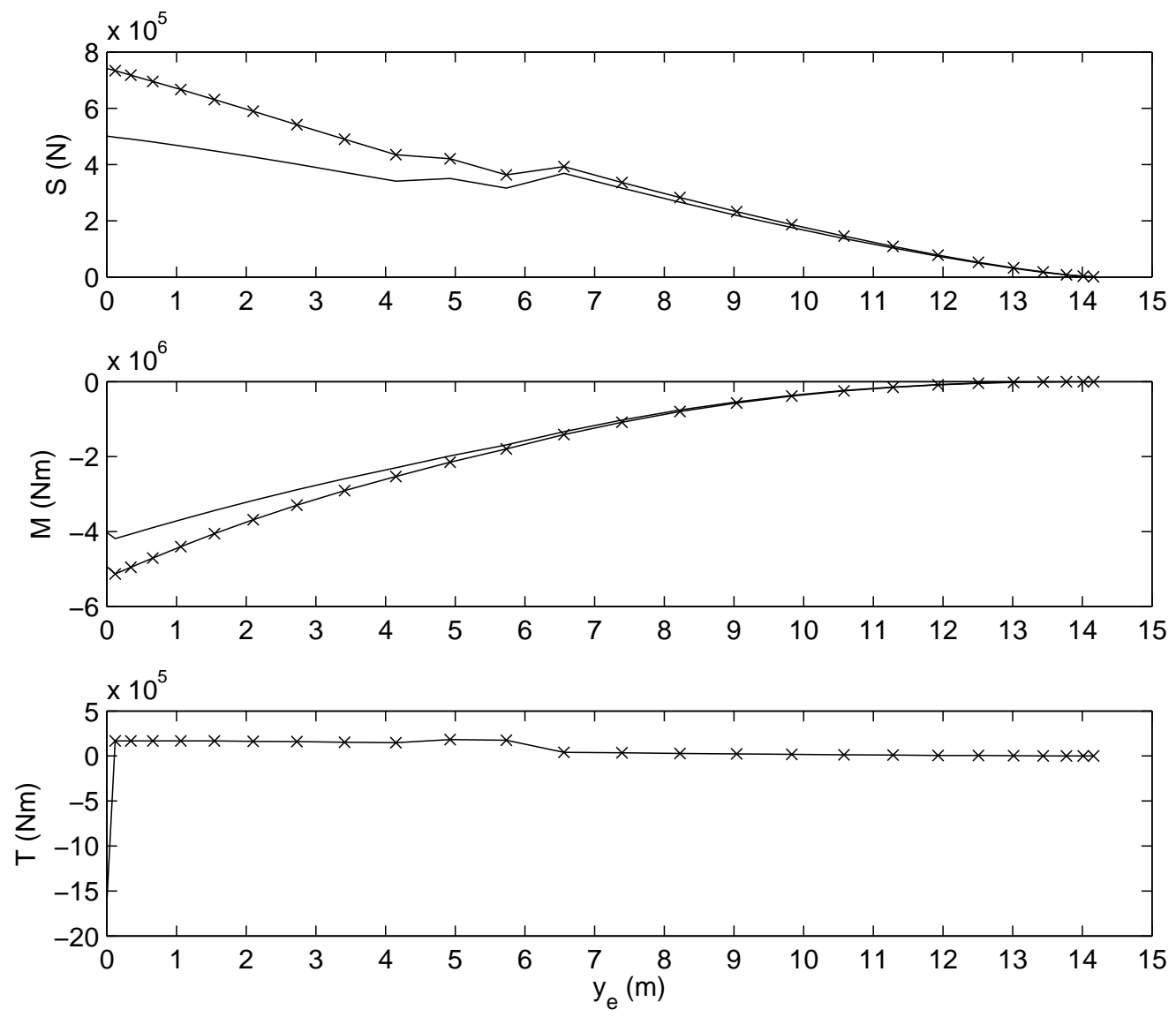

Figure 3.26: Wing shear force, bending moment and torque diagrams - initial estimate (solid x-marked line) and final estimate (solid line).

Howe (2004) notes that, typically, a pull-out manoeuvre in a nose dive is critical for the horizontal stabiliser; here, the lift distribution on the tailplane in trimmed cruise is scaled to a maximum section $C_{l}$ of 0.80 (Polentz (1949)). The vertical fin is sized to react loads incurred during engine-out at takeoff and is scaled by a factor of 0.70 to account for inertial effects (Greitzer et al. (2010)); the resulting yawing moment coefficient $C_{n}$ is assumed to be 0.002; rudders are taken to occupy the full fin height beginning at $70 \%$ chord. 


\section{CHAPTER 3. DESIGN METHODOLOGY}

The estimated wing structural weight breakdown is provided in Tab. 3.3. The total, structural, weight of the tailplane is estimated to be $1,052 \mathrm{~kg}$.

Table 3.3: Estimated wing structural weight breakdown for the B737-200.

\begin{tabular}{lcc}
\hline \hline Component & Weight $(\mathrm{kg})$ & Percentage (\%) \\
\hline Skin and stringers & 5,137 & 89 \\
Spar webs & 129 & 2 \\
Flaps, slats, spoiler and control surfaces & 415 & 7 \\
Ribs & 97 & 2 \\
\hline Total & 5,778 & 100 \\
\hline \hline
\end{tabular}

\section{Fuselage Structure}

A breakdown of the fuselage weight is given in Tab. 3.4. The estimate here is 1,789 kg, which is significantly lower than that quoted for the B737-200 (see Tab. 3.5).

Table 3.4: Estimated fuselage structural weight breakdown for the B737-200.

\begin{tabular}{lcc}
\hline \hline Component & Weight $(\mathrm{kg})$ & Percentage (\%) \\
\hline Shell & 971 & 60 \\
Tail cone & 6 & $\sim 0$ \\
Additional side material & 8 & $\sim 0$ \\
Additional vertical material & 0 & 0 \\
Windows & 251 & 20 \\
Insulation & 153 & 10 \\
Floor & 400 & 30 \\
\hline Total & 1,789 & 100 \\
\hline \hline
\end{tabular}

Torenbeek (1976) presents a basic weight penalty method for fuselage structural weight prediction, which is empirically based. (Hence this is not applicable to a multi-bubble shell.) Aspects that have been neglected in this study thus far include:

- gross shell modifications such as doors, fasteners, rivets, etc;

- support structures for the wing/fuselage connection, tailplane, and nose landing gear;

- wing/fuselage fairings.

The first item is typically a factor of two above the gross shell weight, adding $1789 \mathrm{~kg}$. For low-wing aircraft with a continuous wing box, the wing/fuselage connection weight is $100 \mathrm{~kg}$. 
The tailplane support structure adds $145 \mathrm{~kg}$. The nose landing gear bay weight adds $166 \mathrm{~kg}$. Finally, wing/fuselage fairings add $300 \mathrm{~kg}$. Therefore, the total added weight is estimated to be around $2355 \mathrm{~kg}$.

\section{Structural Weight Breakdown}

A breakdown of the estimated structural weight is summarised in Tab. 3.5, and compared against those provided by Kroo ${ }^{1}$. The wing and tailplane weights compare well, with a percentage difference of $14 \%$ and $2 \%$, respectively. The fuselage weight is $23 \%$ lower. The total structural weight compares well, with a difference of just 5\%. Given the approximate nature of the calculated loadings and assumed geometries, the structural weight predictions are deemed sufficiently close enough to support the structural design approach used here.

Table 3.5: Structural weight breakdown for the B737-200.

\begin{tabular}{lcc}
\hline \hline Component & Weight $(\mathrm{kg})$ & Actual $(\mathrm{kg})$ \\
\hline Fuselage & 4,144 & 5,412 \\
Wing & 5,778 & 5,068 \\
Tailplane & 1,052 & 1,074 \\
\hline Total & 10,974 & 11,554 \\
\hline \hline
\end{tabular}

\section{Operating Empty Weight Breakdown}

The aircraft operating empty weight is created from estimates of structures, propulsion system, landing gear and fixed equipment. A breakdown is provided in Tab. 3.6.

The structures weight compares very well. The landing gear weight estimate is conservative, with a difference of $19 \%$. The calculated fixed equipment weight is $57 \%$ greater. The total estimated OEW is $16 \%$ greater, and is due mainly to the large difference in fixed equipment weight.

\footnotetext{
${ }^{1}$ http://adg.stanford.edu/aa241/AircraftDesign.html
} 
Table 3.6: Operating empty weight breakdown for the B737-200.

\begin{tabular}{lcc}
\hline \hline Component & Weight $(\mathrm{kg})$ & Actual $(\mathrm{kg})$ \\
\hline Structure & 10,974 & 11,554 \\
Landing gear & 2,187 & 1,833 \\
Propulsion & 4,289 & 4,289 \\
Fixed equipment & 12,920 & 8,220 \\
\hline Total & 30,370 & 26,083 \\
\hline \hline
\end{tabular}

\subsubsection{Mission Analysis}

The mission fuel consists of climb fuel and fuel needed to complete the mission. In addition, the aircraft must carry reserve fuel in case of a diversion and make allowances for unusable fuel.

The climb fuel is calculated using Eq. 3.83:

$$
m_{f}^{c l i m b}=\frac{\Delta(K E+P E)}{\eta_{o v} L C V},
$$

where $\Delta(K E+P E)$ is the change in kinetic and potential energies between takeoff and maximum altitude, and $\eta_{o v}$ is the overall engine efficiency: in cruise with suction for the LFW, and at top-of-climb for the competitor aircraft.

The fuel to complete the mission is calculated by applying the Breguet range equation over the entire distance (including climb and descent)

$$
s=-\frac{V L / D}{g \cdot s f c} \log \left(\frac{W_{e}}{W_{s}}\right) .
$$

For the LFW this must be broken down into climb/landing and cruise, as LFC is only assumed available once the aircraft has reached an altitude above 15,000 ft. (The average climb and descent angles are taken as $2^{\circ}$.) Therefore, cruise (without suction) values for lift-to-drag ratio, velocity, and $s f c$ are assumed over the climb and landing flight phases up to the 'suction initiation' altitude.

The reserve fuel must be sufficient to ensure, if necessary, the aircraft can fly $200 \mathrm{~nm}$ and hold for a further $0.75 \mathrm{hrs}$ at the cruise fuel-burn rate in the event of a diversion (Hileman et al. (2007)). In the event of a suction system failure, it is assumed that the LFW will be allowed to land immediately. Therefore, the more critical of the two methods sets the reserve fuel weight for the LFW. The unusable fuel is taken as $1 \%$ of the sum of mission and reserve fuel. 


\section{Chapter 4}

\section{Boundary-Layer Suction System}

\section{DESIGN}

The aim of this chapter is to provide insight into the parameters affecting practical LFC suction power requirements for a commercial LFW transport aircraft. Specifically, the objectives are: to characterise the increased power requirement due to the individual system elements, relative to the theoretical minimum associated with the unavoidable boundary-layer losses; to investigate the trade-off between reduction of the additional power requirement and increasing system complexity; and to formulate approximate calculation methods that may be of use in overall LFW design and optimisation. The chapter begins by introducing the boundary-layer stability code, which calculates the 'optimal' distribution of suction for the maintenance of laminar flow. Then, the influences of a number of key design parameters on the level of suction are investigated. Finally, the main contributors to the various pressure drops through the suction system are discussed in turn.

\subsection{Boundary-Layer Suction Calculation Method}

In developing a suction system design, the required level and distribution of suction for the maintenance of laminar flow must first be determined. In this section a boundary-layer solver is implemented and validated, and a suction algorithm is developed and compared against an example calculation by Head (1959).

\subsubsection{Boundary Layer Solver}

The current industry standard for transition prediction is the $e^{N}$-method, which is based on the growth rate of linear instabilities in the boundary-layer flow and empirically determined 


\section{CHAPTER 4. BOUNDARY-LAYER SUCTION SYSTEM DESIGN}

criteria for the degree of growth necessary for transition to occur (Joslin (1998b)). In general, there are several classes of instability that cause transition. However, restricting the analysis here to wing-sweep angles below about $25^{\circ}$, attention can be restricted to the influence of two-dimensional Tollmien-Schlichting instabilities (see Chap. 2). In this case simpler methods, which are more computationally efficient, may be employed; here the Eppler and Somers (1980) two-dimensional semi-empirical computational model, shown to give excellent predictions of transition in wind tunnel experiments (Bowers and Sim (1984)), is employed.

The computational analysis is divided into inviscid and viscous steps. The aerofoil surface is discretised into a number of panels. The inviscid calculation is performed using XFoil, which calculates the aerofoil surface-pressure distribution via an incompressible vortex-panel method combined with a compressibility correction (see Chap. 3). (The default number of panels in XFoil is 160, with a maximum of 354. The accuracy in the final calculated suction quantity was found to worsen with increases in maximum section thickness and free-stream Mach number; $C_{l}$ variation had no effect. For a NACA0030 aerofoil, with $M_{\infty}=0.5$ and $C_{l}=0$, there is an error of $1.3 \%$ between the default and maximum number of panels.) The viscous analysis takes the pressure distribution and, for a specified chord Reynolds number, calculates the development of the boundary layer using the Eppler and Somers (1980) method.

The Eppler and Somers (1980) method is based on a numerical solution of the (incompressible) integral momentum and energy equations:

$$
\delta_{2}^{\prime}+\left(2+H_{12}\right) \frac{u_{e}^{\prime}}{u_{e}} \delta_{2}=C_{f}-\frac{V_{0}}{u_{e}}
$$

and

$$
\delta_{3}^{\prime}+3 \frac{u_{e}^{\prime}}{u_{e}} \delta_{3}=C_{d}-\frac{V_{0}}{u_{e}}
$$

respectively, where: $C_{f}=\epsilon^{*} / R e_{c} \frac{u_{e}}{U_{\infty}} \frac{\delta_{2}}{c} ; C_{d}=2 D^{*} / R e_{c} \frac{u_{e}}{U_{\infty}} \frac{\delta_{2}}{c} ;$ and $\epsilon^{*}, \mathrm{D}^{*}, H_{12}$ are functions of the energy shape factor $H_{32}$.

The location of transition is based on a critical momentum thickness, which is a function of $H_{32}$ only:

$$
\ln \left(\operatorname{Re}_{\delta_{2}}\right)=18.4 H_{32}-21.78
$$

Previous boundary-layer stability studies - using the $e^{N}$-method - have found that, in the case of excessive suction, the critical amplification value $(\mathrm{N})$ for the onset of transition reduces i.e. the transition criteria for sucked and un-sucked flows are not identical (Ellis et al. (2000)). Eppler (1978) comments that, because suction is normally combined with finite disturbances, transition mostly occurs before the linear stability limit is reached. For $H_{32}>1.62$, which can only be reached by suction, the Eppler and Somers (1980) empirical 


\subsection{BOUNDARY-LAYER SUCTION CALCULATION METHOD}

criterion predicts transition well before that corresponding to the linear stability limit.

At low local Mach numbers, the influence of compressibility on the stability of the boundary layer may be taken as negligible; in fact, Berry (1989) points out that the well established Orr-Sommerfeld equation has this as an underlying assumption. For subcritical flow regimes, compressibility effects become increasingly important with Mach number, with the growth rate of the Tollmien-Schlichting type instability reducing as a consequence. This highly stabilising influence was exploited by Viken and Wagner (1991) in the design of high-speed natural-laminar-flow aerofoils. Mack (1979), through a linear stability analysis, found that incompressible codes over-predict the local amplification rates. Harris et al. (1988) performed experimental investigations on a swept-supercritical aerofoil employing LFC and calculated that, relative to incompressible predictions, compressibility effects reduced the amplification values in the mid-chord Tollmien-Schlichting wave dominated regions by approximately $40 \%$. Harris et al. (1988) chose to base their final suction-system design on the incompressible calculations, due to the increased conservatism of the estimated suction requirements that follow. Further studies on the influence of compressibility can be found in the works of Atkin (2004), Bushnell and Malik (1987) and Berry et al. (1987). However, the literature does not provide a quantitative indication of the likely error in incompressible LFC suction calculations.

Nonetheless, the simplicity and conservatism of an approach employing the incompressible form of the integral equations make it attractive. Furthermore, surface roughness effects (see Joslin (1998b)) are neglected in the present study, and a conservative calculation goes some way in compensating for this. The integral boundary-layer equations are solved using a standard 4th-order Runge-Kutta algorithm with adaptive spacing, with the relative error tolerance set to $0.1 \%$.

The laminar-boundary-layer solver was first validated by modelling a zero-pressure-gradient flow over a flat plate: the $\delta_{2}$ variation was in agreement with Blasius's prediction (Young (1989)). Next, assuming a linearly retarded velocity profile, the code's ability to predict laminar separation was confirmed against Howarth's boundary-layer calculation (Young (1989)). Further checks, for pressure distributions corresponding to a range of symmetric aerofoil thicknesses, lift coefficients and chord-based Reynolds numbers, were made against Thwaites' method (Young (1989)) with excellent results.

Over certain regions of the aerofoil, suction may not be permissible. Therefore it is also necessary to solve Eqs. 4.1 and 4.2 for a turbulent boundary layer. The only differences that arise are the forms of $C_{f}, C_{d}$ and the empirical relations depending on $H_{32}$. Turbulent separation is flagged when $H_{32}$ drops below 1.46. 


\section{CHAPTER 4. BOUNDARY-LAYER SUCTION SYSTEM DESIGN}

\subsubsection{Suction Algorithm and Validation}

A calculation method is required such that the power demanded to maintain laminar flow is kept to a minimum. Head (1959) claimed that applying just enough suction to keep the boundary layer in a condition of neutral stability would give a total power requirement which approximates closely to that minimum. While not immediately evident, this claim can be supported by a simple argument. On consideration of the minimum (incompressible) pump power per unit span required to return the sucked flow to the free-stream stagnation pressure:

$$
\int_{0}^{c}\left[P_{0, \infty}-P_{w}\right] V_{0} d x
$$

and assuming, as an approximation, that suction is only necessary in regions of adverse pressure gradient, it is apparent that suction earlier, where $P_{w}$ is lower, is penalised more than suction later on. Therefore, in determining $V_{0}$ the minimum allowable value is aimed for, i.e., to maintain the boundary layer on the verge of transition. This can be achieved via the following algorithm.

Consider the integral momentum and energy equations, Eqs. 4.1 and 4.2: assuming $C_{f}$ and $C_{d}$ are not significantly affected by the application of surface suction, the energy shape factor at the end of a panel of length $\Delta s$ may be estimated as:

$$
H_{32}=\frac{\delta_{3}^{(i+1)}-\left(V_{0} / u_{e}\right) \Delta s}{\delta_{2}^{(i+1)}-\left(V_{0} / u_{e}\right) \Delta s},
$$

where $i+1$ denotes the conditions at the end of the panel which would apply in the absence of suction. To maintain the boundary layer on the verge of transition, $V_{0}$ should be set such that the stability criterion given by Eq. 4.3 is not flagged; a target energy shape factor $H_{32}^{(t)}$ is thereby defined and set equal to the right-hand-side of Eq. 4.4. On rearrangement, an initial estimate of the suction velocity required is obtained:

$$
\frac{V_{0}}{u_{e}}=\frac{\left(H_{32}^{(t)}-H_{32}^{(i+1)}\right) \delta_{2}^{(i+1)}}{\left(H_{32}^{(t)}-1\right) \Delta s} .
$$

The initial suction estimate may deviate significantly from the minimum required, especially in regions where $C_{p}$ changes considerably. Therefore, the calculation is then iterated to find the 'optimised' panel suction corresponding to Head's criterion.

To assess the accuracy of the algorithm, the distribution of suction calculated for a NACA $65_{4}-021$ aerofoil at zero incidence (with an incompressible surface velocity distribution as 
shown in Fig. 4.1) and for a $R e_{c}$ of 20 million, is compared against the prediction of Head (1959) in Fig. 4.2. NACA 6-series aerofoils were designed to maintain natural laminar flow over a large percentage of chord by delaying the adverse pressure gradient. Ladson and Brooks (1974) explain that the thickness envelope was developed using aerofoil theory; however, no simple definition of the shape is available. The aerofoil required here was generated using the Ladson and Brooks (1974) computational program, which provides a reasonable approximation to the thickness distribution. The corresponding velocity distribution was then generated using XFoil. It agrees closely with that given by Head (1959) (also shown in Fig. 4.1).

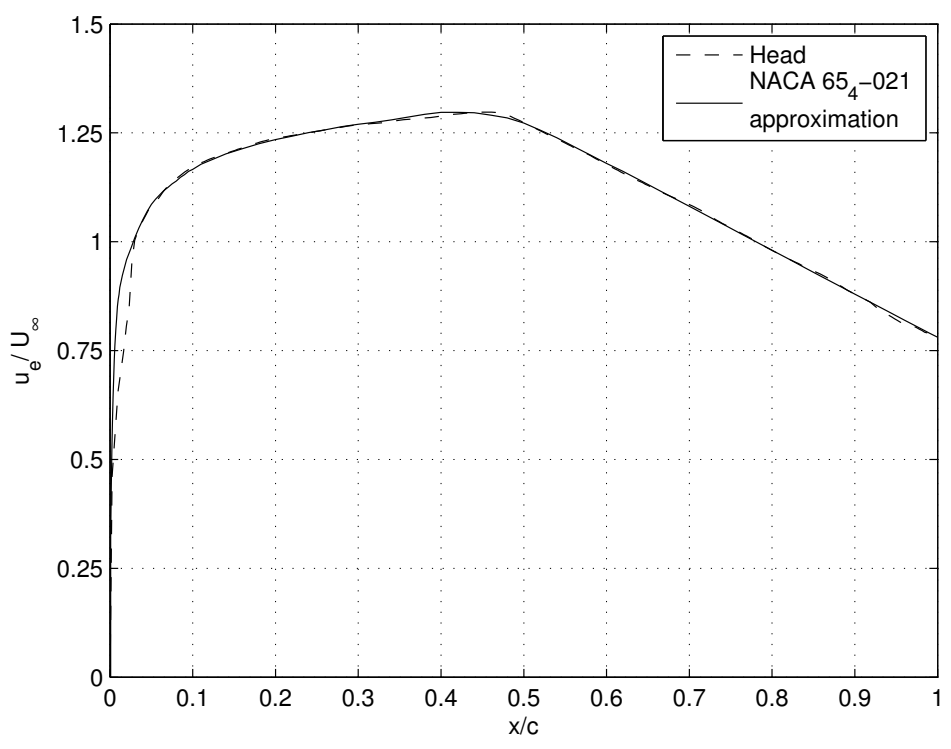

Figure 4.1: NACA $65_{4}-021$ incompressible surface velocity distribution $-C_{l}=0$.

The calculated level of distributed suction compares very well with Head's prediction downstream of $40 \%$ chord. The obvious difference between the two distributions is the chordwise position at which suction is initiated; this is due to the difference between Lin's transition criterion (which Head uses in conjunction with the Thwaites solution for a laminar boundary layer) and that of Eppler and Somers (1980). Both predict transition within the weaklyfavourable pressure-gradient region between 10\% and $45 \%$ chord (as might be expected for such a high Reynolds number), but Lin's does so earlier. Head estimates (for the upper and lower surface combined) $C_{Q}=5.14 \times 10^{-4}$; here, it is calculated that $C_{Q}=5.18 \times 10^{-4}-$ the discrepancy, in terms of volumetric flow-rate, is negligible.

A final comparison is made against Head's calculated momentum thickness distribution 
in Fig. 4.3. There is excellent agreement between the two methods.

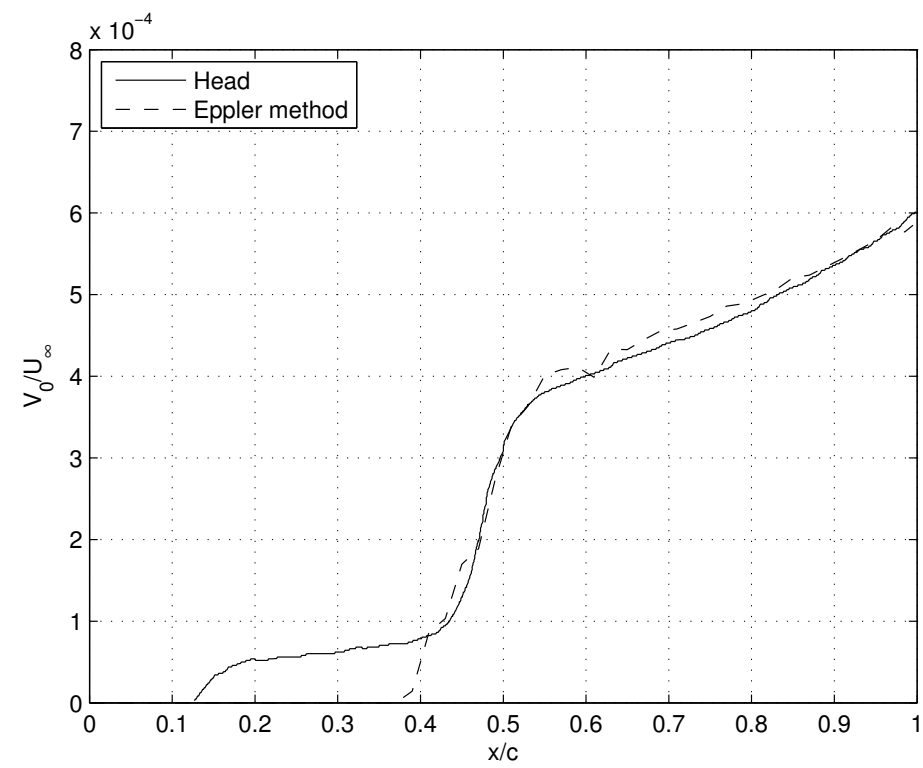

Figure 4.2: Comparison of calculated suction distribution - NACA $65_{4}-021$ aerofoil, $C_{l}=0$ and $R e_{c}=20 \times 10^{6}$.

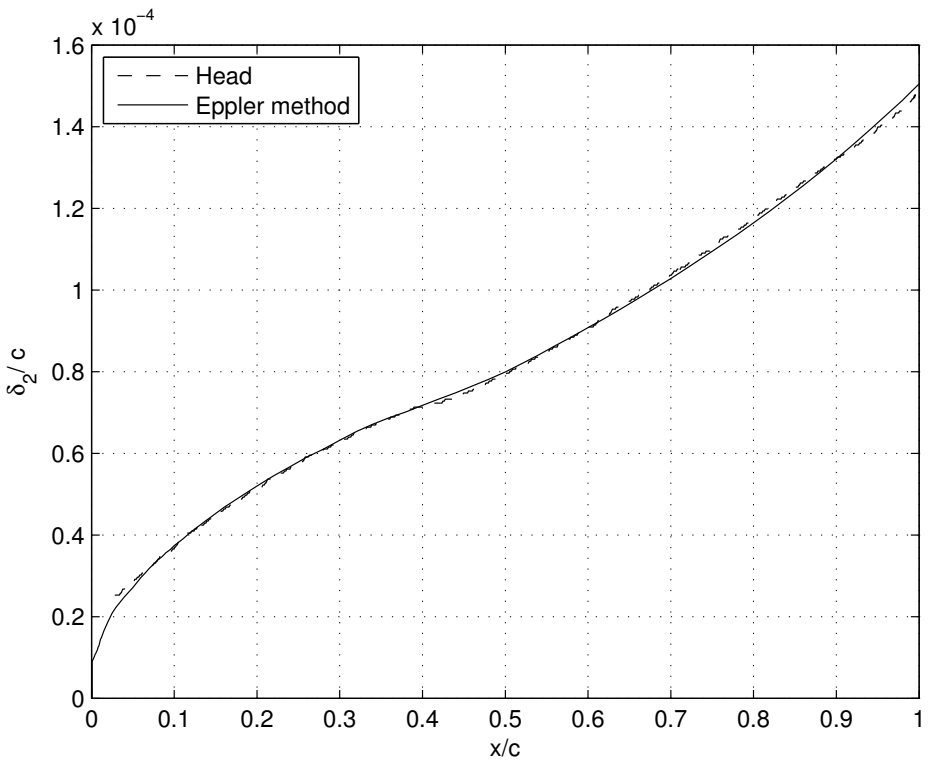

Figure 4.3: Comparison of calculated momentum thickness distribution - NACA $65_{4}-021$ aerofoil, $C_{l}=0$ and $R e_{c}=20 \times 10^{6}$. 


\subsection{Pump Power Analysis}

With the method of calculating the desired pattern of inflow to maintain laminar flow determined, it remains to estimate the power input necessary to drive the suction system. In this section, a non-dimensional power coefficient is defined.

In addition to the stagnation pressure loss in the boundary layer, there is an associated drop across each of the hardware components (detailed later in Fig. 4.11). The suction pumps are required to raise the stagnation pressure of the sucked flow back to a value that allows it to be discharged with some speed V at free-stream static pressure. Assuming adiabatic and reversible flow across the pump, an area-distributed pump-power coefficient $\tilde{w}_{p}$ is:

$$
\tilde{w}_{p}=\frac{\rho V_{0}(x, z) c_{p} T_{0, i n}\left(P P R^{\frac{\gamma-1}{\gamma}}-1\right)}{\frac{1}{2} \rho_{\infty} U_{\infty}^{2} \cdot U_{\infty}},
$$

where the pump pressure ratio $P P R=P_{0, e x} / P_{0, i n}(x, z)$. The total pump power coefficient $\widetilde{W}_{p}$ is subsequently:

$$
\widetilde{W}_{p}=\frac{1}{b c} \int_{0}^{b} \int_{0}^{c} \tilde{w}_{p} d x d z
$$

(The coordinate axes ' $x$ ' and ' $z$ ' are defined in Fig. 4.11.) The principal variables affecting these dimensionless coefficients are the distribution of suction flow rate $\left(V_{0}\right)$ and the increase in total pressure (PPR) required across the pumps. The latter depends not only on the wing pressure distribution, porous skin and ducting network, but also on the discharge velocity of the sucked flow.

The works of Rioual et al. (1996), Head (1955) and Edwards (1961) have shown that, based on a consideration of the total power required to overcome drag forces, it is optimal to discharge at a velocity slightly above free-stream velocity, depending on the relative efficiencies of the suction pump and aircraft propulsion systems. However, the benefit of the excess is typically small. Furthermore, Atkin (2004) comments that the effect of losses in the system is to flatten the minimum in the pump-drag versus exhaust Mach number relationship. Finally, given that the engine sizing for an LFW aircraft is likely to be determined by take-off requirements (see Chap. 2), increasing the discharge velocity above free-stream could increase suction-pump weights without reducing propulsion system weight; therefore, a free-stream exit velocity is unlikely to be far from optimal. In the absence of propulsion system information it is assumed that $V=U_{\infty}$, and hence $P_{0, e x}=P_{0, \infty}$. 


\subsection{Minimum Suction Requirements}

Once the wing geometry and flight condition are set, the minimum suction and power requirements can be determined independently of any details of the LFC suction system design. The aim of this section is to provide estimates of these requirements for the range of design parameters expected of a LFW aircraft, and to understand which parameters have the greatest influence.

The LFW is likely to require a high aspect-ratio wing and low $C_{l}$. When combined with a section height sufficient for passenger accommodation, and structural considerations, this gives rise to a thick wing with low sweep. To keep the flow subcritical requires a low cruise Mach number. Typical design parameter values are: $R e_{c}=40 \times 10^{6}, t / c=28 \%$, $C_{l}=0.15$ and $M_{\infty}=0.56$. As the section geometry will be significantly constrained by non-aerodynamic considerations, NACA00 series aerofoils are selected for this study. First, the suction requirements for the parameter values given above are characterised, and then the variation with $C_{l}, R e_{c}, M_{\infty}$ and maximum $t / c$ is investigated.

\subsubsection{Typical Suction and Power Distributions}

The upper-surface $C_{p}$ distribution for a NACA0028 aerofoil at the chosen design point is plotted in Fig. 4.4, along with the 'optimised' distribution of suction required for laminarisation. The suction requirement starts just upstream of the suction peak and remains reasonably constant until the severe adverse-pressure-gradient region over the last $10 \%$ of chord. The suction coefficient for the upper surface is $C_{Q}=3.78 \times 10^{-4}$.

The loss in stagnation pressure associated with the boundary-layer flow is given by $\Delta P_{B L}=P_{0, \infty}-P_{e}$ (based on Prandtl's assumption that the pressure across the boundary layer is constant (Young (1989))), and is plotted in dimensionless form in Fig. 4.5, which also repeats the suction velocity distribution. The (chordwise-distributed) minimum power requirement is approximately the product of these two quantities. However, for the exact minimum power calculation, Eqs. 4.6 and 4.7 are used. Here $P_{0, i n}=P_{e}, P_{0, e x}=P_{0, \infty}$ and, assuming adiabatic flow in the boundary layer, $T_{0, i n}=T_{0, \infty}$.

As discussed in Sec. 4.1, the greatest stagnation pressure loss in the boundary layer is concentrated in regions of low pressure (for example, near the suction peak); consequently, although the sucked velocity is low in comparison to that toward the trailing edge, it is penalised heavily - this is evident from the distribution of $\tilde{w}_{p}$ in Fig. 4.6. The total pump power coefficient for the upper surface is $\widetilde{W}_{p}=4.11 \times 10^{-4}$. At the other extreme from this idealised minimum, one could envisage a suction system in which all the sucked flow enters a single chamber, with the pressure maintained just below the value of $P_{e}$ at the suction 


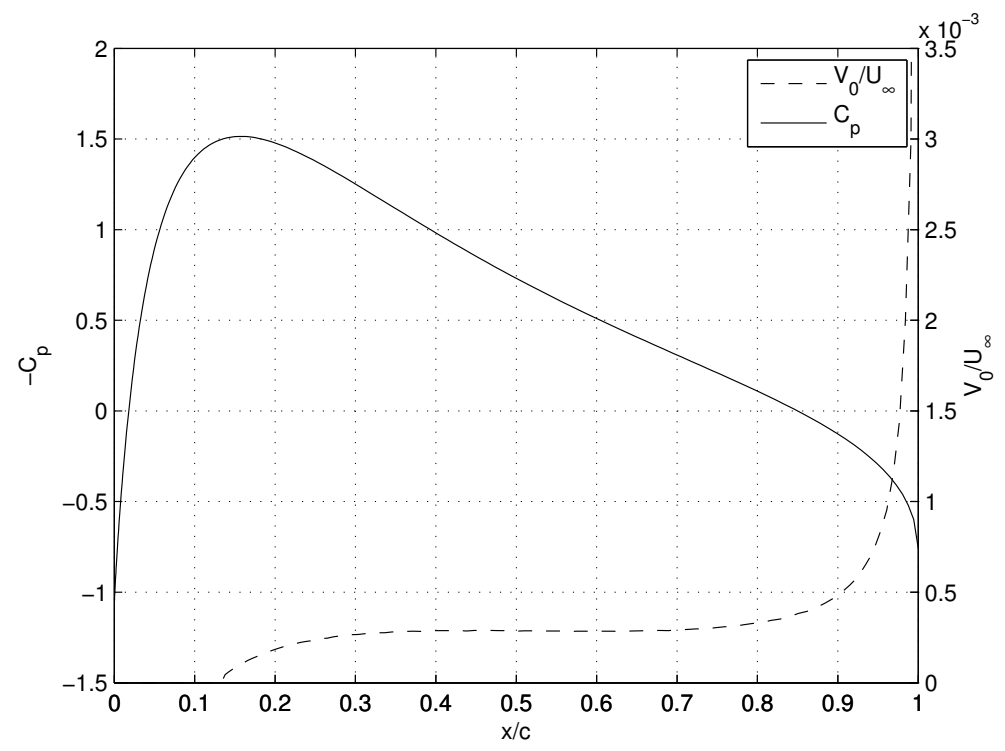

Figure 4.4: Section pressure (solid) and suction velocity distribution (dashed) for the upper surface of a NACA0028 aerofoil $-M_{\infty}=0.56, R e_{c}=40 \times 10^{6}$ and $C_{l}=0.15$.

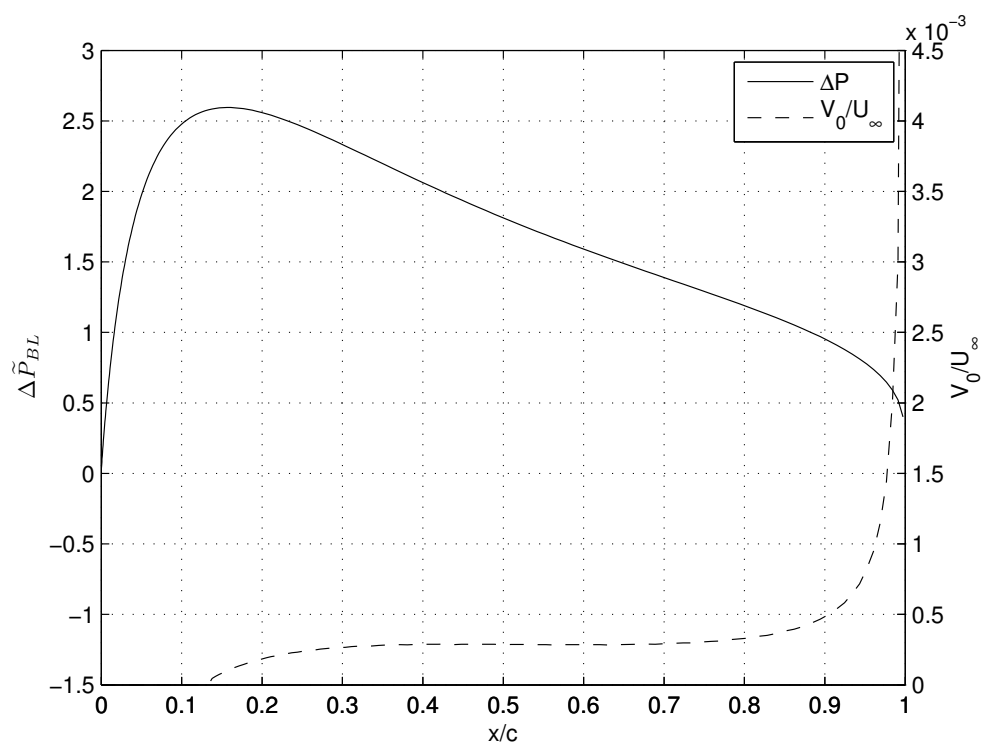

Figure 4.5: Distribution of dynamic pressure loss through boundary layer (solid), and suction velocity (dashed) for the upper surface of a NACA0028 aerofoil $-M_{\infty}=0.56, R e_{c}=40 \times 10^{6}$ and $C_{l}=0.15$. 


\section{CHAPTER 4. BOUNDARY-LAYER SUCTION SYSTEM DESIGN}

peak, and with the suction velocity distribution achieved by varying the skin resistance. This is the basis of the power requirement estimate of Pearce (1983); for this case it yields $\widetilde{W}_{p}=11.0 \times 10^{-4}$, almost three times greater than the minimum.

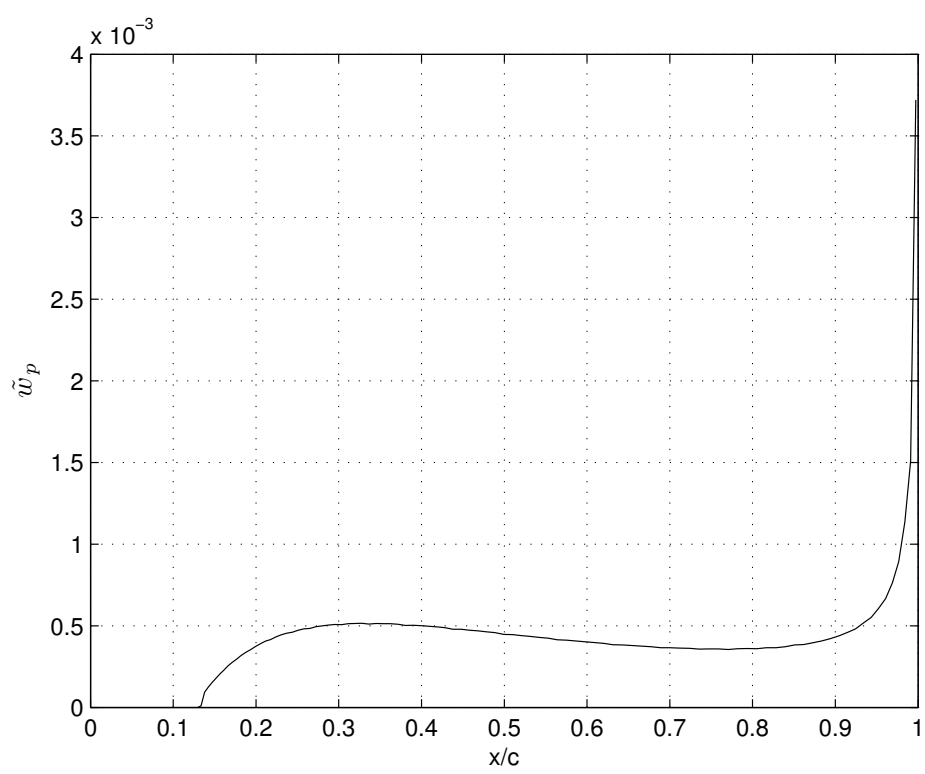

Figure 4.6: Distribution of (area-distributed) power to overcome losses through boundary layer.

\subsubsection{Parameter Study}

The dependence of the overall suction coefficient (upper and lower surface combined) on chord Reynolds number is well established as $C_{Q} \propto R e_{c}^{-1 / 2}$ (see, for example, Pankhurst and Gregory (1952)); this result is reproduced in Fig. 4.7 using the suction algorithm developed for the present study. In Eq. 4.6, the power requirement depends directly on the distribution of $V_{0}$ and the pressure drop. The integrated area of $V_{0} \sqrt{R e_{c}} / U_{\infty}$ along the chord, i.e. $C_{Q} \sqrt{R e_{c}}$, is almost exactly independent of $R e_{c}$, as expected. However, as chord Reynolds number is increased, the suction initiation point moves further upstream, and the mean level of $V_{0} \sqrt{R e_{c}} / U_{\infty}$ over the suction region drops. Sucked flow near the surface pressure minimum is penalised more in terms of power, so this redistribution leads to the gradual increase in $\widetilde{W}_{p} \sqrt{R e_{c}}$ with $R e_{c}$ observed in Fig. 4.7 .

The influence of $t / c$ on suction is detailed in Fig. 4.8, for three NACA00 series aerofoils with maximum thickness-to-chord ratios of $10 \%, 20 \%$ and $30 \%$; the corresponding $C_{p}$ distributions are also shown, using ' $\mathrm{x}$ ' markers. (A free-stream Mach number of 0.5 is assumed 
here, otherwise the flow becomes supercritical for the highest value of $t / c$.) Increasing $t / c$ delays transition, however, the overall suction magnitude increases as a result of the associated rise in adverse pressure gradient over the rear of the aerofoil.

For the range of $C_{l}$ and $t / c$ relevant to the LFW aircraft, Fig. 4.9(a) shows that $t / c$ has a much greater effect on the overall suction requirement than $C_{l}$; the influence of the latter only becomes evident as $t / c$ is reduced below $15 \%$. The effect on $\widetilde{W}_{p}$ is slightly stronger, as detailed in Fig. 4.9(b). However, for the high thickness-to-chord ratios of interest in this study, the influence of $C_{l}$ on the overall suction requirement is likely to be negligible; consequently, variations in $C_{l}$ along the span of a properly designed LFW aircraft could possibly be neglected in initial calculations.

Although the boundary-layer calculation is incompressible, the Mach number has an effect via the aerofoil pressure distribution. The influence of free-stream Mach number and maximum section $t / c$ on $C_{Q}$ and $\widetilde{W}_{p}$ is detailed in Fig. 4.10. Each line of constant $t / c$ is plotted up to the Mach number at which the flow over the aerofoil first reaches the critical limit, in order to avoid shock waves (see Chap. 2). At the lower Mach numbers, the $C_{p}$ distribution for a given $C_{l}$ is unlikely to vary significantly; hence the initial weak dependence of $C_{Q}$ and $\widetilde{W}_{p}$ on $M_{\infty}$ for a fixed $t / c$ is relatively unsurprising. As $t / c$ and $M_{\infty}$ increase, the effect of $M_{\infty}$ becomes more noticeable, but not to the extent that it can be regarded as a significant influence. 


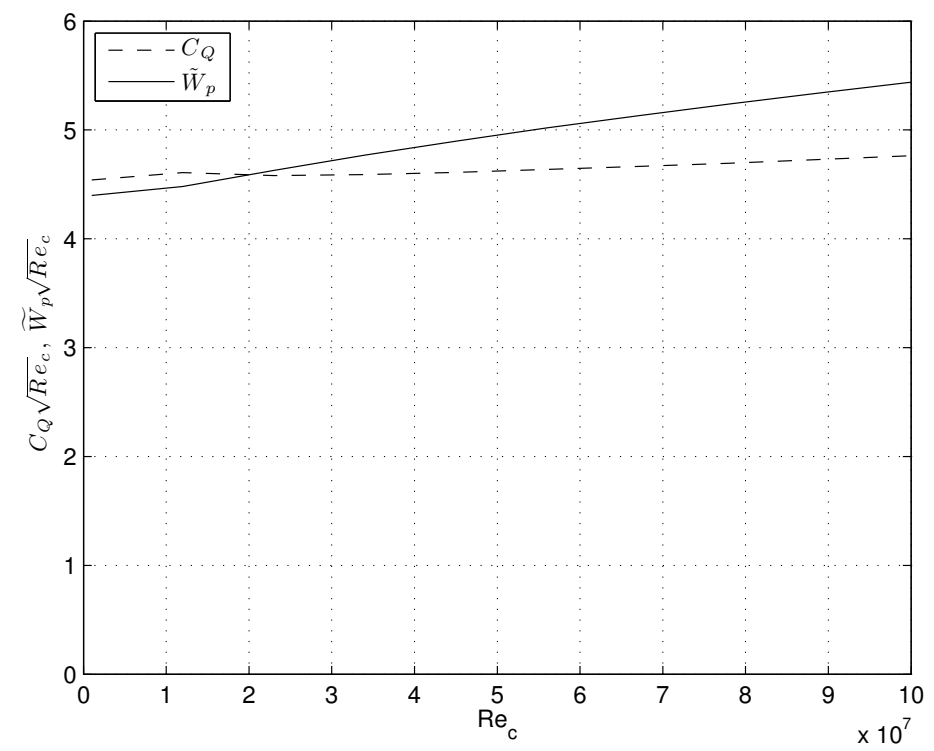

Figure 4.7: Variation of $C_{Q}$ and $\widetilde{W}_{p}$ with $R e_{c}$ : NACA0028, $C_{l}=0.15$ and $M_{\infty}=0.56$. Both variables are scaled by $\sqrt{R e_{c}}$, so that a constant level implies proportionality to $R e_{c}^{-1 / 2}$.

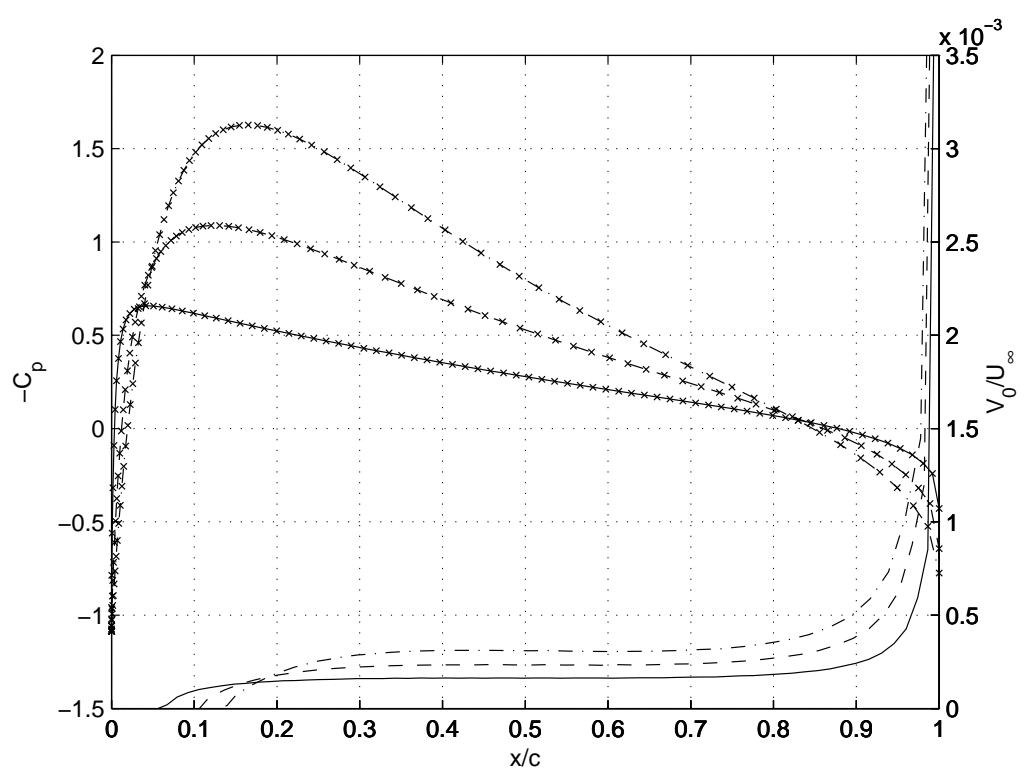

Figure 4.8: Dependence of distributed suction on section thickness for a range of symmetrical 4-digit NACA aerofoils at $C_{l}=0.15, M_{\infty}=0.5$ and $R e_{c}=40 \times 10^{6}$ : solid line $-10 \%$; dashed line $-20 \%$; and dashed-dotted line $-30 \%$; 'x' markers detail $C_{p}$. 


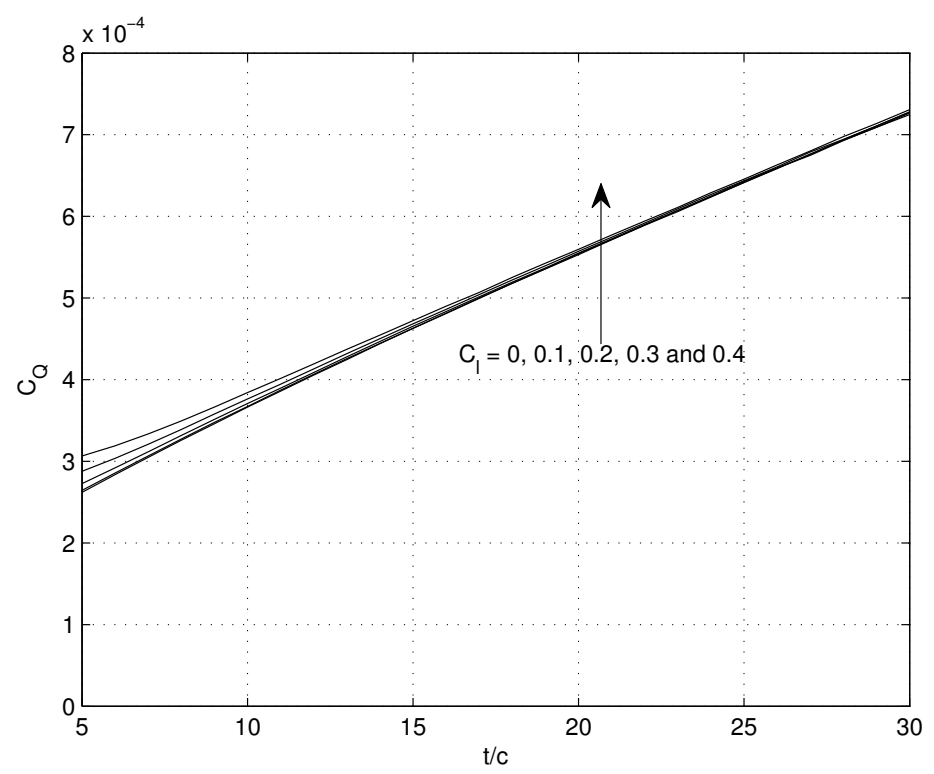

(a)

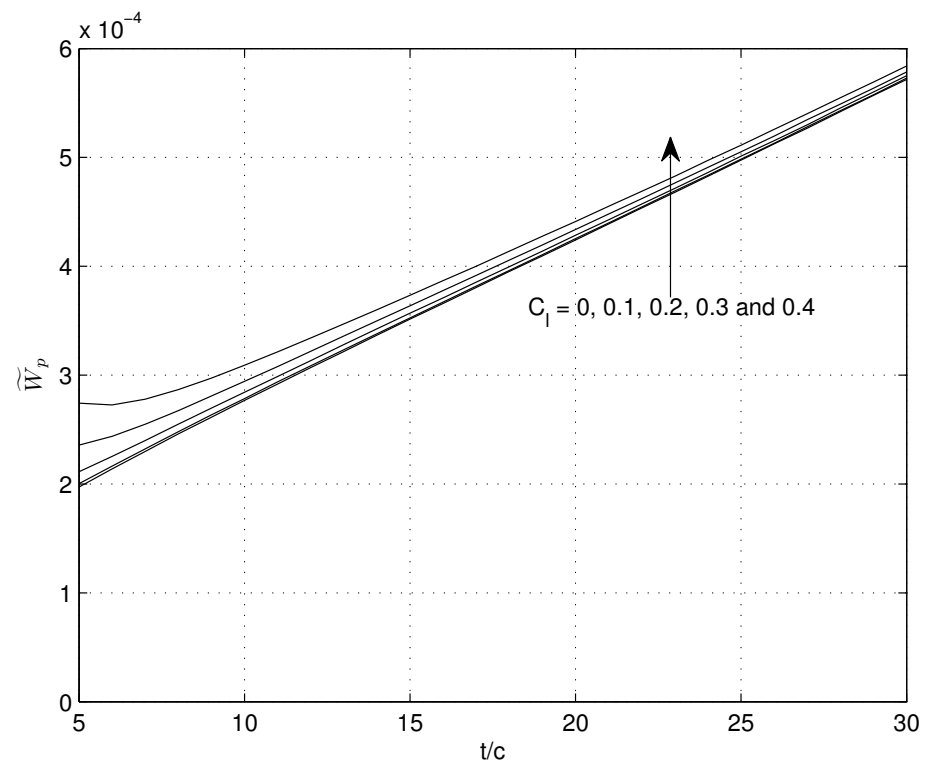

(b)

Figure 4.9: Variation of (a) $C_{Q}$ and (b) $\widetilde{W}_{p}$ with maximum thickness-to-chord ratio and section lift coefficient - symmetrical 4-digit NACA aerofoil series, $M_{\infty}=0.5$ and $R e_{c}=$ $40 \times 10^{6}$. 


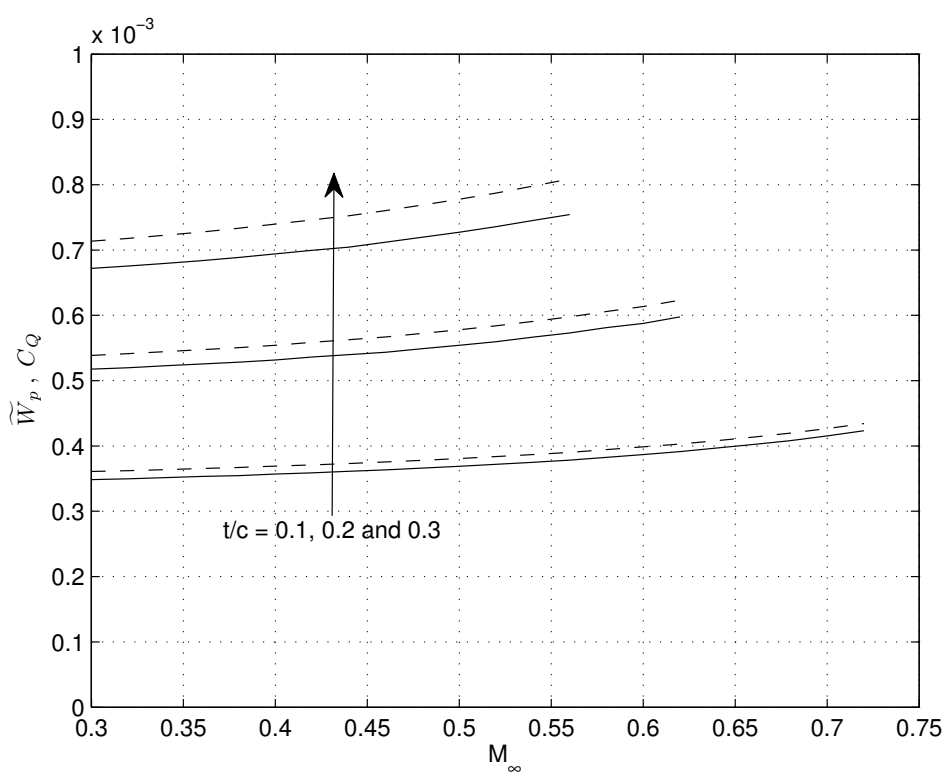

Figure 4.10: Variation of $C_{Q}$ (solid line) and $\widetilde{W}_{p}$ (dashed line) with free-stream Mach number and maximum thickness-to-chord ratio - symmetrical 4-digit NACA aerofoil series, $C_{l}=0.15$ and $R e_{c}=40 \times 10^{6}$.

\subsection{Suction Hardware Design and Power Consumption}

The power requirements associated with the inevitable loss in stagnation pressure through the boundary layer have been explored thus far. It now remains to specify a system to implement the LFC in practice. In this section a practical internal layout is proposed, consisting of spanwise chambers and chordwise ducts. The additional power demands associated with pressure drops across the porous skin, chambers and ducts are introduced in turn.

The typical LFW design parameter values and wing section geometry, specified in Sec. 4.3, are utilised. However, at this point, for clarity, considerations are restricted to the upper surface of the wing only. Spanwise variations in chord are also neglected.

\subsubsection{Suction Chamber Layout}

One possible internal pipework arrangement is a single collector duct receiving sucked air from all over the wing. However, pressure recovery over the rear of the aerofoil means that the driving pressure across the skin will increase significantly, making it difficult to attain the desired suction distribution with a constant skin resistance. Gregory (1961), and others 


\subsection{SUCTION HARDWARE DESIGN AND POWER CONSUMPTION}

such as Lachmann (1955), proposed that the ideal solution would be to have a number of pumps, or several stages in one pump, so that the applied pressure difference can be varied over the surface of the wing, thus keeping the flow rates across the skin to a minimum; or, alternatively, to grade the skin resistance. The latter approach, however, is a less efficient solution; although the correct flow rates are maintained, the pressure difference between the surface and pump entry will be greater than necessary in the high resistance regions.

A plan view of the proposed suction hardware configuration is illustrated in Fig. 4.11 the LFW aircraft would consist of several such arrangements along its span. The boundary-

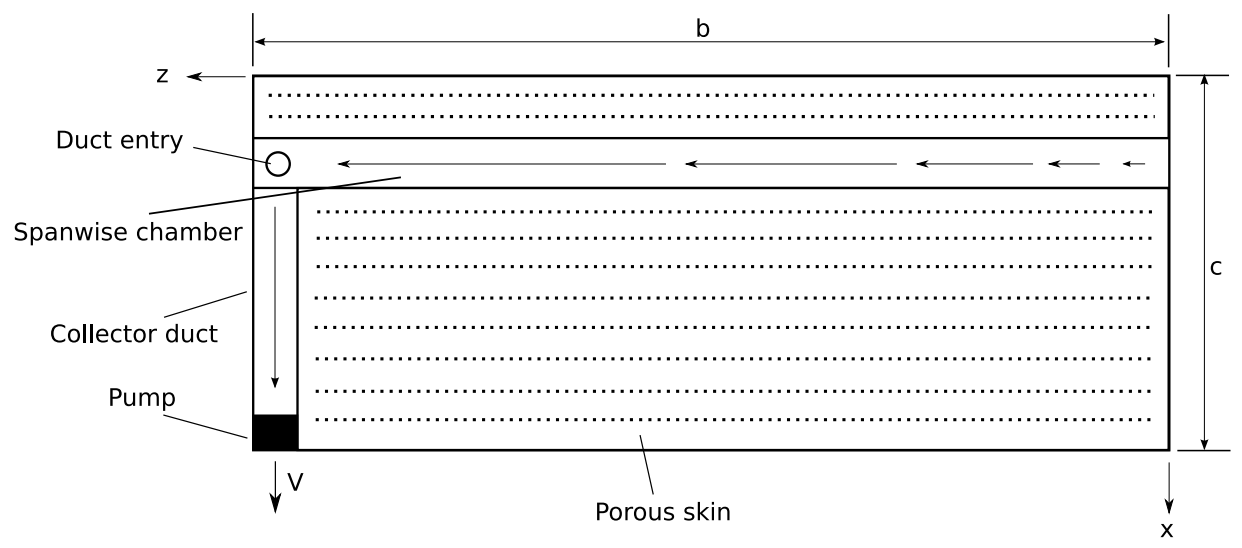

Figure 4.11: Suction hardware layout for an untapered wing of span $b$ and chord $c$.

layer fluid is sucked through the porous skin and then transported along spanwise chambers to a set of chordwise collector ducts; each duct feeds a pump, which ejects the sucked fluid into the free stream. Spanwise chambers, instead of chordwise, are adopted because they are structurally more efficient, allowing for integration with the main wing-box stiffeners (Pearce (1983)); furthermore, this arrangement minimises variations in the difference between the outer surface pressure and the (almost-constant) chamber pressure. Finally, given the space constraints imposed by a sharp-trailing-edge aerofoil, laminarisation is assumed possible up to $90 \%$ chord only.

\subsubsection{Skin Losses and Chamber Discretisation}

The chamber layout shown will provide a piecewise-constant subsurface pressure distribution. In this section, an algorithm is developed to specify this distribution, along with a skinresistance gradation, and then calculate the resulting 'real' suction flow. First, the analytical expression that relates the suction velocity across the porous skin to the applied pressure difference is presented. Then the algorithm is described, and the effect of parameter variations 


\section{CHAPTER 4. BOUNDARY-LAYER SUCTION SYSTEM DESIGN}

investigated. Finally, an approximate method for estimating the power associated with skin losses is given.

\subsubsection{Pressure Drop Across a Porous Skin}

The flow physics associated with sucking boundary-layer flow through a hole (or slot) has been well researched; here, in summarising the main features, reference is made to the works by Gregory (1961) and Thomas and Cornelius (1982). Essentially, there are two flow regimes: the external boundary-layer flow and the suction slot flow. For laminar flow applications, it is the flow immediately adjacent to the surface that is of importance in the first regime.

Gregory (1961) stated that the flow in the hole, and hence the pressure drop across the surface, is a function of three dimensionless groups: the hole Reynolds number, aspect ratio $\left(t_{s} / d\right)$ and Mach number. However, Thomas and Cornelius (1982), and other researchers such as Doerffer and Bohning (2000), have pointed out that the pressure drop also depends on the cross-flow velocity. Thomas and Cornelius (1982) thus added a fourth independent variable, characterising the boundary-layer flow immediately adjacent to the surface by a non-dimensional wall-velocity gradient.

Gregory (1961) commented that, for very low flow rates, the pressure drop is roughly proportional to the velocity through the hole - corresponding to the viscous type flow region over which D'Arcy's law holds; for larger flow rates, inertial effects become more important, with the pressure drop becoming proportional to the square of the hole velocity until, for large $R e_{h}, \frac{\Delta P_{s}}{\frac{1}{2} \rho_{w} V_{h}^{2}}=f n\left(R e_{h}\right) \sim$ const. For LFC applications, Poll et al. (1992) concluded that the flow through an individual hole is laminar, incompressible and pipe-flow-like, with the pressure drop having a quadratic dependence on the mass flow rate.

In spite of this body of work, no universal formula for the hole pressure drop has been agreed upon. Fortunately, for the present study, this obstacle can be circumvented as follows. On the basis of the existing literature, it may be asserted that a hole diameter which corresponds to a high-Reynolds-number and low-Mach-number flow regime can always be found. Furthermore, the external cross-flow at a given location is fixed, so now $\frac{\Delta P_{s}}{\frac{1}{2} \rho_{w} V_{h}^{2}} \sim$ const. This implies that the area-averaged suction velocity, in terms of the non-dimensional pressure difference across the skin $\Delta \widetilde{P}_{s}$, is given by

$$
\frac{V_{0}}{U_{\infty}}=K_{s} \sqrt{\frac{\rho_{\infty}}{\rho_{w}} \Delta \widetilde{P}_{s}}
$$

where $K_{s}$ depends on the chosen hole aspect-ratio and the skin porosity. The former is constrained by the original assertion, but it may be assumed that the latter remains under the control of the designer through the selection of a suitable value for $K_{s}$. 


\subsection{SUCTION HARDWARE DESIGN AND POWER CONSUMPTION}

In principle, this would allow for an optimised suction distribution to be realised with any chordwise $\Delta \widetilde{P}_{s}$ distribution. In reality, the associated continual porosity variation would be impractical. Instead, a single value of porosity, and hence $K_{s}$, associated with each chamber is suggested.

A final consideration is the flow temperature variation through an isolated hole in the porous surface. Doerffer and Bohning (2000) assume that the flow across a perforated plate is adiabatic. In contrast, Inger and Babinsky (2000) prefer to take it to be isothermal, although they also note that the temperature change calculated from an adiabatic assumption would be small (because the flow Mach numbers on entry and exit are likely to be similar). Neither approach appears to have been directly substantiated; given the low-Mach-number constraint here, both would give very similar results. However, the isothermal assumption prevents the calculation of the stagnation temperature at hole exit. Therefore, in this study, it is assumed that the flow is adiabatic. The stagnation temperature of the flow in the chamber is then equal to the static temperature on the external side of the skin.

\subsubsection{Chamber Specification Algorithm}

In the chordwise direction of a suction chamber, the pressure drop across the porous surface will change due to the surface pressure variation. As a result, the suction requirement to maintain laminar flow may no longer be satisfied (Lachmann (1955)). Therefore, it is necessary to determine a relationship between the suction required for laminarisation and that which can actually be achieved for a specified porous surface, as a function of the aerofoil's surface pressure distribution. First, the former is considered.

Eliminating $u_{e}^{\prime} / u_{e}$ from the boundary-layer integral equations, 4.1 and 4.2, and assuming suction maintains the boundary layer on the verge of transition such that $\delta_{2}^{\prime}=\delta_{3}^{\prime}=0$ :

$$
\left(2+H_{12}\right) \frac{\delta_{2}}{3 \delta_{3}}\left(C_{d}-\frac{V_{0}}{u_{e}}\right)=C_{f}-\frac{V_{0}}{u_{e}}
$$

Under these assumptions, the shape factor $H_{32}$ is constant, so both $C_{f}$ and $C_{d}$ vary only with the external (potential) flow velocity ratio $u_{e} / U_{\infty}$, as $\left(u_{e} / U_{\infty}\right)^{-1}$. For incompressible flow the velocity ratio is related to $C_{p}$ by:

$$
\frac{u_{e}}{U_{\infty}}=\sqrt{1-C_{p}}
$$


Hence the scaling for the required $V_{0} / u_{e}$ is:

$$
\left(\frac{V_{0}}{u_{e}}\right)_{\text {required }} \sim \frac{1}{\sqrt{1-C_{p}}} .
$$

For the practical, chamber-based, implementation here, the skin pressure drop is:

$$
\Delta \widetilde{P}_{s}=C_{p}+\widetilde{P}_{\infty}-\widetilde{P}_{c}
$$

Combining this with Eq. 4.8, the dependence of $V_{0}$ on $C_{p}$ is:

$$
\left(\frac{V_{0}}{u_{e}}\right)_{\text {actual }} \sim \sqrt{\frac{k+C_{p}}{1-C_{p}}},
$$

where $k=\widetilde{P}_{\infty}-\widetilde{P}_{c}$ is a constant.

Combining Eqs. 4.11 and 4.13:

$$
\frac{\left(\frac{V_{0}}{U_{\infty}}\right)_{\text {actual }}}{\left(\frac{V_{0}}{U_{\infty}}\right)_{\text {required }}} \sim \sqrt{k-\left(-C_{p}\right)} .
$$

Equation 4.14 reveals that, as $-C_{p}$ drops downstream of the suction peak, $\left(V_{0} / U_{\infty}\right)_{\text {actual }}$ increases at a faster rate than $\left(V_{0} / U_{\infty}\right)_{\text {required }}$; so choosing $K_{s}$ to meet the suction requirement at the upstream edge of the chamber should ensure successful laminarisation across its entire width. On the other hand, laminarisation issues may arise with the increasing $-C_{p}$ upstream of the suction peak. The former, more usual, case is considered first.

Figure 4.12 shows a schematic diagram of the internal and external pressures across a single chamber. The minimum and maximum pressure differences, $\Delta \widetilde{P}_{s, \text { min }}$ and $\Delta \widetilde{P}_{s, \max }$, are design parameters which implicitly define the chamber width $w_{c}$. The required skin constant $K_{s}$ is evaluated from Eq. 4.8, using $\Delta \widetilde{P}_{s}\left(=\Delta \widetilde{P}_{s, \min }\right)$ and the optimal suction velocity $\left(V_{0} / U_{\infty}\right)_{\text {opt }}$ at the upstream end of the chamber. The calculation proceeds downstream, calculating $\left(V_{0} / U_{\infty}\right)_{\text {actual }}$ - continuously checking the laminar flow condition (since Eq. 4.14 is only an approximate guide) - until $\Delta \widetilde{P}_{s}>\Delta \widetilde{P}_{s, \text { max }}$. The optimal suction velocity at the start of the next chamber is then calculated on the basis of the current boundary-layer state.

For large $\Delta \widetilde{P}_{s, \max }$, the boundary layer may be stabilised to such an extent that the required $\left(V_{0} / U_{\infty}\right)_{\text {opt }}$ at the start of the next chamber is negligible and, hence, the initial estimate of $K_{s}$ too small to maintain laminar flow over that chamber. If this issue arises, $K_{s}$ is increased iteratively until transition is avoided. 


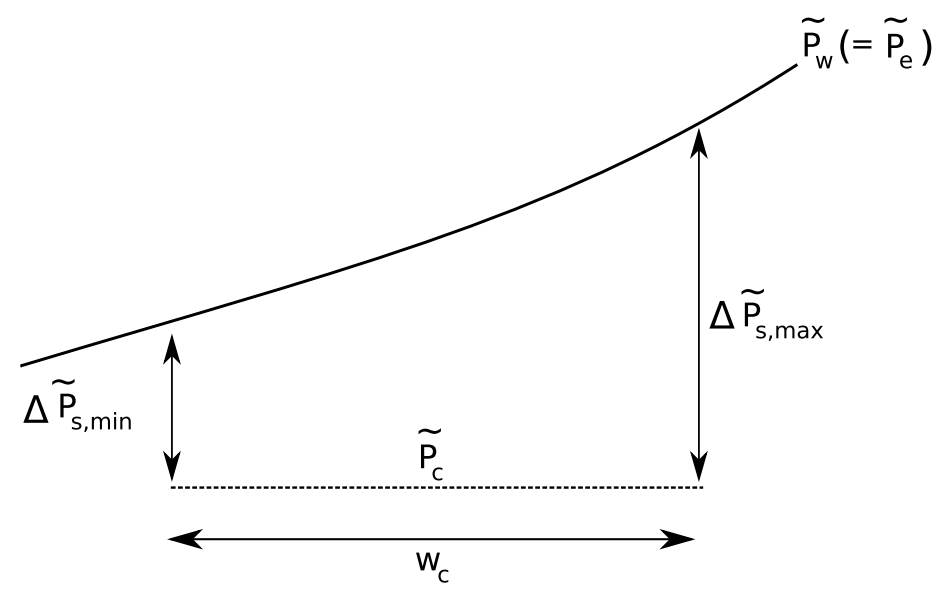

Figure 4.12: Schematic diagram of surface pressure variation over a single chamber width (downstream of the suction peak).

Upstream of the suction peak, the locations of $\Delta \widetilde{P}_{s, \min }$ and $\Delta \widetilde{P}_{s, \text { max }}$ switch, as does the prediction of Eq. 4.14 for the most critical point. The skin constant is thus still set with $\Delta \widetilde{P}_{s, \min }$, but using an optimum suction velocity calculated at the downstream end of the chamber. The actual suction distribution is then evaluated starting from the upstream end, as usual.

In summary, a chamber's pressure is set by specifying $\Delta \widetilde{P}_{s, \min }: \widetilde{P}_{c}=\widetilde{P}_{e}-\Delta \widetilde{P}_{s, \min }$; and its chordwise extent is determined by $\Delta \widetilde{P}_{s, \max }$. Both of these parameters are constrained by the incompressible flow assumption implicit in the definition of the skin constant $K_{s}$. (The practical benefit of this constraint is an avoidance of possible choking problems that might otherwise arise.)

\subsubsection{Chamber Constraint Trade-Off Study}

To understand the influence of $\Delta \widetilde{P}_{s, \min }$ and $\Delta \widetilde{P}_{s, \max }$ on the suction power requirement, the following parameters are introduced: $\Delta \widetilde{P}_{s, a v}=\frac{1}{2}\left(\Delta \widetilde{P}_{s, \min }+\Delta \widetilde{P}_{s, \max }\right)$ and $\tilde{r}_{c}=\frac{\Delta \widetilde{P}_{s, \max }}{\Delta \widetilde{P}_{s, \min }}$. The first term characterises the resistance of the porous surface, whilst the latter influences the sensitivity of the suction distribution over a chamber. The maximum value of each parameter is restricted by the incompressible flow assumption across the skin; a suitable range is $0.05 \leq \Delta \widetilde{P}_{s, a v} \leq 0.27$ and $2 \leq \widetilde{r}_{c} \leq 4$. Figure 4.13 details the chordwise variation of $\Delta \widetilde{P}_{s}$ corresponding to the upper limits specified, which give the greatest pressure difference; the critical pressure differential, corresponding to a hole Mach number of $0.4\left(P_{c} / P_{0, e}=0.8703\right)$, is superimposed.

Before exploring the suction requirements, consider Fig. 4.14, which shows the number 


\section{CHAPTER 4. BOUNDARY-LAYER SUCTION SYSTEM DESIGN}

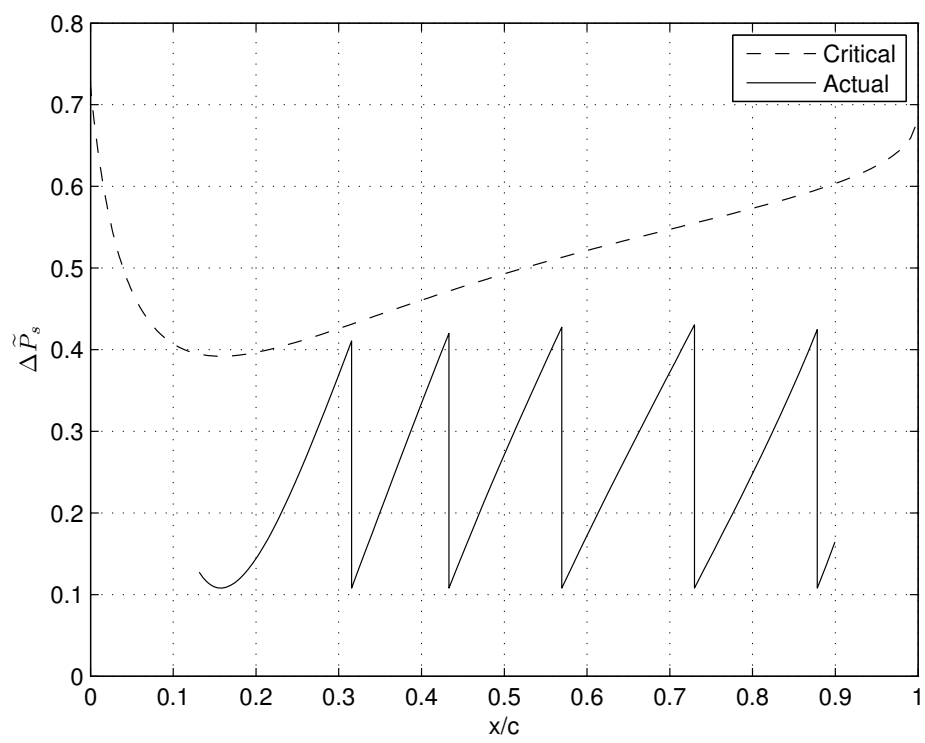

Figure 4.13: Chordwise distribution of differential pressure across the porous skin with the critical pressure superimposed.

of chambers as a function of $\Delta \widetilde{P}_{s, a v}$ and $\tilde{r}_{c}$. Here we see that, for a given $\tilde{r}_{c}$, the number reduces significantly initially, and then more gradually, with increasing $\Delta \widetilde{P}_{s, a v}$. This is due to the growing difference between $\Delta \widetilde{P}_{s, \min }$ and $\Delta \widetilde{P}_{s, \max }$. The rate of reduction decreases for higher values of $\tilde{r}_{c}$; however, increasing $\tilde{r}_{c}$ for a given $\Delta \widetilde{P}_{s, a v}$ results in fewer chambers.

The associated suction coefficient is plotted in Fig. 4.15. $C_{Q}$ is remarkably insensitive to $\Delta \widetilde{P}_{s, a v}$ and $\tilde{r}_{c}$. The former dependence is unsurprising, given the varying skin resistance used to meet suction targets. To understand the latter, consider Fig. 4.16, which details the variation of the chordwise distribution of suction with $\Delta \widetilde{P}_{s, a v}$ and $\tilde{r}_{c}$ for the combination of extreme values. Increasing $\tilde{r}_{c}$ increases the fluctuation of the suction distribution, but the average level of suction is insignificantly affected; oversuction towards the end of a chamber is almost exactly compensated by a reduced requirement at the start of the next. Figure 4.16 also confirms that increasing $\Delta \widetilde{P}_{s, a v}$ does not significantly affect the fluctuation, or overall magnitude, of the suction.

The corresponding $\widetilde{W}_{p}$ variations are plotted in Fig. 4.17, showing that this parameter has a greater dependence on $\Delta \widetilde{P}_{s, a v}$ compared to $\tilde{r}_{c}$. This is unsurprising given the suction velocity behaviour, which implies that the surface resistance correlates almost solely with $\Delta \widetilde{P}_{s, a v}$. The small increase in power with $\tilde{r}_{c}$ is attributed to the redistribution of suction, nearer the suction peak, where the stagnation pressure loss through the boundary layer is 


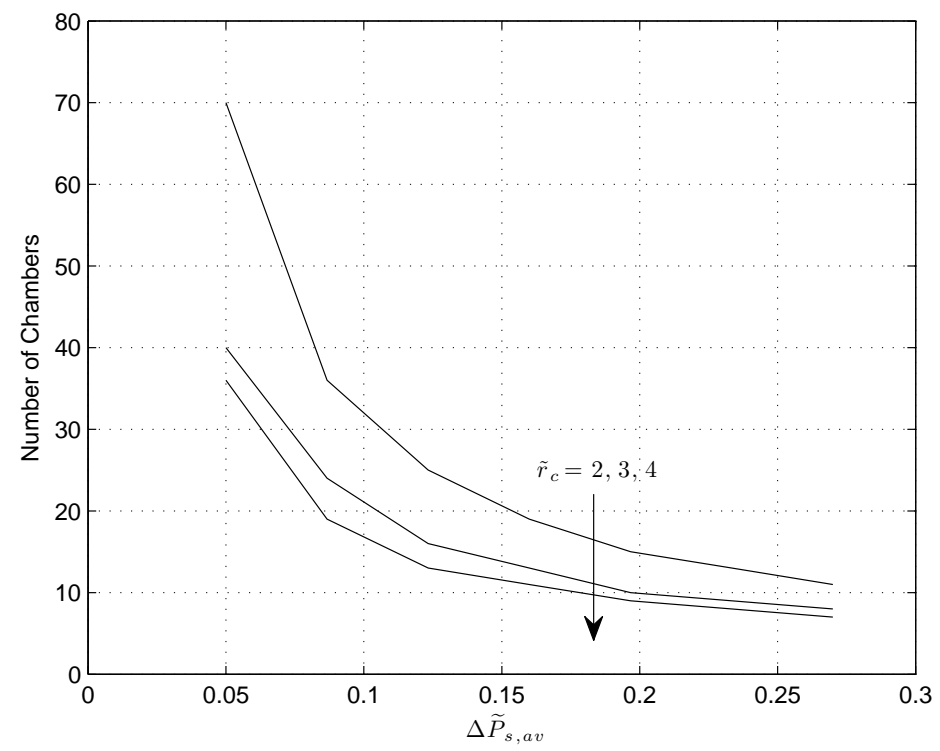

Figure 4.14: Variation of the total number of chambers with $\Delta \widetilde{P}_{s, a v}$ and $\tilde{r}_{c}$.

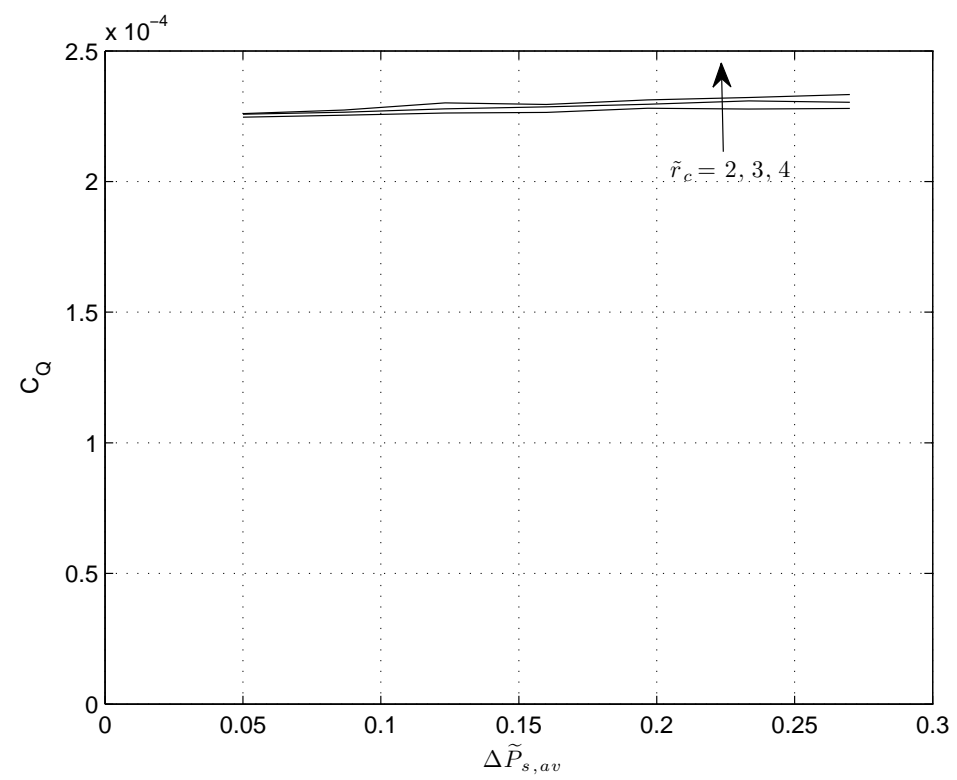

Figure 4.15: Dependence of $C_{Q}$ on $\Delta \widetilde{P}_{s, a v}$ and $\tilde{r}_{c}$. The optimal value calculated by the algorithm of Sec. 4.1.2 is $C_{Q}=2.24 \times 10^{-4}$.

greatest. This effect is most prominent at higher values of $\Delta \widetilde{P}_{s, a v}$, as can be deduced from the chordwise distribution of suction detailed in Fig. 4.16. 


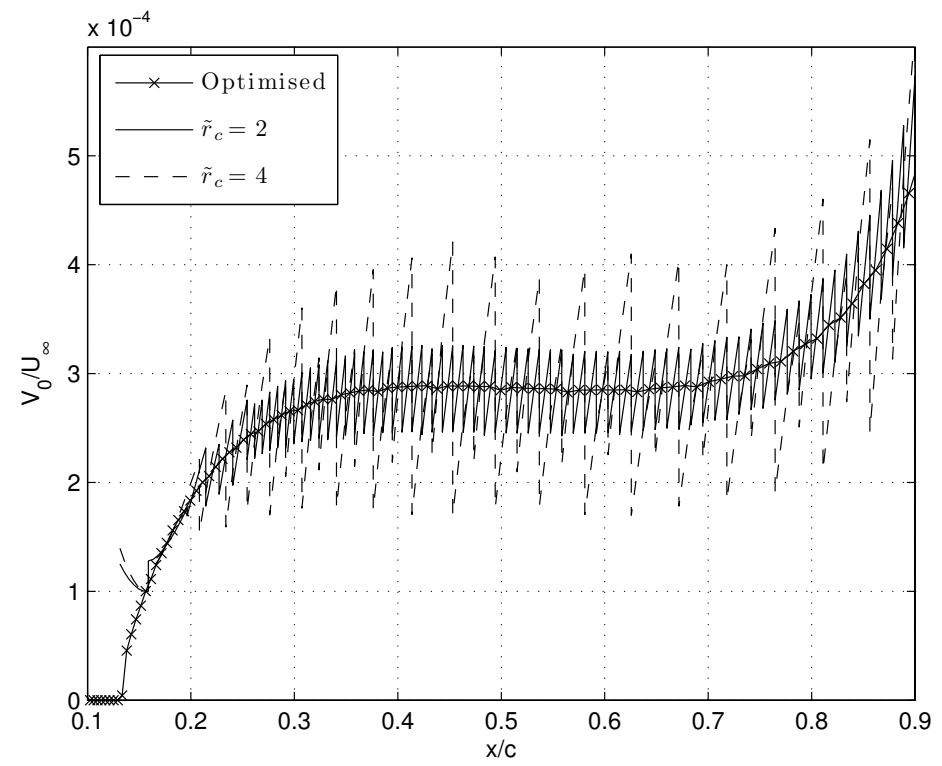

(a) $\Delta \widetilde{P}_{s, a v}=0.05$ (for $\tilde{r}_{c}=2$ and $4, C_{Q}=2.25 \times 10^{-4}$ and $2.26 \times 10^{-4}$, respectively).

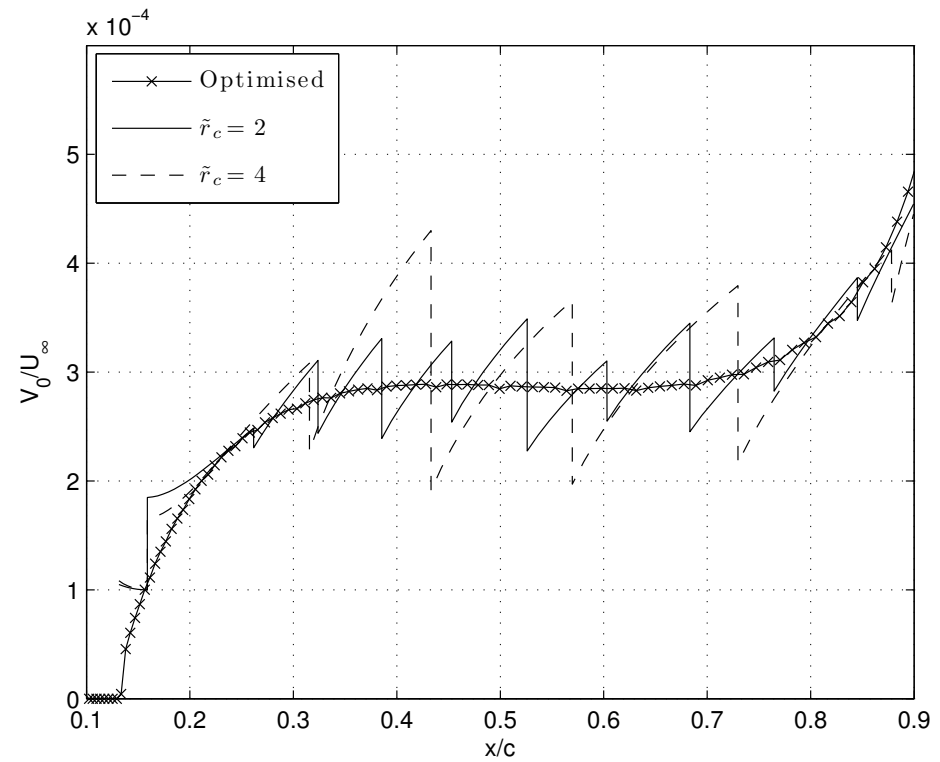

(b) $\Delta \widetilde{P}_{s, a v}=0.27$ (for $\tilde{r}_{c}=2$ and $4, C_{Q}=2.28 \times 10^{-4}$ and $2.33 \times 10^{-4}$, respectively).

Figure 4.16: Variation of chordwise distribution of suction with $\tilde{r}_{c}$ and $\Delta \widetilde{P}_{s, a v}$. 


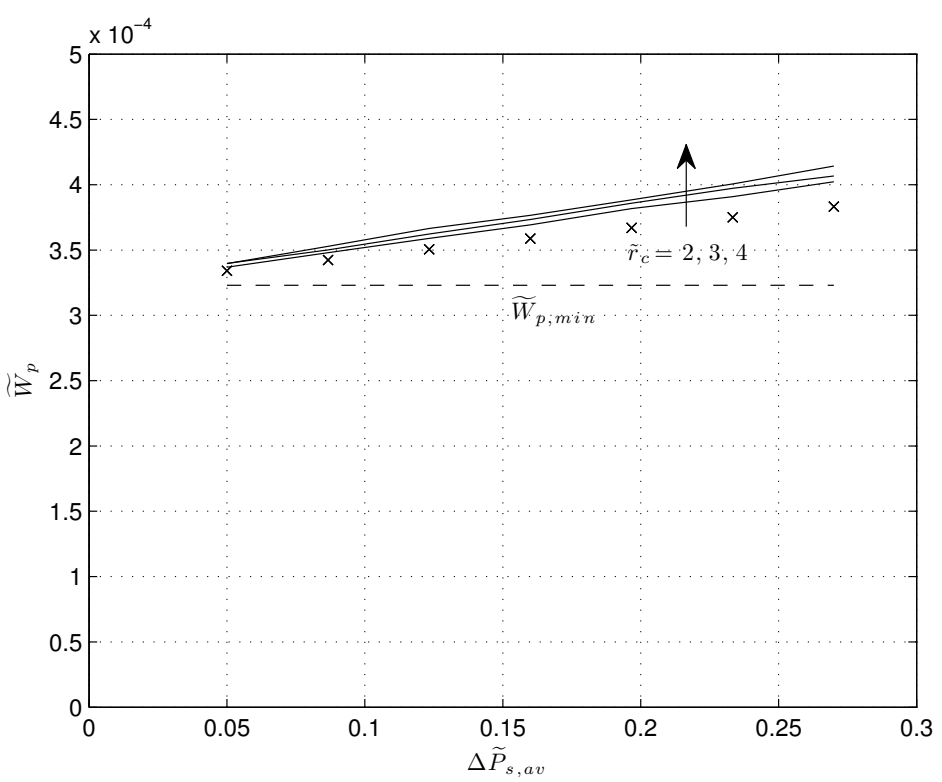

Figure 4.17: Variation of actual (solid lines) and approximate ('x' markers) $\widetilde{W}_{p}$ with $\Delta \widetilde{P}_{s, a v}$ and $\tilde{r}_{c}$.

The almost-linear dependence of $\widetilde{W}_{p}$ on $\Delta \widetilde{P}_{s, a v}$ suggests that it may be possible to find a simple approximate relationship between the two. First, note that due to the Mach-number constraint, the hole flow will be not far from incompressible. If, additionally, variations in the pressure difference are neglected, the power consumption associated with the skin is

$$
\widetilde{W}_{p, s} \approx C_{Q} \Delta \widetilde{P}_{s, a v}
$$

Finally, the minimal dependence of $C_{Q}$ on the chamber design parameters means that it can be approximated by its optimal value. Adding the minimum power requirement associated with the optimised suction distribution to Eq. 4.15 gives an approximate estimate of the total power, which is also plotted in Fig. 4.17. There is excellent agreement between the two approaches; however, the incompressible flow assumption does begin to underestimate the power requirement somewhat at higher pressure differences, due to the differences between optimal and actual $C_{Q}$ s, and the neglect of compressibility (accounted for in Eq. 4.6).

Given the dependencies of the total number of chambers and power requirement, a reasonable choice of parameters might be $\Delta \widetilde{P}_{s, a v}=0.15$ and $\tilde{r}_{c}=3$. The number of chambers is then $13, C_{Q}$ is $2.28 \times 10^{-4}$ and $\widetilde{W}_{p}$ is $3.70 \times 10^{-4}$. A breakdown of $\widetilde{W}_{p}$ into the losses across the boundary layer and the skin is $3.32 \times 10^{-4}$ and $0.38 \times 10^{-4}$, respectively; in comparison, the optimised suction distribution, with stagnation pressure loss through the boundary layer 


\section{CHAPTER 4. BOUNDARY-LAYER SUCTION SYSTEM DESIGN}

alone, has $\widetilde{W}_{p}=3.23 \times 10^{-4}$. Therefore the redistribution of suction has had little adverse effect on the power requirement, and the increase due to the resistance of the porous skin is small, contributing only $10 \%$ to the total power.

\subsubsection{Spanwise Chamber Losses}

With the chamber widths and baseline pressures now fixed, it is necessary to introduce the influence of spanwise chamber losses. By discretising the chamber in the spanwise direction, the pressure drop is calculated numerically. The sucked mass flow into the first element is known from the chamber pressure set to pass the flow across the porous skin. However, the chamber pressure then drops in the spanwise direction (due to inertial and wall-friction effects), the differential pressure across the skin increases and, consequently, so does the suction mass flow into the chamber. For each spanwise element, the pressure drop and suction rate are calculated iteratively. The converged chamber pressure is then used to begin the iteration over the next element. The laminar flow condition is also checked at each stage.

In analysing the flow along a chamber, it is assumed that the chamber velocities are low enough to warrant the assumption of incompressible flow. By application of the 1D momentum and mass continuity equations, and accounting for wall friction, the static pressure gradient over an element of length $\delta z$, width $w_{c}$ and height $h$, as illustrated in Fig. 4.18, is:

$$
\frac{d P}{d z}=-\frac{2 \dot{m}^{2}}{\rho_{c} h^{2}}\left(\frac{\dot{m}_{s}}{\dot{m}}+\frac{f}{D_{e q}}\right)
$$

The friction factor $f$ is a function of $R e_{D_{e q}}$ and $D_{e q}$ - the latter influence is due to roughness effects. The equivalent diameter $D_{e q}=2 h \frac{w_{c} / h}{1+w_{c} / h}$ must be used for rectangular conduits; and subsequently, $R e_{D_{e q}}=\frac{2 \dot{m}}{\mu} \frac{w_{c} / h}{1+w_{c} / h}$. The friction factor is calculated using the standard relation for laminar flow, whilst Churchill's correlation is used for turbulent flow; furthermore, the chambers are assumed to have the same roughness as drawn tubing (Menon (2004)).

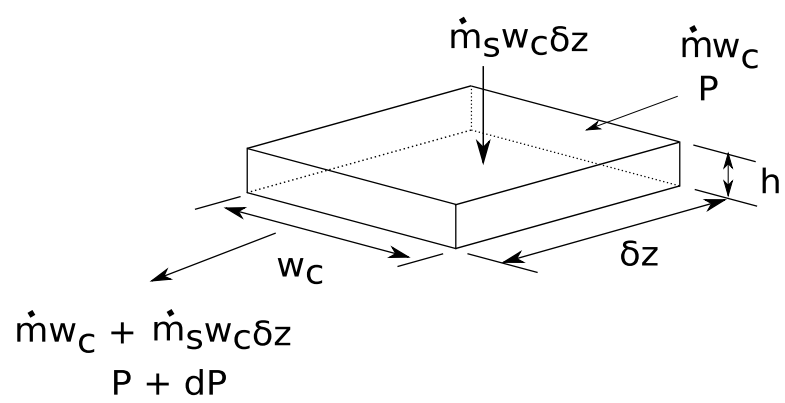

Figure 4.18: Notation for chamber flow. 


\subsection{SUCTION HARDWARE DESIGN AND POWER CONSUMPTION}

The relevant geometrical parameters affecting the pressure drop over a span $b$ are $b / h$ and $w_{c} / h$ (through $D_{e q}$ ). To understand the influence of these parameters, the pressure loss along a single spanwise chamber is investigated, with external flow properties equivalent to those at an altitude of $10900 \mathrm{~m}$ and $M_{\infty}=0.56$, and having: a constant external chordwise suction distribution of $V_{0} / U_{\infty}=3.29 \times 10^{-4}$; a skin porosity $K_{s}=11.0 \times 10^{-4}$; and typical surface and chamber pressures of $\widetilde{P}_{e}=4.58$ and $\widetilde{P}_{c}=4.49$, respectively.

Figure 4.19 details the variation of $\Delta \widetilde{P}_{c}$ with $b / h$ and $w_{c} / h$, for a constant chamber depth $h$ of $10 \mathrm{~cm}$. As expected, there is a strong dependence on $b / h$. Initially, $\Delta \widetilde{P}_{c}$ decreases with $w_{c} / h$, and then becomes independent of this parameter for higher values. Plots for other values of $h$ are very similar, but not identical, due to Reynolds number dependence.

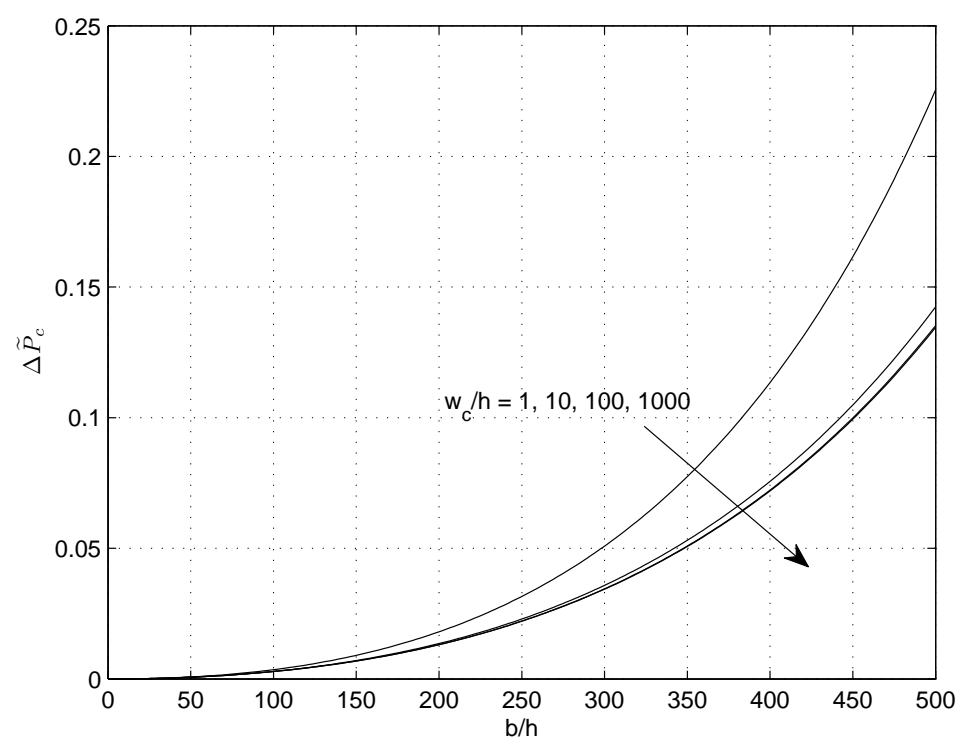

Figure 4.19: Spanwise-chamber pressure drop dependence on $b / h$ and $w_{c} / h$ with $h=10 \mathrm{~cm}$.

In order to interpret these results, it is helpful to assume that the pressure drop is small enough to warrant the approximation $\dot{m}_{s} \approx$ constant; and that the friction factor is constant along the length of a chamber. Then, on integration of Eq. 4.16, the non-dimensional chamber pressure drop becomes:

$$
\Delta \widetilde{P}_{c}=-\frac{2 \dot{m}_{s}^{2}}{\rho_{c} \rho_{\infty} U_{\infty}^{2}}\left(\frac{b}{h}\right)^{2}\left[1+\frac{f}{3}\left(\frac{b}{h}\right)\left(\frac{1+w_{c} / h}{w_{c} / h}\right)\right]
$$

Equation 4.17 suggests that the inertial term's contribution to the pressure drop is approximately quadratic in $b / h$, and the friction term's is cubic. The explicit dependence on $w_{c} / h$ is 


\section{CHAPTER 4. BOUNDARY-LAYER SUCTION SYSTEM DESIGN}

restricted to the friction term, and disappears for wide, shallow chambers. These observations are consistent with the results of Fig. 4.19.

From the design viewpoint, Eq. 4.17 shows that the spanwise extent of chambers must be limited to avoid excessive losses. If the friction contribution is not to exceed the inertial, and we take $f \simeq 0.01$, then we require $b / h \lesssim 300$ (for shallow chambers). At a typical value of $\dot{m}_{s}^{2}, \dot{m}_{s}^{2} / \rho_{w} \rho_{\infty} U_{\infty}^{2} \simeq 10^{-7}$, giving $\Delta \widetilde{P}_{c} \simeq 0.036$ at the upper limit. Thus, for example, $10 \mathrm{~cm}$ deep chambers could run for $30 \mathrm{~m}$ with negligible pressure drop, suggesting that the power losses from this source should be avoidable in a practical design.

\subsubsection{Collector Duct Trade-Off Study}

The power calculations in Sec. 4.4.2 correspond to a system architecture having one collector duct and pump per chamber. This, however, is likely to become impractical for a large number of chambers. An alternative arrangement where the flow from multiple chambers is collected by a single collector duct is detailed schematically in Fig. 4.20. As the required static pressures of adjacent chambers $\left(P_{c, 1}, P_{c, 2}, P_{c, 3}\right.$ etc) differ, flow rate control devices (e.g. orifice plates) are then needed. The collector duct stagnation pressure $P_{0, d}$ is set by the lowest static pressure among the chambers which feed it. (It is assumed here that the collector ducts are large enough for skin friction losses to be negligible.)

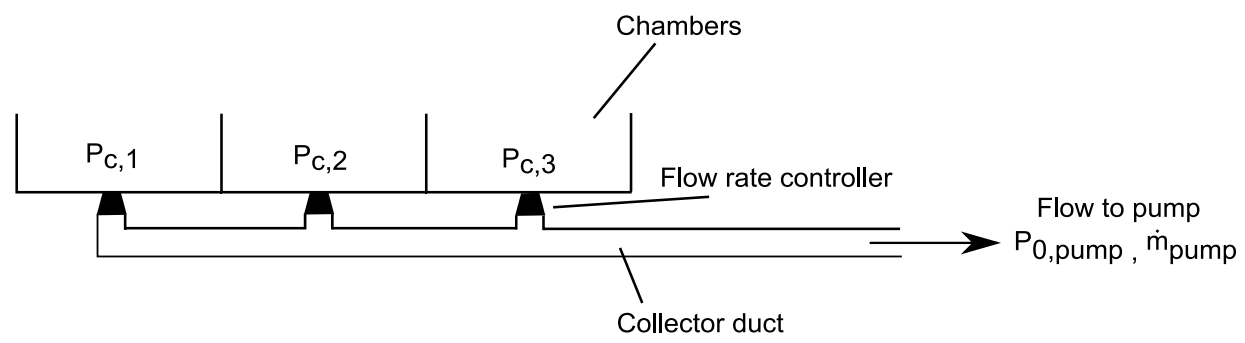

Figure 4.20: Schematic diagram of an alternative chamber-collector-duct arrangement.

It is necessary to understand the trade-off between the power requirement and the number of chambers and collector ducts. The final number of ducts (and pumps) must be based on a consideration of geometric constraints and power requirements. More ducts imply increased complexity, but lower average pressure loss from chamber to pump.

The pressure losses associated with throttling the flows from the chambers can be minimised by having an approximately equal number of chambers per collector. If the total chamber number is not an integer multiple of the collector number, the first residual chamber is added to the last collector arrangement, and the remainder allocated to successive arrangements starting from the first. Figure 4.21 provides an example illustration of the 
pressure distribution corresponding to 13 chambers and four collectors - each collector has a minimum of three chambers, with the last having four.

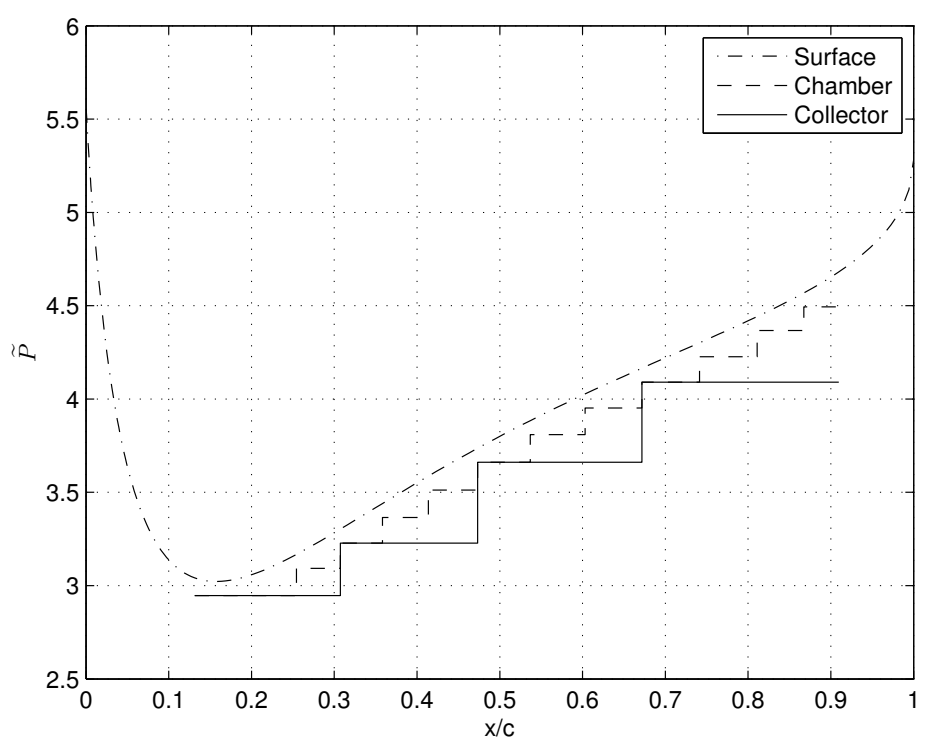

Figure 4.21: Comparison of surface, chamber and collector static pressures $-\Delta \widetilde{P}_{s, a v}=0.15$ and $\tilde{r}_{c}=3$.

The variation of $\widetilde{W}_{p}$ with the total number of collector ducts, for different values of $\tilde{r}_{c}$, is detailed in Fig. 4.22. Consider the case where $\tilde{r}_{c}=2$ (which gives 19 chambers) first: one collector duct, having a pressure offset from the suction peak pressure of $\Delta \widetilde{P}_{s, a v}(=0.15)$, gives the greatest power requirement, but $\widetilde{W}_{p}$ starts to asymptote very quickly; by five collectors the power penalty is only $10 \%$. As $\tilde{r}_{c}$ is increased, the number of chambers, and hence the maximum number of collector ducts decreases; interestingly, this insignificantly affects the power variation and its magnitude. Therefore, for a given number of collector ducts and average skin pressure drop, there are no direct benefits to be had from increasing the number of chambers and trying to maintain a near-optimal suction distribution. (There may, nonetheless, be indirect benefits, specifically if the chamber sidewalls also have a role as structural stiffeners.) This observation, should, in fact, be no great surprise: the overall average pressure drop between the suction surface and the pumps is now set by the number of collector ducts, and the only potential benefit in having more chambers is to ensure a suction distribution closer to optimal. However, as we have seen, departures from the optimum have very little effect on overall suction volumes, and hence also on power consumption. 


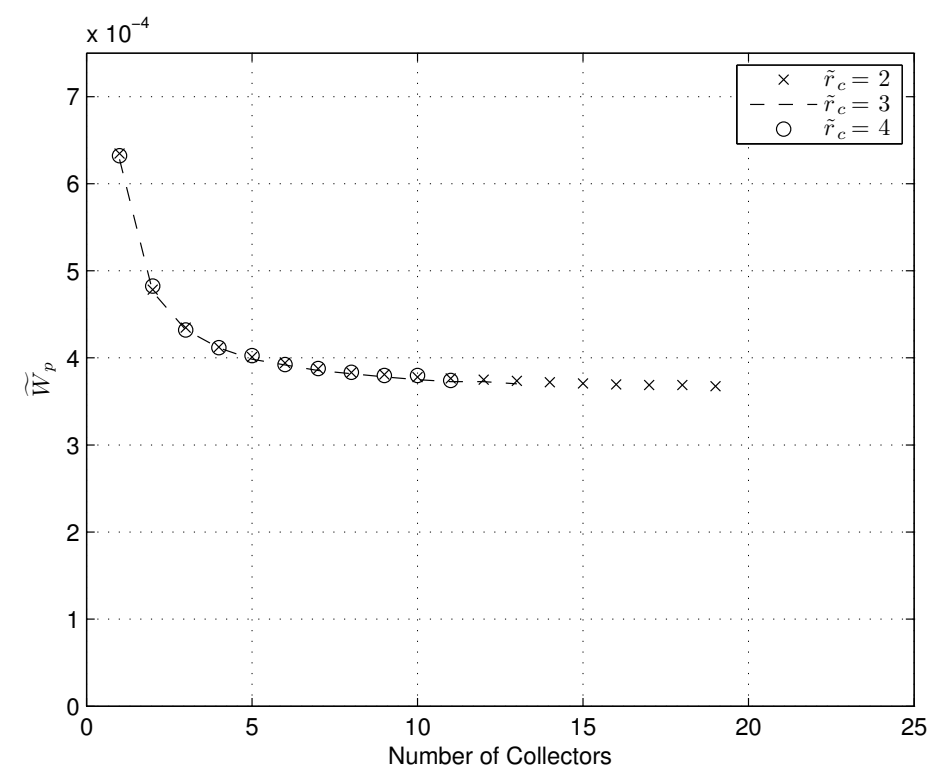

Figure 4.22: Variation of $\widetilde{W}_{p}$ with the number of collectors and $\tilde{r}_{c}$, with $\Delta \widetilde{P}_{s, a v}=0.15$.

\subsection{Summary}

The Eppler and Somers (1980) semi-empirical boundary-layer stability algorithm has been implemented to determine the optimal distribution of suction required to maintain laminar flow over a desired region, for a specified aerofoil geometry, surface pressure distribution and chord Reynolds number. To facilitate the design of a practical suction system, an automated algorithm has been developed. It requires, only, the specification of the porous skin's surface resistance and the sensitivity of the suction distribution (represented by an average pressure drop across the skin, $\Delta \widetilde{P}_{s, a v}$, and the maximum-to-minimum pressure difference ratio, $\tilde{r}_{c}$, across a chamber), to define a set of subsurface chamber widths and pressures. The method assumes that, for a given chamber pressure distribution, the corresponding real suction distribution and power calculation can be found without needing to calculate suction hole velocities explicitly; however, the pressure difference across the skin should be such that flow remains subsonic (and incompressible). The spanwise chamber pressure drop required to overcome frictional and inertial effects has been investigated. Finally, the effects of collector ducts (introduced to reduce system architecture complexity) on the power requirement have been explored.

The study identified a minimum power requirement independent of the suction system design; this is associated with the boundary-layer stagnation-pressure loss of the flow in an 
optimal suction distribution (one which maintains a neutrally stable laminar boundary-layer). Deviations from the optimal suction distribution, due to a practical porous-skin and suctionchamber architecture, have very little effect on the overall suction coefficient, but additional power is required, due to the skin resistance. To a good approximation, this power penalty is given by the product of the optimal suction flow-rate coefficient and the average skin pressure drop. In the spanwise direction, the effect of the chamber pressure drop due to frictional and inertial effects is two-fold: firstly, it gives rise to excess suction, and secondly, it combines with the excess suction to increase the pressure losses. However, in practise, through suitable choice of spanwise-length-to-chamber-depth, it should be possible to make this contribution to the overall power requirement negligible. Finally, if there are fewer collector ducts (and hence pumps) than chambers, it is the average pressure drop from the aerofoil surface to the collector ducts, rather than to the chambers, that determines the power penalty.

With regard to the conceptual design of a LFW, a suction system consuming little more power than the optimum value is a practical possibility. However, a representative aerofoil pressure distribution and associated suction profile are necessary to obtain an accurate estimate of this optimum. Once these are available, the system architecture may be specified by the following algorithm:

(1) set the average skin pressure drop, $\Delta \widetilde{P}_{s, a v}$;

(2) decide the number of suction pumps;

(3) choose the pressure ratio parameter $\tilde{r}_{c}$ so that the number of chambers is equal to the number of pumps;

(4) check suction hole Mach numbers and, if too high, iterate from (2);

(5) estimate skin resistance power penalty and, if necessary (either the penalty or the number of pumps is too high) iterate from (1);

(6) set the chamber depth so that spanwise pressure losses are negligible (c.f. equation 4.17).

On the basis of the typical LFW design parameters used in this study, such an approach can be expected to produce a design consuming at most $20 \%$, and more likely around $10 \%$, excess power over the optimum value. 


\section{CHAPTER 5}

\section{LFW Aircraft And Mission SPECIFICATION}

This chapter begins, in Sec. 5.1, by developing a high-level design approach. In particular, the approach starts from the conditions that the aircraft will be a LFW operating at near-optimum aerodynamic efficiency, and that its suction requirements must be essentially determinable from two-dimensional boundary-layer calculations. A discussion of the detailed constraints which result from these conditions follows, and it is shown how they may be combined to yield a combined aircraft/mission specification, given values for three principal design variables: span, thickness-to-chord ratio and unit Reynolds number. In Sec. 5.2 the methodology and analytical models used for the calculations are described. Then, in Sec. 5.3, a parametric study is undertaken to investigate the effects of the principal design variables on the aircraft/mission specification. Finally, the choice of design variables for a detailed study, which will follow in Chap. 6, is discussed in Sec. 5.4.

\section{$5.1 \quad$ Design Approach}

The mission specification that will be defined here consists of the cruise speed and altitude (the range of the LFW will be an output of the subsequent conceptual design work). This immediately sets one parameter of fundamental importance for all aircraft - the Mach number - and one that Lachmann (1955) highlights as crucial to LFC applications: the unit Reynolds number, $U_{\infty} / \nu$. Figure 5.1 shows how Mach number and unit Reynolds number vary with altitude and speed, according to the American Standard Atmosphere (1976). Selfevidently, the unit Reynolds number at a given altitude can be lowered by reducing the cruise speed. Less obviously, for a given cruise speed, the Reynolds number decreases with altitude, but with an associated increase in Mach number until the tropopause is reached at $11 \mathrm{~km}$. 


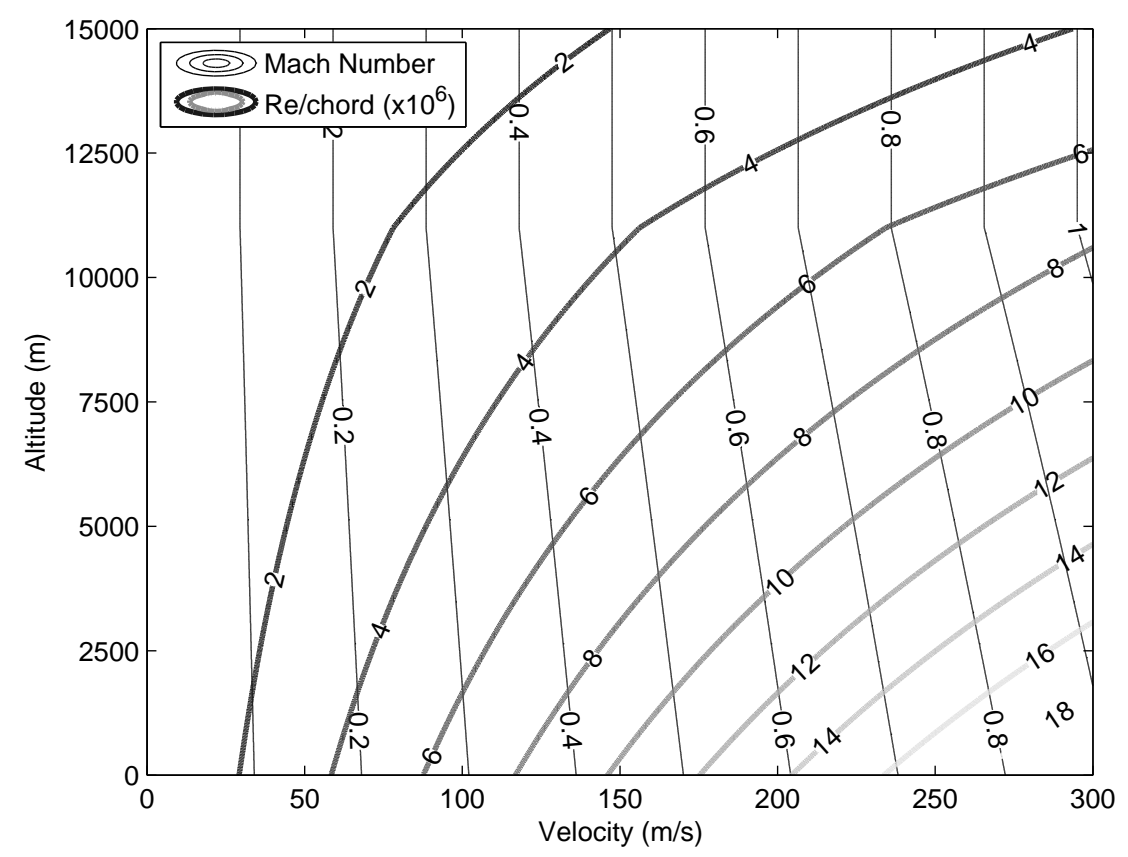

Figure 5.1: Altitude against velocity with contours of Mach number and unit Reynolds number.

In this section, the constraints and design variables that influence the choice of Mach number and unit Reynolds number are identified, and an explanation of how they are combined to produce a design specification is given. The constraints fall under two categories: geometric and aerodynamic.

\subsubsection{Aircraft Configuration and Geometric Constraints}

The architecture of the flying-wing aircraft is two-fold: the centrebody accommodates passengers, whilst the outer wing has a more conventional structure, providing volume for fuel tanks and cargo holds (see Chap. 2). For the sake of simplicity, a swept, untapered flying-wing planform, having chord $c$ and span $b$, is assumed here (see Fig. 5.2).

A cabin height of about $2 \mathrm{~m}$ must be provided for passengers to stand upright. A value of $2.5 \mathrm{~m}$ is therefore imposed on the (maximum) wing section thickness; the extra $0.5 \mathrm{~m}$ allows for the hardware necessary to suck and transport the air from the boundary layer, and for the wing structural reinforcements. It is immediately apparent that thickness-to-chord ratios comparable to current high-speed subsonic aircraft verge on the impractical - $10 \%$, for example, would require a chord of $25 \mathrm{~m}$ - and thicker sections may well be preferable. 
Therefore, the range $0.1 \leq t / c \leq 0.3$ is considered. The aerofoil section geometry will be dictated as much by passenger accommodation requirements as aerodynamic performance, and should have minimal nose-down pitching moment at zero lift (for trim reasons (Nickel and Wohlfart (1994), Donlan (1944), and Jones (1941))). Therefore, where section details are needed, the family of symmetrical (00) NACA 4-digit aerofoils will be assumed.

The large chord associated with the passenger accommodation constraint implies a correspondingly large wingspan. Existing airport ground-handling infrastructure requires that the aircraft planform fit within an $80 \mathrm{~m} \times 80 \mathrm{~m}$ box (Denning et al. (1997)). For possible span values, we consider $60 \mathrm{~m}, 80 \mathrm{~m}$ and $100 \mathrm{~m}$. The last is to quantify any advantages that might ultimately lead to a re-evaluation of current airport facilities.

Finally, note that elements providing directional stability and yaw control are not shown in Fig. 5.2. The use of 'winglet rudders' is envisaged, which are essentially vertical fins, with control surfaces, placed at the wing tips (see Chap. 2).

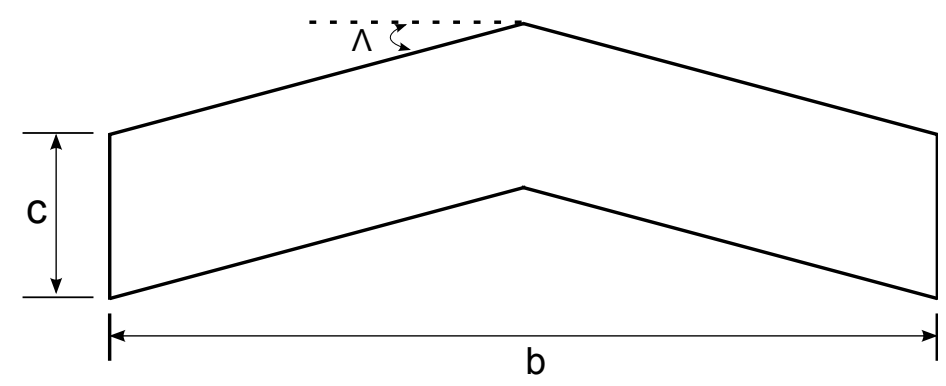

Figure 5.2: Schematic diagram of a simplified laminar-flying-wing planform layout.

\subsubsection{Aerodynamic Constraints}

\subsubsection{Unit Reynolds Number}

As previously discussed in Chap. 2, the maximum possible unit Reynolds number depends on the surface finish and atmospheric turbulence levels. Although a $R e_{u}$ up to $16 \times 10^{6} \mathrm{~m}^{-1}$ is realistically achievable for a two-dimensional aerofoil flow, values this high compromise the stability of the attachment-line boundary layer on swept wings (see below). Here, then, values of 4,8 and $12 \times 10^{6} \mathrm{~m}^{-1}$ are considered.

\subsubsection{Planform Sweep}

This study is restricted to LFW configurations for which the suction requirements can be calculated from consideration of two-dimensional, Tollmien-Schlichting instabilities only. Thus, to avoid cross-flow instabilities, the wing sweep angle must be limited. The dependence of 


\section{CHAPTER 5. LFW AIRCRAFT AND MISSION SPECIFICATION}

cross-flow instability on pressure distribution, discussed in Chap. 2, means that this limit is a function of the aerofoil section geometry. However, in light of Joslin (1998b) (see Chap. 2), a uniform value of $25^{\circ}$ should ensure that the suction flow is dominated by the need to stabilise Tollmien-Schlichting waves.

A further issue in swept-wing transition is the stability of the 'attachment-line' boundary layer on the leading edge (see Chap. 2). For the LFW, attention is restricted to natural transition on the attachment line. As the nose radius of NACA 4-digit aerofoils has a functional dependence on the maximum section thickness-to-chord ratio (Abbott and Von Doenhoff (1959)), Eq. 2.8 (repeated below) allows for evaluation of the maximum planform sweep angle consistent with a laminar attachment-line boundary layer. In this calculation, $R e_{a . l}$ is set to 650, at the centre of Poll's critical-value range.

$$
R e_{a . l}=\left(\frac{U_{\infty} r}{\nu} \times \frac{\sin \Lambda \tan \Lambda}{2}\right)^{\frac{1}{2}}
$$

\subsubsection{Mach Number}

In Chap. 2 problems arising due to a steep pressure rise through a shock wave associated with supercritical flow, and its adverse effect on the stability of the boundary layer, were discussed. Consequently, in this study, the design is restricted to the subcritical flow regime; i.e. the cruise Mach number is chosen to be equal to the critical Mach number, for a given wing sweep angle, section thickness-to-chord ratio and lift coefficient.

\subsubsection{Design Specification Algorithm}

An approach is developed whereby the aircraft/mission specification is defined on the basis of three key variables: unit Reynolds number, span and thickness-to-chord ratio. With the thickness already fixed, the chord length, planform aspect ratio and planform area follow directly from the span and thickness-to-chord ratio. The algorithm then proceeds as follows:

(a) the wing sweep is maximised, subject to the cross-flow limit and the attachment-line transition criterion;

(b) the aircraft drag polar is estimated;

(c) the requirement that the aircraft cruises at high efficiency is used to set the lift and drag coefficients;

(d) with lift coefficient and section geometry known, the critical Mach number is calculated; 
(e) the cruise Mach number and unit Reynolds number now set the cruise altitude and speed (c.f. Fig. 5.1). The allowable cruise weight, wing loading and lift-to-drag ratio follow directly from the cruise lift coefficient, drag coefficient and aircraft geometrical parameters, combined with the free-stream conditions.

The algorithm outlined above is performed over a range of thickness-to-chord ratios: first for the three specified spans and a fixed unit Reynolds number, and then three specified unit Reynolds numbers and a fixed span. Results from this process are presented in Sec 5.3. First, in the following section, details of the aerodynamic calculation methodology are given.

\subsection{Methodology}

\subsubsection{Drag Polar}

A drag polar of the form given in Eq. 5.1 is assumed, where the first and second terms are the zero-lift and lift-dependent drag respectively (Katz and Plotkin (2001)). (Martinez-Val and Perez (1991) comment that the errors involved in Eq. 5.1 are very small, since drag divergence is not an issue in long-range cruise.)

$$
C_{D}=C_{D 0}+K C_{L}^{2}
$$

On a conventional aircraft, the lift-dependent drag parameter, $K$, is typically slightly greater than the 'ideal', elliptical-lift-distribution value, $\left(\pi A_{R}\right)^{-1}$. In part, the excess is due to 'trim drag', which may, depending on the longitudinal stability margin, be more significant for a flying wing. On the other hand, the end-plate effect of winglet rudders will tend to reduce $K$. Therefore, in the absence of detailed quantitative information on these competing factors, it is taken equal to $\left(\pi A_{R}\right)^{-1}$.

An estimate for the zero-lift drag coefficient, $C_{D 0}$, is obtained by performing a drag calculation for the aerofoil section with zero lift and wing-normal Mach number 0.5. (This Mach number is close enough to the final expected cruise condition to avoid significant errors, whilst, in addition, guaranteeing subcritical flow for the full range of thickness-to-chord ratios considered.) Details of the calculation are given below.

It should be recognised that this approach falls some way short of a detailed drag build-up. In particular, contributions from the winglet rudders, the engine pylons, and the other sources classified by Torenbeek (1976) (and detailed in Chap. 3) as 'miscellaneous' are neglected. For this reason, sensitivity to errors in the estimated drag polar should be considered; this issue will be addressed in Sec. 5.4.3. 


\subsubsection{Cruise Condition}

At the optimum cruise condition, an aircraft's fuel consumption per unit distance is minimised. This requirement corresponds to maximising the range parameter $\left(U_{\infty} / s f c\right)(L / D)$, where $s f c$ (the specific fuel consumption) is, in general, a function of cruise speed and altitude, and depends on the type of powerplant used (Martinez-Val and Perez (1991)).

For an aircraft utilising suction, the thermal energy from the fuel is determined by the thrust power requirement and the power off-take necessary to energise the suction system:

$$
\dot{m}_{f} L C V=\frac{T U_{\infty}}{\eta_{0}}+\frac{D_{\text {pump }} U_{\infty}}{\eta_{\text {pump }} \eta_{t h}} .
$$

Adding the assumption that $\eta_{\text {pump }} \approx \eta_{p}$ then, since $\eta_{0}=\frac{1}{s f c} \frac{U_{\infty}}{L C V}$, Eq. 5.2 becomes

$$
\frac{\dot{m}_{f}}{U_{\infty}}=\frac{W}{L / D} \times \frac{s f c}{U_{\infty}}
$$

where $D=D_{\text {aero }}+D_{\text {pump }}$. This corresponds to the standard result, and suggests that it is robust to the inclusion of pump drag in $C_{D 0}$.

Martinez-Val and Perez (1991) note that the performance of turbofan engines lies between two theoretical idealisations: $s f c=$ constant (in which case one should maximise $U_{\infty} L / D$ ) and $s f c \propto U_{\infty}$ (maximise $\left.L / D\right)$. To avoid assuming specific propulsion characteristics at this stage, one of these conditions is chosen, accepting that it will probably be somewhat suboptimal. Given that either will imply unusually low wing loading (as will become apparent), with adverse implications for structural efficiency, the condition which mitigates this issue is opted for. This is the one corresponding to a higher lift coefficient, i.e. that of maximum $L / D$. The associated lift and drag coefficients follow from Eq. 5.1; they are:

$$
C_{L}=\sqrt{\pi C_{D 0} A_{R}} \text { and } C_{D}=2 C_{D 0}
$$

\subsubsection{Lift and Drag Calculations}

Sweep introduces three-dimensionality into the wing flow, as already discussed. For reasonably high aspect ratios, the flow over most of the wing is similar to that over a swept wing of infinite span (Houghton and Carpenter (1993)). Hileman et al. (2007) use infinite swept wing theory, as it greatly simplifies the aerodynamic calculations: it allows 3-D aircraft coefficients to be related to simple perpendicular 2-D aerofoil coefficients. However, its use here should be qualified by the observation that its predictions at the lowest aspect ratios considered in the parameter study that will follow are unlikely to be reliable. 
The flow in the plane normal to the spanwise axis has a reduced Mach number

$$
M_{\perp}=M_{\infty} \cos \Lambda
$$

however the effective thickness-to-chord ratio increases to

$$
(t / c)_{\perp}=\frac{(t / c)}{\cos \Lambda}
$$

If the 2-D lift coefficient for the normal-plane flow geometry is $C_{l_{\perp}}$, then the local lift coefficient (based on the free-stream velocity) is $C_{l_{\perp}} \cos ^{2} \Lambda$. For a well designed aircraft, this local lift coefficient will be closely representative of the overall, aircraft, lift coefficient, so

$$
C_{L}=C_{l_{\perp}} \cos ^{2} \Lambda
$$

The drag coefficient is determined following the considerations presented in Chap. 3 for a swept wing, which requires evaluation of the surface pressure, and hence velocity, distribution. For the present investigation XFoil is used. The other stage at which the wing pressure distribution is required, is for determining the critical Mach number at the cruise lift coefficient. Here the section lift coefficient is found via Eqs. 5.4 and 5.7; XFoil calculations at this lift coefficient are iterated numerically to find the Mach number at which $C_{p, \min }$ is equal to the sonic value. (Note that this approach does not guarantee fully subcritical flow on the centre-line, where the infinite swept-wing theory breaks down. On the other hand, the calculation is conservative in the sense that the NACA 4-digit geometry is not optimised for transonic flow; therefore, it may be argued that subcritical centre-line flow will be attainable with a suitable, bespoke, aerofoil section.)

Contributions to the zero-lift drag are two-fold: there is a viscous profile drag, which can be determined based on the momentum thickness at the trailing edge using the SquireYoung formula, given by Eq. 3.5; and a 'pump drag', due to the additional power that must be supplied by the engines to re-energise the sucked boundary layer fluid. In estimating the pump-drag contribution, reference is made to Chap. 4, wherein a minimum pump-power requirement independent of the suction system design was identified. It is associated with the boundary-layer stagnation pressure loss of the flow in an optimal suction distribution, and accounts for $80 \%-90 \%$ of the total power requirement; therefore, in this study, the pump drag is estimated based on the minimum power requirement alone. Furthermore, the Mach number and lift coefficient were found to have a negligible effect on the pump power requirement, compared to the influence of thickness-to-chord ratio, and are thus also discounted here. Finally, as full-chord laminarisation is realistically unachievable, given the 


\section{CHAPTER 5. LFW AIRCRAFT AND MISSION SPECIFICATION}

space requirements of the suction hardware and the presence of trailing-edge control surfaces, suction is assumed to be available over the first $90 \%$ of chord only. (Note, however, that it will typically not be required until some distance aft of the leading edge, as detailed in Chap. 4.)

\subsection{Aircraft and Mission Parametric Study}

The planform layout and architecture of the LFW, along with the key design constraints, have been identified. Moreover, three principal design variables have been proposed: maximum section thickness-to-chord ratio $(0.1 \leq t / c \leq 0.3)$, wing span $(60 \mathrm{~m}, 80 \mathrm{~m}$ and $100 \mathrm{~m})$ and unit Reynolds number $\left(4 \times 10^{6}, 8 \times 10^{6}\right.$ and $\left.12 \times 10^{6} \mathrm{~m}^{-1}\right)$. Once these variables have been set, the aircraft/mission specification follows from the algorithm of Sec. 5.1.3. At this stage, enough information is available to investigate further parameters, namely: lift-to-drag ratio, wing loading, take-off lift coefficient, and allowable weight.

\subsubsection{Aspect Ratio}

For the untapered swept-wing model assumed, the aspect ratio is simply given by $A_{R}=b / c$. Aspect ratio increases linearly with thickness-to-chord ratio, for a given span, because of the fixed thickness constraint. Over the range of spans and thickness-to-chord ratios considered, the aspect ratio varies from low values of around two, to much higher values near twelve.

\subsubsection{Sweep}

Figure 5.3 shows the variation of wing sweep with thickness-to-chord ratio and unit Reynolds number. (Equation 2.8 is independent of wing span.) For low unit Reynolds numbers, the assumed cross-flow limit of $25^{\circ}$ applies. As unit Reynolds number is increased, the influence of thickness-to-chord ratio on attachment-line stability becomes more important. For the highest value of unit Reynolds number, the reduction in sweep at the maximum thicknessto-chord ratio considered is approximately $8^{\circ}$.

\subsubsection{Profile Drag}

Figure 5.4 details the zero-lift drag breakdown over a range of maximum thickness-to-chord ratios and unit Reynolds numbers. As thickness-to-chord ratio is increased, the viscous drag shows a small reduction. (This is because the momentum thickness at the trailing-edge is almost independent of $t / c$, while the Squire-Young correction factor decreases slightly.) However, the effect of thickness on pump drag is far more significant, due to greater losses in dynamic pressure through the boundary layer, and increases in the required suction flow (see 


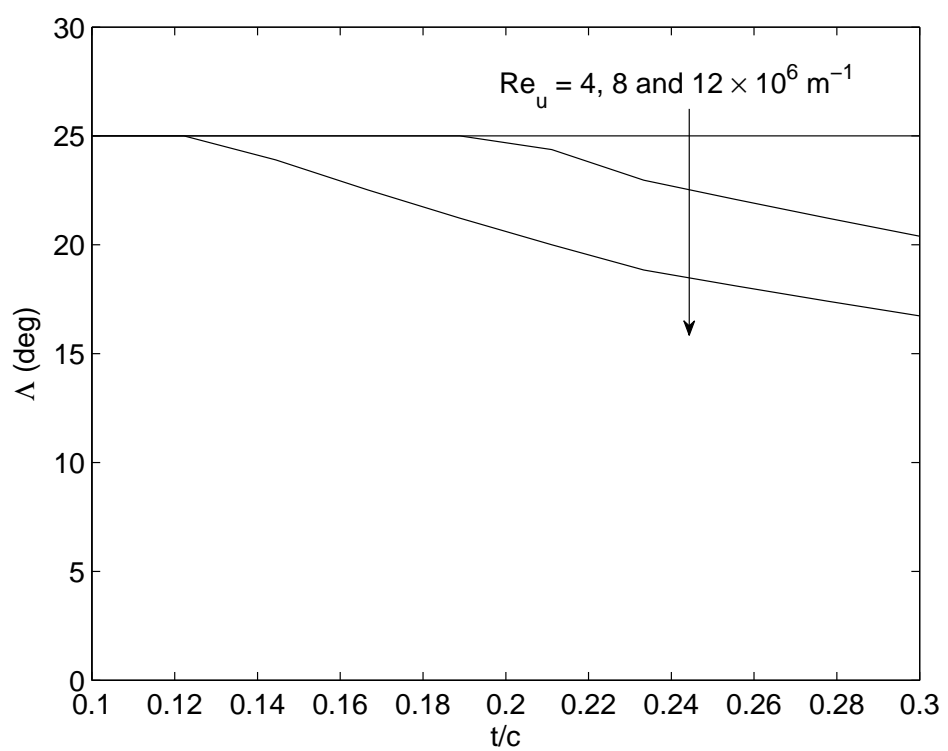

Figure 5.3: Wing sweep angle dependence on thickness-to-chord ratio and unit Reynolds number.

Chap. 4). The influence of unit Reynolds number is important for both components of drag: the momentum thickness at the trailing-edge reduces; whilst, for the pump drag, it is well established that the suction flow rate depends on chord Reynolds number as $C_{Q} \propto R e_{c}^{-1 / 2}$. In Chap. 4 it was found that the pump power coefficient, or equivalently the pump drag coefficient, is found to have a similar, albeit slightly weaker, dependence.

\subsubsection{Cruise Lift Coefficient and Aerodynamic Efficiency}

With the zero-lift drag known, the cruise lift coefficient can be evaluated; moreover, the variation of lift-to-drag ratio, $L / D$, can be determined. The results are detailed in Fig. 5.5. The cruise lift coefficient lies between approximately 0.1 and 0.2 across the entire parameter range. Such low values result directly from the small zero-lift drag contribution, which in turn requires a low induced drag for cruise at maximum $L / D$. The increase in lift coefficient with thickness-to-chord ratio is due to the rise in both zero-lift drag coefficient and aspect ratio (see Eq. 5.4). For a given thickness-to-chord ratio, increasing span, and hence aspect ratio, increases the lift coefficient. For fixed span and thickness-to-chord ratio, lift coefficient drops with unit Reynolds number, due solely to the reduction in the zero-lift drag coefficient; however, the influence is weak as, from Eq. 5.4, $C_{L} \propto \sqrt{C_{D 0}}$, and the $C_{D 0}$ dependence itself is less than $R e^{-1 / 2}$. 


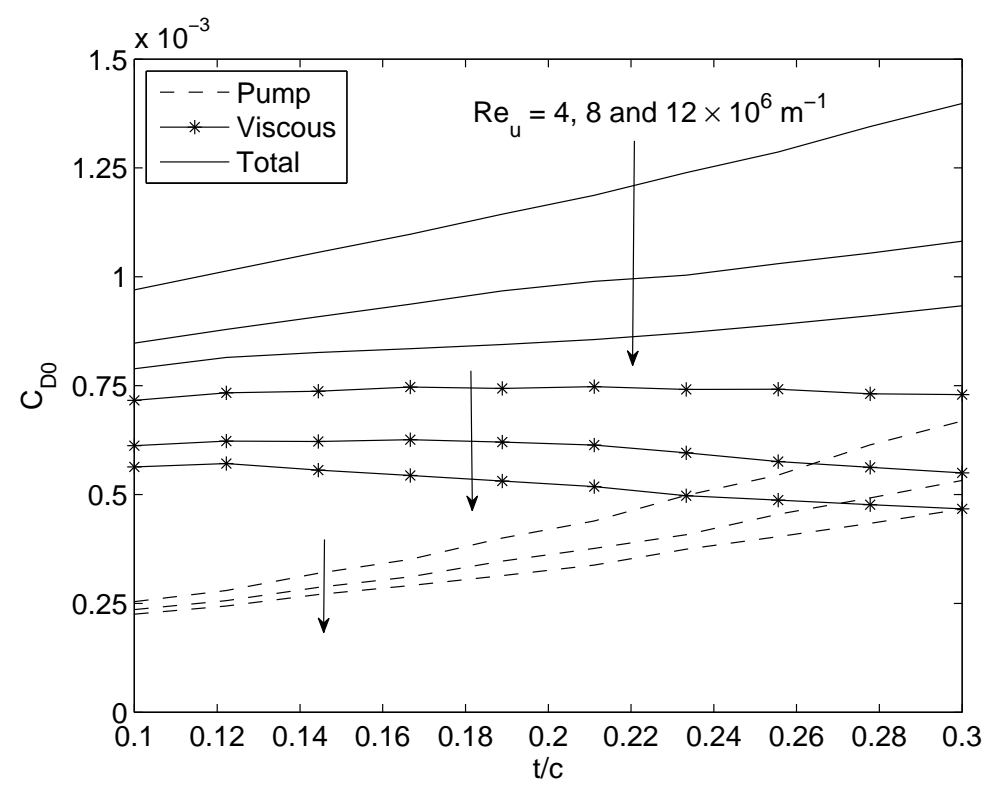

Figure 5.4: Zero-lift drag breakdown and its dependence on thickness-to-chord ratio and unit Reynolds number.

Due to the low zero-lift drag expected for LFW aircraft, we see from Fig. 5.5 that a lift-to-drag ratio of around $50-90$ is predicted. Again from Eq. 5.4, $L / D \propto \sqrt{A_{R} / C_{D 0}}$. Consequently, the increase in $L / D$ with $t / c$ arises because the aspect ratio rises faster than $C_{D 0}$. Furthermore, for fixed $t / c$, increases in span improve $L / D$ via the aspect ratio term. Finally, the lift-to-drag ratio also improves with unit Reynolds number, but this time due to the reduction in the zero-lift drag coefficient. 

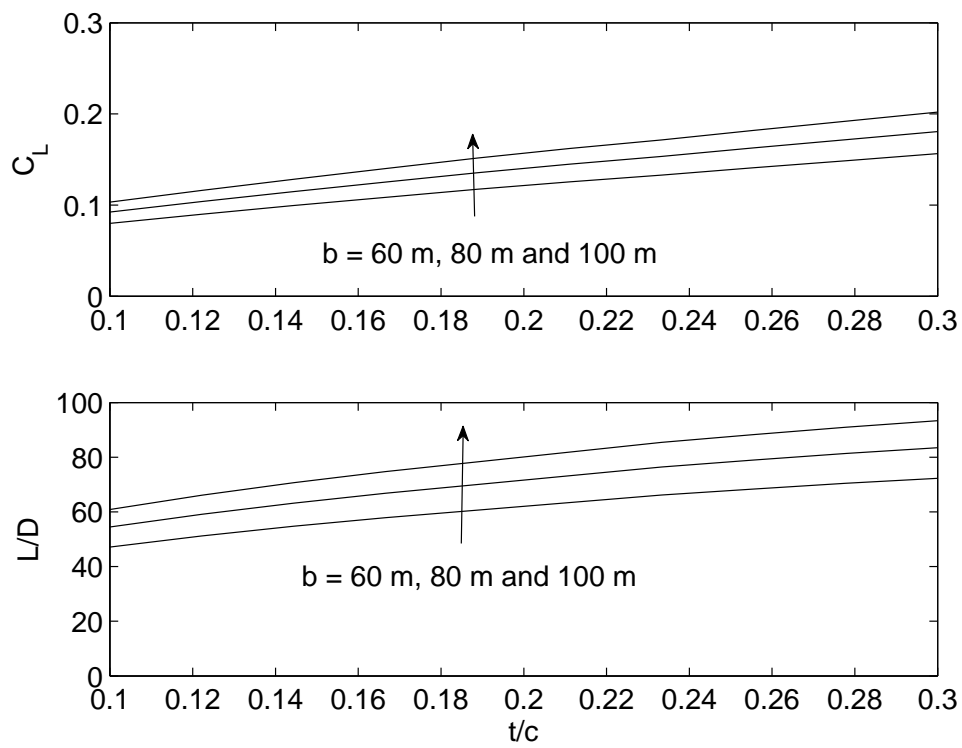

(a)
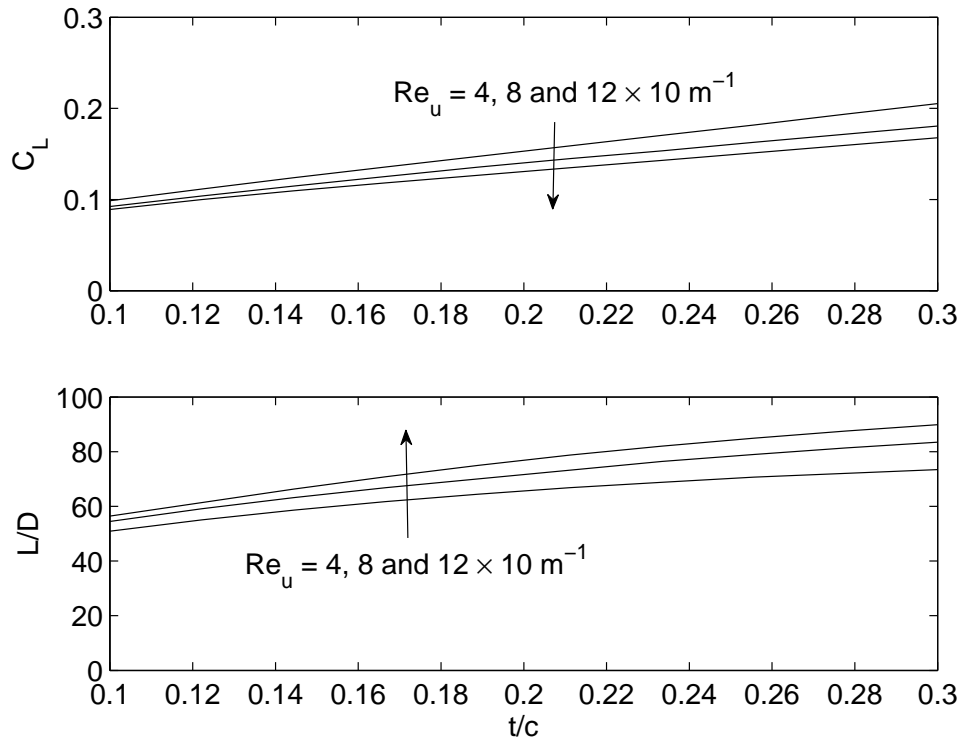

(b)

Figure 5.5: The dependence of cruise lift coefficient and lift-to-drag ratio on thickness-tochord ratio for: (a) a range of spans and a fixed $R e_{u}$ of $8 \times 10^{6} \mathrm{~m}^{-1}$ and, (b) a range of unit Reynolds numbers and a fixed span of $80 \mathrm{~m}$. 


\subsubsection{Cruise Mach Number}

With the dependence of lift coefficient on the three design variables evaluated, the critical Mach number, and hence cruise Mach number, can be calculated via the approach outlined in Sec. 5.2.3. The cruise Mach number is shown as a function of thickness-to-chord ratio, span and unit Reynolds number in Fig. 5.6. Although inviscid flow is assumed in the calculation, a small unit Reynolds number dependence arises due to the influence of zero-lift drag on cruise lift coefficient. As expected, cruise Mach number reduces with increasing thickness-to-chord ratio; span and unit Reynolds number variations have very little effect because the maximum local Mach number is insensitive to lift coefficient changes at the low values found here. (The same observation was made by Lee (1961) in the HP117 proposal.)

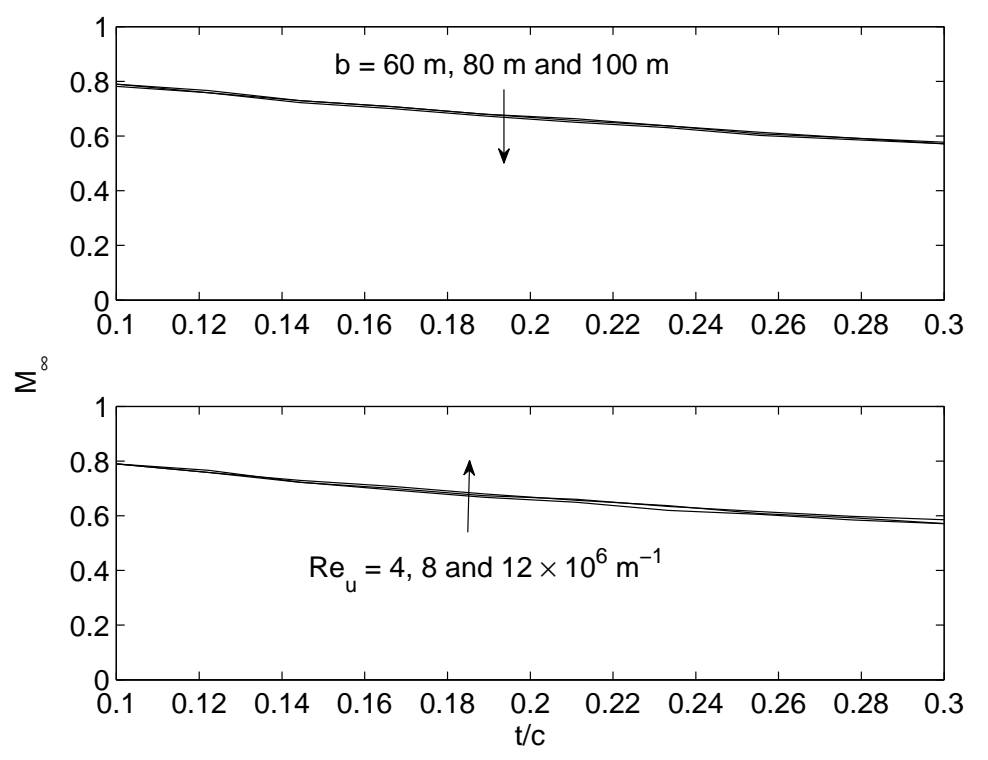

Figure 5.6: Cruise Mach number dependence on thickness-to-chord ratio, span and unit Reynolds number. (Top figure corresponds to a $R e_{u}=8 \times 10^{6} \mathrm{~m}^{-1}$, and the bottom to a span of $80 \mathrm{~m}$.

\subsubsection{Cruise Altitude and Velocity}

The calculations needed to specify the cruising altitude and speed are now complete. Figure 5.7 details the variation of these two parameters with thickness-to-chord, span and unit Reynolds number.

We see in Fig. 5.7 (a) that, as thickness-to-chord ratio increases for a fixed unit Reynolds 

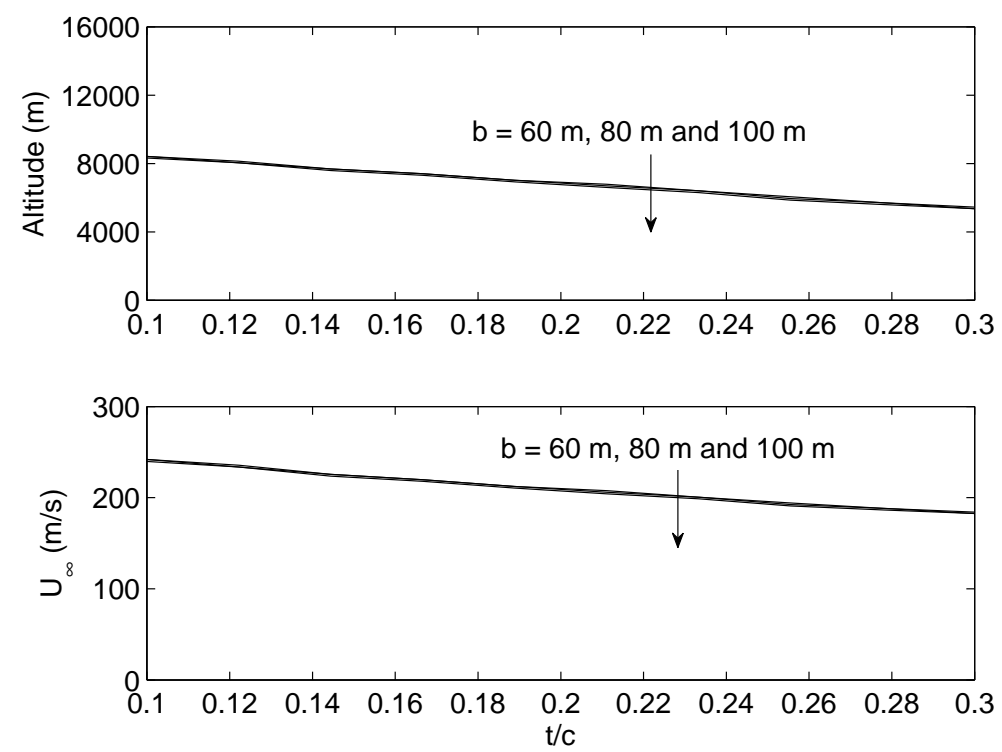

(a)
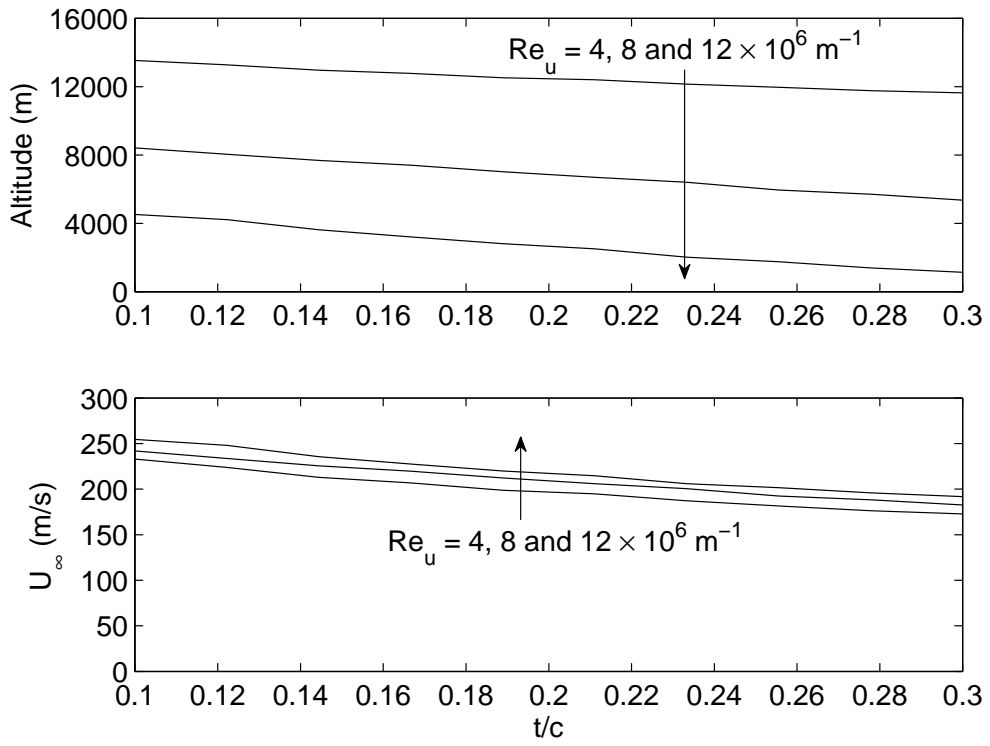

(b)

Figure 5.7: The dependence of cruise altitude and cruise velocity on thickness-to-chord ratio for: (a) a range of spans and a fixed $R e_{u}$ of $8 \times 10^{6} \mathrm{~m}^{-1}$ and, (b) a range of unit Reynolds numbers and a fixed span of $80 \mathrm{~m}$. 


\section{CHAPTER 5. LFW AIRCRAFT AND MISSION SPECIFICATION}

number, the cruise Mach number drops and, hence, so do cruise altitude and velocity. At fixed $t / c$ (and $R e_{u}$ ), span has a negligible influence on cruise Mach number, and hence also altitude and velocity. Unsurprisingly, the influence of $R e_{u}$ is far more significant, as shown in Fig. 5.7 (b). With the dependence of cruise Mach number on unit Reynolds number already seen to be negligible, increases in the latter lead directly to reductions in cruise altitude (c.f. Fig. 5.1). The associated increases in ambient temperature result in slightly higher cruise velocities.

\subsubsection{Wing Loading and Maximum Lift Coefficient}

For conventional aircraft designs, Jenkinson et al. (1999) comment that, typically, takeoff and landing requirements ultimately dictate the planform area, and hence wing loading. In contrast, here, the requirement to cruise at maximum $L / D$, and the need to maintain laminar flow, fixes the cruise altitude, velocity and lift coefficient, which in turn set the cruise wing loading, as:

$$
w_{L}=\frac{1}{2} \rho_{\infty} U_{\infty}^{2} C_{L} .
$$

In Fig. 5.8, wing loading is shown to increase with thickness-to-chord ratio; despite the associated reduction in cruise velocity, the drop in altitude (and hence increase in atmospheric density) and increase in lift coefficient dominate. A slight dependence on span follows directly from the influence of lift coefficient. In contrast, we can see that unit Reynolds number has a far greater impact. Due to the significant reduction in altitude associated with an increase in unit Reynolds number, the increments in wing loading are substantial. Nonetheless, even the highest values in Fig. 5.8 barely exceed half of a conventional aircraft's wing loading. Such low figures raise the prospect of an unacceptably high structural-weight fraction; this issue is therefore explored further in the following section.

For conventional aircraft, high-lift devices are necessary to achieve the lift coefficients needed for takeoff and landing (Jenkinson et al. (1999)). The maximum lift coefficient is related to wing loading by

$$
C_{L, \text { max }}=\frac{w_{L}}{\frac{1}{2} \rho_{\text {sea }} U_{\text {stall }}^{2}},
$$

where $U_{\text {stall }}$ is the stalling speed. Despite the low wing loading expected of a LFW aircraft, the maximum lift coefficient is of concern as high-lift devices cannot be utilised (Martinez-Val et al. (2006)); therefore, it is necessary to check that the design does not implicitly assume one that is too great.

To evaluate Eq. 5.9, an initial estimate of the stall velocity is based on that of a conven- 


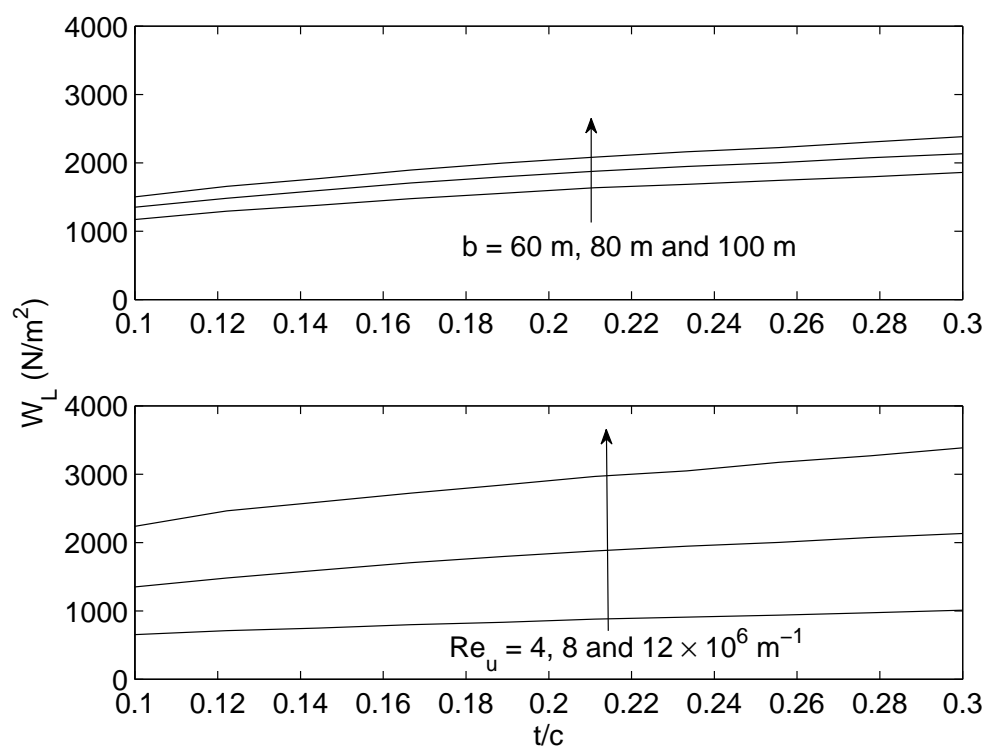

Figure 5.8: Variation of aircraft wing loading with thickness-to-chord ratio, span and unit Reynolds number. (Top figure corresponds to $R e_{u}=8 \times 10^{6} \mathrm{~m}^{-1}$, and the bottom to a span of $80 \mathrm{~m}$.

tional aircraft. For a Boeing 737-200 in takeoff configuration, with ${ }^{1} C_{L, \max }=2.07$ and $w_{L}=$ $5040 \mathrm{~N} / \mathrm{m}^{2}$, the stalling speed is $63.7 \mathrm{~m} / \mathrm{s}$. The LFW maximum lift coefficient corresponding to this value is shown in Fig. 5.9. As expected from Eq. 5.9, $C_{L, \max }$ has the same dependence on the three design variables as wing loading. It lies below 1.4, a value attainable without high-lift devices, over the entire range of parameters considered. Therefore, in conclusion, $C_{L, \max }$ is unlikely to constrain a LFW design in the way it does for conventional aircraft.

\subsubsection{Maximum Cruise Weight and Structural Weight Fraction}

In a conventional design process, the gross aircraft weight is estimated on the basis of a detailed mission specification. In contrast, here the allowable weight of the aircraft in cruise may simply be found from the cruise lift coefficient, as $\frac{1}{2} \rho_{\infty} U_{\infty}^{2} b c C_{L}$. This value then becomes one of the constraints which will be passed to the conceptual design study in Chap. 6 .

Figure 5.10 shows that, for the range of parameters considered here, the proposed LFW could sustain a gross weight anywhere between 75 and 460 tonnes (mt). The figure shows a reduction in allowable weight with thickness-to-chord ratio. This is because $U_{\infty}^{2} c$ drops at a faster rate than $\rho_{\infty} C_{L}$ increases. As span increases for a given $t / c$, the allowable weight

\footnotetext{
${ }^{1}$ www.b737.org.uk/techspecsdetailed.htm, October 2011
} 


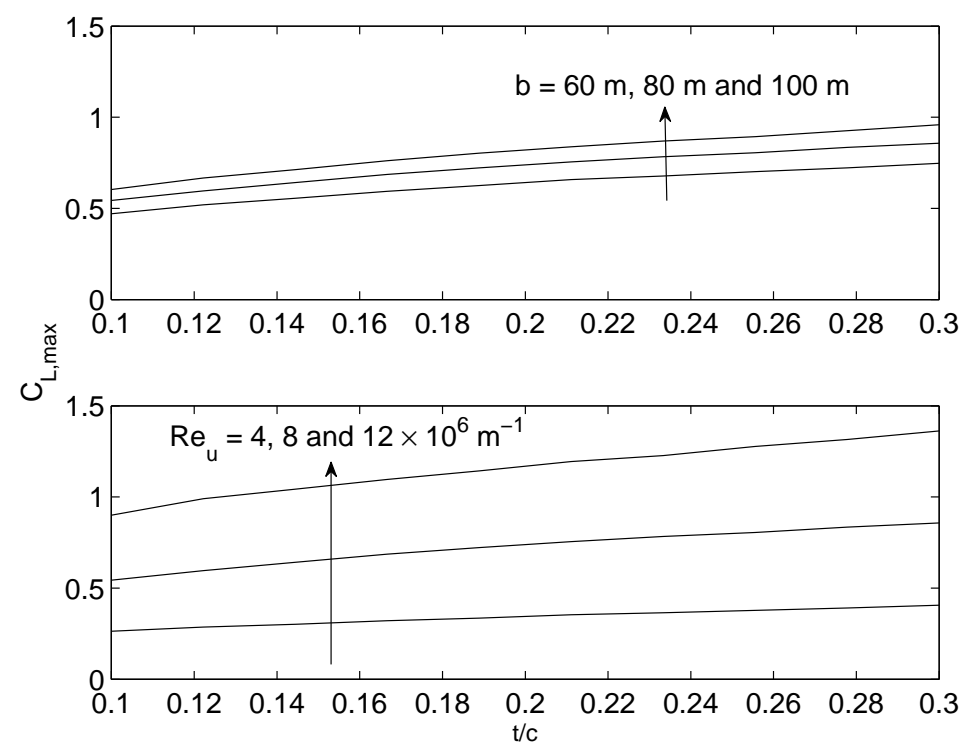

Figure 5.9: Variation of required maximum lift coefficient with thickness-to-chord ratio, span and unit Reynolds number. (Top figure corresponds to a $R e_{u}=8 \times 10^{6} \mathrm{~m}^{-1}$, and the bottom to a span of $80 \mathrm{~m}$.)

rises, as expected, given the increase in planform area. Note, however, that the improvement in wing loading evident in Fig. 5.8 also has a significant effect. As for the influence of unit Reynolds number, we see that the increased density and cruise velocity associated with the reduction in cruise altitude vastly outweigh the effect of a slightly lower lift coefficient.

Perhaps more important than the overall weight is the structural-weight fraction, as it gives an indication of the aircraft's payload capability. The structure of the centrebody pressure vessel will be of an unconventional nature (see Chap. 2), and an estimate of its weight is beyond the scope of the present investigation. Nevertheless, the wing weight serves as a useful indicator of the overall structural weight.

Since the wing structure of a LFW aircraft can be designed using conventional methods, Raymer (1999) provides some guidance as to its weight through an empirical correlation for cargo/transport aircraft (American units):

$$
W_{w}=0.0051\left(W N_{z}\right)^{0.557} S_{w}^{0.649} A_{R}^{0.5}(t / c)^{-0.4}(1+\lambda)^{0.1}(\cos \Lambda)^{-1} S_{c s}^{0.1}
$$

Here the ultimate load factor $N_{z}=1.5 \times$ limit load factor (the limit load factor is taken as 2.5 ), the control surface area $S_{c s}$ is assumed to be $10 \%$ of the total wing area $S_{w}$, and the taper ratio $\lambda=1$. 


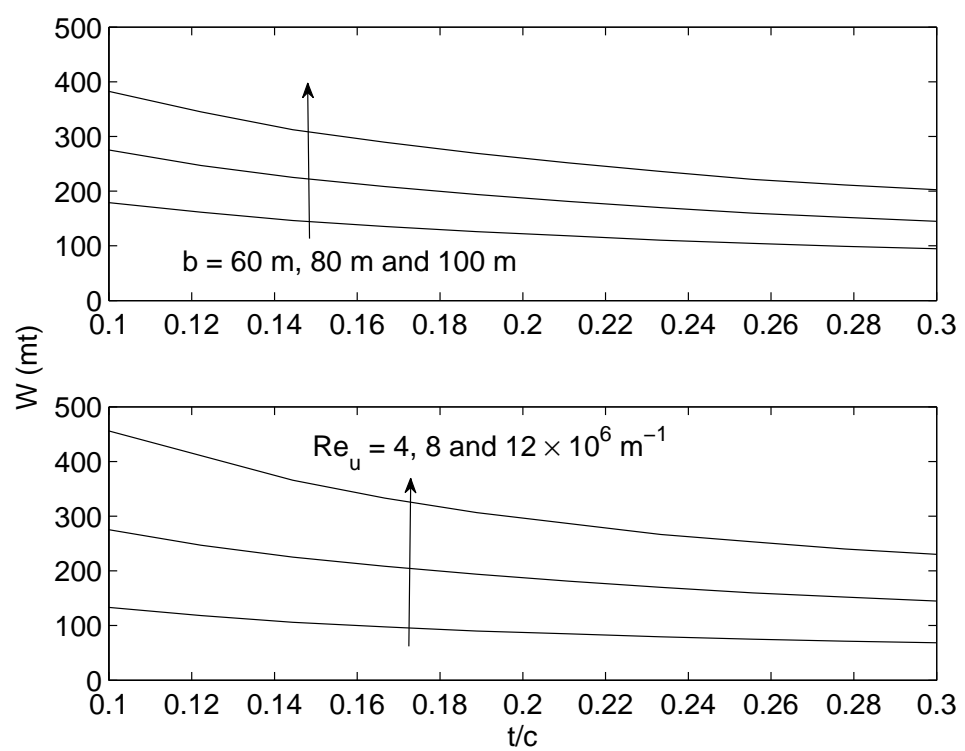

Figure 5.10: Variation of aircraft allowable weight with thickness-to-chord ratio, span and unit Reynolds number. (Top figure corresponds to a $R e_{u}=8 \times 10^{6} \mathrm{~m}^{-1}$, and the bottom to a span of $80 \mathrm{~m}$.

Further insight is provided through rearrangement of Eq. 5.10 into a form that gives the wing-weight fraction for a given thickness and sweep as a function of wing loading, span and maximum thickness-to-chord ratio,

$$
\frac{W_{w}}{W} \propto w_{L}^{-0.443} b^{0.806}(t / c)^{-0.206} .
$$

Equation 5.11 reveals that the wing-weight fraction has an inverse relationship with both wing loading and thickness-to-chord ratio, whilst it increases with span. Recall that, from Fig. 5.8, $w_{L}$ increases with $t / c, b$ and $R e_{u}$.

Figure 5.11 details the calculated variation of the wing-weight fraction with thickness-tochord ratio, span and unit Reynolds number. Here we see first the expected reduction with $t / c$. Furthermore, as a consequence of increased wing loading, the wing-weight fraction drops when unit Reynolds number rises (at fixed $t / c$ ). However, although the dependence of $w_{L}$ on $b$ tends to reduce $W_{w} / W$, Fig. 5.11 shows that there is an overall increase with span as the direct dependence is stronger - this is confirmed by Eq. 5.11.

Finally, note that the absolute values of wing-weight fraction shown in Fig. 5.11 are unlikely to be strictly accurate; although designable with conventional methods, the LFW will be subject to a different loading distribution than its conventional counterpart. However, 


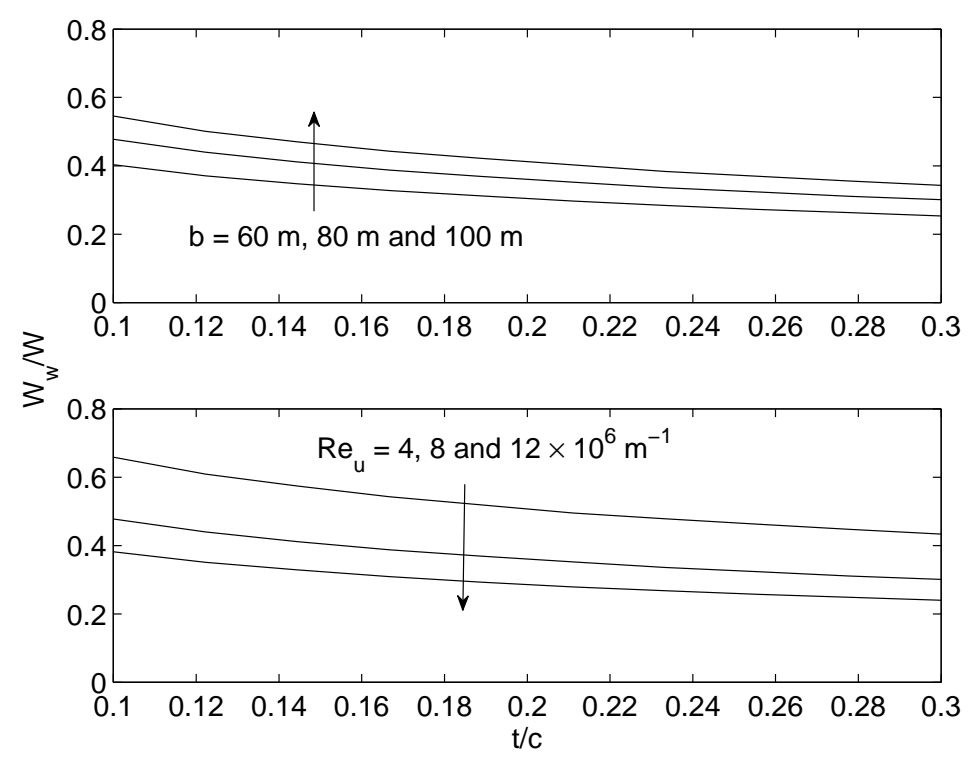

Figure 5.11: Variation of wing-weight fraction with thickness-to-chord ratio, span and unit Reynolds number. (Top figure corresponds to a $R e_{u}=8 \times 10^{6} \mathrm{~m}^{-1}$, and the bottom to a span of $80 \mathrm{~m}$.

the qualitative trends noted here will remain.

\subsection{Discussion}

In this section a feasible LFW design specification is determined by assessing trade-offs in the aircraft and mission parameters introduced in Sec. 5.3. The sensitivity of the chosen specification to potential errors in parameter estimates is discussed. Finally, key differences between the proposed LFW specification and conventional aircraft are highlighted.

\subsubsection{Mission Parameter Conflicts}

First consider the influence of increasing thickness-to-chord ratio. Depending on the value of the unit Reynolds number, there is a reduction in the allowable wing sweep; this conflicts with balance and stability requirements, which, as we learned in Chap. 2, are likely to require as much sweep as possible. There is a beneficial influence on the aerodynamic efficiency. The cruise speed reduces, which may reduce the LFW's attractiveness to airlines and passengers. The cruise altitude decreases, which not only avoids the undesirable emission of combustion products at high altitudes, but will also lead to a lighter cabin pressure vessel. The gust 


\subsection{DISCUSSION}

performance will improve with the associated increase in wing loading (Jenkinson et al. (1999)). Although the gross allowable weight decreases, so does the wing-weight fraction. However, as passenger-comfort requirements constrain the outboard extent of the cabin, the shorter chord at higher values of $t / c$ may limit passenger capacity.

Now consider the influence of increasing unit Reynolds number. The wing sweep may have to be reduced appreciably, again raising the prospect of balance and stability problems. The aerodynamic efficiency increases. The cruise altitude reduces sharply which, although generally beneficial (see above), could lead to adverse weather issues at the lower extremes. Two further gains are an increase in wing loading and a reduction in wing-weight fraction.

Finally, the benefits of an increase in span are two-fold: the aerodynamic efficiency and wing loading increase. However, we must bear in mind the penalty incurred in wing-weight fraction.

\subsubsection{Synthesis of Parameter Considerations}

To synthesise the parameter considerations discussed above, it is helpful to consider what combination of design variables leads to a high aerodynamic efficiency, without regard for other factors. The attainment of optimum aerodynamic efficiency is achieved through specification of the highest values of thickness-to-chord ratio, unit Reynolds number and span. This approach would also maximise wing loading, and hence minimise gust response issues.

The parameter conflicts discussed above reveal that high $t / c$ and $R e_{u}$ limit the degree of allowable sweep-back, and give rise to balance and stability issues; whilst the cruise speed is limited by high thickness-to-chord ratios. Other potential problems include: the wingweight fraction, which is driven to higher values with $b$ (though high $t / c$ and $R e_{u}$ have a favourable influence); impractically low cruise altitudes at the highest value of $R e_{u}$; and passenger capacity, which may be compromised at higher thickness-to-chord ratios.

These considerations lead to the conclusion that, to attain a viable design, values below maximum for all three parameters must be chosen. Therefore unit Reynolds number and span are fixed at $8 \times 10^{6} \mathrm{~m}^{-1}$ and $80 \mathrm{~m}$, respectively, as these give rise to a reasonable range of cruise altitudes and wing-weight fractions over the entire thickness-to-chord range considered here. At this value of unit Reynolds number, thickness-to-chord ratio is fixed at $20 \%$, because for higher values the allowable wing-sweep angle begins to fall sharply. The resulting aircraft and mission parameters are summarised in Tab. 5.1. 


\section{CHAPTER 5. LFW AIRCRAFT AND MISSION SPECIFICATION}

Table 5.1: Laminar-flying-wing aircraft design specification.

\begin{tabular}{lc}
\hline \hline Maximum thickness $(\mathrm{m})$ & 2.5 \\
Thickness-to-chord ratio & 0.20 \\
Span $(\mathrm{m})$ & 80 \\
Unit Reynolds number $\left(\mathrm{m}^{-1}\right)$ & $8 \times 10^{6}$ \\
Sweep (degrees) & 25 \\
Chord (m) & 12.5 \\
Planform area $\left(\mathrm{m}^{2}\right)$ & 1000 \\
Aspect ratio & 6.4 \\
Cruise lift coefficient & 0.14 \\
Mach number & 0.67 \\
Altitude $(\mathrm{ft})$ & 22500 \\
Velocity $(\mathrm{m} / \mathrm{s})$ & 209 \\
Allowable weight $(\mathrm{kg})$ & $187 \times 10^{3}$ \\
Wing loading $\left(\mathrm{N} / \mathrm{m}^{2}\right)$ & 1835 \\
\hline \hline
\end{tabular}

\subsubsection{Sensitivity to Error}

Having chosen a design specification, its robustness can now be considered. The potentially contentious aspects of the approach in this study are the estimates of the drag polar parameters, $C_{D 0}$ and $K$ (c.f. Eq. 5.1). Of these, $K$ is the more reliable; uncertainties over the competing influences of a non-ideal lift distribution and winglet end-plate effects are unlikely to cause errors significantly in excess of $10 \%$. In contrast, the pump-drag contribution to $C_{D 0}$ is heavily dependent on the neglect of suction requirements for the suppression of cross-flow instabilities, and the overall zero-lift drag value (below 0.001) is so low that it could be significantly increased by excrescence drag contributions. Consider, then, the effect of a doubling in this parameter on the design specification at given values of $b, t / c$ and $R e_{u}$. The cruise lift coefficient will increase by a factor $\sqrt{2}$, and the drag coefficient by a factor 2 . However, given the extremely weak dependence of cruise Mach number on $C_{L}$, the cruise speed and altitude will be essentially unchanged. The parameters which will alter significantly are the wing loading, allowable weight and structural efficiency, all of which will improve, and the aerodynamic efficiency, which will deteriorate (the lift-to-drag ratio drops to 51). The effect on appropriate values of $b, t / c$ and $R e_{u}$ will be only to make the largest span more attractive than previously. However, the associated trade-off between aerodynamic and structural efficiency would remain unclear, whereas there is a concrete argument (on existing airport infrastructure grounds) against increasing $b$ beyond $80 \mathrm{~m}$.

Alternatively, consider the effect of the same change in $C_{D 0}$ if all the parameters in 


\subsection{DISCUSSION}

Tab. 5.1 are held constant. (This corresponds to moving the cruise condition away from maximum $L / D$, and towards maximum $U_{\infty} L / D$.) The lift-to-drag ratio then drops by a factor $2 / 3$ to 48 , just $6 \%$ below its (new) maximum value.

In conclusion, the key features of the proposed specification remain justifiable in the face of potential errors in the zero-lift drag coefficient. The elements of Tab. 5.1 which may change as a result of an improved $C_{D 0}$ estimate are the cruise lift coefficient, allowable weight and wing loading; however, the level of uncertainty over their values does not undermine the usefulness of this specification as a basis for a conceptual design study.

\subsubsection{Comparison with Conventional Aircraft}

The initial constraints of laminar flow, limited sweep and $2.5 \mathrm{~m}$ thickness imply a design space that differs considerably from that inhabited by conventional aircraft. To attain the full benefit from the viscous drag reduction associated with laminarisation, the induced drag must also be small, which in turn leads to operation at a lift coefficient of 0.14 , with a correspondingly low wing loading of $1835 \mathrm{~N} / \mathrm{m}^{2}$. (To put these values into perspective, the Boeing 737-200 aircraft has a wing loading of $5040 \mathrm{~N} / \mathrm{m}^{2}$, and current aircraft typically cruise at a $C_{L}$ of around 0.5 (Cumpsty (1997)).) Under these conditions, the lift-to-drag ratio could be as high as 72, well above values for current, fully turbulent, jet transports, which are around $15-20$ (Cumpsty (1997)). Gust response, however, is a potential concern.

In spite of the low cruise $C_{L}$, the gross allowable weight is of the same order as conventional aircraft, at around $187 \mathrm{mt}$. (In comparison, the small-to-medium range Boeing 737-200 and the long-range Boeing 747-100 have gross weights of approximately $45 \mathrm{mt}$ and $322 \mathrm{mt}$, respectively (Torenbeek (1976)).) This is due to the large planform area expected of the LFW, and the reduced cruise altitude of $22,500 \mathrm{ft}$, relative to a current typical level around $35,000 \mathrm{ft}$.

Compared to conventional aircraft, the LFW is likely to have a high structural-weight fraction. Although the value for wing weight given by Eq. 5.10, around $40 \%$ of total weight, is unlikely to be very accurate, it hugely exceeds the $11 \%$ and $12 \%$ of the Boeing $737-200$ and 747-100 respectively (Torenbeek (1976)). Furthermore, it must be borne in mind that the flying-wing planform cannot accommodate a conventional cylindrical pressure vessel. Instead, non-cylindrical pressure vessels have been proposed; however, due to additional bending moment stresses, their weights may be excessive. Recall from Chap. 2 that, for this reason, other authors have suggested that multi-bubble configurations are preferable. On the positive side, the reduced cruise altitude should go some way towards alleviating this additional weight contribution. 


\section{CHAPTER 5. LFW AIRCRAFT AND MISSION SPECIFICATION}

Assuming take-off speeds similar to conventional aircraft, as done in this study, the takeoff lift coefficient is likely to be well below the maximum section lift coefficient. This may be exploitable to reduce the required airport runway length (which is often an issue for conventional long-range aircraft), by increasing the take-off $C_{L}$ and reducing the take-off speed. It also differentiates the LFW from the conventional design, whose wing area is largely determined by the maximum achievable lift coefficient, and which thus requires sophisticated high-lift devices.

Due to the imposed limitations on sweep angle, the requirement to maintain subcritical flow, and the fixed cabin height (which results in thick aerofoil sections), the cruise Mach number is reduced (relative to current long-range aircraft values of $0.8-0.85$ ) to 0.67 . However, an obvious advantage of cruising at a lower Mach number is that more efficient, open-rotor engine designs become an option (Martinez-Val et al. (2006)). It should also be noted that the proportionate drop in cruising speed is not as great as that in Mach number, due to the lower cruising altitude; the LFW here flies at $209 \mathrm{~m} / \mathrm{s}$, compared to $237 \mathrm{~m} / \mathrm{s}$ for a Mach 0.8 aircraft at $35,000 \mathrm{ft}$. The $16 \%$ Mach number reduction thus translates into a loss of only $12 \%$ in speed.

Finally, flying-wing aircraft differ from their conventional counterparts in the absence of a tail, and their longitudinal static stability margin is thus crucially dependent on sweep (see Chap. 2). As a result, while the value of $25^{\circ}$ specified here is not especially low by conventional standards, it may imply a need for artificial stabilisation via the flight control system.

\subsection{Summary}

In this chapter, the configuration and mission specification for a laminar-flying-wing aircraft with limited sweep have been studied. It has been assumed that the restriction on sweep $\left(25^{\circ}\right)$ allows suction requirements for the suppression of cross-flow instabilities to be neglected. Two further fundamental constraints have been imposed: it must be possible to stand in the cabin, and the flow must remain subcritical.

By treating the aircraft as an untapered wing, the design space is defined via three principal variables: span, thickness-to-chord ratio and unit Reynolds number. Suitable values for these parameters were found to be: $b=80 \mathrm{~m}, t / c=0.20$ and $R e_{u}=8 \times 10^{6} \mathrm{~m}^{-1}$. The key elements of the design are: a planform with area $1000 \mathrm{~m}^{2}$, chord $12.5 \mathrm{~m}$ and sweep $25^{\circ}$; a gross allowable weight of $187 \mathrm{mt}$, with a cruise Mach number of 0.67 at an altitude of 22,500 $\mathrm{ft}$; a provisional lift-to-drag ratio estimate in the range $48-72$, assuming laminar flow is maintained over $90 \%$ of the wetted area. 
To attain the full benefit of the viscous drag reduction, the induced drag must also be reduced, which in turn drives the LFW to a low cruise lift coefficient of 0.14 and low wing loading of $1835 \mathrm{~N} / \mathrm{m}^{2}$. Consequences of the low wing loading condition include potential gust response problems and a higher than conventional structural weight fraction; however, it implies that take-off does not constrain the aerodynamic design (in contrast to conventional aircraft). The combination of reduced sweep and high thickness-to-chord ratio is the cause of the lower than conventional cruise Mach number; taking altitude reduction into account, the LFW would fly about $12 \%$ slower than conventional aircraft. While potentially detrimental for passenger acceptance, this reduction opens up the prospect of using more efficient, open rotor, engines instead of standard turbofans. In addition, the low cruise altitude has beneficial implications for the impact of the aircraft's non- $\mathrm{CO}_{2}$ emissions.

A robust calculation of the fuel efficiency will follow from the conceptual design study, in Chap. 6, on the basis of this specification. However, a conservative initial estimate can be obtained as follows. Suppose that the empty weight of the aircraft is so high that it can carry only just over $10 \%$ of its gross weight (20 mt) as flight fuel, and the same amount as payload (say 200 passengers, with baggage, at $100 \mathrm{~kg}$ each). Then, for a lift-to-drag ratio of 50, the overall propulsion system efficiency, $\eta_{0}$, need only be $27 \%$ for the Breguet range equation, given by Eq. 3.84, to yield a flight distance of $6700 \mathrm{~km}$, corresponding to a fuel burn of 15 $\mathrm{g} /$ pax.km. This figure compares favourably with the value of $23.5 \mathrm{~g} /$ pax.km quoted for the Boeing 777, confirming (subject to a number of fundamental assumptions) the potential of the laminar-flying-wing configuration proposed here. 


\section{CHAPTER 6}

\section{Conceptual Design Study}

On the basis of the design specification given in Chap. 5, a detailed conceptual design of the LFW is developed in this chapter, following the design methodology formulated in Chap. 3, to assess the potential fuel-burn advantage over conventional aircraft. The specific objectives are to: generate a three-dimensional airframe on the basis of attaining satisfactory stability and control characteristics, and passenger accommodation; assess the cruise and low-speed performance of the aircraft; integrate the suction-system technology; design an engine that is capable of providing both the required thrust and suction power off-take; carry out an evaluation of the structure and its weight; and finally, determine the aircraft mission, and its fuel burn. For a fair comparison, the conceptual design of a baseline-turbulent aircraft for the same mission (range, payload, Mach number) and propulsion characteristics is also undertaken.

\subsection{Laminar-Flying-Wing Design Concept}

In Chap. 3 the overall design process for a LFW aircraft was outlined. In this section, the five design areas identified are covered in the following order: 1) 3-D airframe generation, 2) aircraft performance analysis, 3) suction-system technology integration, 4) propulsion system design, and 5) structures and weights. An overview of the key design constraints, inputs and outputs is provided for each area. Design methodologies specific to the LFW are also detailed. On attainment of a viable design, the aircraft's mission and fuel-burn performance are determined.

\subsubsection{Three-Dimensional Airframe Generation}

Figure 3.1 shows that the airframe influences four aspects of the design: the suction system via the aerofoil section geometry, the structure and its weight, the aircraft's performance, 
and the payload capacity. It consists of three main parts: the planform layout, and wing and cabin geometry. The planform and wing section geometries are constrained by the maximum thickness, chord, span and sweep values specified in the basic design specification. However, passenger accommodation, static stability, and structural considerations affect the final configuration. First, the flying and handling qualities required for this type of aircraft are outlined.

\subsubsection{Flying and Handling Qualities Requirement}

Under MIL-F-8785C (Cook (1997)) a passenger carrying LFW is a Class III aircraft, as it is large, heavy, and will demand low to medium manoeuvrability. The appropriate flight phases are categories B (climb, cruise and loiter) and C (takeoff, approach, overshoot and landing). Level 3 flying qualities are required, as the aircraft should be safely controllable, with Category B and C Flight Phases completable.

\subsubsection{Planform}

Planform geometry primarily affects the aircraft's stability characteristics, passenger-payload capacity, and vortex drag. With the latter dominated by span, which is already fixed, attention is restricted to the first two aspects.

\section{Centrebody Width}

The cabin/centrebody width is constrained by passenger comfort requirements. Consider the solution to a pure-rolling motion for a step aileron input (Nelson (1998))

$$
\frac{d \phi}{d t}=p_{s s}\left(1-e^{-t / \tau_{R}}\right)
$$

Equation 6.1 describes how bank angle varies with time for a given roll-mode time constant, where $p_{s s}$ is the steady-state roll rate. On integrating Eq. 6.1, with the initial boundary condition that $\phi=0$ at $t=0$,

$$
p_{s s}=\frac{\phi_{t g}}{t_{t g}-\tau_{R}\left(1-e^{-t_{t g} / \tau_{R}}\right)}
$$

Here we see that $p_{s s}$ depends partly on the time-constant $\left(\tau_{R}\right)$, and partly on the bank angle $\left(\phi_{t g}\right)$ and time $\left(t_{t g}\right)$ within which a manoeuvre should be executed - MIL-F-8785C specifies $30^{\circ}$ and $5 \mathrm{~s}$, respectively. 
The roll-induced acceleration $a$ is

$$
\begin{aligned}
a & =r \frac{d^{2} \phi}{d t^{2}} \\
& =r p_{s s} \frac{1}{\tau_{R}} e^{-t / \tau_{R}}
\end{aligned}
$$

where $r$ is the cabin half-width. The maximum roll acceleration (at $t=0$ ), $a_{\max }$, and outboard distance, $r$, from the roll axis are thus related via

$$
r=\frac{a_{\max }}{\left(\frac{d^{2} \phi}{d t^{2}}\right)_{t=0}}=a_{\max } \frac{\tau_{R}}{p_{s s}} .
$$

Figure 6.1 shows the variation of cabin half-width with the time constant $\tau_{R}$ for a range of maximum roll accelerations. Half-widths in the range of $5-30 \mathrm{~m}$ are expected over the range of vertical accelerations detailed. Furthermore, we see that, as $\tau_{R}$ becomes very large, the cabin half-width tends to a constant value. This is confirmed by considering the asymptotic solution to Eq. 6.2 and combining it with Eq. 6.4, showing that $r \rightarrow a_{\max } \frac{t_{t g}^{2}}{2 \phi_{t g}}$.

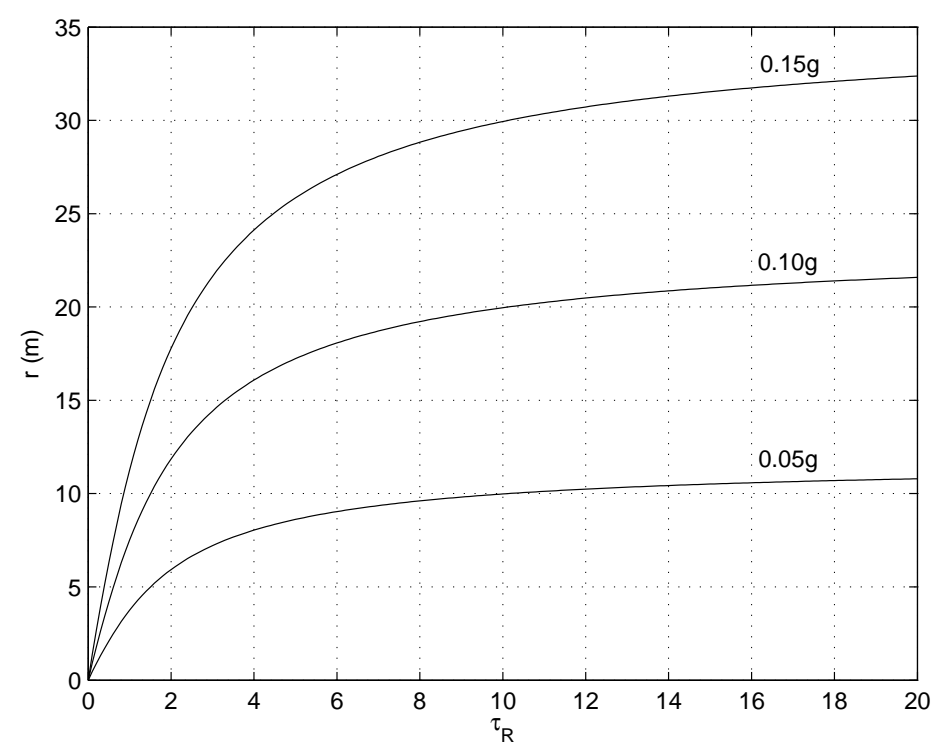

Figure 6.1: Variation of cabin half-width with roll-mode time constant for a range of vertical accelerations.

For a cabin half-width of $10 \mathrm{~m}$, roll-mode time constants in excess of $8 \mathrm{~s}$ are necessary to reduce the maximum vertical acceleration to a level recommended by Pratt (2000) of 


\section{CHAPTER 6. CONCEPTUAL DESIGN STUDY}

0.05-g, at the cost of compromised flying qualities. Assuming a 'flying plank' geometry with the allowable weight uniformly distributed gives a roll moment of inertia $\left(I_{x x}\right)$ of $59.8 \times 10^{6}$ kg.m ${ }^{2}$, which, combined with $C_{l_{p}}=-0.481 \mathrm{rad}^{-1}$ (see Sec. 6.1.2.3), provides an approximate estimate for $\tau_{R}$ of $0.62 \mathrm{~s}$ through Eq. 6.5 (provided by Nelson (1998)).

$$
\begin{aligned}
l_{p} & =C_{l_{p}} \frac{b}{2 U_{\infty}} \frac{Q S_{r e f} b}{I_{x x}} \\
\tau_{R} & =-\frac{1}{l_{p}}
\end{aligned}
$$

where $Q=1 / 2 \rho_{\infty} U_{\infty}^{2}$. This leads to a roll acceleration of 0.20 -g. Therefore, the roll-mode time constant must be artificially increased. As a compromise, a slightly higher than recommended vertical acceleration of $0.06-\mathrm{g}$ is accepted, with $\tau_{R}=4.5 \mathrm{~s}$.

To achieve an acceptable payload capacity the centrebody region is given a straight trailing edge. With the leading-edge sweep and cabin width now set, and chord length at the spanwise extremes specified by $t / c=0.20$, the centrebody planform geometry is fully defined.

\section{Stability Characteristics}

In Chap. 2 it was revealed that, provided the C.G. lies ahead of the neutral point (NP), satisfactory longitudinal stability characteristics can be achieved; furthermore, despite large differences in the lateral stability derivatives relative to conventional aircraft, flying wings still exhibit satisfactory lateral-directional stability characteristics. Therefore, work here is restricted to investigating how the planform parameters affect static stability. Zero wing-twist and symmetrical aerofoil sections are assumed.

The C.G. position is not yet known. Therefore, given the low sweepback and hence danger of the NP being ahead of the C.G., a neutral point that is as far aft as possible is targeted. For directional static stability, the lateral NP must also lie aft of the C.G. Furthermore, for lateral-directional stability, $C_{l_{\beta}}<0$ is a necessary condition.

To avoid adverse boundary-layer stability effects, the leading edge-sweep is fixed at $25^{\circ}$. With the centrebody region defined, two geometric parameters remain: outboard wing taper ratio and wingtip-fin height (assuming the fins are untapered and aligned perpendicular to the flow direction). Outboard wing taper ratios between 0.1 to 1 , and wingtip-fin heights of $2 \mathrm{~m}$ to $5 \mathrm{~m}$ are considered. Figure 6.2 provides an illustration of the planform geometry for extreme combinations of these two parameters - notice that for higher outboard wing taper ratios the centrebody becomes more distinguishable. 


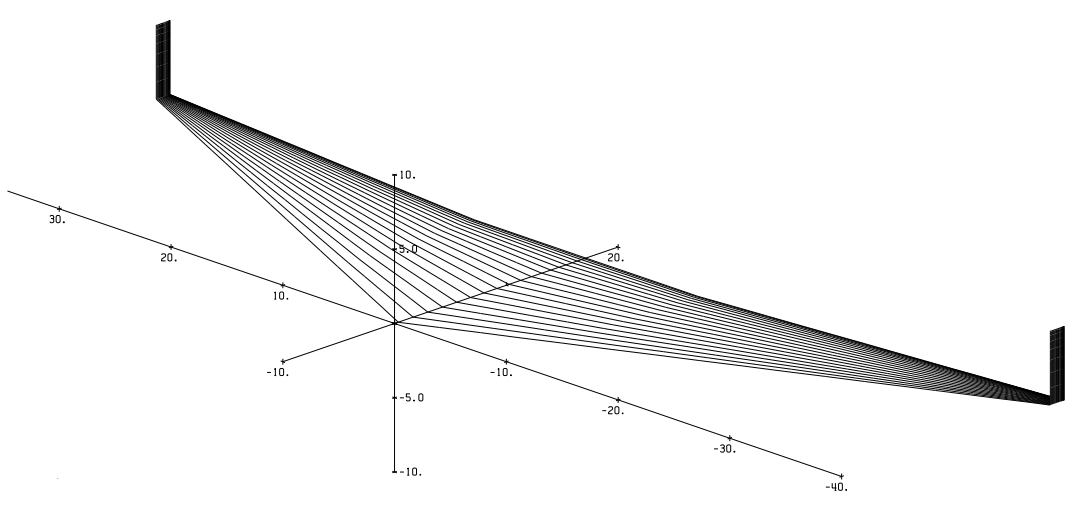

(a)

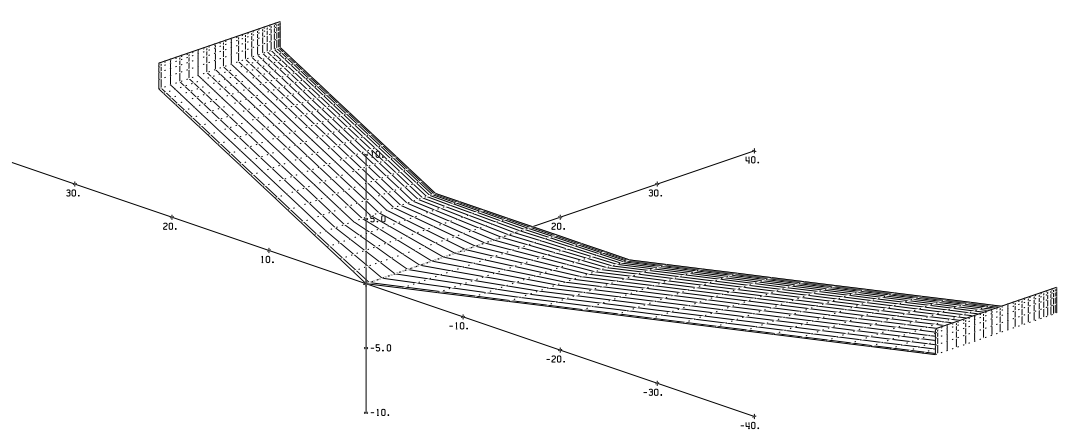

(b)

Figure 6.2: Schematic diagram of aircraft planform geometry: (a) $T_{R}=0.1$ and $H_{w}=5 \mathrm{~m}$ and (b) $T_{R}=1$ and $H_{w}=2 \mathrm{~m}$. 


\section{CHAPTER 6. CONCEPTUAL DESIGN STUDY}

\section{Longitudinal Neutral Point}

Figure 6.3 shows the variation in longitudinal NP position over a range of outboard wing taper ratios and wingtip-fin heights. The neutral point has a strong dependence on taper ratio, moving further aft as $T_{R}$ increases. For low taper ratios, wingtip-fin height has a weak effect, but becomes increasingly influential at higher taper ratios.

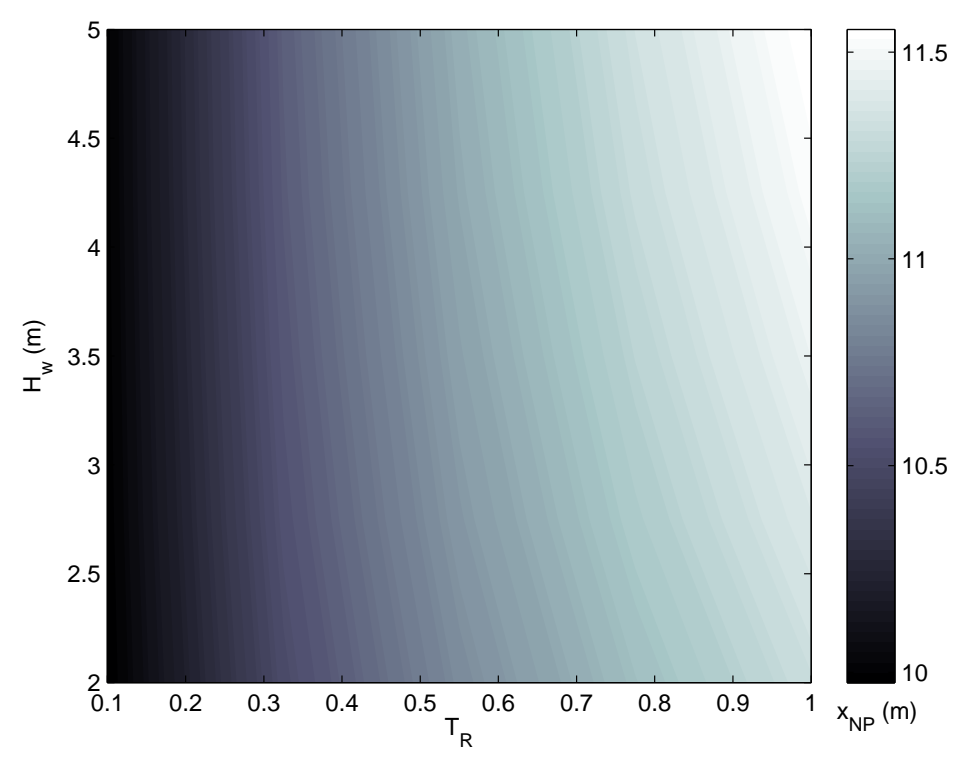

Figure 6.3: Variation in longitudinal NP position over a range of outboard wing taper ratios and wingtip-fin heights

For the geometric constraints imposed here, there is an increase in quarter-chord sweep angle associated with a high taper ratio, and hence the neutral point is further aft. At low taper ratios the effect of fin height is weak due to the reduced moment arm, despite the increase in tip loading with fin-tip height. To summarise, an aft longitudinal NP is favoured by a high taper ratio (higher quarter-chord sweepback) and large wingtip-fin height (increased tip loading).

\section{Lateral Neutral Point}

The lateral NP is defined as the location at which the sideforce due to sideslip effectively acts. Equation 6.6 shows that it depends on the weathercock and sideforce stability derivatives, 
and wing span

$$
x_{l a t, N P}-x_{r e f}=-b \frac{C_{n_{\beta}}}{C_{Y_{\beta}}},
$$

where $x_{r e f}$ is the point about which the stability derivatives are evaluated.

The effects of taper ratio and wingtip-fin height on the position of the lateral NP are shown in Fig. 6.4. The lateral NP moves further aft with increasing taper ratio due to the associated increase in sweepback; interestingly, this effect is strongest at low wingtip-fin heights. As for the longitudinal NP, wingtip-fin height effects become more apparent at higher taper ratios; however, somewhat counter-intuitively, the lateral NP moves further aft as wingtip-fin height decreases. For mid-range values of $T_{R}, X_{l a t, N P}$ travels forward then aftward with increasing $H_{w}$. Further examination of the dependence of the weathercock and sideforce stability derivatives on these parameters is required to understand these observations.

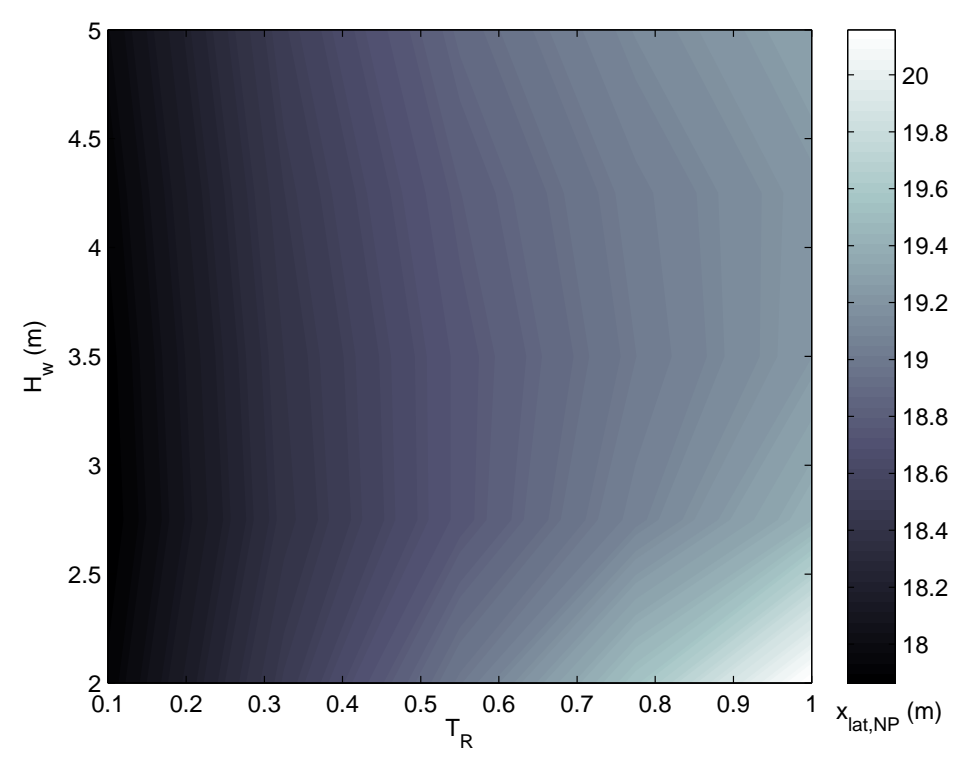

Figure 6.4: Variation in lateral NP position over a range of outboard wing taper ratios and wingtip-fin heights

Figure 6.5(a) shows how $C_{n_{\beta}}$ varies with $T_{R}$ and $H_{w}$. The yawing moment coefficient increases with wingtip-fin height due to the increased pressure force associated with a larger surface area. For a constant wingtip-fin height, $C_{n_{\beta}}$ initially increases with taper ratio due to the increased sweepback and chord length (and hence area) combination; however, this approaches a maximum for mid-range values of $T_{R}$ and subsequently, though rather mildly, drops with further increases in $T_{R}$ - this effect becomes weaker as $H_{w}$ is reduced. Figure 6.5(b) 


\section{CHAPTER 6. CONCEPTUAL DESIGN STUDY}

shows that the magnitude of the side-force stability derivative increases with tip-fin height, whilst it increases to a maximum and then drops with $T_{R}$ for a given $H_{w}$. Similar arguments for the effects on weathercock stability can also be applied to the side force. Therefore, it is because the sideforce stability derivative decreases at a faster rate than the weathercock stability derivative that, the lateral neutral point moves further aft with decreasing tip-fin height.

\section{Planform Summary}

With values for the longitudinal NP and lateral NP between $10-11.5 \mathrm{~m}$ and $18-20 \mathrm{~m}$, respectively, the longitudinal NP position is more critical. Furthermore, $C_{l_{\beta}}<0$ is satisfied for all combinations of taper ratio and fin height. Values for taper ratio and wingtip-fin height below the maximum considered are selected for structural efficiency, therefore: $T_{R}=0.9$ and $H_{w}=3.5 \mathrm{~m}$.

The final planform design is summarised in Fig. 6.8. The reference area and span are $1087.9 \mathrm{~m}^{2}$ and $80 \mathrm{~m}$, respectively. The aspect ratio is 5.9. The centrebody has a half-span of $10 \mathrm{~m}$ and a quarter-chord sweep of $18.9^{\circ}$. The outboard quarter-chord sweep angle is $24.5^{\circ}$.

The cruise and climb-out static margins, and longitudinal neutral points are detailed in Tab. 6.1. The aircraft exhibits close to neutral static stability characterisitcs over all flight phases of interest. Furthermore, the condition for spiral mode stability is $C_{l_{\beta}} C_{n_{r}} / C_{l_{r}} C_{n_{\beta}}>1$ : the LFW has values in the range $0.44-0.86$. A flight control system is therefore required to provide neutral spiral stability characteristics.

Table 6.1: Static stability - C.G. locations (see Sec. 6.1.5.5): $11.38 \mathrm{~m}$ (start of cruise and climb out) and $11.44 \mathrm{~m}$ (end of cruise).

\begin{tabular}{lccc}
\hline \hline Parameter & Cruise (with suction) & Cruise (no suction) & Climb out \\
\hline$M_{\infty}$ & 0.67 & 0.39 & 0.21 \\
$X_{N P}$ & 11.38 & 11.41 & 11.43 \\
Static margin (\%) & $0 /-0.5$ & $0.2 /-0.2$ & $0.4 /-0.1$ \\
$C_{l_{\beta}} C_{n_{r}} / C_{l_{r}} C_{n_{\beta}}$ & 0.86 & 0.60 & 0.44 \\
\hline \hline
\end{tabular}

\subsubsection{Wing and Cabin Design}

The planform layout, along with the need for structural efficiency, dictates the wing spar locations; this, combined with the need to maximise internal cabin volume, whilst observing the subcritical flow constraint, defines the wing cross-section across the aircraft centrebody. However, the outer wing regions are not subject to the same volumetric constraints. 


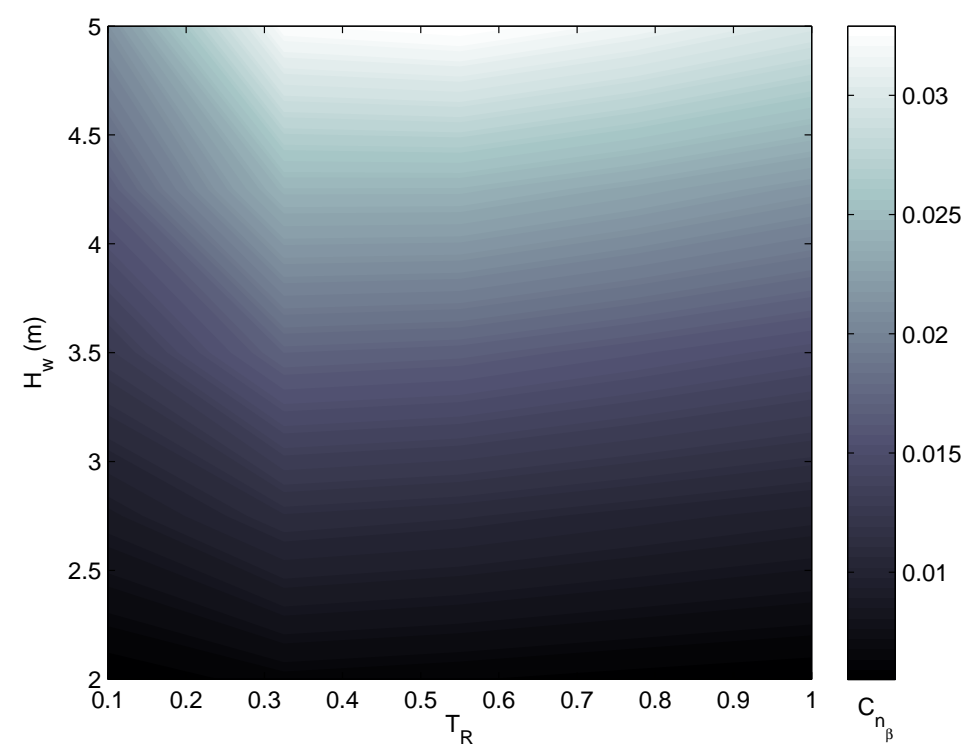

(a)

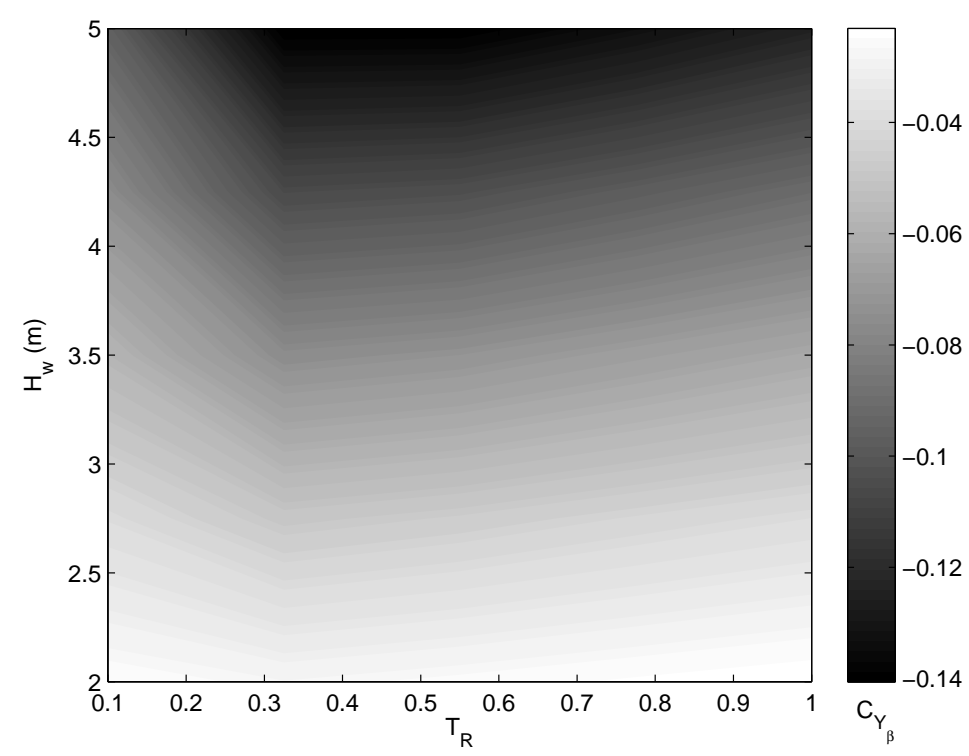

(b)

Figure 6.5: Change in (a) yawing moment coefficient and (b) side force with sideslip angle over a range of outboard wing taper ratios and wingtip-fin heights. 


\section{CHAPTER 6. CONCEPTUAL DESIGN STUDY}

The need for an aerofoil section exhibiting minimal nose-down pitching moment at zerolift was used, as an aerodynamic balance condition, to justify the selection of a NACA 4-digit section in Chap. 5. These are low-speed aerofoils, with a maximum thickness close to the leading edge (Abbott and Von Doenhoff (1959)). A more sophisticated approach, that maximises internal volume and improves transonic performance, is required here.

\section{Methodology}

Figure 6.6 shows a bespoke section generator (developed by Eastwood (2008)), which requires six variables to define an aerofoil section geometry. The section generator first takes the suction surface of the leading edge of a RAE2822 supercritical section, whose extent is marked by the first control point CP0, to ensure good performance in this aerodynamically critical region (Drela and Giles (1987)); the remaining surface is fitted by a cubic Bézier curve to the trailing edge, set by the control point CP3 whose vertical location lies along $x / c=1(y / c>0$ leads to a blunt trailing-edge section). To ensure continuity between the adjoining sections, the second control point CP1 is constrained to move along 'Track 1', which is tangential to the surface at the join. By defining a trailing-edge wedge angle $\theta_{t e}$, the third control point CP2 is constrained to move along 'Track 2', which is tangential to the surface at the trailing edge. The curve is then mirrored to form a symmetric section, and expanded to the required thickness.

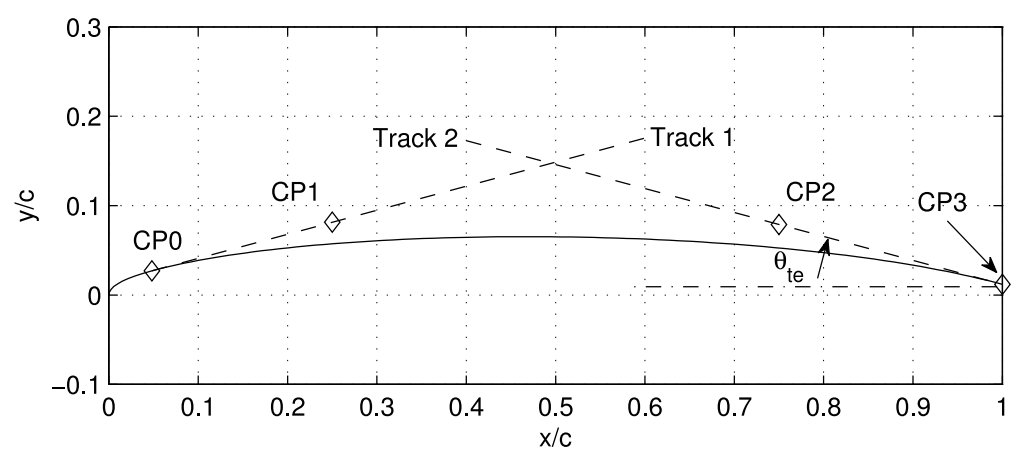

Figure 6.6: Aerofoil section generator.

To simplify the structural design task: a multi-bubble geometry comprising intersecting transverse cylinders is chosen for the pressurised cabin (see Sec. 6.1.5). The bubble overlap is chosen to: a) give a minimum height of $1.9 \mathrm{~m}$, based on the statistic, in Torenbeek (1976), that $99 \%$ of passenger heights are below this value, and b) have a seat pitch of $80 \mathrm{~cm}$ to 


\subsection{LAMINAR-FLYING-WING DESIGN CONCEPT}

$90 \mathrm{~cm}$, which Torenbeek (1976) cites as typical of conventional-type passenger carriers. A bubble diameter of $2.14 \mathrm{~m}$ is chosen, leading to a bubble pitch of $1 \mathrm{~m}$. Each seat row is assumed to lie on a bubble axis; consequently, the number of bubbles sets the number of rows. The number of seats abreast is determined by assuming one aisle every three seats; Torenbeek (1976) quotes values for seat and aisle widths of $42.5 \mathrm{~cm}$ and $50.8 \mathrm{~cm}$, respectively, which give an effective seat width of $59.4 \mathrm{~cm}$. The aerofoil section and number of bubbles are determined via trial and error:

a) multi-bubble arrangement arbitrarily defined between, and ahead, of the front and rear vertical spar positions (see Sec. 6.1.5);

b) aerofoil cross-section fitted to meet the various geometric constraints;

c) inviscid calculation using VGK in the perpendicular plane to ensure the flow is subcritical, and viscous calculation to ensure attached flow in cruise and takeoff; if not, iterate from b).

Figure 6.7 shows a cross-section of a representative multi-bubble cabin embedded within a centrebody wing section. Markers are placed at the front and rear bubble locations to denote minimum clearance requirements for the placement of suction hardware components and structural elements. At the spar locations, the bubbles intersect at the same height to prevent localised bending moments. To reduce surface curvature over the rear, a blunt trailing edge geometry is utilised. In contrast, sharp trailing-edge wing sections are employed outboard of the cabin.

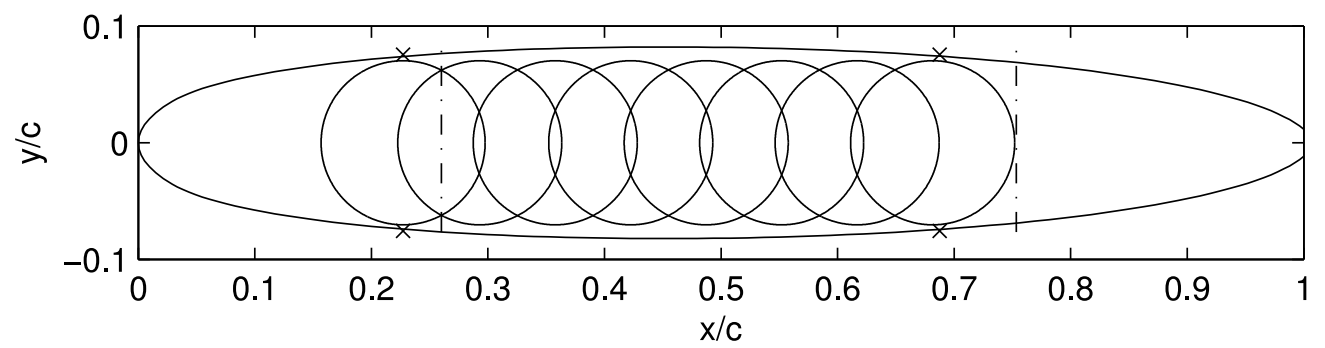

Figure 6.7: Schematic diagram of the multi-bubble cabin embedded within an aerofoil section - vertical spar locations are represented by the dash-dot lines, whilst the minimum spacings between the cabin and wing surface are indicated by the ' $x$ ' markers. 


\section{Specification}

Figure 6.8 details the final aerofoil and multi-bubble section geometries, and associated $C_{p}$ distributions calculated at the local section $C_{l}$ values (see Sec. 6.1.2). Specific details of the aerofoil sections are provided in Tab. 6.2.

Table 6.2: LFW section geometry variables specification.

\begin{tabular}{lccccc}
\hline \hline Parameter & $\mathrm{y}=0.84 \mathrm{~m}$ & $\mathrm{y}=4.20 \mathrm{~m}$ & $\mathrm{y}=9.18 \mathrm{~m}$ & $\mathrm{y}=10-40 \mathrm{~m}$ & $\mathrm{z}=0-3.5 \mathrm{~m}$ \\
\hline$(t / c)_{\max }$ & 0.149 & 0.164 & 0.194 & 0.20 & 0.10 \\
$C P 0 X$ & 0.03 & 0.03 & 0.08 & 0.10 & 0.10 \\
$C P 1 X$ & 0.15 & 0.15 & 0.26 & 0.40 & 0.40 \\
$C P 2 X$ & 0.91 & 0.90 & 0.80 & 0.85 & 0.85 \\
$C P 3 Y$ & 0.012 & 0.008 & 0.002 & 0 & 0 \\
$\theta_{\text {te }}(\mathrm{deg})$ & 28 & 26 & 17 & 5 & 5 \\
\hline \hline
\end{tabular}

Along the central axis, the reduced maximum thickness-to-chord ratio permits the placement of bubbles ahead of the front spar, whilst a blunt trailing edge is required to alleviate flow conditions over the rear. Moving out across the centrebody, the (non-dimensional) rear spar location effectively moves forwards as the chord drops. This permits a lower trailingedge wedge angle and thickness. With the leading edge swept, the distance between the front spar and nose drops, therefore the number of bubbles ahead of the front spar decreases. To allow for a smooth transition to the outboard region, the outer centrebody section is scaled to the required $t / c$, and a sharp trailing-edge is enforced. The fin sections are scaled down versions of the outer sections with $t / c=0.10$.

The total cabin floor area is around $138 \mathrm{~m}^{2}$, but approximately $7 \mathrm{~m}^{2}$ is required for items such as: wardrobes, toilets, etc., for a 200-passenger aircraft (Torenbeek (1976)). Therefore, the passenger specification for the LFW is 220 persons. 


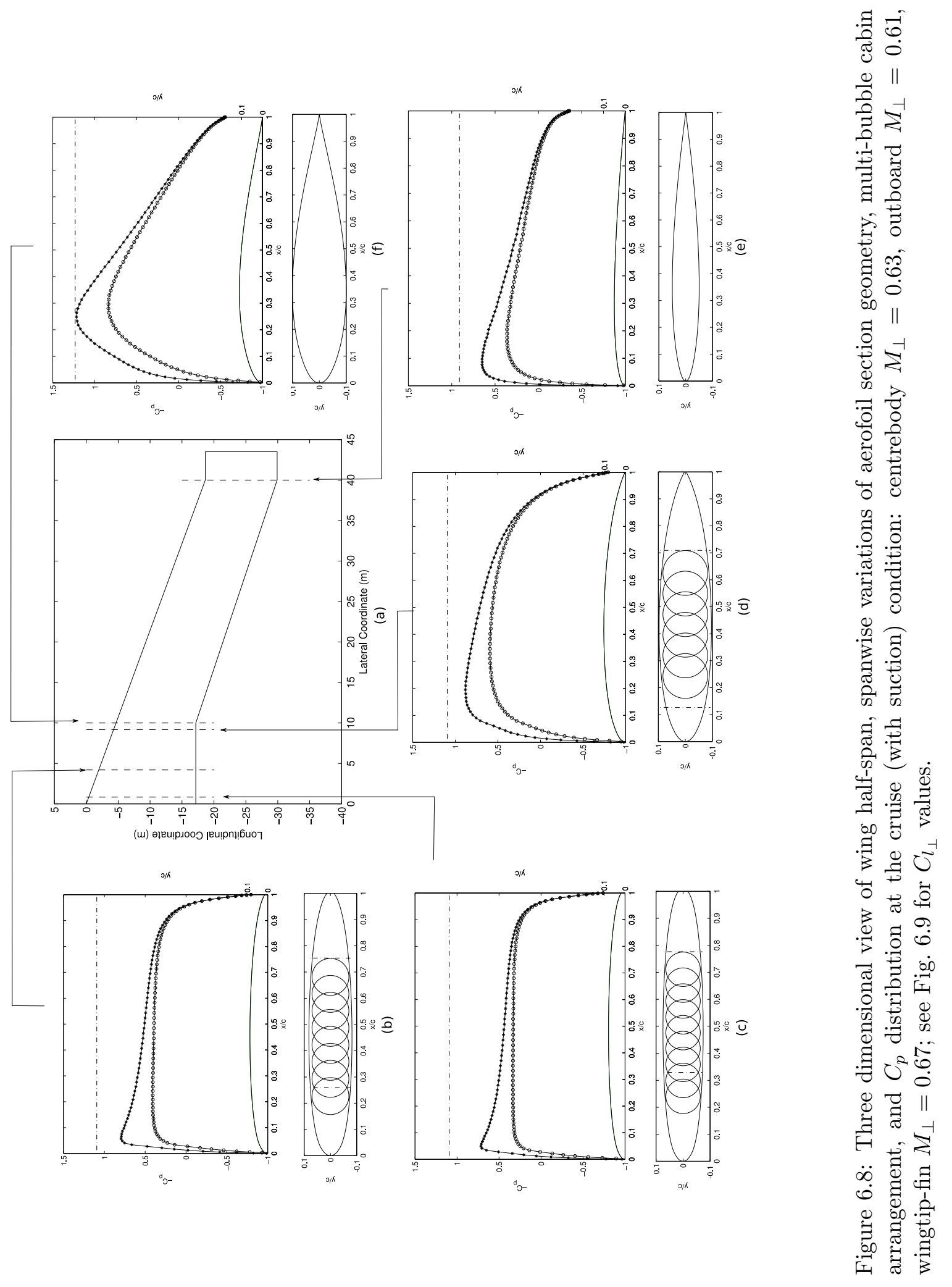




\section{CHAPTER 6. CONCEPTUAL DESIGN STUDY}

\subsubsection{Aircraft Performance}

The aircraft performance is already broadly defined by the cruise $C_{L}$, wing loading, cruise Mach number and unit Reynolds number. However, in this section, it is further broken down into cruise and low-speed performance. In Fig. 3.1, aircraft performance is shown to be central to the overall design integration. For example, the cruise lift distribution affects the suction-system design and the wing section geometry; furthermore, the loading distribution is necessary for a structures and weights analysis, which in turn provides the C.G. position. The minimisation of non-LFC surface areas constrains control surface sizes; however, satisfactory control characteristics must be ensured at low-speed conditions. The lift-to-drag ratio for both flight conditions affects engine performance and is essential for evaluating aircraft range. Before proceeding with an assessment of cruise and low-speed performance, an exposition of the methods used to assess pitch trim and calculate viscous drag is provided.

\subsubsection{Methodology}

\section{Pitch Trim}

In contrast to traditional definitions given in Chap. 2, the whole elevon is called the elevator, whilst the outer elevons are referred to as ailerons. The elevator is assumed to occupy $67 \%$ of the wing half-span (starting from the wingtip fin) and the last $10 \%$ of chord. The associated pitching moment has to balance contributions (about the centre of gravity) from the engine thrust and the wing in flight; whilst on the ground there are additional reaction and friction forces acting through the main undercarriage (assumed to be located at $75 \%$ chord at the outboard extremes of the aircraft centrebody). (The thrust line is taken to be $3.325 \mathrm{~m}$ above the aerofoil's axis of symmetry, which corresponds to half an engine diameter (see Sec. 6.1.4) above the point of maximum aerofoil section height.)

The required elevator deflection and aircraft angle of attack are found through the solution of Eq. 6.7.

$$
\left[\begin{array}{c}
C_{L_{\text {target }}} \\
C_{m_{\text {target }}}-C_{m_{0}}
\end{array}\right]=\left[\begin{array}{cc}
C_{L_{\alpha}} & C_{L_{\delta_{E}}} \\
C_{m_{\alpha}} & C_{m_{\delta_{E}}}
\end{array}\right]\left[\begin{array}{c}
\alpha \\
\delta_{E}
\end{array}\right]
$$

With symmetrical aerofoil sections and zero twist, $C_{m 0}=0 ; C_{L_{\text {target }}}$ and $C_{m_{\text {target }}}$ correspond to the flight phase of interest. These, in addition to the stability derivatives, are summarised in Tab. 6.4. 


\subsection{LAMINAR-FLYING-WING DESIGN CONCEPT}

\section{Viscous Drag}

The wing viscous drag for a swept wing is calculated following the method detailed in Chap. 3. However, the Squire-Young formulation of Eq. 3.5 is not applicable to blunt-trailing-edge aerofoils. Therefore, conservatively, the above-ambient trailing-edge pressure is neglected, and the momentum deficit at the trailing edge used directly, i.e.,

$$
C_{D_{v}}=2 \frac{\delta_{2, t e}}{c} .
$$

For small trailing-edge thicknesses, Eq. 6.8 gives a conservative estimate of the viscous drag, as it fails to account for pressure recovery effects in the wake. The overall wing viscousdrag coefficient $C_{D_{v}}^{\text {wing }}$ is estimated by averaging the section-viscous drag coefficients over the planform area. For the low section $C_{l}$ s here, it is assumed that the zero-lift viscous drag $C_{D 0}^{\text {wing }} \simeq C_{D_{v}}^{\text {wing }}$. Additional viscous drag contributions are determined following the methods detailed in Chap. 3.

\subsubsection{Cruise Performance}

The combination of $C_{m 0}=0$, near-neutral static stability, and low thrust requirement in cruise (with suction) translates to a negligible elevator deflection of $-0.1^{\circ}$ for aircraft trim. The wing incidence $\alpha$ is $1.5^{\circ}$ - this is below a value of $3^{\circ}$, which Hileman et al. (2007) provide as a recommended fuselage maximum.

The lift coefficient distribution is shown in Fig. 6.9. With a linear decrease in chord across the centrebody, section $C_{l_{\perp}}$ increases from around 0.15 to a maximum of 0.20 , but then decreases to 0.10 at the wingtip fins. The streamwise and section-normal curves coincide at $y>40 \mathrm{~m}$ as the wingtip fin is unswept. The calculated Oswald efficiency $e$ is 1.08. This favourable value is attained thanks to the efficient all-lifting wing surface and wingtip-fin combination, and is not compromised by the trim requirements here.

A drag breakdown is provided in Tab. 6.3. Figure 6.10 details the variation in wing viscous drag coefficient (based on local chord length) across the inboard and outboard regions. The sharp reduction in viscous drag at the centrebody/outboard junction and increase between $y=4.2-5.8 \mathrm{~m}$ are associated with changes in trailing-edge thickness. With a negligible influence of $C_{l}$ at these low values, it is the reduction in chord Reynolds number that contributes to an overall increase across the centrebody; whilst the negligible change in $R e_{c}$ outboard leads to an almost constant coefficient.

The calculated lift-to-drag ratio is 60.9. This figure is significantly higher than current, turbulent, jet aircraft with values of $15-20$ (cited by Cumpsty (1997)). 


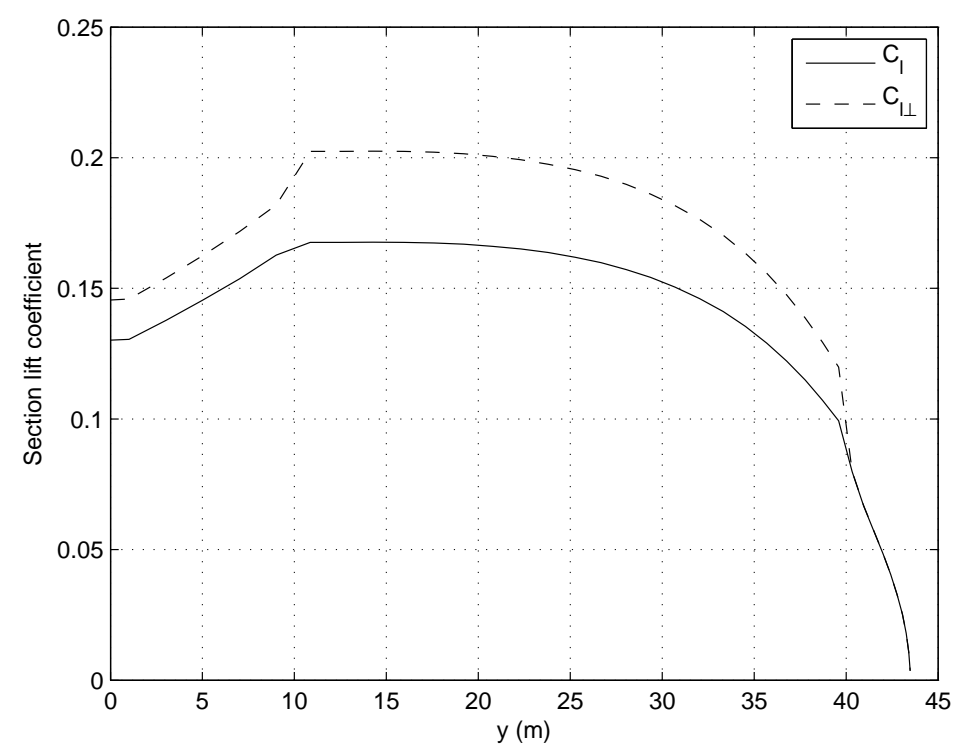

Figure 6.9: Spanwise variation in section lift coefficient in the free-stream and wing normal directions $-C_{L}=0.14$ and $M_{\infty}=0.67$.

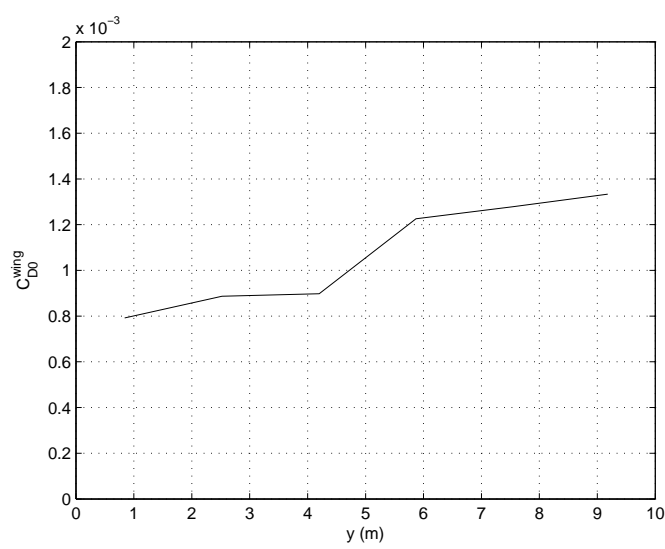

(a) Inboard

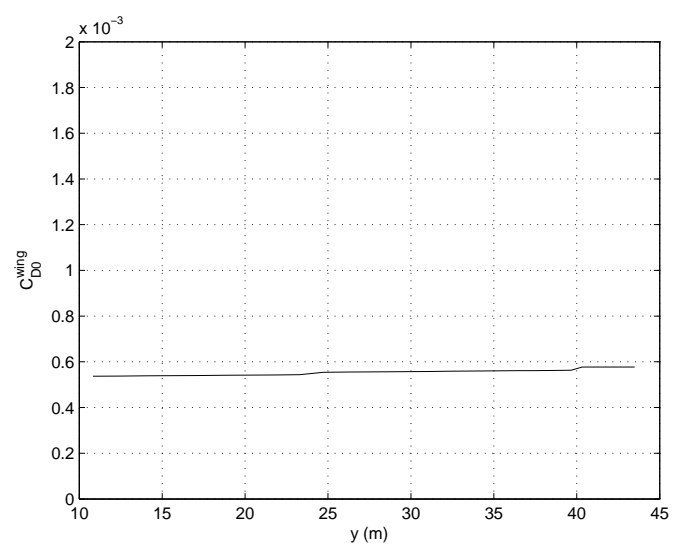

(b) Outboard

Figure 6.10: Variation in viscous drag coefficient along the wing half-span $-R e_{u}=8 \times 10^{6}$ $\mathrm{m}^{-1}$

In the event of a suction-system failure, the loss of laminar flow results in a significant increase in total viscous drag. Continuing to fly at a lift coefficient optimised for minimum fuel consumption with suction on is far from optimum, as $C_{L} \propto \sqrt{C_{D 0}}$ (see Chap. 5). The 
revised cruise $C_{L}$ and flight Mach number are 0.38 and 0.39 , respectively, assuming constant cruise altitude. With a higher thrust requirement, an elevator deflection of $-0.8^{\circ}$ is required to trim the aircraft, whilst the wing incidence increases to $4.8^{\circ}$. The Oswald efficiency remains unchanged at around 1.08. The lift-to-drag ratio is significantly degraded at 24.4 (see Tab. 6.3).

\subsubsection{Low-Speed Performance}

\section{Takeoff $C_{L}$}

The stalling speed and corresponding maximum lift coefficient for a B737-200 aircraft are 63.7 $\mathrm{m} / \mathrm{s}$ and 2.07, respectively (see Chap. 5). Assuming a takeoff speed of $70 \mathrm{~m} / \mathrm{s}$, the takeoff $C_{L}$ and Mach number are 0.61 and 0.21 , respectively. Therefore, $C_{L, \max }$ is unlikely to constrain the design here.

\section{Pitch Trim and Elevator Performance}

During climb out, the elevators are required to trim the aerodynamic ( $L$ and $M_{C . G .}$ ) and thrust $\left(F_{N}\right)$ forces acting on the aircraft by generating a force $\left(L_{E}\right)$, at the demanded takeoff $C_{L}$. However, for rotation at takeoff, with the nose wheel off the ground, the reaction force $(R)$ due to the weight of the aircraft and associated friction force $\left(F_{\mu}\right)$ - Raymer $(1999)$ gives a typical rolling friction coefficient of 0.03 - through the main undercarriage, contribute to an additional nose down moment, as detailed schematically in Fig. 6.11.

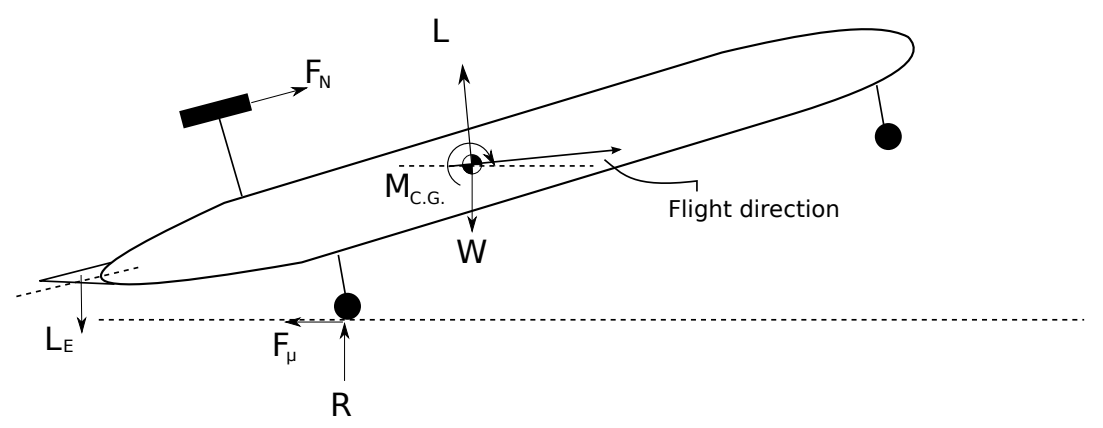

Figure 6.11: Force diagram at takeoff rotation.

The speed of the aircraft at rotation is estimated to be approximately $66.8 \mathrm{~m} / \mathrm{s}$, following Torenbeek (1976). With all engines operating, the demanded elevator deflection for rotation at takeoff is found to be more critical, with a $\delta_{E}$ of $-20.2^{\circ}$ and an $\alpha$ of $11.3^{\circ}$. (Note, Raymer 


\section{CHAPTER 6. CONCEPTUAL DESIGN STUDY}

(1999) comments that ground effect typically increases the lift-curve slope by up to $10 \%$ for conventional aircraft; however, this has been neglected here.)

With one engine out on climb-out, an elevator deflection $\delta_{E}$ of $-2.5^{\circ}$ and wing incidence $\alpha$ of $8.3^{\circ}$ are required to trim the aircraft. The upwards elevator deflection has a beneficial influence on Oswald efficiency which increases to 1.09 from 1.08 (corresponding to zero elevator deflection). Note, the lift-to-drag ratio corresponding to this flight condition is used to evaluate the required engine thrust in Sec. 6.1.4: it has a value of 22.4.

\section{Rudder Performance}

Rudders are located on the trailing edge of the wingtip fins for directional control. The rudders are sized to provide sufficient yaw authority at engine-out upon takeoff for maximum available thrust (see Sec. 6.1.4). At the critical C.G location $\left(x_{C . G .}=11.38 \mathrm{~m}-\right.$ see Sec. 6.1.5.5), $\frac{\partial C_{n}}{\partial \delta_{r}}=0.00688$. With a single engine located along the central axis, an offset of $7 \mathrm{~m}$ for the remaining two engines yields a $C_{n}$ of 0.00174 . For rudders that occupy the last $10 \%$ of chord and extend across the full height of the wingtip fin, an asymmetric deflection of $\delta_{r}=14.5^{\circ}$ is demanded of both rudders. A viscous XFoil calculation, with a chord Reynolds number of 53.8 million and required maximum section $C_{l}$ of around 0.5 , reveals that the flow remains attached.

\section{Aileron Performance}

The classical steady-state solution to the equation of motion for a pure rolling motion, as given by Nelson (1998), is

$$
\frac{p_{s s} b}{2 U_{\infty}}=-\frac{C_{l_{\delta_{a}}}}{C_{l_{p}}} \Delta \delta_{a}
$$

The change in $C_{l_{\delta_{a}}} / C_{l_{p}}$ over the various flight phases is found to be negligible in comparison to the change in flight speed. Therefore, maximum aileron deflection is expected to occur during a banked motion on climb out.

For ailerons that occupy the outer $50 \%$ of the the elevon's span, the roll-moment coefficient due to aileron deflection $C_{l_{\delta_{a}}}$ is $0.114 \mathrm{rad}^{-1}$, whilst the roll-damping moment coefficient $C_{l_{p}}$ is $-0.481 \mathrm{rad}^{-1}$. A minimum $30^{\circ}$ bank angle change within 5 seconds and $\tau_{R}=4.5$ s, i.e., $p_{s s}=0.149 \mathrm{rad} / \mathrm{s}$ (see Sec. 6.1.1), is achieved with an average deflection $\delta_{a}$ of $12.2^{\circ}$. At takeoff, the trim requirement in pitch means that there is already a $-2.5^{\circ}$ elevator deflection; therefore, the required left and right aileron deflections for a clockwise roll would be $9.7^{\circ}$ and $-14.7^{\circ}$, respectively. Figure 6.12 shows that the critical values for section $C_{l}$ over these surfaces are 0.83 and 0.2 , respectively. A viscous XFoil calculation with $R e_{c}=60 \times 10^{6}$ 
reveals that the flow remains attached for both cases.

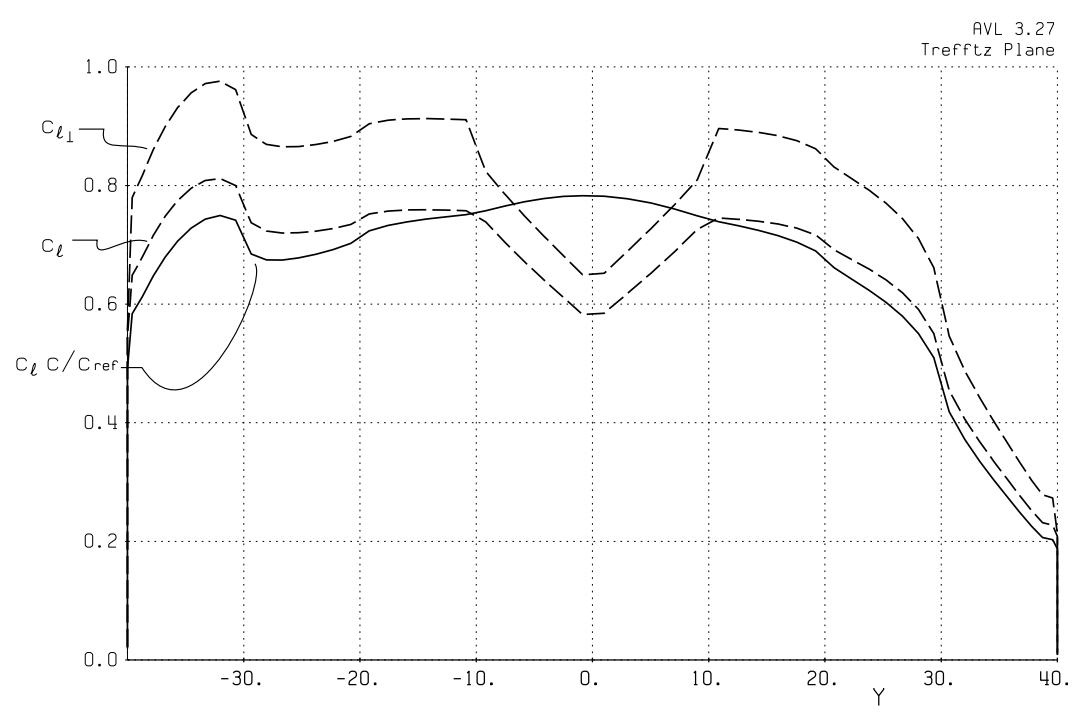

Figure 6.12: Spanwise lift distribution corresponding to a clockwise roll at take off with: $\delta_{a}=+/-12.2^{\circ}$ and $\delta_{E}=-2.5^{\circ}: M_{\infty}=0.21$ and $C_{L}=0.61$.

Table 6.3: LFW lift and drag coefficient breakdown.

\begin{tabular}{lccc}
\hline \hline Coefficient & Cruise (suction) & Cruise (no suction) & Climb out \\
\hline$C_{L}$ & 0.14 & 0.38 & 0.61 \\
$C_{D}$ & 0.0023 & 0.01535 & 0.02721 \\
$C_{D_{i}}$ & 0.00098 & 0.0072 & 0.01838 \\
$C_{D_{v, \text { wing }}}$ & 0.00067 & 0.0075 & 0.0080 \\
$C_{D_{v, \text { misc }}}$ & 0.00065 & 0.00065 & 0.00083 \\
$L / D$ & 60.9 & 24.8 & 22.4 \\
\hline \hline
\end{tabular}

\subsubsection{Suction-System Technology Integration}

In Fig. 3.1 we saw that suction-system technology integration is influenced by the airframe, and aircraft performance. The extent of laminarised wetted area is limited by the need for satisfactory control authority at low speeds. The wing cross section not only influences the extent of laminarisation through geometric constraints at the trailing edge but, when combined with the lift distribution in cruise (for a given planform, cruise $C_{L}$ and Mach number), determines the surface pressure distribution, which in turn sets the level of distributed suction, and hence suction-pump power. The suction system in turn affects cruise performance, 
Table 6.4: LFW aerodynamic performance parameters for climb-out and takeoff with one engine inoperative, and cruise, with C.G. at $11.38 \mathrm{~m}$.

\begin{tabular}{lccc}
\hline \hline Derivative & Cruise (suction) & Cruise (no suction) & Climb out / Takeoff rotation \\
\hline$C_{L}$ & 0.14 & 0.38 & 0.61 \\
$M_{\infty}$ & 0.67 & 0.39 & 0.21 \\
$C_{L_{\alpha}}\left(\mathrm{rad}^{-1}\right)$ & 5.239 & 4.629 & 4.417 \\
$C_{L_{\delta_{E}}}\left(\mathrm{rad}^{-1}\right)$ & 0.834 & 0.762 & 0.742 \\
$C_{m_{\alpha}}\left(\mathrm{rad}^{-1}\right)$ & 0.00115 & -0.0100 & -0.0163 \\
$C_{m_{\delta_{E}}}\left(\mathrm{rad}^{-1}\right)$ & -0.490 & -0.433 & -0.424 \\
$C_{m, t a r g e t}$ & 0.00056 & 0.0049 & $0.0161 / 0.146$ \\
$\alpha(\mathrm{deg})$ & 1.5 & 4.8 & $8.3 / 11.3$ \\
$\delta_{E}(\mathrm{deg})$ & -0.1 & -0.8 & $-2.5 /-20.2$ \\
\hline \hline
\end{tabular}

whilst extraction of the required suction power affects engine performance, and the associated hardware weight affects the structure and its weight.

In arriving at the aircraft design specification in Chap. 5, the optimum suction distribution for maintenance of laminar flow was combined with the aerofoil surface pressure distribution to obtain the optimum pump and viscous drag. However, for a detailed conceptual design study it is necessary to include the effects of a practical system architecture. The approach here is to take the basic arrangement proposed in Chap. 4 and apply it to the aircraft configuration shown in Fig. 6.8.

\subsubsection{System Architecture}

An illustration of the hardware arrangement for the centrebody region is detailed in Fig. 6.13. Ideally, the spanwise chambers should be continuous across the span. This is made difficult by large variations in surface $C_{p}$ associated with an increasing thickness-to-chord ratio (see Fig. 6.8), which would lead to violation of the incompressible flow assumption described in Chap. 4. By tailoring the chamber sets and corresponding pressures to each surface pressure distribution, high differential pressures are avoided. Therefore, the spanwise chamber arrangement is divided across three regions (with the dash-dot lines corresponding to changes in wing-section geometry). Flow rate controllers throttle the flow between chambers, which is then ducted into a set of chordwise ducts (each attached to a suction pump) located along the centrebody/outboard junction, and ejected at flight speed along the trailing edge of the centrebody. The grey areas correspond to regions where suction is not applied: over the leading edge suction is not required until some distance aft (see Chap. 4), whilst hardware space requirements and the presence of trailing-edge control surfaces limit its extent to the 
first $90 \%$ of chord.

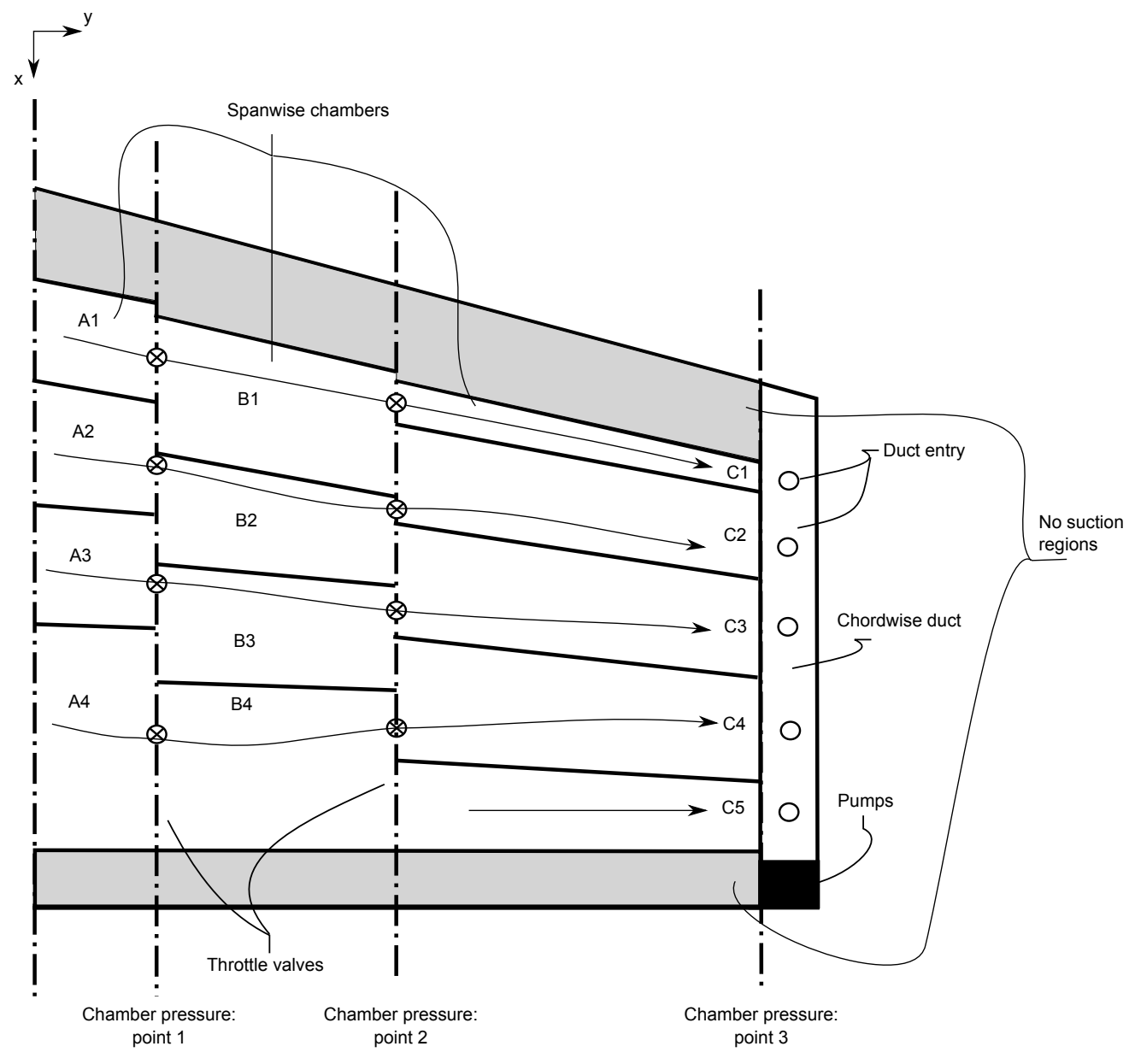

Figure 6.13: Typical inboard suction-system architecture.

With no variation in wing section across the outboard and wingtip-fin regions, the arrangement consists of (separate) continuous chamber sets over each region. However, the sucked flow from the wingtip fins is throttled to match the chamber pressures of the outboard wings. The flow is subsequently transported to the collector ducts and pumps at the centrebody/outboard junction.

\subsubsection{System Specification}

The system architecture over each region is specified via the algorithm provided in Chap. 4 . However, ensuring that each region has a similar number of spanwise chambers introduces a further constraint on the ratio of non-dimensional pressure difference across the skin over 


\section{CHAPTER 6. CONCEPTUAL DESIGN STUDY}

a single chamber width $\left(\widetilde{r}_{c}\right)$. With reference to Fig. 6.13 , an outline of the LFW system architecture specification algorithm is given below:

a) a boundary layer calculation at the inboard extreme of a region, where $R e_{c}$ is highest (leading to earlier transition prediction and suction initiation), sets the chamber layout and pressure, surface porosity distribution, and hence suction velocity distribution;

b) as $R e_{c}$ reduces outboards, for a constant distribution of skin porosity and chamber pressure, the associated level of distributed suction is insufficient to maintain laminar flow; therefore, the skin porosity coefficient, $K_{s}$, is increased uniformly across the region of interest until the suction level is sufficient at the outboard extreme of a region;

b) chamber depths are set such that inertial and frictional effects are small relative to the differential pressure across the skin, using Eq. 4.17;

c) the chamber pressures in region $\mathrm{B}$ are set higher than those in $\mathrm{C}$, and those in $\mathrm{A}$ higher than in $\mathrm{B}$, to attain the desired flow direction;

d) the 'real' suction distribution calculated over each region is combined with the chamber pressure distribution over region $\mathrm{C}$, which sets the pump inlet pressures, to provide an estimate of the suction power.

For low values of $C_{l}$, it was shown in Chap. 4 that this parameter has a negligible effect on the suction requirements; therefore, spanwise variations in $C_{l}$ across a region are neglected. However, the highest values of $C_{l}$, shown in Fig. 6.9, over a spanwise region, are used to evaluate the suction requirements: these correspond to the surface $C_{p}$ distributions shown in Fig. 6.8.

Table 6.5 summarises the chamber properties for the inboard region. The maximum number of chambers on the upper and lower surfaces is five and four, respectively; the minimum being two and four, respectively. Fewer chambers are required on the lower surface given the reasonably flat surface $C_{p}$ distribution (see Fig. 6.8).

Table 6.5: Chamber specifications for the inboard section.

\begin{tabular}{lccc}
\hline \hline Parameter & $y<=0.84 \mathrm{~m}$ & $y<=4.20 \mathrm{~m}$ & $y<=10 \mathrm{~m}$ \\
\hline$\Delta \widetilde{P}_{s, a v}$ & 0.15 & 0.15 & 0.15 \\
$\widetilde{r}_{c}$ & 4 & 4 & 6 \\
$k=K_{s, \text { actual }} / K_{s, \text { ideal }}$ & 1.1 & 1.1 & 1.1 \\
No. Chambers (upper / lower) & $4 / 2$ & $4 / 2$ & $5 / 4$ \\
\hline \hline
\end{tabular}




\subsection{LAMINAR-FLYING-WING DESIGN CONCEPT}

The distributions of surface pressure and chamber pressure (provided spanwise chamber losses are small) are detailed in Fig. 6.14 for region $\mathrm{B}(0.84<y<=4.20 \mathrm{~m})$ and region $\mathrm{C}$ $(4.20<y<=9.18 \mathrm{~m})$. The solid line is the chamber pressure distribution across region B, and is determined based on consideration of the surface pressure distribution, indicated by the dash-dot line. Region B has one small chamber upstream of the suction peak, and three downstream. The chamber pressures in region $\mathrm{C}$ are lower than those over region $\mathrm{B}$, and hence ensure the desired flow direction. (Note, that the last chamber of region $\mathrm{C}$ is isolated and not connected to region B.)

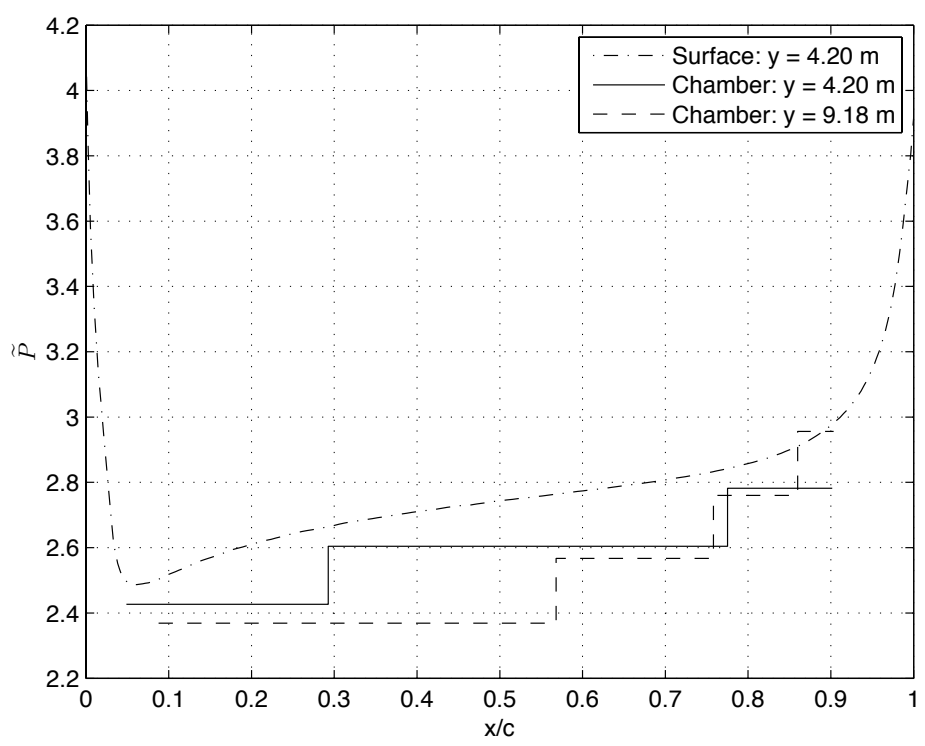

Figure 6.14: Distribution of inboard chamber and surface (non-dimensional) pressures across regions $\mathrm{B}$ and $\mathrm{C}$.

The critical differential pressures, corresponding to a flow Mach number of 0.4 across the porous skin, for the three regions considered are detailed in Fig. 6.15. The maximum $\Delta \widetilde{P}_{s}$ across the skin is below critical, and therefore the assumption of incompressible flow is satisfactory. A maximum chamber depth of around $3 \mathrm{~cm}$ is required to keep chamber pressure losses small.

The chamber specifications for the outboard and wingtip fin regions are summarised in Tab. 6.6. The sharp trailing-edge constraint results in a steep pressure gradient over the rear of the outboard aerofoil, leading to a greater number of spanwise chambers relative to the inboard regions. However, relative to the outboard region, fewer chambers are required over 


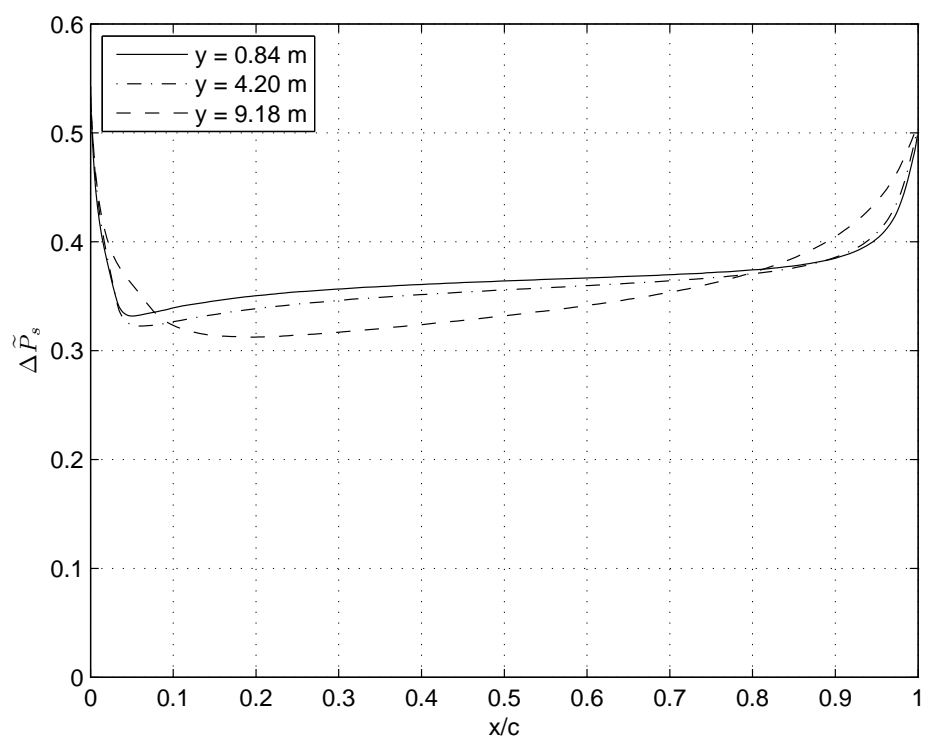

Figure 6.15: Critical (non-dimensional) differential pressures across porous skin along the centrebody region.

the wingtip fins because of the reduction in maximum thickness-to-chord ratio. As with the inboard region, the flow across the porous skin may be treated as incompressible.

Table 6.6: Chamber specifications for the outboard section.

\begin{tabular}{lcc}
\hline \hline Parameter & $y<=40 \mathrm{~m}$ & $z<=3.5 \mathrm{~m}$ \\
\hline$\Delta \widetilde{P}_{s, a v}$ & 0.15 & 0.15 \\
$\widetilde{r}_{c}$ & 6 & 3 \\
$k=K_{s, \text { actual }} / K_{s, \text { ideal }}$ & 1.1 & 1 \\
No. Chambers (upper / lower) & $8 / 6$ & $6 / 4$ \\
\hline \hline
\end{tabular}

However, with a span of $33 \mathrm{~m}$, the outboard spanwise chambers are prone to high chamber pressure losses. Ignoring effects of wing taper and additional suction flow entering from the wingtip fins, Fig. 6.16 details the pressure drop for three chamber depths: $0.05 \mathrm{~m}, 0.10 \mathrm{~m}$ and $0.15 \mathrm{~m}$, determined using Eq. 4.17. It reveals that chamber depths greater than $0.10 \mathrm{~m}$ are necessary to ensure that chamber pressure losses remain small relative to the pressure drop across the skin. 


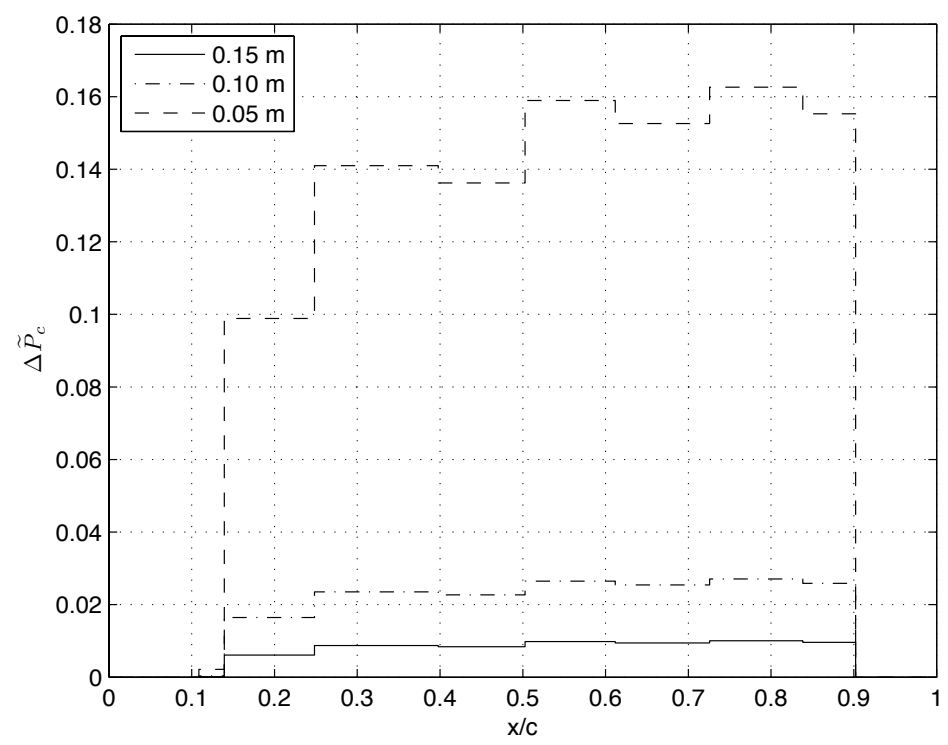

Figure 6.16: Outboard (non-dimensional) chamber pressure drop.

\subsubsection{Power Requirement and Weight Penalty}

Figure 6.17 details the spanwise variation in suction coefficient $C_{Q}$ and pump power coefficient $\widetilde{W}_{p}$. The (dimensionless) suction requirements increase across the centrebody due to the increase in section thickness-to-chord ratio and reduction in chord Reynolds number. A reduction in trailing-edge thickness and increase in $t / c$ lead to a sharp increase between $y=$ $4.2-5.8 \mathrm{~m}$ and at the centrebody/outboard intersection. Wingtip-fin suction requirements are lower, given the reduced $t / c$.

The overall $C_{Q}$ is $3.10 \times 10^{-4}$ and, assuming a suction pump efficiency $\eta_{p}$ of $85 \%, \widetilde{W}_{p}$ is $6.3 \times 10^{-4}$. With a planform area of $1088 \mathrm{~m}^{2}, U_{\infty}=209 \mathrm{~m} / \mathrm{s}$ and $\rho_{\infty}=0.599$ : the total suction mass flow is $43.5 \mathrm{~kg} / \mathrm{s}$ and the power to energise the suction system is $1.87 \mathrm{MW}$. The suction pump weight is estimated to be $895 \mathrm{~kg}$, assuming a pump weight of $0.48 \mathrm{~kg}$ per $\mathrm{kW}$ (estimated by Wilson (1997)). The spanwise chambers are integrated within the aircraft structure, and are therefore not included in the weight estimate here - see Sec. 6.1.5.

\subsubsection{Propulsion System}

Recall, Fig. 3.1 shows that the propulsion units are required to power the suction system, generate enough thrust at takeoff, and have a satisfactory level of fuel consumption. Also, 


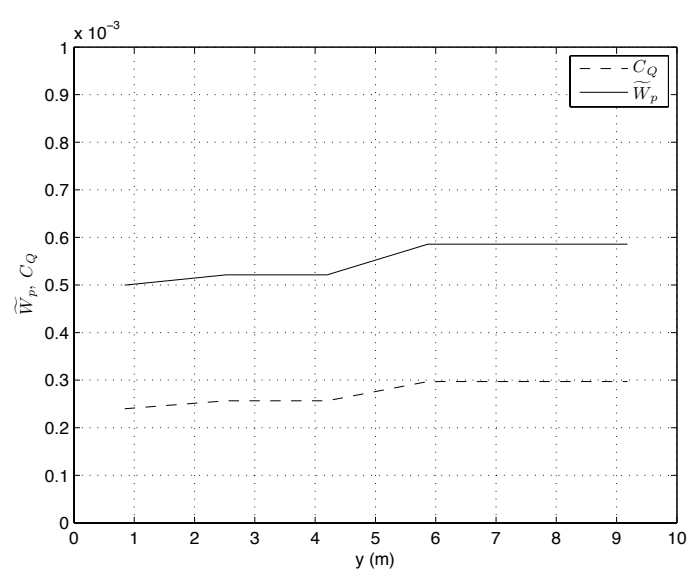

(a) Inboard

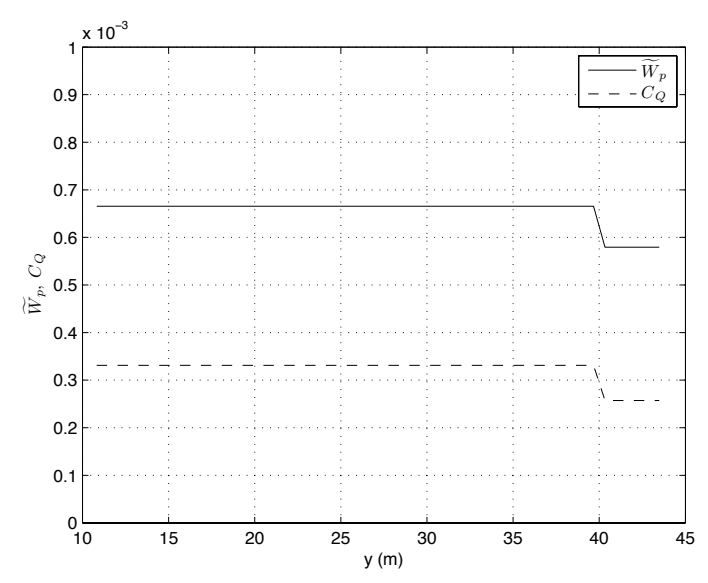

(b) Outboard

Figure 6.17: Variation in overall suction coefficient and pump drag coefficient along the wing half-span. $C_{Q}$ is $3.10 \times 10^{-4}$ and $\widetilde{W}_{p}$ is $6.27 \times 10^{-4}-R e_{u}=8 \times 10^{6} \mathrm{~m}^{-1}, \eta_{p}=0.85$.

for an evaluation of the mission performance, a detailed assessment of the propulsion system characteristics is necessary.

Cumpsty (1997) explains that engines are typically sized for conditions at top-of-climb and, under normal conditions, provide ample thrust at takeoff. In contrast, the open literature (see Chap. 2) suggests that takeoff is a more critical condition for laminarised aircraft. Furthermore, with a cruise Mach number of 0.67 , the prospect of turboprop engine propulsion is a possibility. Therefore, in this chapter, a power-plant scoping study is undertaken to compare the relative performances of turbofan and turboprop engines, over a range of design points, following the design methodology proposed in Chap. 3. Configurations that provide satisfactory operation are compared on the basis of specific fuel consumption in cruise with suction.

\subsubsection{Thrust and Power Requirements}

For an aircraft with a flight path angle $\gamma$ and mass $M$, the thrust required to react the weight and drag forces is

$$
F_{N}=M g\left(\sin \gamma+\frac{\cos \gamma}{L / D}\right)
$$

Mair and Birdsall (1992) specify that the most demanding takeoff flight segment for the engines is at an altitude of around $120 \mathrm{~m}$, with the undercarriage retracted, and making allowance for the failure of one engine. In this study, two and three-engine configurations are 


\subsection{LAMINAR-FLYING-WING DESIGN CONCEPT}

considered; the corresponding minimum climb-out angles are $1.38^{\circ}$ and $1.55^{\circ}$, respectively (Mair and Birdsall, 1992). At cruising altitude, the minimum rate of climb is usually taken as 300 feet per minute, which translates to a flight-path angle of around $0.5^{\circ}$. In cruise, the flight-path angle is taken as zero.

For the purposes of the power-plant scoping study, four operating conditions are considered: top-of-climb (with suction), takeoff (without suction) with one engine inoperative, and beginning of cruise with/without suction. (End of cruise is considered in Sec. 6.1.4.3 once an appropriate power plant has been identified.)

\subsubsection{Power-Plant Scoping Study}

Table 6.7 summarises the requirements, per engine, over the range of operating conditions for both two and three-engined configurations. (Note, the thrust requirement for two engines is evaluated based on the calculated $L / D$ for three engines.) At takeoff, the required thrust is strongly dependent on the weight of the aircraft, rather than its aerodynamic performance. Therefore, the obvious design challenge is that around a ninth of the takeoff thrust is needed in laminarised cruise for a two-engined aircraft, though this is slightly higher, at around a seventh, for a three-engined aircraft.

Table 6.7: Thrust and suction power requirements (per engine) at various flight conditions for two/three engines.

\begin{tabular}{llclc}
\hline \hline Parameter & \multicolumn{3}{c}{ Suction } & \multicolumn{2}{c}{ No suction } \\
& TOC & Begin cruise & Begin cruise & Takeoff (engine out) \\
\hline Altitude $(\mathrm{m})$ & 6858 & 6858 & 6858 & 120 \\
$M_{\infty}$ & 0.67 & 0.67 & 0.39 & 0.21 \\
$F_{N}(\mathrm{kN})$ & $23.1 / 15.4$ & $15.0 / 10.0$ & $37.1 / 24.7$ & $126.1 / 65.7$ \\
PWX $(\mathrm{kW})$ & $983 / 672$ & $983 / 672$ & - & - \\
\hline \hline
\end{tabular}

\section{Turbofan}

Two engines

Taking the design point at TOC, an engine with a BPR of four gives satisfactory jet velocity ratios for all operating conditions. In an attempt to alleviate conditions off-design, the compressor and burner exit temperatures are reduced with $T_{03}=700 \mathrm{~K}$ and $T_{04}=1500 \mathrm{~K}$. The HPC map, detailing the design point and the operating line for increasing engine speed at takeoff (TO) conditions, is shown in Fig. 6.18. The HPC pressure ratio at the design point is around nine. The overall pressure ratio (OPR) is around 40, which is comparable to 
typical OPRs detailed in Cumpsty (1997). In contrast, much higher HPC pressure ratios are expected at TO.

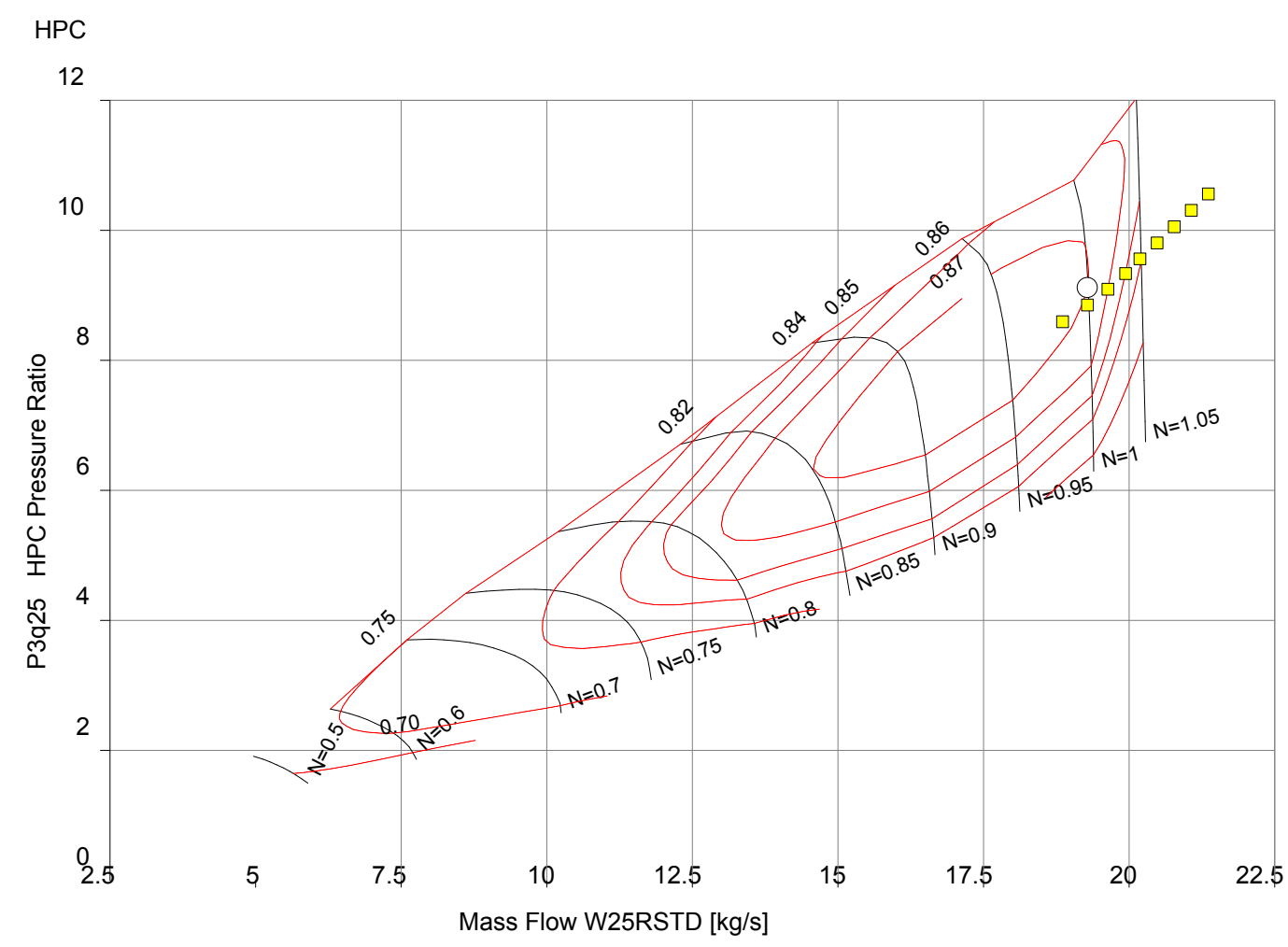

Figure 6.18: Two engine turbofan HPC operating map, detailing the TOC design point (white circle), and takeoff off-design point for increasing engine mass flow rate (yellow squares). The non-dimensional spool speed $(\mathrm{N})$ is represented by the solid black contours, and the compressor efficiency by the red contours.

Figure 6.19 details the net thrust, HPC exit temperature and burner exit temperature variation with engine mass flow rate at TO conditions (with the design point at TOC). The maximum available thrust is below $72 \mathrm{kN}$, whilst both $T_{03}$ and $T_{04}$ breach the limits imposed in this study. This typically critical design condition therefore falls short of providing the required thrust at TO.

Consider now the HPC operating map shown in Fig. 6.20 with takeoff as the design condition, for an engine with a bypass ratio of six. Clearly takeoff is the most demanding operating condition of the three detailed. (Note, TOC is not an extreme operating condition, and consequently is not shown.) To maximise engine efficiency in cruise, $T_{03}$ and $T_{04}$ at TO are set to the maximum values, i.e. $900 \mathrm{~K}$ and $1800 \mathrm{~K}$, respectively. Due to the large 


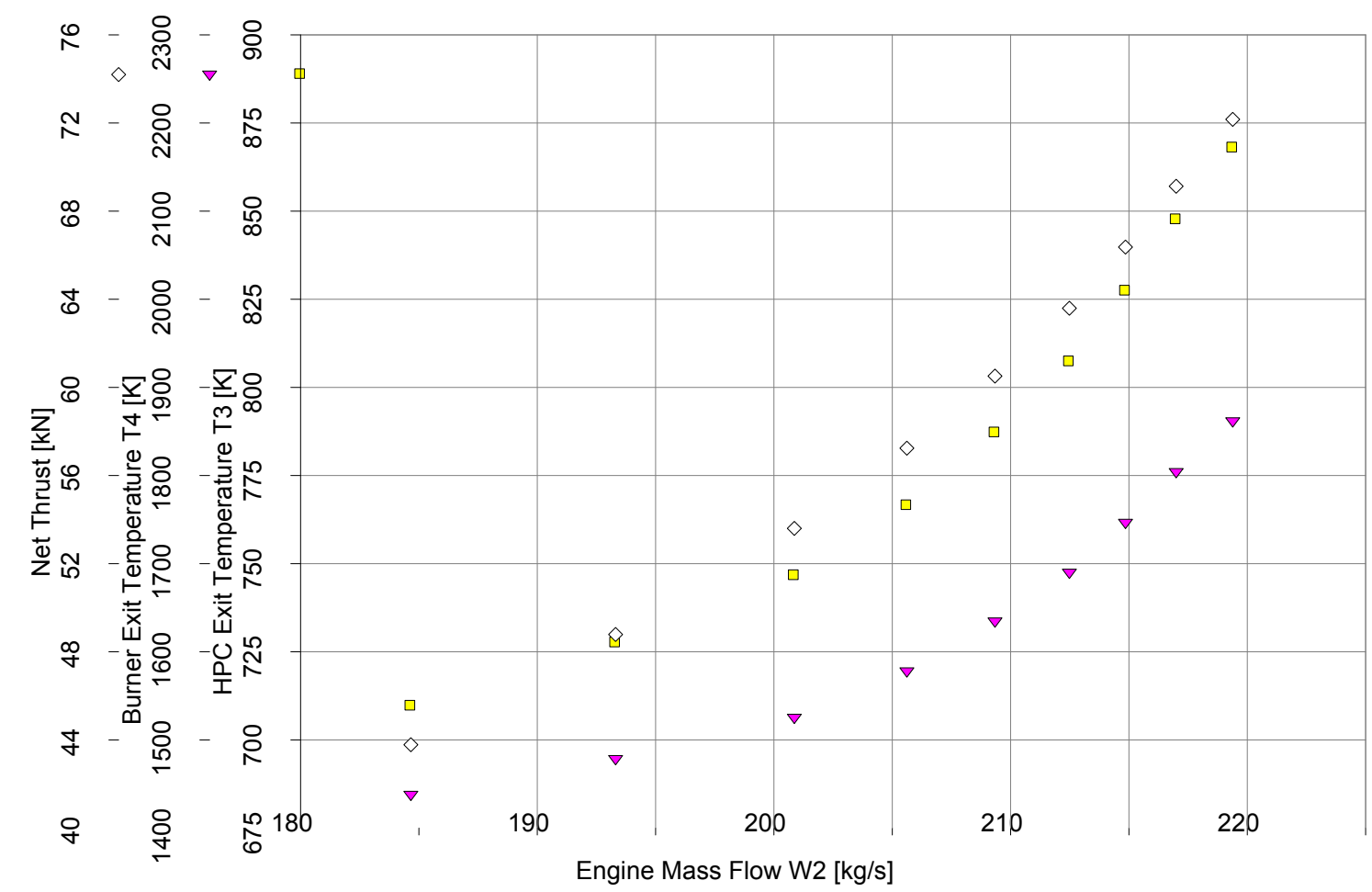

Figure 6.19: Net thrust, HPC exit temperature and burner exit temperature variation with engine mass flow rate at TO conditions for a two-engine turbofan designed for TOC.

variation in thrust requirement across the flight envelope, the HPC map reveals that the operating points are much more widely spaced than is typical, with cruise efficiency reduced below typical values. Moreover, in an attempt to improve cruise efficiency and move this point away from the surge/stall line, the design point on the operating map, at the cost of a lower compressor efficiency, has been rescaled - an insignificant compromise, given it represents a small fraction of the total flight time. The resulting $s f c$ for cruise with suction is $23.0 \mathrm{~g} / \mathrm{kN} . \mathrm{s}$

In an attempt to improve cruise efficiency, taking cruise without suction as the design point was considered. The engine $s f c$ for laminarised cruise was found to improve, but at the cost of high burner exit temperatures and high relative HPC/LPC non-dimensional spool speeds at takeoff. 


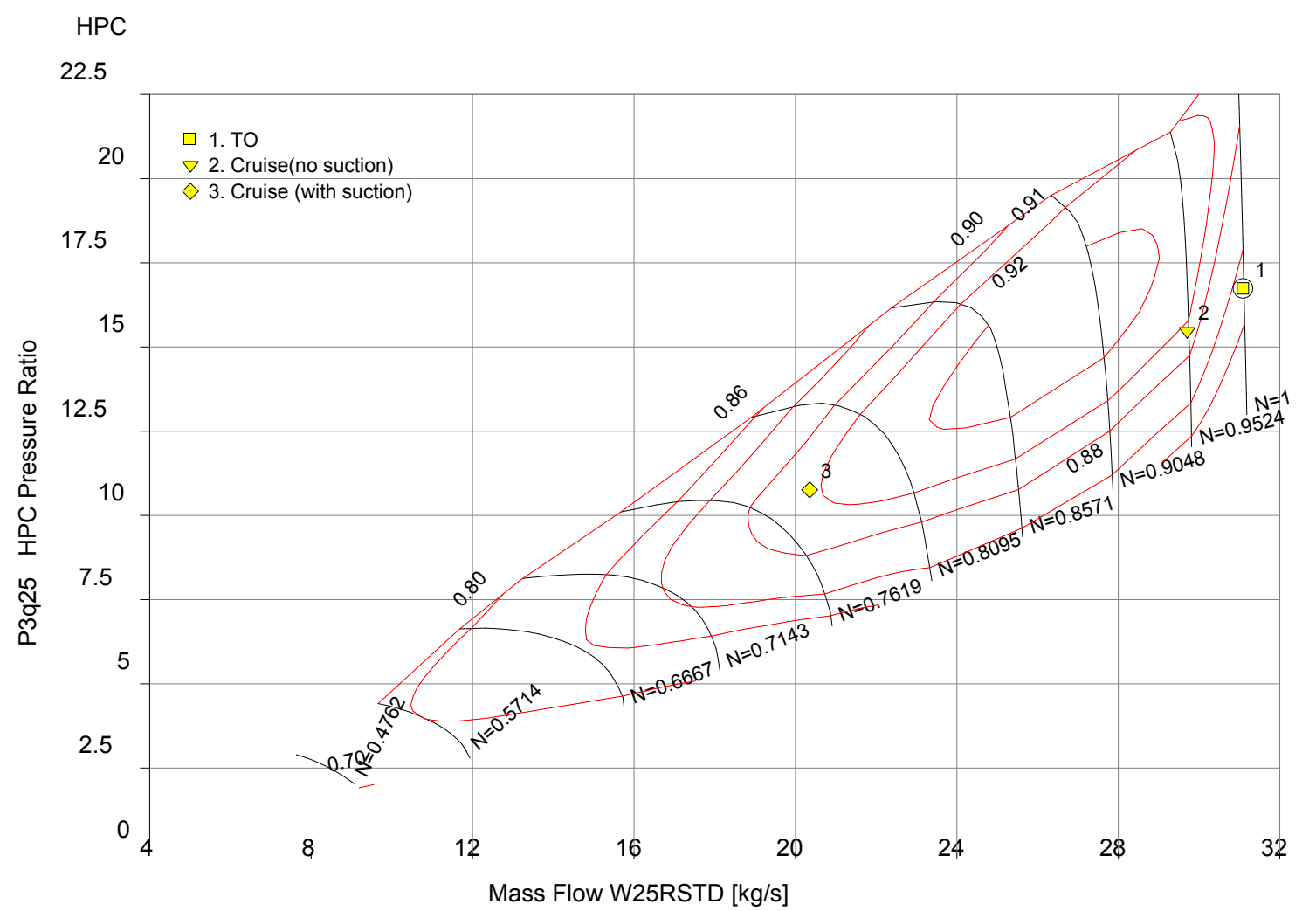

Figure 6.20: Two engine turbofan HPC operating map, detailing the TO design point (white circle), and various off-design points (yellow squares). The non-dimensional spool speed (N) is represented by the solid black contours, and the compressor efficiency by the red contours. The design point has been rescaled with $\beta=0.3$ and $N=1.05$.

\section{Three engines}

As for the two engine case, attempts to maximise efficiency in cruise with suction by taking the design point at TOC were faced with a lack of available thrust at takeoff. Therefore, alternative design points are considered below.

Figure 6.21 shows the HPC operating map for an engine with a BPR of five and cruise without suction as the design point. Despite TO and the design point being very closely spaced, compromised values for $T_{03}$ and $T_{04}$ of $700 \mathrm{~K}$ and $1500 \mathrm{~K}$, respectively, were necessary to alleviate conditions for the former. As for the two-engine case, the compressor efficiency at TO was compromised by rescaling the map, primarily to better improve the efficiency at cruise with suction. Similarly, the takeoff and cruise points are widely spaced. The estimated $s f c$ for cruise with suction is $23.0 \mathrm{~g} / \mathrm{kN}$.s. 


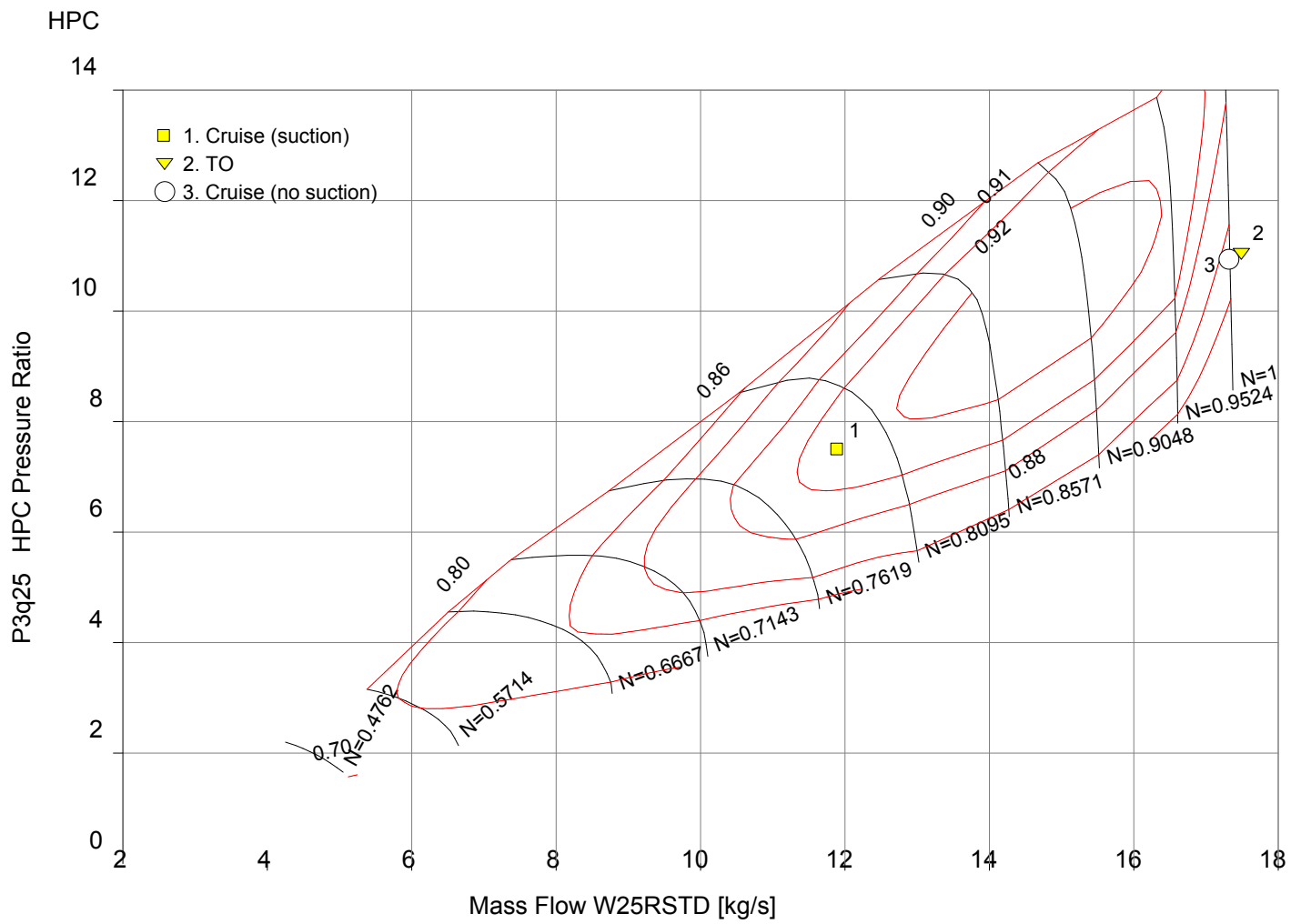

Figure 6.21: Three engine turbofan HPC operating map, detailing the cruise-withoutsuction design point (white circle), and various off-design points (yellow squares). The non-dimensional spool speed $(\mathrm{N})$ is represented by the solid black contours, and the compressor efficiency by the red contours. The design point has been rescaled with $\beta=0.3$ and $N=1.05$.

\section{Turboprop}

Two engines

As for the two engine turbofan, preliminary investigations showed that the turboprop suffers from a lack of available thrust at takeoff when taking the design point at top-of-climb. However, a different problem arises with TO as the design point: the turboprop suffers from a high thrust coefficient. GasTurb has a threshold of $C_{T}=0.5$ : a $5.6 \mathrm{~m}$ diameter blade rotating at a speed of $853 \mathrm{RPM}$, corresponding to a blade tip speed of $250 \mathrm{~m} / \mathrm{s}$, puts the thrust coefficient at this limit. Such a low rotational speed posits various problems, whilst GasTurb places an upper limit of $5 \mathrm{~m}$ on blade diameter. Despite accepting slightly higher blade tip speeds, satisfactory thrust coefficient values remained difficult to obtain. 


\section{CHAPTER 6. CONCEPTUAL DESIGN STUDY}

\section{Three engines}

With three engines the thrust requirement at takeoff per engine is reduced. Consequently, a blade diameter of $4.15 \mathrm{~m}$ rotating at $1150 \mathrm{RPM}$ (blade tip speed of $250 \mathrm{~m} / \mathrm{s}$ ) is permissible without encountering high thrust coefficients. The propeller map over the range of operating conditions is detailed in Fig. 6.22.

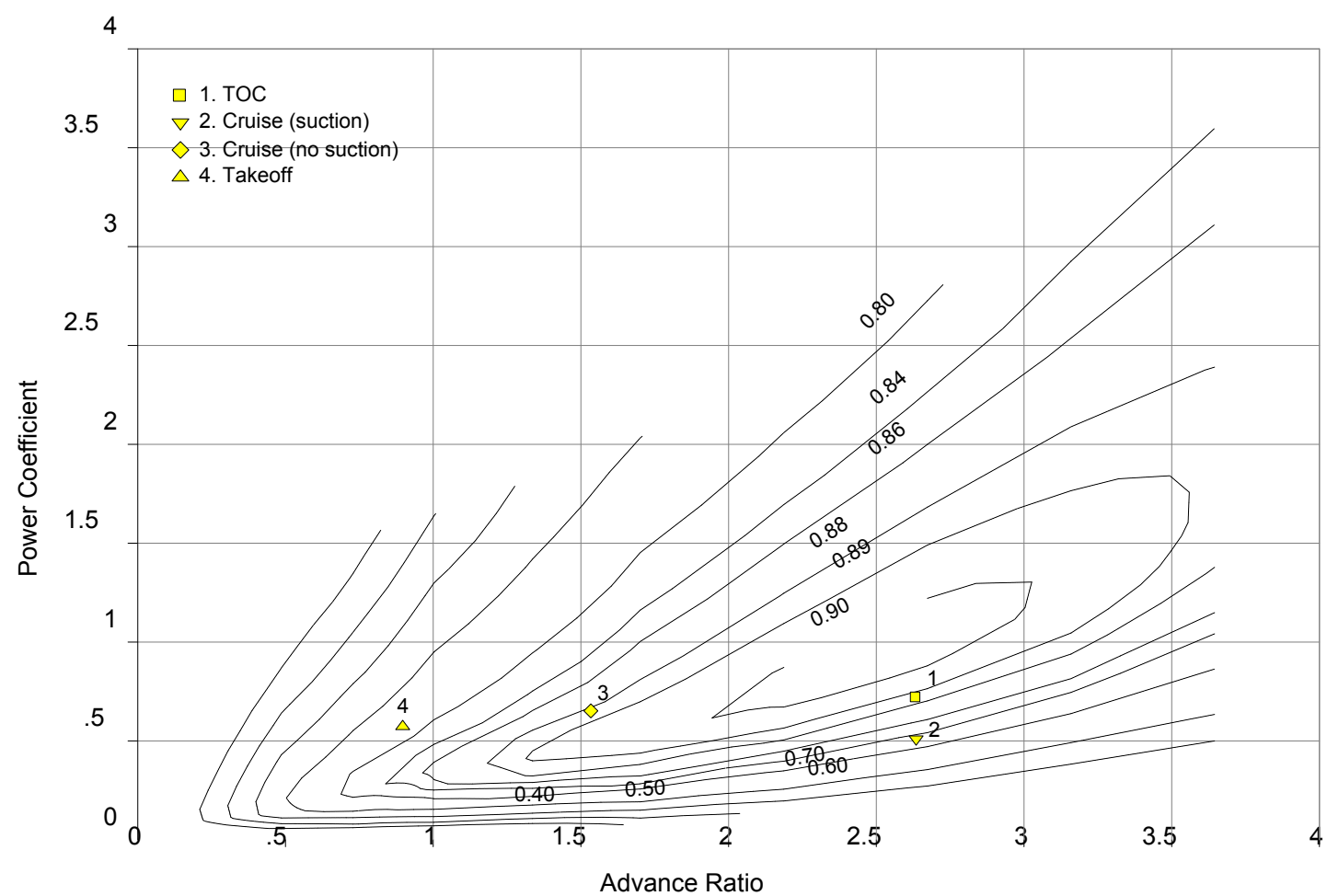

Figure 6.22: Propeller map for a three-engine turboprop with TO as the design point. (Solid black lines represent contours of propeller efficiency.)

The compressor operating map shown in Fig. 6.23 corresponds to an engine designed at TO. The map has been rescaled to alleviate conditions in cruise with suction, whilst also improve efficiency. Cruise without suction operates at the highest non-dimensional spool speed, and as a consequence, design point values for $T_{03}$ and $T_{04}$ of $700 \mathrm{~K}$ and $1600 \mathrm{~K}$ were selected in order to avoid compromising the viability of the design. The maximum compressor pressure ratios are also encountered at this condition, with a value of around 18 . (Saravanamuttoo (1987) notes that turboprop engines typically operate between a value of $16-25$.) The calculated $s f c$ for cruise with suction is $21.5 \mathrm{~g} / \mathrm{kN} . \mathrm{s}$.

Further consideration of alternative design points is not pursued. This is because taking 


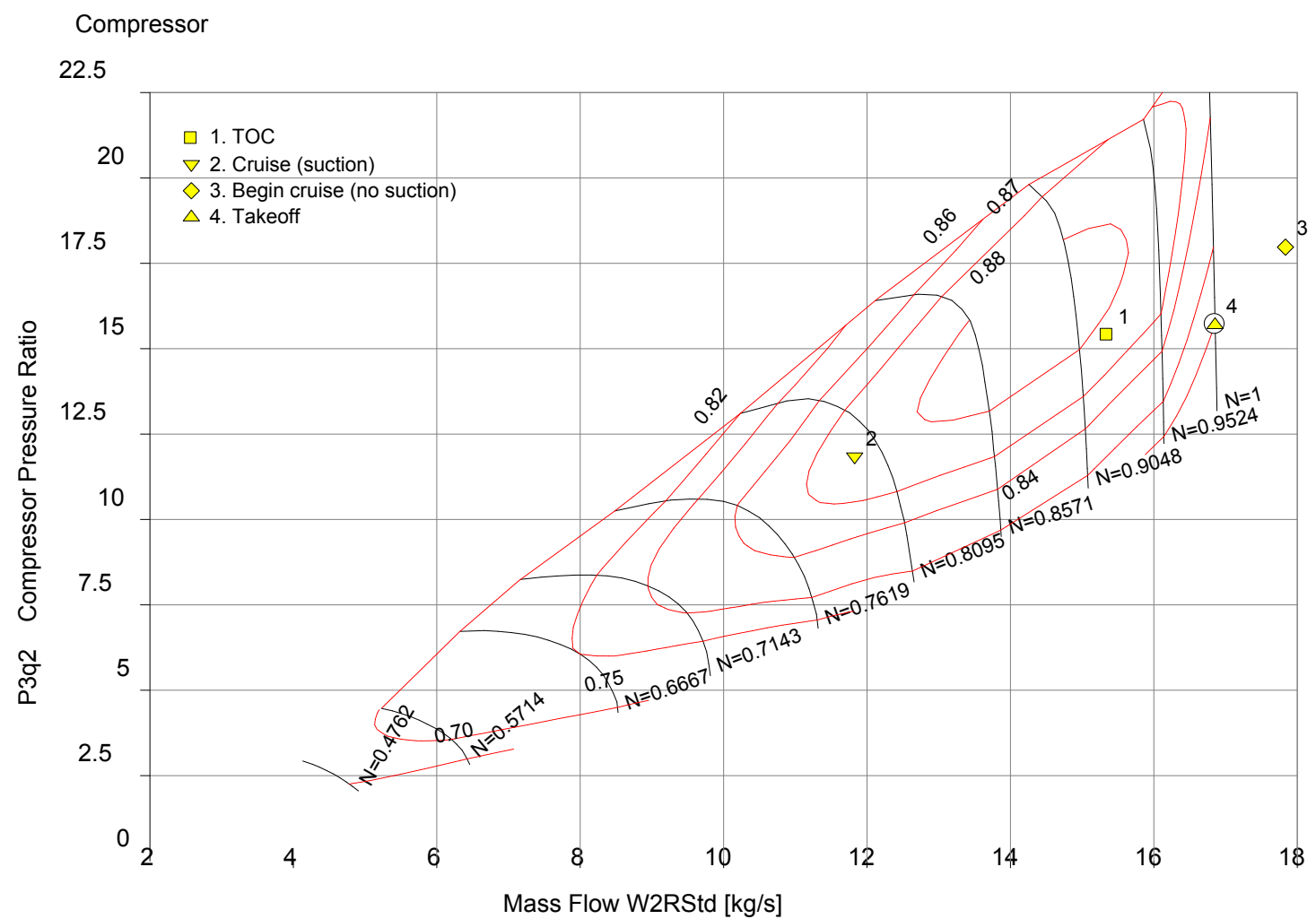

Figure 6.23: Three engine turboprop HPC operating map, detailing the TO design point (white circle), and various off-design points (yellow squares). The non-dimensional spool speed $(\mathrm{N})$ is represented by the solid black contours, and the compressor efficiency by the red contours. The design point has been rescaled with $\beta=0.2$ and $N=1.05$.

cruise-without-suction as the design point will severely compromise compressor efficiency in laminarised cruise, whilst TOC as the design point will compromise the viability of the design at takeoff and cruise-without-suction.

\section{Discussion}

The power-plant scoping study has examined the application of turboprop and turbofan engines to the LFW aircraft for the following operating conditions: top-of-climb with suction, takeoff, and cruise with/without suction. The investigation revealed that the engine operating characteristics differ significantly from those utilised by conventional aircraft, primarily due to the large difference in thrust requirement between takeoff and laminarised cruise.

First two turbofan engines were investigated. Taking TOC as the design point, to max- 


\section{CHAPTER 6. CONCEPTUAL DESIGN STUDY}

imise efficiency in cruise with suction, the thrust generated at takeoff was found to be insufficient. Designing for TO gave a viable engine design with a $s f c$ of $23.0 \mathrm{~g} / \mathrm{kN}$.s for cruise with suction. Finally, designing for cruise without suction to improve $s f c$ resulted in extreme conditions at takeoff. With three turbofan engines, the thrust requirement at takeoff per engine reduced; however, fixing the engine size at TOC still resulted in a design that was incapable of generating the required thrust at takeoff. Designing for cruise without suction gave the best $s f c$ for this configuration, $23.0 \mathrm{~g} / \mathrm{kN}$.s. There appear, therefore, to be no benefits from adopting a three-engine turbofan configuration over a two-engine one.

Next turboprops were considered. A configuration based on two engines with the design condition at TOC with suction, like the turbofan, suffered from inadequate thrust generation at TO. Takeoff as the design point was then investigated. Due to the high thrust requirement, overly high thrust coefficients were unavoidable. Therefore, three engines were considered. A blade diameter of $4.15 \mathrm{~m}$ with a rotational speed of 1150 RPM avoided an excessively high thrust coefficient. Like the turbofan, TOC as the design point produced an engine incapable of achieving the TO thrust required. Design at takeoff gave the best cruise-with-suction $s f c$ of $21.5 \mathrm{~g} / \mathrm{kN} . \mathrm{s}$, which is $7 \%$ lower than the best turbofan design.

\subsubsection{LFW Engine Performance}

On the basis of the power-plant scoping study, a three-engine turboprop offers the best solution for the laminar flying wing. Table 6.8 summarises the requirements per engine over the full range of flight conditions needed for estimation of the aircraft's mission range. Start and end of cruise conditions are calculated based on a cruise-climb flight path. The change in $L / D$ is assumed to be negligible. However, a change in altitude and flight speed affects the unit Reynolds number, and hence suction power requirement. For constant values of: thickness-to-chord ratio, Mach number and $C_{L}$, the increase in Reynolds number, here, translates to just under a $7 \%$ increase in power (evaluated based on the parametric study presented in Chap. 4). Should suction not be available at top-of-climb, the mission would be aborted; therefore, only cruise is considered for the suction-failure case.

The HPC map with takeoff as the design point is shown in Fig. 6.24. It reveals that in the event of suction failure, cruise is the more critical condition with a pressure ratio of around 20. However, designing for this condition would further compromise engine efficiency when suction is employed. Note, as in the scoping study, the design point has been rescaled to ensure operation with suction does not result in an engine surge/stall, and also to improve the efficiency at this condition without encountering problems for cruise without suction.

The engine design specification is summarised in Tab. 6.9. A propeller diameter of 4.15 
Table 6.8: Thrust and suction power requirements (per engine) at various flight conditions.

\begin{tabular}{|c|c|c|c|c|c|c|}
\hline \multirow[t]{2}{*}{ Parameter } & \multicolumn{3}{|c|}{ Suction } & \multicolumn{3}{|c|}{ No suction } \\
\hline & TOC & Begin cruise & $\begin{array}{l}\text { End } \\
\text { cruise }\end{array}$ & Begin cruise & $\begin{array}{l}\text { End } \\
\text { cruise }\end{array}$ & Takeoff (e/o) \\
\hline Altitude (m) & 6858 & 6858 & 8717 & 6858 & 8137 & 120 \\
\hline$M_{\infty}$ & 0.67 & 0.67 & 0.67 & 0.39 & 0.39 & 0.21 \\
\hline$F_{N}(\mathrm{kN})$ & 15.4 & 10 & 8.4 & 24.7 & 20.7 & 65.7 \\
\hline PWX $(\mathrm{kW})$ & 672 & 672 & 715 & - & - & - \\
\hline
\end{tabular}

Compressor

22.5

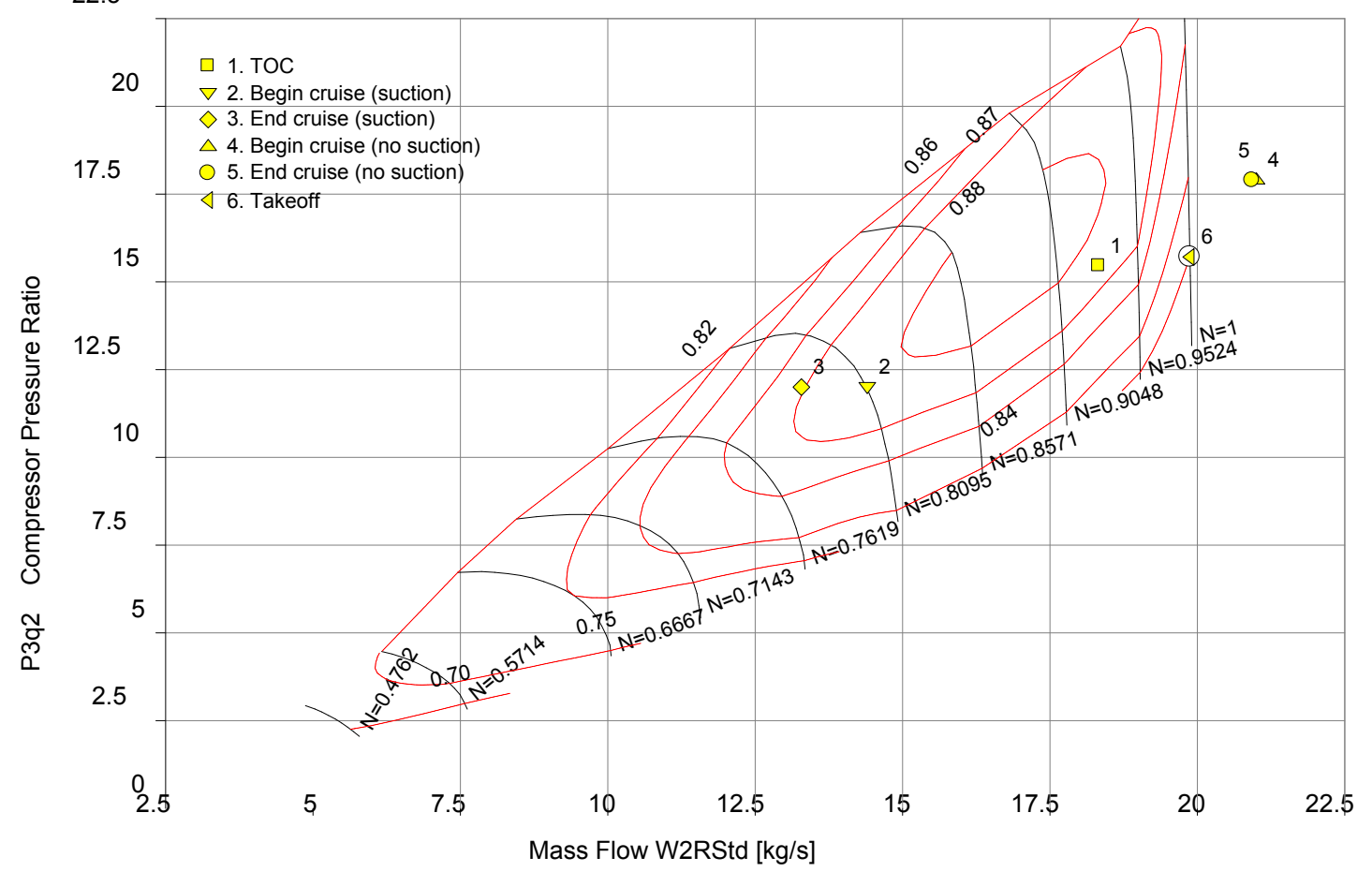

Figure 6.24: HPC operating map detailing the takeoff design point, various off-design points, non-dimensional spool speed (N) contours (solid black), and efficiency contours (red). The design point has been rescaled with $\beta=0.2$ and $N=1.05$.

$\mathrm{m}$ and rotational speed of $1150 \mathrm{RPM}$ avoids excessive thrust coefficients and power loadings at takeoff, with values of 0.49 and $351 \mathrm{~kW} / \mathrm{m}^{2}$, respectively. Furthermore, the advance ratio and power coefficients operate within a range of $0.90-2.64$ and $0.51-0.80$, respectively. The compressor and burner exit temperatures at the design point are $700 \mathrm{~K}$ and $1500 \mathrm{~K}$ - the latter reduced, c.f. $1600 \mathrm{~K}$ in the scoping study, to alleviate values at end of cruise 
with suction. A propeller shaft power of around $6045 \mathrm{~kW}$ is required at takeoff. The overall efficiency in cruise ranges between $20-22 \%$. The calculated $s f c$ at start of cruise with suction is $22.0 \mathrm{~g} / \mathrm{kN} . \mathrm{s}$, increasing to $23.2 \mathrm{~g} / \mathrm{kN}$.s at end of cruise.

To put the engine specification into perspective, consider the Europrop TP400 ${ }^{1}$ used to power the Airbus A400M. This engine delivers a shaft power of $8203 \mathrm{~kW}$ on takeoff, which is $37 \%$ more than that for the LFW. The propeller consists of eight blades having a diameter of $5.3 \mathrm{~m}$, compared to only $4.15 \mathrm{~m}$ for the LFW; its power loading is $17 \%$ lower at $292 \mathrm{~kW} / \mathrm{m}^{2}$. It has a higher overall pressure ratio of 25 , which, however, is split between an intermediate and a high pressure compressor, with ratios of 3.5 and 7.2 , respectively.

GasTurb predicts a total mass of around $300 \mathrm{~kg}$. However, many details of a real engine are not modelled explicitly by GasTurb, and therefore the calculated mass is on the low side 2. The TP400 and NK-12 (see Saravanamuttoo (1987)) turboprop engines have dry-weightto-takeoff-power ratios of $0.23 \mathrm{~kg} / \mathrm{kW}$ and $0.26 \mathrm{~kg} / \mathrm{kW}$, respectively. Therefore, assuming the dry engine weight scales directly with shaft power delivered at takeoff, the estimated dry weight per engine is around $1500 \mathrm{~kg}$ here.

\footnotetext{
${ }^{1}$ Information taken from www.roll-royce.com and www.europrop-int.com, October 2011

${ }^{2}$ Detailed in the GasTurb manual - www.gasturb.de.
} 


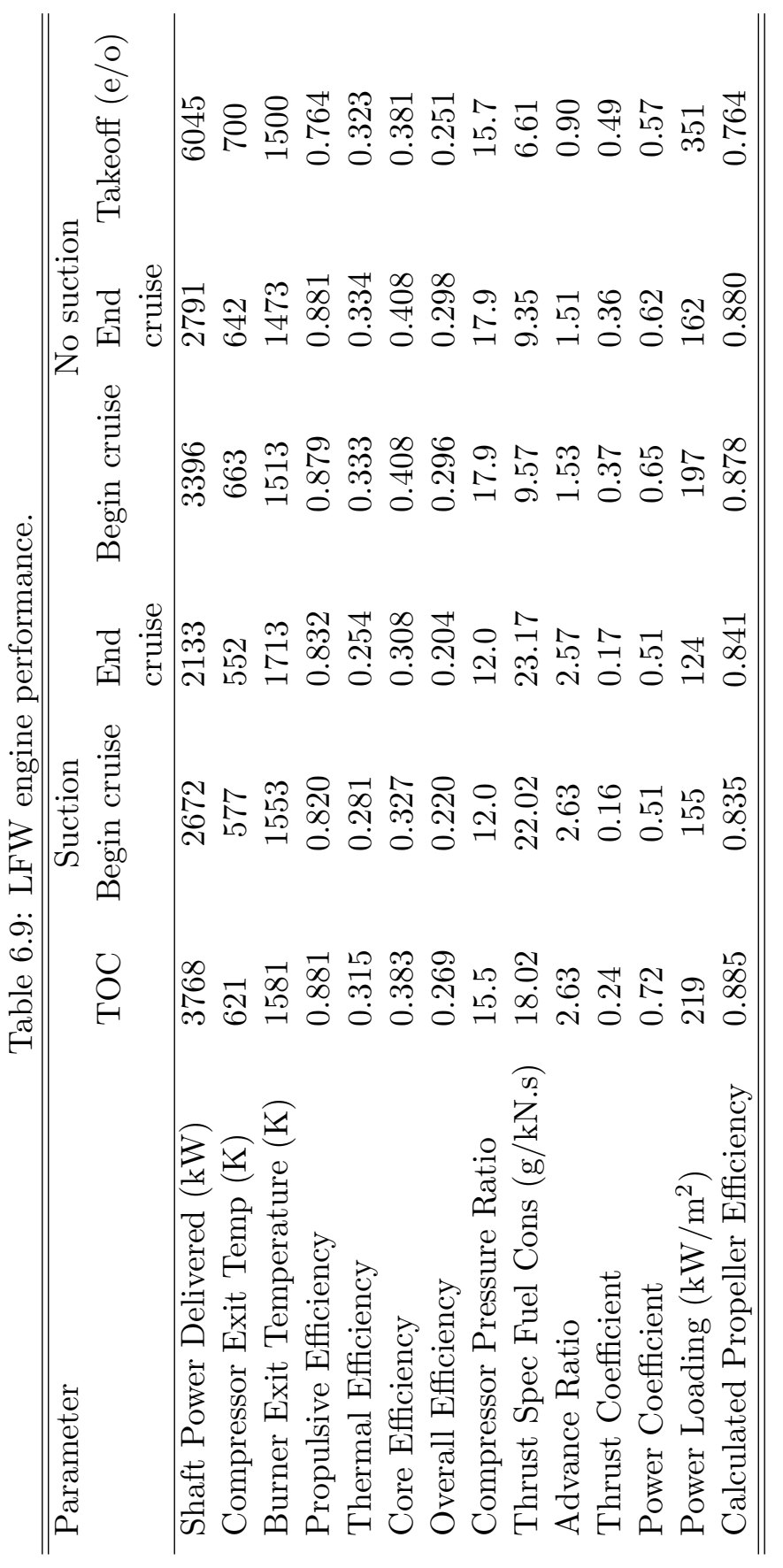




\subsubsection{Sensitivity Study}

With a large difference in thrust requirement between laminarised cruise and takeoff, the engine $s f c$ is compromised. Figure 6.25 details the variation of engine $s f c$ in cruise (with suction) as the engine speed, and hence thrust, is increased. It shows that, with a $36 \%$ increase in thrust, i.e. a $26 \%$ reduction in lift-to-drag ratio, the reduction in $s f c$ is about $14 \%$ (an increase in $\eta_{o v}$ from $22 \%$ to $25 \%$ ). Further improvements in $s f c$ begin to decay above $13.6 \mathrm{kN}$. Using the Breguet-range equation, a $26 \%$ reduction in $L / D$ thus translates to around a $14 \%$ reduction in range (for a fixed fuel weight and flight speed).

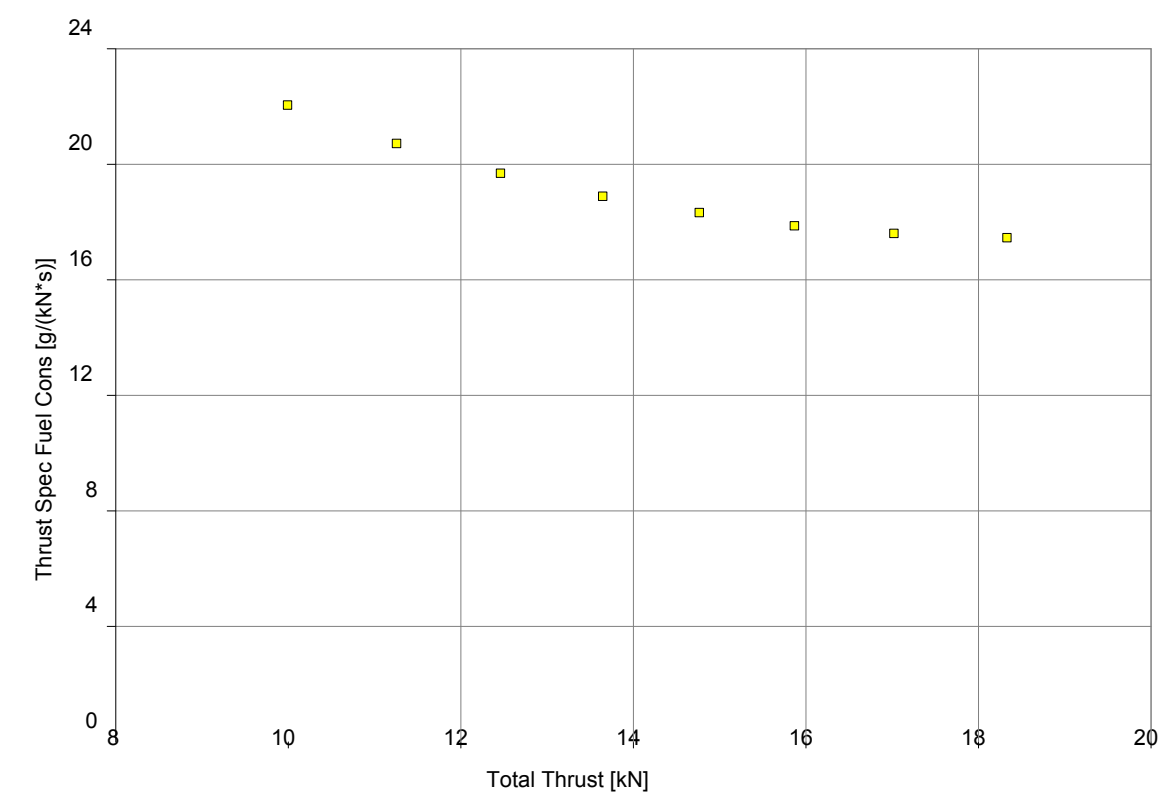

Figure 6.25: Variation of cruise (with suction) engine $s f c$ with thrust.

\subsubsection{Structures and Weights}

The structures and weights analysis consists of sizing primary structural elements for a given loading distribution and airframe geometry. Amongst other system loads (introduced later), the weights and locations of the passengers, suction pumps and engines are needed. The weight buildup and C.G. location are necessary for the airframe generation (Sec. 6.1.1), aircraft performance (Sec. 6.1.2), and determination of available fuel weight.

The structural components are sized using simple theory detailed in Chap. 3. The main construction material is assumed to be an aluminium alloy, 2024-T3 (Niu (1988)). 


\subsection{LAMINAR-FLYING-WING DESIGN CONCEPT}

\subsubsection{Structural Configuration}

The fuselage structure is modelled as a multi-bubble arrangement. It consists of several circular cylinders combined together and joined by vertical bulkheads to make an extended structure. The vertical bulkheads have cut-outs incorporated into them to allow access between adjoining cylinders, and passage throughout the cabin. Both frames and stringers stabilise against potential local buckling and provide extra structural rigidity (e.g. in the event of a low speed collision); frames also serve to transmit local shear loads to the wing structure. However, it is proposed that wing ribs also double-up as frames. A cabin floor is required to support the payload and seats etc. Pressure bulkheads cap off the spanwise extremes of the cabin from the unpressurised regions. A portion of the fuselage is detailed in Fig. 6.26 - the fuselage would consist of several such arrangements in the spanwise direction.

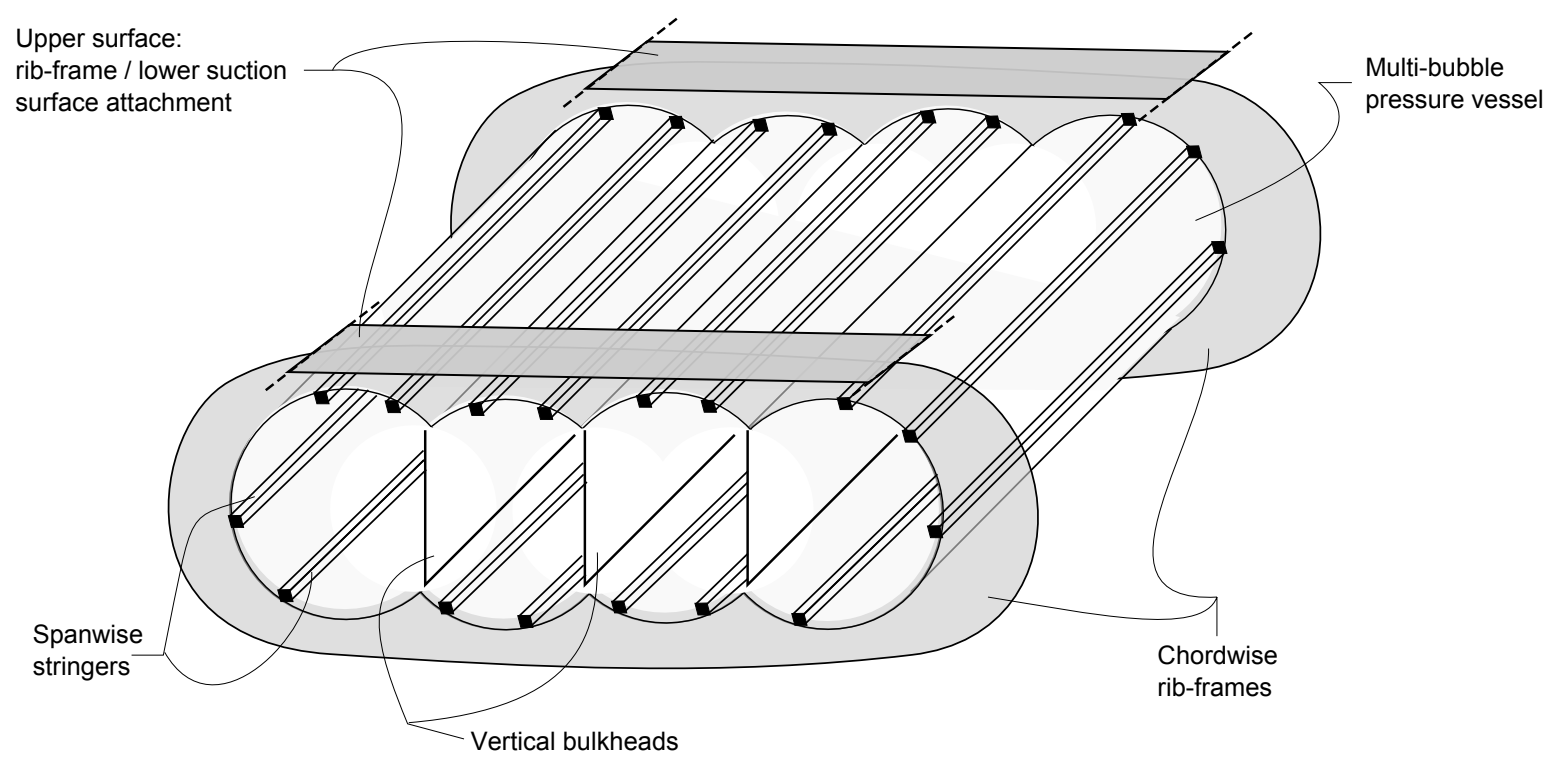

Figure 6.26: Schematic diagram of LFW fuselage structural arrangement.

Geuskens et al. (2008) propose that segregation of the cabin from the wing structure eliminates the appearance of large stresses in the wing structure. Furthermore, with the pressure vessel supported by wing ribs, and the connections assumed pinned, negligible bending moments are likely to be incurred due to self weight. For preliminary design purposes, the cabin and wing structures are assumed to be effectively decoupled. Therefore, the cabin shell is analysed subject to pressure loads alone.

The LFW is assumed to have a conventional wing structure, consisting of four main elements: the wing skin, stringers, ribs and spar-webs. The chambers are integrated into 
the distributed flange construction by allowing the top of the chamber (the wing skin) and its bottom plate to resist bending loads, whilst the chamber walls act as stiffeners/stringers. Note, the wing skin thickness is set at $1 \mathrm{~mm}$, regardless of loading, to avoid manufacturing issues with a porous skin (Inger and Babinsky (2000)).

A schematic diagram of the wing and wingtip-fin geometries is provided in Fig. 6.27. Four spanwise reference stations are highlighted: 1) wing root, 2) cabin boundary, 3) outboard wing/wingtip-fin intersection, and 4) wingtip fin edge. The front and rear spars of the wing-box are positioned at $10 \%$ and $70 \%$ chord along the outboard regions; however, across the centrebody they have a fixed distance from the nose of the aircraft. The reference axis coincides with the locus of the shear centre of the wing box. The outboard axis has a non-zero sweep angle. With the front and rear spars aligned parallel to the centrebody trailing edge, a kink in the wing-box construction results at station 2. A rectangular wing-box construction is assumed, as detailed by Howe (2004), with depths, selected on the basis of providing accommodation for the placement of suction equipment and structural reinforcements, of: 1) $2.3 \mathrm{~m}, 2) 2.2 \mathrm{~m}$, and 3) $1.0 \mathrm{~m}$.

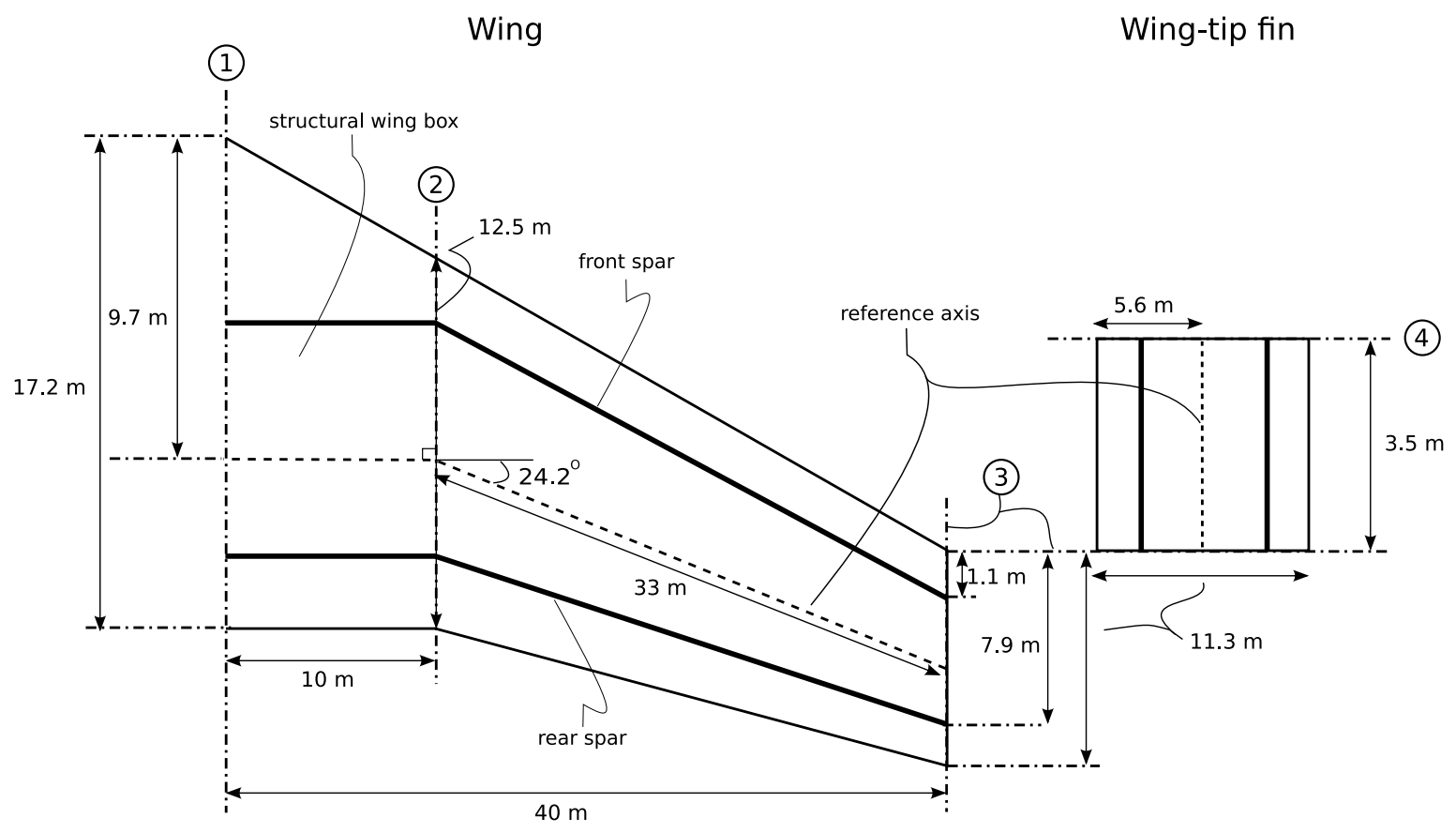

Figure 6.27: Wing structural geometry. 


\subsection{LAMINAR-FLYING-WING DESIGN CONCEPT}

\subsubsection{System Loads Distribution}

Figure 6.28 illustrates: a) the regions over which the imposed loads act, and the critical load combinations for b) in-flight and c) on ground. Structural, payload, baggage and fuel weight are distributed between the front and rear spars; payload occupies the centrebody regions, whilst baggage and fuel are placed outboard with their spanwise extent limited by minimum volume requirements. (A volume per bag of $0.3 \mathrm{~m}^{3}$ and a factor of 1.5 applied to the total number of passengers gives a total baggage volume requirement of $99 \mathrm{~m}^{3}$; jet fuel has a density of around $750 \mathrm{~kg} / \mathrm{m}^{3}$, translating to a fuel tank volume requirement of $40 \mathrm{~m}^{3}$. 1) Baggage storage is placed further inboard relative to the fuel tank for passenger safety. The aero-loading is shown as a distributed pressure force $p(y)$ (see Sec. 6.1.2). The engine, auxiliary power units (APU), suction hardware, avionics, nose wheel and undercarriage are modelled as point loads. The ground reaction force $R$ acts as a point load through the main undercarriage, which is arbitrarily positioned at $75 \%$ chord at the spanwise extremes of the centrebody (the landing gear arrangement is discussed further in Sec. 6.1.5.5). With three engines having a blade diameter of $4.15 \mathrm{~m}$, the spacing is set at $7 \mathrm{~m}$. A minimum distance of $1 \mathrm{~m}$ is reserved either side of the cabin for suction ducting.

The maximum takeoff weight (MTOW) consists of the design payload, the operating empty weight (OEW), and the fuel weight. The design payload is defined as 220 persons in Sec. 6.1.1, each with a total weight allotment of $100 \mathrm{~kg}$ (including $20 \mathrm{~kg}$ for baggage). The OEW is created from estimates of structures, propulsion system, suction system, landing gear, and fixed equipment.

\subsubsection{Structural Analysis}

\section{Structural Forces and Moments}

The aero-loading is calculated at the cruise flight condition $\left(C_{L}=0.14\right.$ and $\left.M_{\infty}=0.67\right)$ with zero control surface deflection. It is scaled by the gust load factor for the low wing loading LFW, which is found to be more critical than a typical 2.5-g pull-up manoeuvre, at around 3.5 -g.

The structural forces that arise when the (initially unknown) self-weight is excluded are indicated by the solid lines in Fig. 6.29. Overall, along the span, the shear force increases going inboards. There is a distinct reduction in the slope over the centrebody region, due to the action of the system loads. The overall, negative, increase in bending moment and reduction in slope over the centrebody region may be attributed to the same factors as those

\footnotetext{
${ }^{1}$ www.bp.com - Air BP, handbook of products.
} 
a)

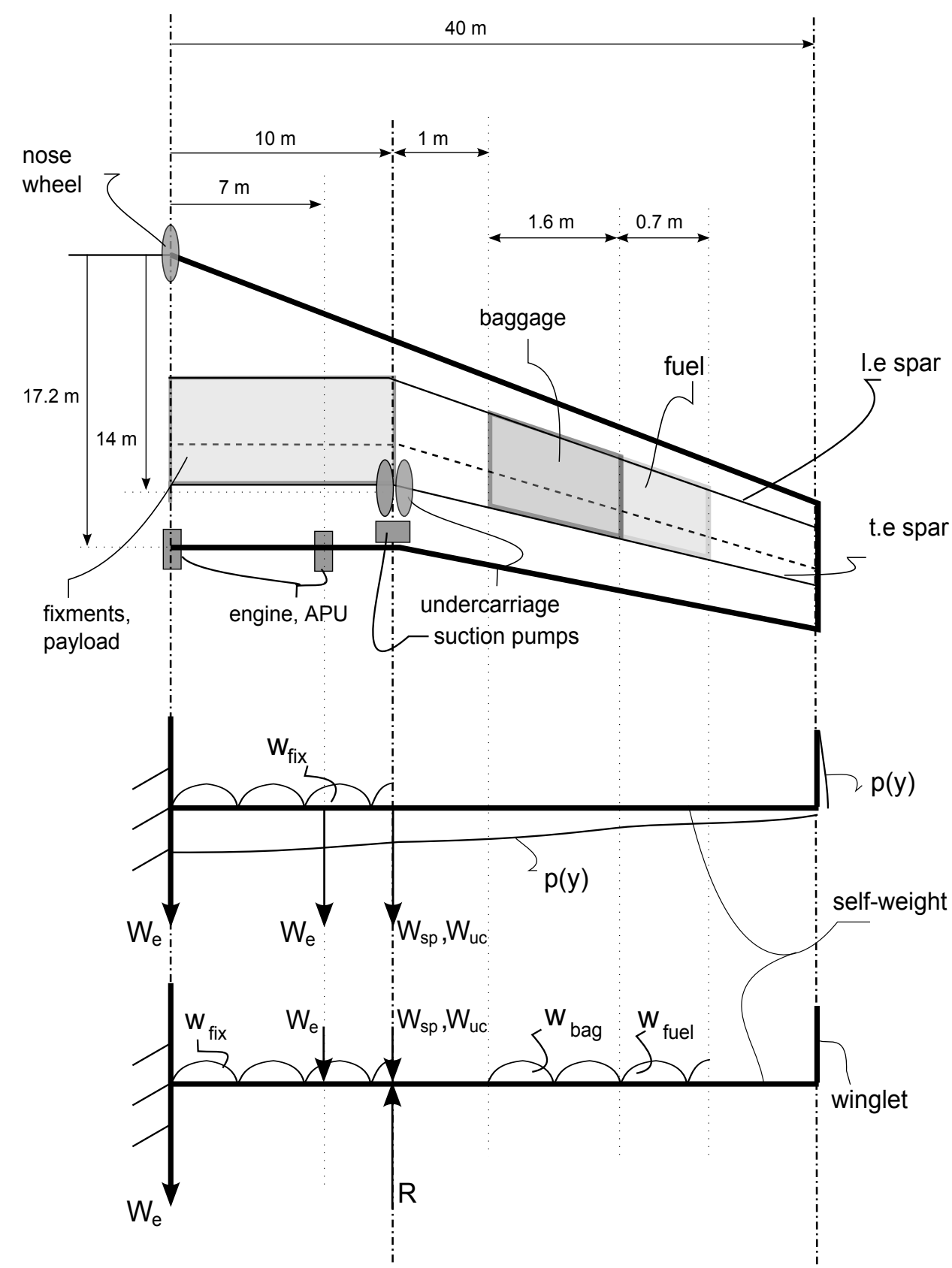

(1)

(2)

(3)

Figure 6.28: The distribution of weights and point loads over the planform is shown in a), whilst in b) and c) the root cantilever model is illustrated for the in-flight and on-ground load cases. 
for the shear force; the distinct jump is due to the resolution of moments across the sweep discontinuity. The increase in torque is physically consistent as the local lift acts forward of the shear centre of the outboard wing; whilst the distinct reduction across the sweep discontinuity, due to the resolution of forces, arises as the overall lift on the swept outboard section lies aft of the shear centre of the inboard section.
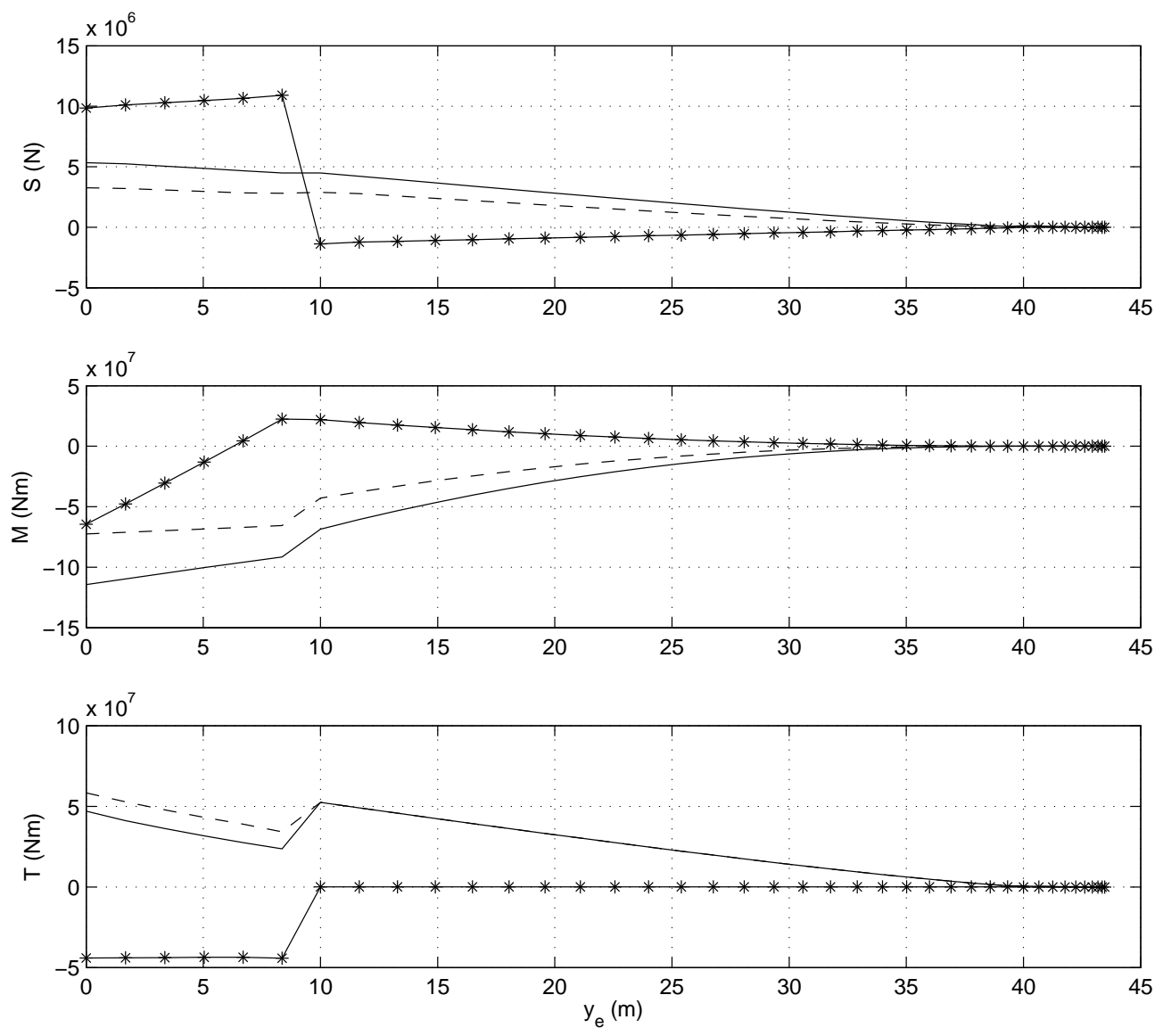

Figure 6.29: Shear force, bending moment and torque diagrams for critical load cases — initial estimate for in-flight (solid line), final estimate including self weight for in-flight (dashed line) and final estimate for ground (solid-star line).

The converged structural forces for the in-flight and ground regimes are indicated by the dashed and solid-star lines in Fig. 6.29, respectively. The in-flight loading calculation with self-weight included shows some bending relief, but the torque on the outboard section 


\section{CHAPTER 6. CONCEPTUAL DESIGN STUDY}

is unaffected due to the coincidence of centre of mass and shear centre. The reduction in the change in torque across the sweep discontinuity is due to the reduced bending moment. The ground forces reveal hogging on the outboard section, whilst sagging occurs over the centrebody region due to the action of the reaction force via the undercarriage. There is no torque over the outboard span as the baggage, fuel and structural weight C.G.s are taken to coincide with the wing shear centre. A negative torque arises on resolution of moments and torques across the sweep discontinuity due to the bending moment contribution; the step reduction in torque at $10 \mathrm{~m}$ is due to the eccentricity of the undercarriage force.

On comparing the ground and in-flight structural forces: the in-flight shear force at the inboard/outboard intersection is most critical outboard, in contrast to the ground shear force at the inboard/outboard intersection for the centrebody region; the in-flight bending moments and torque couples are most critical for sizing the structural elements.

\section{Material Thickness}

The fuselage skin and vertical/domed bulkheads each have thicknesses of $1.5 \mathrm{~mm}$. The cabin floor consists of a plank supported by $10 \mathrm{~cm}$ deep I-beams. The wing structure consists of: a main-wing skin with a mean effective thickness of $7 \mathrm{~mm}$ and $14 \mathrm{~mm}$ for the compressive and tensile surfaces, respectively; the mean wingtip-fin skin thickness is $2 \mathrm{~mm}$; the main-wing spar webs have a mean thickness of $12 \mathrm{~mm}$, whilst those corresponding to the wingtip-fins are 4 $\mathrm{mm}$; the rib webs have a thickness of $1 \mathrm{~mm}$, whilst only the ribs supporting the undercarriage have a significant spar thickness of around $12 \mathrm{~mm}$ - buckling requirements demand a rib pitch over the outboard and wingtip fin regions of $0.82 \mathrm{~m}$ and $0.55 \mathrm{~m}$, respectively, whilst the spacing over the cabin region is dictated by fuselage buckling requirements; the control surfaces have a mean spar-boom flange thickness of $4 \mathrm{~mm}$, with negligible shear-web and cover-skin thicknesses. Though not required for an estimate of stringer weight, the stringer pitch is important given they also serve as suction chambers. Empirical correlations given by Howe (2004) for stringer pitch correlate directly with the flange thickness; the pitch is estimated at around $0.5 \mathrm{~m}$ over the main wing, and $10 \mathrm{~cm}$ over the wing-tip fins. As the suction chambers are typically much wider, additional partitions are unnecessary. Furthermore, despite the low stringer pitch over the wingtip-fins, chamber losses are unlikely to pose any serious problems: there is freedom to vary chamber depth, whilst the suction flow rates are small. 


\subsubsection{Weight Breakdown}

A breakdown of the aircraft structural weight is provided in Tab. 6.10. The total weight is around $74.7 \mathrm{mt}$, with the wing weight being 15 times greater than that of the fuselage. As a fraction of MTOW, the structural weight is around $40 \%$, whilst the wing weight alone is $37 \%$.

Table 6.10: LFW structural weight breakdown

\begin{tabular}{lclc}
\hline \hline Component & Wing & Component & Cabin \\
\hline Flange $(\mathrm{kg})$ & 36,790 & Skin $(\mathrm{kg})$ & 1,921 \\
Stringers $(\mathrm{kg})$ & 19,866 & Stringers $(\mathrm{kg})$ & 480 \\
Shear webs $(\mathrm{kg})$ & 9,184 & Vertical bulkheads $(\mathrm{kg})$ & 922 \\
Ribs $(\mathrm{kg})$ & 3,899 & Cabin floor $(\mathrm{kg})$ & 794 \\
Control surface $(\mathrm{kg})$ & 108 & Insulation $(\mathrm{kg})$ & 276 \\
\hline Total $(\mathrm{kg})$ & 69,847 & Total $(\mathrm{kg})$ & 4,393 \\
\hline \hline
\end{tabular}

The aircraft weight buildup is presented in Tab. 6.11. The OEW constitutes 58\% MTOW, however this may be higher once aeroelastic effects (Weishaar and Ashley (1973)), and additional items such as rivets, screws, etc, have been accounted for. Therefore, the available weight is reserved for additional structural items. The fuel weight with reserves is $29.9 \mathrm{mt}$ (see Sec. 6.1.6), around 16\% MTOW

\begin{tabular}{lc} 
Table 6.11: LFW aircraft weight build \\
\hline \hline Component & Weight $(\mathrm{kg})$ \\
\hline MTOW & 187,000 \\
Available weight & 26,351 \\
Design payload & 22,000 \\
Fuel with reserves & 29,534 \\
OEW & 109,112 \\
Structures & 74,240 \\
Landing gear & 7,480 \\
Fixed equipment & 22,000 \\
Propulsion & 4,500 \\
Suction pumps & 895 \\
\hline \hline
\end{tabular}

\subsubsection{Centre-of-Gravity Buildup and Landing Gear Arrangement}

Whilst the aircraft is on the ground, a number of important design criteria must be adhered to. These are detailed by Raymer (1999). The first concerns the location of the main landing 


\section{CHAPTER 6. CONCEPTUAL DESIGN STUDY}

gear and hence the elevation of the aircraft, i.e. how high the centre of gravity is above ground level, and the need to avoid wingtip contact with the ground during takeoff or landing. A second requirement concerns the load bearing capacity of the nose wheel. Finally, when taxiing around a sharp corner, the aircraft must not overturn. The key geometric parameters for the tricycle gear arrangement adopted in this study are shown in Fig. 6.30.

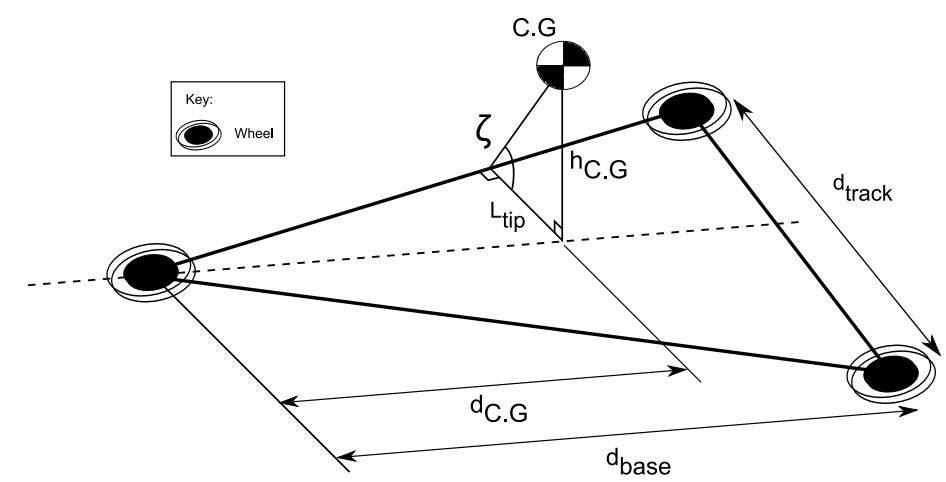

Figure 6.30: Tricycle landing gear geometric arrangement.

When the aircraft is at OEW, the C.G is at its maximum aft position of $11.9 \mathrm{~m}$. The maximum fore position is at $11.38 \mathrm{~m}$ when the aircraft is at MTOW. The main landing gear, positioned at $14 \mathrm{~m}$ from the nose, lies aft of the maximum rearwards C.G position; therefore, the aircraft is not in danger of tipping over whilst stationary.

To prevent "tipback" on takeoff, the angle between the main gear, C.G and the vertical, $\beta$, should be at least $15^{\circ}$. The calculated maximum takeoff rotation in Sec. 6.1.2.3 is 11.3'; therefore, a value for $\alpha$, at which the tips touch the ground, of $12^{\circ}$ is assumed. From Fig. 6.31 we can deduce that

$$
h_{C . G .}<=\frac{d}{\tan (\alpha+\beta)} .
$$

For $d=2.62 \mathrm{~m}$, this gives a maximum centre of gravity height of $5.14 \mathrm{~m}$. With a wing thickness of $2.5 \mathrm{~m}$, this translates, approximately, to a maximum landing gear height of 3.89 $\mathrm{m}$. With current airport infrastructures, boarding finger floors are positioned at a height of 5 $\mathrm{m}$. Therefore, a landing gear height of $3.75 \mathrm{~m}$ can meet this requirement whilst also satisfying tipback considerations.

The nose force borne by the wheel must not exceed $20 \%$ of the aircraft weight, but should be greater than $5 \%$ to ensure adequate wheel traction for taxiing. Vertical and moment equilibrium about the leading edge gives the proportion of weight $k_{n o s e}$ reacted by the nose 


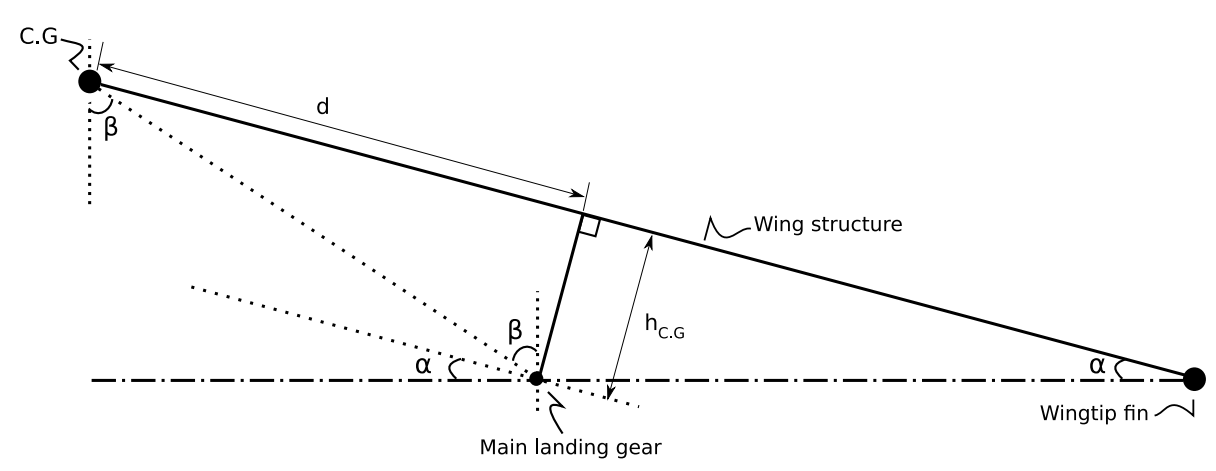

Figure 6.31: Tipback geometry.

wheel:

$$
k_{n o s e}=1-\frac{d_{C . G}}{d_{\text {base }}} .
$$

For $k_{\text {nose }}$ to be reasonably small, $d_{C . G}$ needs to be around the same size as $d_{b a s e}$, i.e. the C.G needs to lie close to the main landing gear. With the nose wheel at the apex of the aircraft, it bears between $15-19 \%$ of the aircraft's weight.

The overturn angle $\zeta$, as illustrated in Fig. 6.30, is the angle from the C.G to the main wheel looking from the main wheel to the nose wheel, at a location where the main wheel is aligned with the nose wheel. On consideration of the geometric parameters detailed in Fig. 6.32, it can be shown that

$$
\zeta=\arctan \left(\frac{h_{C . G} \sqrt{\left(d_{\text {track }} / 2\right)^{2}+\left(L+d_{C . G}\right)^{2}}}{d_{C . G} d_{\text {track }} / 2}\right) .
$$

Raymer (1999) gives a range for $\zeta$ between $50^{\circ}-60^{\circ}$, below which an aircraft has a reduced tendency to overturn. With a wheel track of $30 \mathrm{~m}$, at the maximum aft C.G position $\zeta=$ $17.5^{\circ}$, which is comfortably in the safe region. 


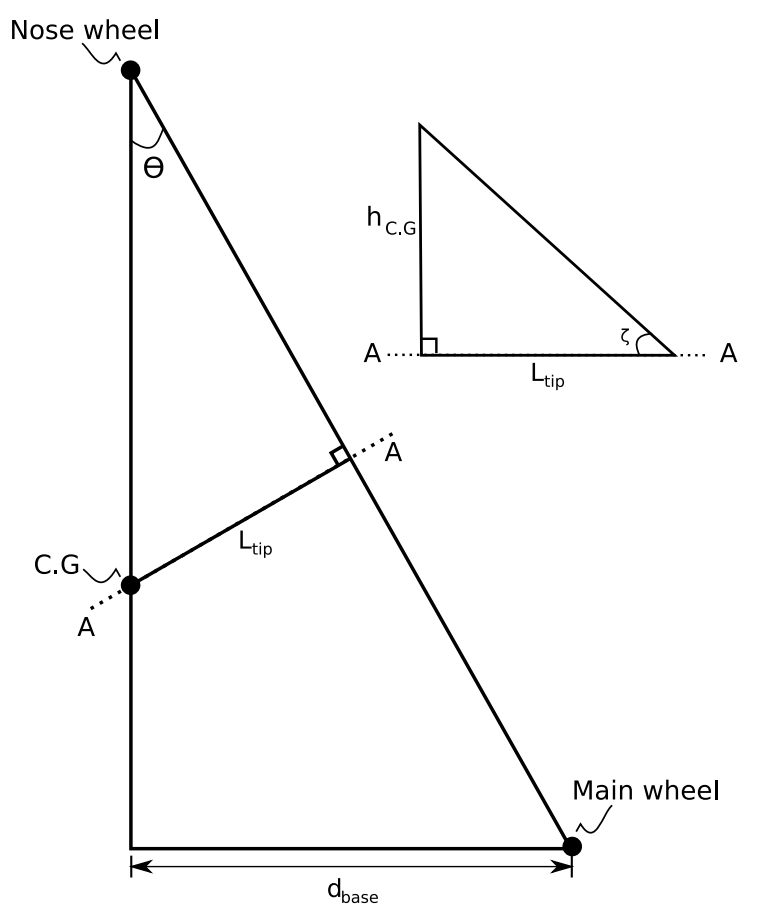

Figure 6.32: Overturn geometry.

\subsubsection{Mission}

The mission, reserve and unusable fuel weights are calculated following the methods outlined in Chap. 3. The fuel weight breakdown is summarised in Tab. 6.12. For a range of $9000 \mathrm{~km}$, the mission fuel weight is $27.4 \mathrm{mt}$, of which $1.8 \mathrm{mt}$ (1\% MTOW) is required to provide the takeoff and climb-out potential and kinetic energy gains. Combined with a passenger-payload of 220 pax, the estimated fuel burn is $13.9 \mathrm{~g} /$ pax.km.

Table 6.12: LFW mission-fuel weight buildup

\begin{tabular}{lc}
\hline \hline Component & Weight $(\mathrm{kg})$ \\
\hline Total & 29,534 \\
Mission & 27,434 \\
Reserve & 1,808 \\
Unusable & 292 \\
\hline \hline
\end{tabular}




\subsection{Competitor Aircraft Design Concept}

In this section, a conventional turboprop aircraft is designed (following the methodology outlined in Chap. 3) for the same mission specification as the LFW (passenger-payload of 220 persons, range of $9000 \mathrm{~km}$, and cruise Mach number of 0.67 ). This allows the performance benefits associated with the adoption of a flying-wing configuration and the utilisation of laminar flow control to be quantified directly.

With a similar passenger-payload and range, the Boeing 757-200 serves as an ideal donor. Where necessary, representative dimensions, areas, weights, and loadings are obtained from this aircraft ${ }^{1}$.

\subsubsection{Target Gross Weight}

In a single class cabin arrangement, the B757-200 has a passenger-payload of 228 persons and an MTOW of 115,680 kg. Scaling the donor aircraft to a passenger-payload of 220 persons, the target MTOW for the competitor aircraft is 111,621 kg. Hileman et al. (2007) assume that $2 \%$ MTOW fuel is burned by conventional aircraft in climb. This puts the estimated start of cruise weight at $109,388 \mathrm{~kg}$.

\subsubsection{Cruise Lift Coefficient}

The lift coefficient is given by

$$
C_{L}=\beta_{L F W} \sqrt{C_{D 0} \pi A_{R} e}
$$

For a fair comparison against the LFW, both aircraft must have the same propulsion characteristics, i.e., $\beta_{L F W}=0.736$. It is initially assumed that an Oswald efficiency, $e$, of 0.85 is achievable (Torenbeek (1976)). It therefore remains to evaluate $A_{R}$ and $C_{D 0}$.

A lower flight Mach number permits a reduced wing sweep angle relative to high-subsonic aircraft. Wing weight correlations provided by Raymer (1999) for transport aircraft show that, at some hypothetical wing weight, wing sweep may be traded for aspect ratio

$$
A_{R} \propto \frac{1}{\sqrt{\cos \Lambda_{1 / 4}}} .
$$

The B757-200 has a wing aspect ratio of 7.8 and quarter-chord sweep of $25^{\circ}$; unsweeping the wing gives a $22 \%$ increase in aspect ratio to a value of 9.5.

\footnotetext{
${ }^{1}$ www.aerospaceweb.org, November 2011
} 


\section{CHAPTER 6. CONCEPTUAL DESIGN STUDY}

van Es (2002) provides a method for estimating the zero-lift drag coefficient based on the principle of equivalent skin friction, which are linked via Eq. 6.16. The equivalent skinfriction coefficient is related to a Reynolds number, which depends on the reference length $S_{w e t} / b$, via an empirical correlation given by Eq. 6.17. A first estimate of the wetted area is given by assuming a value for $S_{w e t} / S_{r e f}$ of 5.6 , and a wing loading equal to that of the B757200 estimated at $6003 \mathrm{~N} / \mathrm{m}^{2}$ (this also helps in achieving satisfactory takeoff and landing performance). Evaluation of the zero-lift drag coefficient is iterative as it depends on altitude and velocity. The final, converged, value is 0.0166 . This compares well with the typical range for high-subsonic-speed jets, provided by Torenbeek (1976), of $0.014-0.020$.

$$
C_{D 0}=C_{f e}\left(S_{w e t} / S_{r e f}\right)
$$

with

$$
C_{f e}=0.00258+0.00102 \exp \left(-6.28 \times 10^{-9} R e\right)+0.00295 \exp \left(-2.01 \times 10^{-8} R e\right)
$$

and

$$
R e=\left(S_{w e t} / b\right) V / \nu
$$

The final estimate of $C_{L}$ is 0.48, compared to around 0.58 for the B757-200. Furthermore, the cruise altitude is $11,450 \mathrm{ft}$ lower at $23,550 \mathrm{ft}$. To maintain a constant $C_{L}$ and $M_{\infty}$ between start and end of cruise, the aircraft must climb to $30,928 \mathrm{ft}$, whilst the cruise speed must drop from $207 \mathrm{~m} / \mathrm{s}$ to $202 \mathrm{~m} / \mathrm{s}$ (assuming end of cruise weight is equal to OEW plus payload see Sec. 6.2.6 for further details of the aircraft weight breakdown). With a reduction of 9\% in unit Reynolds number, the change in skin-friction coefficient (see Chap.3) for a turbulent boundary layer along the wing is less than $2 \%$; therefore, the expected change in $L / D$ is negligible.

\subsubsection{Aircraft Geometry}

In this section, the methods used to define the fuselage, wing, vertical fin and horizontal tailplane are summarised. Figure 6.34 shows the aircraft geometry in plan, side and front view. Table 6.13 details key geometric parameters.

\subsubsection{Fuselage}

A narrow-body aircraft is desired. With a single-aisle cabin, and six passengers per row, the fuselage width is $3.55 \mathrm{~m}$ (based on the value of $0.592 \mathrm{~m}$ per passenger introduced in Sec. 6.1.1). With a $1 \mathrm{~m}$ seat pitch (as assumed for the LFW), and galleys, toilets, wardrobes, 
etc, which require a floor area of around $7 \mathrm{~m}^{2}$ (as assumed in Sec. 6.1.1.3), the minimum cabin length is $38.7 \mathrm{~m}$. The fuselage length is scaled with the cabin length of the B757-200. For simplicity, the fuselage is modelled as a cylinder with a tapered tail and elliptic nose.

\subsubsection{Wing}

Structural efficiency is compromised for better aerodynamic efficiency with a wing-taper ratio of 0.50. The wing quarter-chord is positioned at $48 \%$ of the fuselage length. (In Sec. 6.2.4 it is shown that this gives satisfactory balance and stability characteristics.) A high-mounted wing is selected to accommodate the large propeller-blade-diameter turboprops (see Sec. 6.2.5). A RAE2822 aerofoil section with a maximum thickness-to-chord ratio of 0.12 is chosen for the unswept wing, as it has been shown to exhibit satisfactory aerodynamic characteristics at transonic cruise Mach numbers (Drela and Giles (1987)).

\subsubsection{Tailplane}

Figure 6.33 is used to determine the vertical fin height. It shows that the stabiliser must lie outside regions of influence of the main wing when stalled. A T-tail plane is adopted to avoid the interaction of the engine efflux with the horizontal stabiliser. For the assumed wing position, a fin height of $8.7 \mathrm{~m}$ is required.

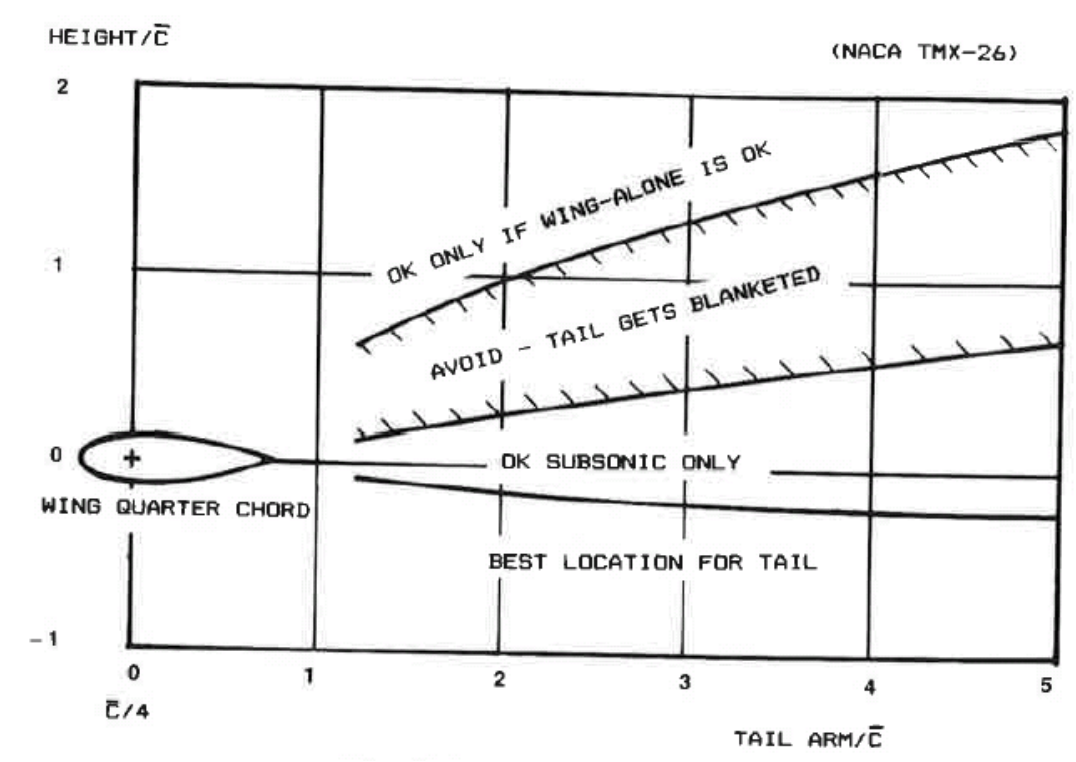

Figure 6.33: Aft tail positioning (from Raymer (1999)). 


\section{CHAPTER 6. CONCEPTUAL DESIGN STUDY}

The surface areas are determined using an empirical correlation given by Raymer (1999). That for the horizontal tailplane is given by

$$
S_{H T}=c_{H T} c S_{r e f} / L_{H T},
$$

where the horizontal tailplane volume coefficient has a value $c_{H T}$ of $1.0 ; L_{H T}$ is the distance from the wing and stabiliser quarter chords - Raymer (1999) indicates that this is roughly equal to $50 \%$ of the fuselage length. Similarly, the vertical fin area is given by

$$
S_{V T}=c_{V T} b S_{r e f} / L_{V T},
$$

where $c_{V T}=0.09$ (accounting for end-plate effects associated with a T-tail arrangement), and $L_{V T} \simeq L_{H T}$. A typical taper ratio of 0.60 is selected for the vertical fin. Sweep of the vertical fin is not essential here given the low cruise Mach number; however, the fin has a leadingedge sweep of $9^{\circ}$ due to the combination of a straight trailing edge and fin taper. In contrast, the stabiliser is untapered with the chord length chosen to match the fin-tip chord length. A sweep of $5^{\circ}$ is chosen on the recommendation of Raymer (1999) to avoid the stabiliser stalling simultaneously with the main wing at high angles of attack. Symmetrical NACA4-digit aerofoils are selected for both surfaces. For satisfactory structural performance, the maximum thickness-to-chord ratio is $10 \%$.

Table 6.13: Competitor aircraft geometric parameters.

\begin{tabular}{lc}
\hline \hline Parameter & Value \\
\hline Wing area $\left(\mathrm{m}^{2}\right)$ & 178.75 \\
Wing span $(\mathrm{m})$ & 41.2 \\
Wing $A_{R}$ & 9.5 \\
Mean wing chord $(\mathrm{m})$ & 4.3 \\
Fin area $\left(\mathrm{m}^{2}\right)$ & 29.3 \\
Stabiliser height $(\mathrm{m})$ & 8.7 \\
Stabiliser area $\left(\mathrm{m}^{2}\right)$ & 25 \\
Stabiliser span $(\mathrm{m})$ & 13.55 \\
Stabiliser $A_{R}$ & 6.3 \\
\hline \hline
\end{tabular}




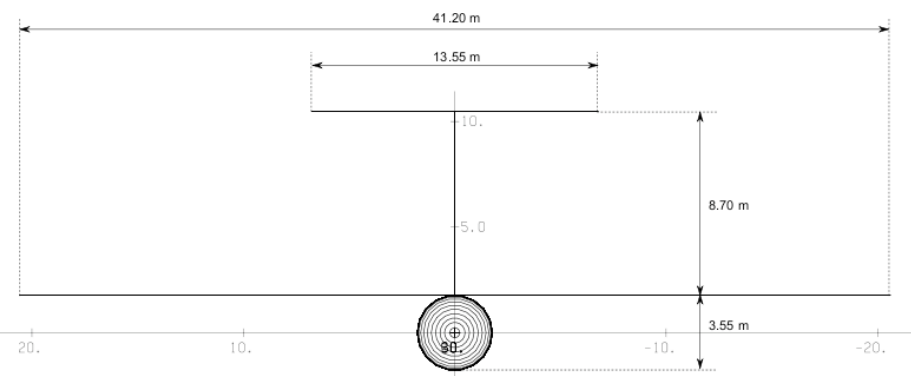

(a) Front view.

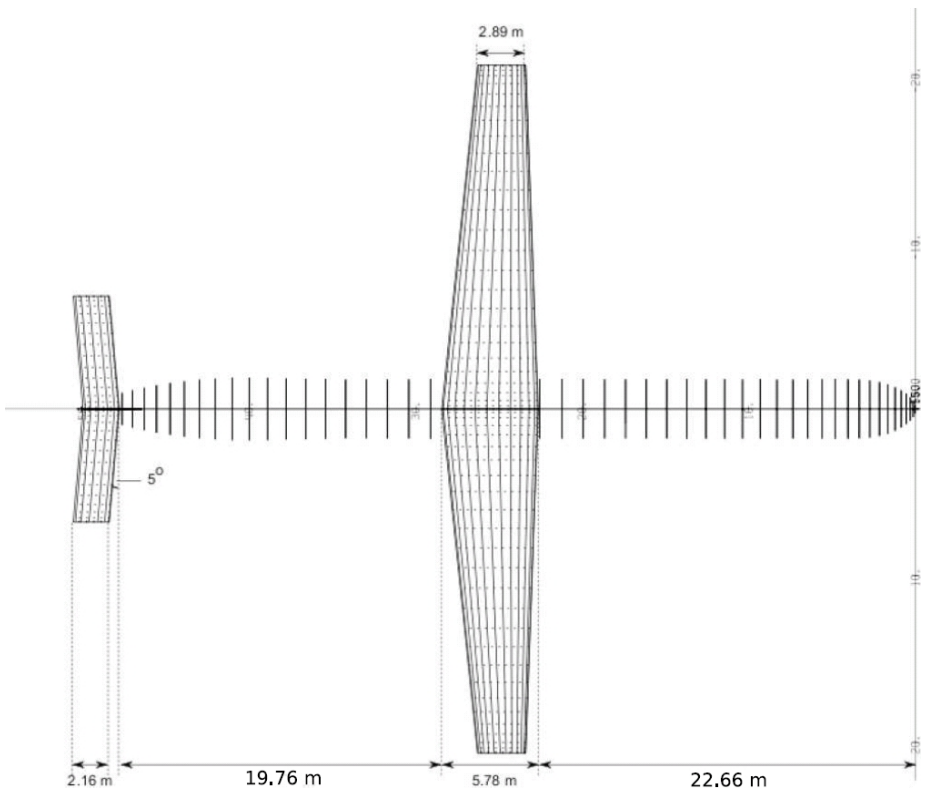

(b) Plan view.

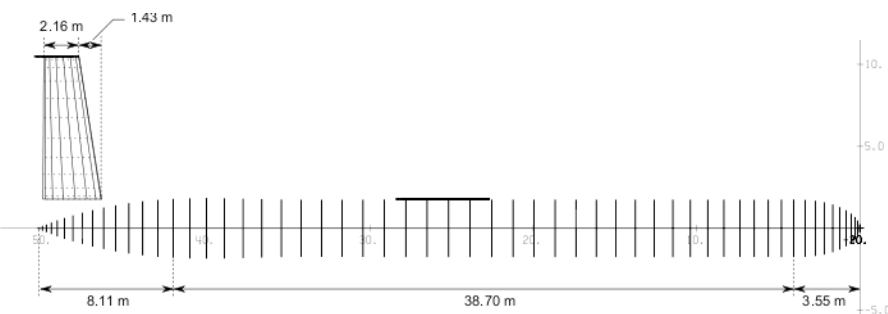

(c) Side view.

Figure 6.34: Competitor aircraft configuration. 


\subsubsection{Aircraft Performance}

In assessing the aerodynamic performance, the cruise and low-speed flight conditions are studied (with more emphasis on the former).

\subsubsection{Cruise Performance}

The longitudinal NP is $25.4 \mathrm{~m}$ from the nose. With the C.G. initially unknown, a $5 \%$ static margin is assumed, which puts its location at $24.1 \mathrm{~m}$ from the nose. (On evaluation of C.G. travel in Sec. 6.2.6, the final estimate does not warrant further iteration.) Pitch trim is achieved with a stabiliser incidence of $-1.05^{\circ}$ and zero elevator deflection. With no wing twist, the fuselage incidence is $2.1^{\circ}$, which is $0.9^{\circ}$ below the recommended maximum.

Figure 6.35 details the spanwise loading and $C_{l}$ distributions. With a high-mounted wing configuration, the lift distribution is not significantly affected by the presence of the fuselage. The lift coefficient increases outboard due to wing taper. The increased spanwise loading, $C_{l} C / C_{r e f}$, going inboard is structurally favourable. The local $C_{l}$ on the tailplane is negative, corresponding to the download required for pitch trim. The Oswald efficiency is $0.98: 15 \%$ better than that assumed in Sec. 6.2.2.

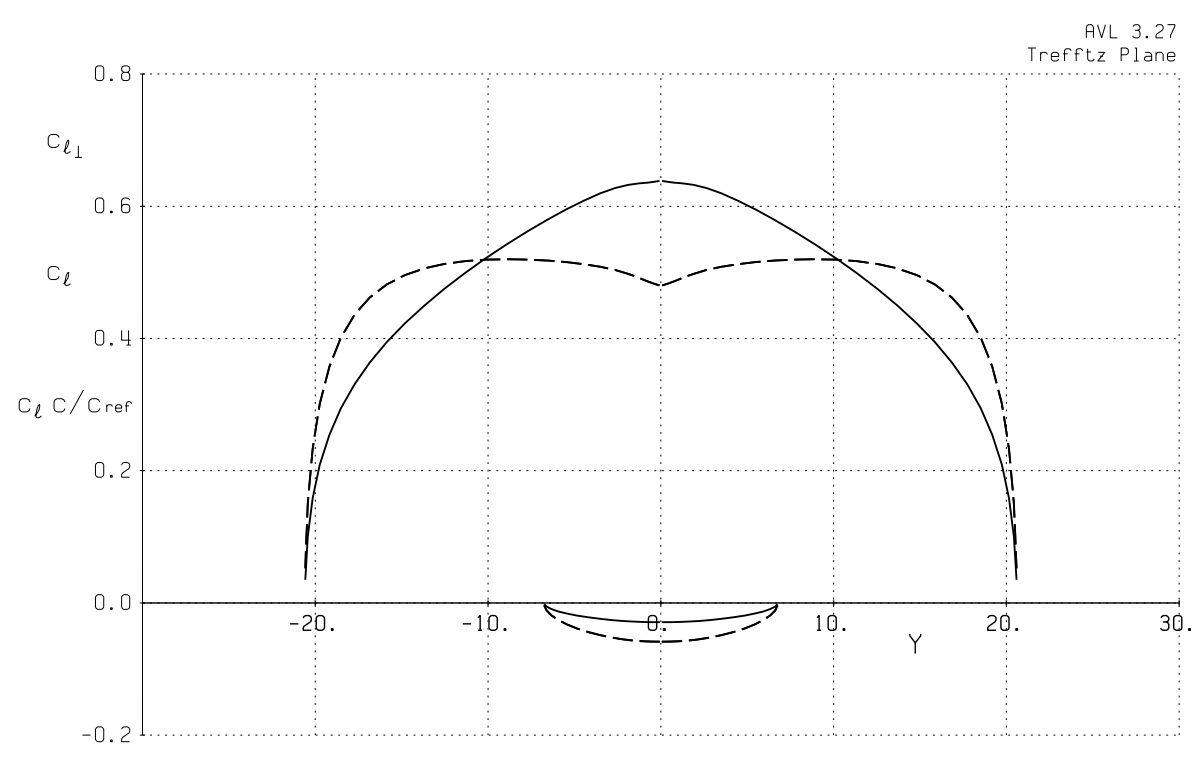

Figure 6.35: Spanwise loading distributions for $C_{L}=0.48$ and $M_{\infty}=0.67-C_{l}$ (dashed) and $C_{l} c / c_{r e f}$ (solid). 
Figure 6.36 shows a cross section of the RAE2822 aerofoil and surface $C_{p}$ distribution at the maximum calculated $C_{l}$ and Mach number. The critical pressure coefficient $C_{p}^{*}$ is superposed, and reveals that the flow remains subcritical throughout. Example surface $C_{p}$ distributions at the $C_{l}$ s corresponding to the stabiliser can be found in Chap. 4 .

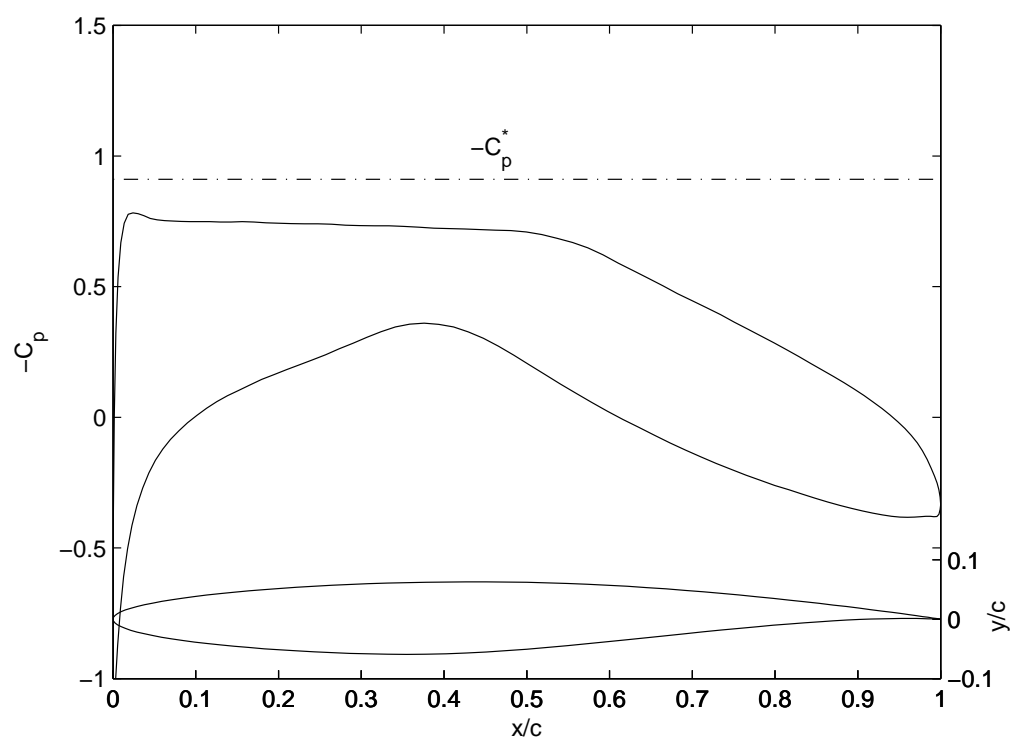

Figure 6.36: Surface $C_{p}$ distribution for an RAE2822 aerfoil, with the critical $C_{p}$ superposed $-C_{l}=0.52$ and $M_{\infty}=0.67$.

The aeroplane profile drag in the en-route configuration is evaluated following the methods detailed in Chap. 3. A breakdown is provided in Tab. 6.14. The estimated lift-to-drag ratio is 19.3, which is at the upper end of typical conventional configuration values (Cumpsty (1997)).

\subsubsection{Low-Speed Performance}

Torenbeek (1976) provides a useful first-order approximation to the drag polar on initial climb-out after take-off

$$
C_{D_{V_{2}}}=C_{0}+\frac{C_{L_{V_{2}}}^{2}}{\pi A_{R} E}
$$

For a takeoff speed $V_{2}$ of $70 \mathrm{~m} / \mathrm{s}, C_{L_{V_{2}}}=2.04 . \quad E$, with flaps extended and one engine inoperative, is taken as 0.672 , and $C_{0}=0.018$; evaluating Eq. 6.21, $C_{D_{V_{2}}}=0.225$. The lift-to-drag ratio is therefore around 9 . 
Table 6.14: Competitor aircraft cruise lift and drag coefficient breakdown.

\begin{tabular}{lc}
\hline \hline Coefficient & Value \\
\hline$C_{L}$ & 0.48 \\
$C_{D}$ & 0.0249 \\
$C_{D, i}$ & 0.0072 \\
$C_{D}$ wing & 0.0066 \\
$C_{D}$ vertical fin & 0.0011 \\
$C_{D}$ stabiliser & 0.0012 \\
$C_{D}$ fuselage & 0.0068 \\
$C_{D}$ engine nacelles & 0.0014 \\
$C_{D}$ interference effects & 0.0002 \\
$C_{D}$ protuberances, surface imperfections, etc & 0.0004 \\
\hline \hline
\end{tabular}

\subsubsection{Propulsion System}

The engine design approach detailed in Chap. 3 is followed. As top-of-climb is generally taken to be the design point for conventional aircraft, this is assumed here without further justification. Note, a design burner exit temperature of $1600 \mathrm{~K}$ is assumed.

Two turboprop engines sized to meet conditions at top-of-climb are unable to meet takeoff thrust requirements without encountering a high thrust coefficient. Therefore, a four engine configuration is adopted. Takeoff thrust is based on a minimum climb-out angle of $1.72^{\circ}$ with one engine inoperative (following Mair and Birdsall (1992)); the top-of-climb and cruise angles are $0.5^{\circ}$ and $0^{\circ}$, respectively (as in Sec. 6.1.4). The requirements per engine are summarised in Tab. 6.15; in addition, there is an auxiliary power off-take of $50 \mathrm{~kW}$. To avoid problems with high thrust coefficient values, a rotational speed of 1100 RPM and blade diameter of $4.3 \mathrm{~m}$ are selected.

Table 6.15: Competitor aircraft engine design variables.

\begin{tabular}{lcccc}
\hline \hline Description & T.O.C. & Start of Cr. & End of Cr. & T/O \\
\hline Altitude $(\mathrm{m})$ & 7178 & 7178 & 9427 & 120 \\
Mach number & 0.67 & 0.67 & 0.67 & 0.21 \\
Thrust $(\mathrm{kN})$ & 16.2 & 13.9 & 10.1 & 51.3 \\
\hline
\end{tabular}

A summary of the engine performance at top-of-climb, start and end of cruise, and takeoff, is provided in Tab. 6.16. The engine has satisfactory values for compressor and burner exit temperature over the range of operating conditions. The maximum compressor ratio occurs at top-of-climb and has a value of 21.4, which, as explained in Sec. 6.1.4, is within the capabilities of modern turboprop engines. The engine operates well away from the surge/stall line, with surge margins between $20-30 \%$. The range of values of advance ratio, thrust and power 
coefficients are typical of current turboprop blades. The propeller efficiency is maximised for cruise with a value of around $90 \%$. Overall efficiency is maximum at top-of-climb, but is of a similar order at start of cruise. The average overall efficiency in cruise is around $35 \%$. The maximum shaft power occurs at takeoff, and has a value of $5345 \mathrm{~kW}$. Scaling the dry engine weight based on this parameter (see Sec. 6.1.4), the weight per engine is $1.26 \mathrm{mt}$. Finally, the thrust specific fuel consumption for cruise has an average value of $13.6 \mathrm{~g} / \mathrm{kN} . \mathrm{s}$.

Table 6.16: Competitor aircraft engine performance summary.

\begin{tabular}{lcccc}
\hline \hline Parameter & T.O.C. & Start of Cr. & End of Cr. & T/O \\
\hline Compressor exit temperature (K) & 700 & 677 & 634 & 719 \\
Burner exit temperature (K) & 1600 & 1534 & 1472 & 1605 \\
Compressor pressure ratio & 21.4 & 19.4 & 19.4 & 17.9 \\
Surge margin (\%) & 22.5 & 25.7 & 24.7 & 29.4 \\
Advance ratio & 2.62 & 2.62 & 2.54 & 0.90 \\
Thrust coefficient & 0.247 & 0.214 & 0.202 & 0.354 \\
Power coefficient & 0.723 & 0.622 & 0.572 & 0.465 \\
Propeller efficiency & 0.897 & 0.901 & 0.900 & 0.682 \\
Shaft power delivered & 3964 & 3414 & 2403 & 5345 \\
Propulsive efficiency & 0.885 & 0.885 & 0.885 & 0.683 \\
Thermal efficiency & 0.418 & 0.412 & 0.412 & 0.359 \\
Overall efficiency & 0.358 & 0.352 & 0.355 & 0.245 \\
Thrust spec. fuel cons. (g/kN.s) & 13.56 & 13.81 & 13.40 & 6.74 \\
\hline \hline
\end{tabular}

\subsubsection{Structures and Weights}

\subsubsection{Structural Weight}

The aircraft structural weight is determined following the sizing methods outlined in Chap. 3 . As for the LFW, the construction material is aluminium 2024-T3.

A 2.5-g pull-up manoeuvre in cruise flight is taken as the critical loading condition for the wing. System loads acting on the wing half-span consist of two engines mounted on the leading edge at $24 \%$ and $66 \%$ half-span. (In Sec. 6.2 .5 the weight per engine is estimated at $1.26 \mathrm{mt}$.) The main landing gear is attached to the fuselage at $57 \%$ of its length. Fuel weight acts to provide bending relief, and is therefore not included in the calculation. The total estimated wing structural weight is approximately $15.3 \mathrm{mt}$, which is around 14\% MTOW; a breakdown is provided in Tab. 6.17 .

Howe (2004) notes that, typically, a pull-out manoeuvre in a nose dive is critical for the horizontal stabiliser; here, the lift distribution on the tailplane in trimmed cruise is scaled to a maximum section $C_{l}$ of 0.80 (Polentz (1949)). The vertical fin is sized to react loads 


\section{CHAPTER 6. CONCEPTUAL DESIGN STUDY}

Table 6.17: Competitor aircraft wing structural weight buildup.

\begin{tabular}{lc}
\hline \hline Component & Mass $(\mathrm{kg})$ \\
\hline Skin \& stringers & 14,010 \\
Spar webs & 448 \\
Ribs & 370 \\
Flaps, slats, etc & 459 \\
\hline Total & 15,287 \\
\hline \hline
\end{tabular}

incurred during engine-out at takeoff and is scaled by a factor of 0.70 to account for inertial effects (Greitzer et al. (2010)). The total tailplane weight is $1.6 \mathrm{mt}$, which is less than $2 \%$ MTOW.

Table 6.18 shows a breakdown of the fuselage structural weight. The fuselage shell, tail cone, floor and added bending material are sized to withstand applied loadings which include: differential pressure, horizontal and vertical bending moments in flight and on landing, and torsional shear (Greitzer et al. (2010)). The remainder of the fuselage weights are estimated using empirical correlations provided by Torenbeek (1976) for: shell modifications, consisting of added weights including rivets, fasteners, local reinforcements, etc; and the support structures of the wing, tailplane and fuselage mounted landing gear. The total estimated fuselage weight is $10 \mathrm{mt}$, around $9 \%$ MTOW.

Table 6.18: Competitor aircraft fuselage structural weight breakdown.

\begin{tabular}{lc}
\hline \hline Component & Mass $(\mathrm{kg})$ \\
\hline Shell & 1,891 \\
Tail cone & 9 \\
Floor & 803 \\
Additional horizontal bending material & 46 \\
Windows & 509 \\
Insulation & 311 \\
Shell modifications & 1891 \\
Support structures & 4564 \\
\hline Total & 10,024 \\
\hline \hline
\end{tabular}

The total structural weight is estimated at around $26.9 \mathrm{mt}$. A breakdown is given in Tab. 6.19. For comparison, the wing, tail and body structures of the B737-200 comprise $43 \%, 11 \%$ and $46 \%$, respectively, of the total structural weight ${ }^{1}$.

\footnotetext{
${ }^{1}$ http://adg.stanford.edu/aa241/AircraftDesign.html, December 2011
} 
Table 6.19: Competitor aircraft structural weight breakdown.

\begin{tabular}{lc}
\hline \hline Component & Mass $(\mathrm{kg})$ \\
\hline Wing & 15,287 \\
Tailplane & 1597 \\
Fuselage & 10,024 \\
\hline Total & 26,908 \\
\hline \hline
\end{tabular}

\subsubsection{Fuel Weight}

The mission, reserve and unusable fuel weights are calculated following the methods outlined in Chap. 3. The fuel weight breakdown is summarised in Tab. 6.20. For a range of $9000 \mathrm{~km}$, the mission fuel required is $29.6 \mathrm{mt}$, of which $0.7 \mathrm{mt}(0.6 \% \mathrm{MTOW})$ is to raise the potential and kinetic energies between takeoff and cruise. With a passenger-payload of 220 persons, the estimated fuel burn is $15.0 \mathrm{~g} /$ pax.km.

Table 6.20: Competitor fuel weight breakdown.

\begin{tabular}{lc}
\hline \hline Fuel & Mass $(\mathrm{kg})$ \\
\hline Total & 30,942 \\
Mission & 29,608 \\
Reserve & 1,028 \\
Unusable & 306 \\
\hline \hline
\end{tabular}

\subsubsection{Aircraft Weight Buildup and Centre-of-Gravity Travel}

The aircraft weight buildup is presented in Tab. 6.21. The OEW contributes 52\% MTOW, which compares well with conventional aircraft configurations. For comparison, the Boeing 757-200 has an empty-weight fraction of 0.55 (Aboulafia (1996)). Fuel with reserves represents $28 \%$ MTOW.

The maximum aft C.G. location is at $25.1 \mathrm{~m}$ when the aircraft is loaded with fuel only. In contrast, the maximum fore location corresponds to the aircraft loaded with payload only at $23.9 \mathrm{~m}$. The mean C.G. position during cruise is $24.35 \mathrm{~m}$, which is approximately $2 \%$ further aft of that assumed for the pitch trim analysis in Sec. 6.2.4.1. Furthermore, there is no danger of tipback, with the landing located at $28.4 \mathrm{~m}$ (see Sec. 6.2.6.1). 
Table 6.21: Competitor aircraft weight buildup.

\begin{tabular}{lc}
\hline \hline Component & Mass $(\mathrm{kg})$ \\
\hline MTOW & 111,621 \\
OEW & 58,397 \\
Available weight & 282 \\
Design payload & 22,000 \\
Fuel with reserves & 30,942 \\
Structure & 26,908 \\
Fixed equipment & 22,000 \\
Landing gear & 4,465 \\
Propulsion & 5,024 \\
\hline \hline
\end{tabular}

\subsection{Comparison of LFW and Competitor Aircraft}

A comparison of the MTOW breakdown for each aircraft is detailed in Fig. 6.37. The LFW has a MTOW of $187 \mathrm{mt}$ relative to around $112 \mathrm{mt}$ for the competitor aircraft. With a $69 \%$ lower wing loading, the LFW has a significantly higher structural weight fraction of $40 \%$, compared to just $22 \%$ for the competitor. The LFW has an OEW which is $58 \%$ of MTOW, higher than that for the competitor at around $52 \%$. The LFW values may also increase on inclusion of aeroelastic effects and additional item weights; therefore, the available weight is reserved for this purpose, allowing the OEW potentially to increase to $72 \%$ of MTOW.

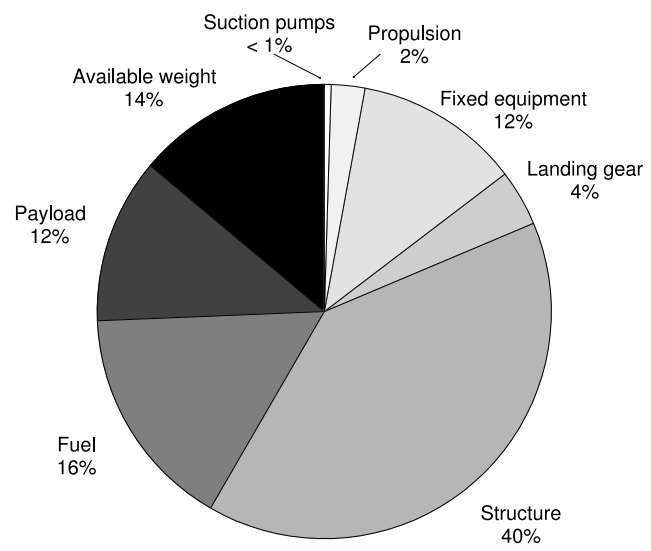

(a) LFW - MTOW of $187 \mathrm{mt}$.

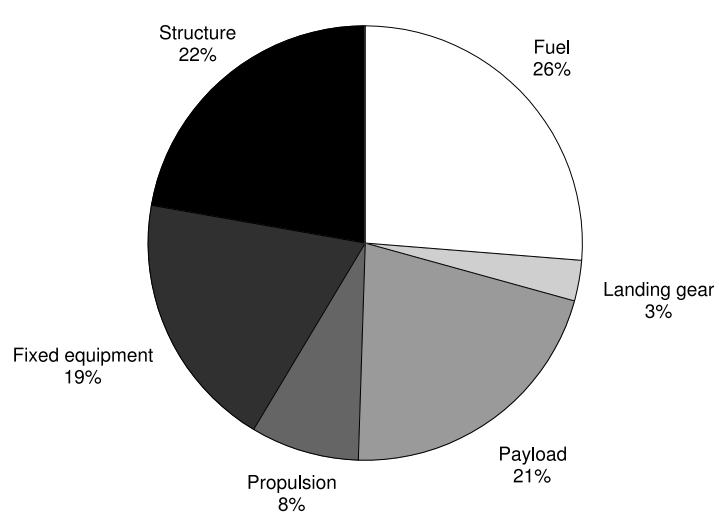

(b) Competitor aircraft - MTOW of $111.6 \mathrm{mt}$.

Figure 6.37: Comparison of LFW and competitor aircraft MTOW breakdowns.

The cruise lift-to-drag ratios of the LFW and competitor aircraft are 60.9 and 19.3, respectively. The value for the competitor aircraft is comparable to current advanced aircraft. 
For the LFW, an all-lifting surface with wingtip fins contributes to a $10 \%$ better Oswald efficiency, whilst the utilisation of suction over $90 \%$ of the wetted area leads to a $93 \%$ lower viscous drag coefficient.

However, the superior lift-to-drag ratio comes at a cost: the overall engine efficiency is significantly compromised, with a value of $21 \%$. In contrast, the competitor aircraft has an efficiency of $35 \%$. This corresponds to a lower competitor engine $s f c$ of $13.6 \mathrm{~g} / \mathrm{kN}$.s relative to $22.6 \mathrm{~g} / \mathrm{kN}$.s for the LFW. The poor performance of the LFW engine is due to the large difference in thrust requirement between takeoff and cruise. Despite a higher takeoff L/D of 22.4 relative to a value of 9 , the LFW has a takeoff thrust per engine comparable to the competitor aircraft, due to the aircraft weight contribution. As a consequence, the engine design point is at takeoff, which leads to the degraded performance in cruise. In contrast, the competitor aircraft engine is designed at top-of-climb, as is typical, and maximises efficiency in cruise.

Despite a lift-to-drag ratio that is over three times higher for the LFW, the higher $s f c$ and higher aircraft weight compromise fuel burn. The LFW has an estimated fuel burn of $13.9 \mathrm{~g} /$ pax.km, which is just $7 \%$ lower than the competitor aircraft at $15.0 \mathrm{~g} /$ pax.km.

\subsection{Summary}

This chapter has described the conceptual design for a laminar-flying-wing aircraft, based on the design specification presented in Chap. 5. It is found that a laminar flying wing with: satisfactory stability and control characteristics, accommodation for 220 pax at adequate passenger comfort levels, distributed suction over $90 \%$ of the wetted area for the maintenance of laminar flow, and powered by three turboprop engines, is capable of flying a distance of $9000 \mathrm{~km}$ at a Mach number of 0.67 with a fuel burn of $13.9 \mathrm{~g} /$ pax.km. This figure compares favourably with the value of $23.5 \mathrm{~g} /$ pax.km quoted for the Boeing 777 (Martinez-Val (2007)). However, it falls short of the $60 \%$ reduction suggested by Greener by Design, due to low values of engine efficiency and payload weight fraction.

To put the LFW estimate in context, a conventional, turbulent-flow, aircraft was designed for the same mission specification. A high wing configuration was selected to accommodate four, large diameter, turboprop engines, which in turn required a T-tailplane configuration. The reduced cruise Mach number permitted an unswept wing configuration, which led to a lift-to-drag ratio comparable to current advanced aircraft. In contrast to the LFW, the competitor aircraft's engines had favourably high efficiencies in cruise and the payload weight fraction was typical, leading, despite a lower lift-to-drag ratio, to a comparable fuel burn estimate just $7 \%$ higher at $15 \mathrm{~g} /$ pax.km. 
In summary, the exceptional aerodynamic efficiency of the laminar flying wing design presented here is associated with penalties in aircraft weight and propulsion efficiency, to the extent that a conventional turboprop aircraft flying at the same speed might be almost as efficient. Unless these penalties can be significantly mitigated, the LFW will fall short of the potential suggested by considering its lift-to-drag ratio in isolation. 


\section{CHAPTER 7}

\section{Conclusions And Future Work}

\subsection{Conclusions}

\subsubsection{Motivation for the Study}

Growing air travel and its environmental impact is increasingly becoming a public concern. The laminar flying wing, which utilises boundary-layer suction as a means of laminar flow control, has been proposed by Greener by Design as a potential solution. On the basis of the Breguet range equation, and estimates for various component parameters, Green (2002) proposes that a laminar flying wing, with 2001 technology levels and powered by UDF engines, would consume $60 \%$ less fuel relative to a conventional aircraft. It is necessary to go back 50 years, to the Handley Page HP117, to find a conceptual design that provides a quantitative fuel burn estimate. However, the estimated fuel burn is only marginally better than that of the B787, and therefore falls short of the gains expected of it. New studies, assuming present-day technology, are therefore required to substantiate (or challenge) the Greener by Design proposal. In this thesis, one such study has been produced.

\subsubsection{Conclusions of the Study}

I. The basic architecture of practical laminar flow control systems is well established. However, little guidance on the important design parameters and their interactions is available. This study has aimed to provide such insight for a commercial laminar-flyingwing transport aircraft.

It has identified a minimum power requirement, independent of the suction system design, associated with the boundary layer stagnation pressure loss of the flow in an optimal suction distribution. Furthermore, an automated algorithm has been developed to facilitate the design of a practical suction system, requiring only the specification 


\section{CHAPTER 7. CONCLUSIONS AND FUTURE WORK}

of the skin's surface resistance and the sensitivity of the suction distribution, to define the subsurface chamber width and pressure. Deviations from the optimal suction distribution have been found to have little effect on the overall suction coefficient, but additional power is required due to the skin's surface resistance. In the spanwise direction, through suitable choice of spanwise-length-to-chamber-depth, inertial and friction losses may be made negligibly small. Combined with the pressure drop from the aerofoil surface to the collector ducts, rather than to the chamber, which determines the power penalty, it is proposed that the number of collectors and (hence) pumps should equal the number of chambers. Furthermore, there should be few chambers, as increasing chamber architecture complexity does not reduce the power penalty. Finally, the study revealed that the additional power requirement associated with a practical suction-system implementation for a laminar flying wing is likely to consume at most $20 \%$, and more likely around $10 \%$, excess power above the minimum value.

II. The basic philosophy adopted was that the laminar flying wing would be designed with an emphasis, above all, on aerodynamic efficiency. A high-level exploration of the design space was performed to identify the key configuration and mission parameters, with the following constraints: wing sweep is restricted to $25^{\circ}$, to justify the neglect of additional suction requirements for the suppression of cross-flow instabilities; it must be possible to stand in the cabin; and the flow must remain subcritical. Furthermore, a configuration that could be analysed on the basis of simple methods was insisted upon. Therefore, the aircraft was treated as a swept, untapered, wing utilising distributed suction, without any regard for a practical suction-system implementation (on the basis of conclusion I.) Furthermore, the design was defined by three principal variables: thickness-to-chord ratio, unit Reynolds number, and span.

It was determined that optimum aerodynamic efficiency would be achieved by specifying the highest possible values for all three variables, which would also maximise wing loading, and hence minimise gust response issues. However, the combination of high thickness-to-chord ratio and unit Reynolds number was found to limit the degree of allowable sweepback due to attachment-line instability, hence raising the prospect of balance and stability issues, whilst high thickness-to-chord ratios also significantly compromised the cruise speed. Other potential problems identified included: the wing weight fraction, which was driven to higher values by the span length; unfavourably low cruise altitudes at higher unit Reynolds numbers; and passenger volume restrictions at higher thickness-to-chord ratios. These considerations drove the design towards a compromise specification: a 187 tonne, $80 \mathrm{~m}$ span, aircraft, with an aspect ratio of 6.4 , 


\subsection{CONCLUSIONS}

cruising at Mach 0.67 and an altitude of 22,500 ft, with lift coefficient 0.14 .

III. On the basis of this specification, a detailed conceptual design was performed. Five areas were assessed: 1) 3-D airframe generation, 2) aircraft performance, 3) suctionsystem technology integration, 4) propulsion system, and 5) structures and weights. In contrast to a conventional methodology, the approach for the laminar flying wing was essentially reversed, with the mission (i.e. range, Mach number, payload) being an output, once a viable aircraft specification had been identified. To quantify the benefits associated with the flying wing configuration utilising laminar flow control, the design was compared against a competitor, turbulent flow, aircraft with a conventional layout, designed for the same mission, the same propulsion characteristics, and following the same technical approach.

The key aspects of the laminar flying wing studied here are as follows:

- On consideration of passenger-roll comfort requirements, the outboard extent of the centrebody cabin limits the passenger capacity to 220 persons (i.e. a payloadweight fraction of $12 \%$ MTOW), and reduces the ( $80 \mathrm{~m}$ span) wing aspect ratio to 5.9 (from 6.4).

- Satisfactory stability and control characteristics are obtained with the utilisation of: wingtip fins, wing sweepback, symmetrical wing sections; but flight control systems may be required to artificially stabilise dynamic stability modes.

- Distributed suction over $90 \%$ of the wetted area significantly reduces the aircraft profile drag. When this is combined with a low induced drag (via a low cruise $C_{L}$, high-aspect-ratio wing, and a 'better than elliptic' lifting surface) the estimated cruise lift-to-drag ratio is 60.9 ; however, a practical suction-system implementation requires $1.87 \mathrm{MW}$ of power to drive the suction pumps.

- A low cruise Mach number enables the utilisation of more efficient turboprop, over turbofan, engines. However, the performance of both engine types is significantly degraded by the large difference in thrust requirement between takeoff and laminarised cruise. For this reason, and to avoid high thrust coefficients, three engines are required; furthermore, in contrast to conventional aircraft, the engine design point is taken to be at takeoff, which compromises the overall engine efficiency at $21 \%$, and engine $s f c$ at $22.6 \mathrm{~g} / \mathrm{kN} . \mathrm{s}$.

- The combination of a low wing loading, high-aspect-ratio wing, and the limit on outboard extent of the passenger payload, leads to a high structural weight of $40 \%$ MTOW, with an allowance to increase to $54 \%$ MTOW. 


\section{CHAPTER 7. CONCLUSIONS AND FUTURE WORK}

- On the basis of the mission fuel available, the range is $9000 \mathrm{~km}$.

The key aspects of the competitor aircraft are as follows:

- With a low cruise Mach number, wing sweep is traded for aspect ratio. The result is a zero-sweep wing with an aspect ratio of 9.5 and a span of $41.2 \mathrm{~m}$. The effect is a cruise lift-to-drag of 19.3, comparable to current advanced aircraft.

- Four large-blade-diameter turboprop engines are utilised to avoid excessive thrust coefficients at takeoff. The overall engine efficiency is $35 \%$, which results in an engine $s f c$ of $13.6 \mathrm{~g} / \mathrm{kN} . \mathrm{s}$.

- The MTOW is $112 \mathrm{mt}$, with a payload weight fraction of $20 \%$.

The laminar flying wing has an estimated fuel burn of $13.9 \mathrm{~g} /$ pax.km, whilst the competitor aircraft is just $7 \%$ higher at $15.0 \mathrm{~g} /$ pax.km. In conclusion: despite a superior lift-to-drag ratio, the high-aspect-ratio laminar flying wing proposed in this study suffers from a low payload-weight fraction and poor propulsion efficiency, and hence falls short of the potential expected of it by Greener by Design.

\subsection{Recommendations for Future Work}

If the high aspect ratio, low sweep and low wing loading aircraft presented in this study is to realise the substantial reduction in fuel efficiency anticipated by consideration of aerodynamic efficiency alone, the overall engine efficiency, which is compromised by the large difference in thrust requirement between takeoff and laminarised cruise, must be improved upon. This could either be through optimisation of the turboprop considered here, or by consideration of alternative, novel, design solutions. If this issue can be successfully addressed, then further design refinement would be required; considerations may include: an analysis of aeroelastic effects, which could lead to a higher structural weight than that calculated based on a static analysis alone; and design of the flight control system, in order to estimate the required control power.

If these issues cannot be overcome, then, on the basis of the design considerations presented in this study, relationships between the relevant parameters and their functional dependence on one another could be established to facilitate an optimisation study. The outcome of such a study may be the compromise of aerodynamic efficiency via a low-aspect-ratio wing. This configuration could potentially lead to an improvement in the overall engine efficiency, and reduce the structural weight fraction (whilst avoiding flutter issues). However, this would 


\subsection{RECOMMENDATIONS FOR FUTURE WORK}

demand an increased amount of wing sweepback, which would have the compounded benefit of a higher payload volume, but would increase the complexity of the aerodynamic analysis. 
CHAPTER 7. CONCLUSIONS AND FUTURE WORK 


\section{REFERENCES}

I.H. Abbott and A.E. Von Doenhoff. Theory of Wing Sections. Dover Publications Inc., 1959. 124,154

R. Aboulafia, editor. Jane's Civil Aircraft. Glasgow: Harper Collins Publishers, 1996. 85, 203

American Standard Atmosphere. TM-X 74335, NASA, 1976. 121

J.D. Anderson. A History of Aerodynamics. Cambridge University Press, 1997. 7

M.D. Ardema, M.C. Chambers, A.P. Patron, A.S. Hahn, H. Miura, and Moore. M.D. Analytical Fuselage and Wing Weight Estimation of Transport Aircraft. TM 110392, NASA, 1996. 25

A. Arnal, J. Perraud, and A. Seraudie. Attachment line and surface imperfection problems. In rto-avt/vki lecture series "advances in laminar-turbulent transition modelling", number RTO-EN-AVT-151, pages 15-1-15-22, 2008.

M. Ashby, H. Shercliff, and D. Cebon. Materials. Butterworth-Hienemann, 2001. 77

P.R. Ashill, R.F. Wood, and D.J. Weeks. An improved, semi-inverse version of the viscous Garabedian and Korn method (VGK). Tech. Rep. 87002, Royal Aircraft Establishment, 1987. 47

P.R. Ashill, J.L. Fulker, and R.F. Wood. On the Sectional Drag and Pressure Distributions of Swept-Wing Aerofoil Sections. TR 88073, Royal Aircraft Establishment, 1988. 51

C. Atkin. Performance trade-off studies for a retrofit Hybrid Laminar Flow Control system. 2nd AIAA Flow Control Conference, AIAA-2004-2215, 2004. 93, 97

S.A. Berry. Incompressible Boundary-Layer Stability Analysis of LFC Exp. Data for SubCritical Mach Numbers. CR 3999, NASA, 1989. 93 


\section{REFERENCES}

S.A. Berry, J. Dagenhart, C.W. Brooks, Jr., and C.D. Harris. Boundary Layer Stability Analysis of LaRC 8-foot LFC Experimental Data. N90-12532 N90-12532, NASA, 1987. 93

N.T. Birch. 2020 vision: the prospects for large civil aircraft propulsion. The Aeronautical Journal, 2000. xix, 2

A.L. Bolsunovsky, N. Buzoverya, B. Gurevich, V. Denisov, A. Dunaevsky, L. Shkadov, O. Sonin, A. Udzhuhu, and J. Zhurihn. Flying wing - problems and decisions. Pergamon, 2001. 3, 23, 24, 25, 28, 32, 37

A.H. Bowers and A.G. Sim. A comparison of Wortmann airfoil computer-generated lift and drag polars with flight and wind tunnel results. TM 86035, NASA, 1984. 92

A.L. Braslow. A History of Suction-Type Laminar-Flow Control with Emphasis on Flight Research. Monographs in Aerospace History 13, NASA, 1999. 17

D.M. Bushnell and M.R. Malik. Supersonic Laminar Flow Control. Technical Report N9012554, NASA, 1987. 93

M. Carlsson and J. Kuttnkeuler. Design and Testing of a Blended Wing Body Aeroelastic wind-tunnel model. Journal of Aircraft, 40(1), 2003.

M. Cook. Flight dynamics principles. Arnold Publishers, 1997. 35, 36, 146

N.A. Cumpsty. Jet Propulsion. Cambridge University Press, 1997. 57, 61, 141, 159, 170, 172, 199

R.M. Denning, J.E. Allen, and F.W. Armstrong. Future large aircraft design - the delta with suction. The Aeronautical Journal, 101(1005):187-198, 1997. xix, xx, 2, 7, 10, 12, 18, $38,39,42,123$

R.M. Denning, J.E. Allen, and F.W. Armstrong. The broad delta airliner. The Aeronautical Journal, 107(2784):547-558, 2003. 4

P.P. Doerffer and R. Bohning. Modelling of Perforated Plate Aerodynamics Performance. Aerosp. Sci. Technol., 4, 2000. 106, 107

C.J. Donlan. An Interim Report on the Stability and Control of Tailless Airplanes. Report 796, NACA, 1944. 27, 28, 29, 31, 32, 33, 123

M. Drela. XFOIL: An Analysis and Design System for Low Reynolds Number Airfoils. In T.J. Mueller, editor, Low Reynolds Number Aerodynamics Proceedings of the Conference, Lecture Notes in Engineering 54, Notre Dame, 1989. Springer. 49 
M. Drela and M.B. Giles. Viscous-Inviscid Analysis of Transonic and Low Reynolds Number Airfoils. AIAA Journal, 25(10), 1987. 154, 195

J.P. Eastwood. Green Aircraft: Wing Design. Master's thesis, University of Cambridge, 2008. 154

B. Edwards. Laminar Flow Control - Concepts, Experiences, Speculations. In Special Course on Drag Reduction, number AGARD-R-654, 1977. xix, 19, 20

J.B. Edwards. Boundary Layer and Flow Control, volume 2, chapter Fundamental Aspects of Propulsion for Laminar Flow Aircraft, pages 1077-1122. Pergamon Press, 1961. 10, 20, 21,97

J.E. Ellis, S.A. Walsh, and D.I.A. Poll. Assessment of the eN Method as a Transition Prediction Tool for Zero Pressure Gradient Flows with and without Boundary Layer Suction. In P. Thiede, editor, Aerodynamic Drag Reduction Technologies, Notes on Numerical Fluid Mechanics, volume 76, 2000. 92

R. Eppler. Turbulent Airfoils for General Aviation. Journal of Aircraft, 15(2), 1978. 92

R. Eppler and D.M. Somers. A computer program for the design and analysis of low-speed airfoils. TM 80210, NASA, 1980. 47, 50, 92, 95, 118

ESDU 96028. VGK method for two-dimensional aerofoil section - Part 1: Principles and Results., 2004. 50

F.J.J.M.M. Geuskens, S. Koussios, O.K. Bergsma, and A. Beukers. Non-Cylindrical Pressure Fuselages for Future Aircraft. 49th AIAA/ASME/ASCE/AHS/ASC Structures, Structural Dynamics, and Materials Conference, (AIAA-2008-1907), 2008. 183

J.E. Green. Greener by Design: The Technology Challenge. The Aeronautical Journal, 106 (1056):57-113, 2002. 1, 2, 3, 4, 37, 38, 42, 207

J.E. Green. Civil aviation and the environment - the next frontier for the aerodynamicist. The Aeronautical Journal, 110(1110):469 - 486, 2006. xix, xx, xxvii, 4, 8, 9, 10, 11, 12, 14, 20,40

N. Gregory. Boundary Layer and Flow Control, volume 2, chapter Research on Suction Surfaces for Laminar Flow, pages 924-960. Pergamon Press, 1961. 19, 38, 104, 106 


\section{REFERENCES}

E.M. Greitzer, P.A. Bonnefoy, E. De la Rosa Blanco, C.S. Dorbian, M. Drela, D.K. Hall, and et al. N+3 Aircraft Concept Designs and Trade Studies: Final Report/Appendix. CR 2010-216794/Vol.1/Vol.2, NASA, 2010. xix, xx, 2, 25, 48, 50, 51, 65, 68, 72, 73, 87, 202

P. Hall, M.R. Malik, and D.I.A. Poll. On the Stability of an Infinite Swept Attachment Line Boundary Layer. Proc. R. Soc. Lond., (A-1984-395):229-245, 1984. 14

C.D. Harris, W.D. Harvey, and C.W. Brooks, Jr. The NASA Langley Laminar-Flow-Control Experiment on a Swept, Supercritical Airfoil. TP 2809, NASA, 1988. xix, 13, 17, 93

M.R. Head. The Boundary Layer with Distributed Suction. Technical Report R. \& M. No. 2783, A.R.C., 1955. xix, 18, 19, 97

M.R. Head. Approximate Calculations of the Laminar Boundary Layer with Suction, with Particular Reference to the Suction Requirements for Boundary Layer Stability on Aerofoils of Different Thickness/Chord Ratios. Tech. Rep. R. \& M. No. 3124, A.R.C., 1959. 91, 94, 95

J.I. Hileman, Z.S. Spakovsky, M. Drela, M.A. Sargeant, and A. Jones. Airframe Design for Silent Fuel-Efficient Aircraft. Journal of Aircraft, 47(3), 2007. 67, 90, 126, 159, 193

E.L. Houghton and P.W. Carpenter. Aerodynamics for Engineering Students. Edward Arnold, fourth edition, 1993. 48, 126

D. Howe. Aircraft Loading and Structural Layout. Professional Engineering Publishing Ltd., 2004. xx, 65, 71, 72, 74, 76, 79, 81, 87, 184, 188, 201

G.R. Inger and H. Babinsky. Viscous Compressible Flow Across a Hole in a Flat Plate. Journal of Aircraft, 37(6), 2000. 107, 184

L.R. Jenkinson, P. Simpkin, and D. Rhodes. Civil Jet Aircraft Design, pages 30-31. Arnold Publishers, 1999. 65, 134, 139

R.T. Jones. Notes on the Stability and Control of Tailless Airplanes. Report 837, NACA, 1941. $28,29,32,33,34,123$

R.D. Joslin. Overview of Laminar Flow Control. Technical report, NASA, 1998a. xix, 7, 13, 15,18

R.D. Joslin. Aircraft Laminar Flow Control. Annu. Rev. Fluid Mech., 30(1-29), 1998b. xix, $14,16,17,92,93,124$ 
J. Katz and A. Plotkin. Low-Speed Aerodynamics. Cambridge University Press, second edition, 2001. 125

R.E. Kosin. Laminar flow control by suction as applied to the X-21A airplane. Journal of Aircraft, 2(5), 1965. xix, 21, 22

A.M. Kuethe and C. Chow. Foundations of Aerodynamics: Bases of Aerodynamic Design. John Wiley \& Sons, Inc., 5 edition, 1998.

G.V. Lachmann. Boundary Layer Control. J. R. Aero. Soc., 59(531):163-198, 1955. xix, 8, $9,10,11,12,19,20,21,22,42,105,107,121$

C.L. Ladson and C.W. Brooks, Jr. Development of a Computer Program to Obtain Ordinates for NACA 6- and 6A-Series Airfoils. TM-X 3069, NASA, 1974. 95

G.H. Lee. Laminarised All-Wing Aircraft - The HP117 Proposal. Unpublished Handley Page Report, 1961. xix, xxvii, 10, 11, 26, 27, 35, 40, 41, 42, 43, 132

R.H. Liebeck. Design of the Blended Wing Body Subsonic Transport. AIAA Journal of Aircraft, 41:10-25, 2004. xix, 23, 24

L.M. Mack. On the Stability of the Boundary Layer on a Transonic Swept Wing. AIAA-790264, 1979. 93

W.A. Mair and D.L. Birdsall. Aircraft Performance. Cambridge University Press, 1992. 58, $59,170,171,200$

R. Martinez-Val. Flying Wings. A New Paradigm for Civil Aviation. Acta Polytechnica, 47 (1), 2007. xix, 23, 24, 25, 26, 205

R. Martinez-Val and E. Perez. Optimum Cruise Lift Coefficient in Initial Design of Jet Aircraft. Journal of Aircraft, 29(4), 1991. 125, 126

R. Martinez-Val, E. Perez, P. Alfaro, and J. Perez. Conceptual Design of a Medium Size Flying Wing. Proc. IMechE, 221(Part G: J. Aerospace Engineering), 2006. 3, 134, 142

E.S. Menon. Piping Calculations Manual. McGraw Hill, 2004. 114

R.C. Nelson. Flight Stability and Automatic Control. McGraw-Hill, 1998. 146, 148, 162

K. Nickel and M. Wohlfart. Tailless Aircraft in theory and practice. Butterworth-Heinemann, 1994. xx, 3, 28, 29, 30, 31, 32, 33, 34, 35, 37, 123 


\section{REFERENCES}

M.C. Niu. Airframe Structural Design. Conmilit Press Ltd, 1988. 79, 182

J.K. Northrop. The Development of All-Wing Aircraft. The Royal Aeronautical Society, 1957. $28,29,30,31,32,36,37$

R.C. Pankhurst and N. Gregory. Power Requirements for Distributed Suction for Increasing Maximum Lift. Tech. Rep. C.P. No. 82, A.R.C., 1952. 100

W.E. Pearce. Evaluation of Laminar Flow Control System Concepts for Subsonic Commercial Transport Aircraft. Cr, NASA, 1983. 19, 20, 100, 105

W. Pfenninger. Laminar Flow Control, Laminarization. In Special Course on Drag Reduction, number AGARD-R-654, 1977. 19

W. Pfenninger. Long-range lfc transport. Technical Report N-12508, NASA, 1987. 22

P.P. Polentz. Comparison of the aerodynamic characteristics of the NACA0010 and 0010-64 airfoil sections at high subsonic Mach numbers. RM A9G19, NACA, 1949. 87, 201

D.I.A. Poll. Transition in the infinite swept attachment-line boundary layer. The Aeronautical Quarterly, pages 607-629, 1979. 16

D.I.A. Poll, M. Danks, and B.E. Humphreys. The Aerodynamic Performance of Laser Drilled Sheets. 1st European Forum on Laminar Flow Technology, pages 247-277, 1992. Hamburg, Germany. 106

R.W. Pratt. Flight Control Systems: practical issues in design and implementation. Institution of Engineering and Technology, 2000. 147

D.P. Raymer. Aircraft Design: A Conceptual Approach. AIAA Education Series, 1999. xxv, $45,47,52,55,56,59,66,67,136,161,189,191,193,195,196$

H.L. Reed and W.S. Saric. Stability of Three-Dimensional Boundary Layers. Annual Review of Fluid Mechanics, 21:235-284, 1989. 15

J.L. Rioual, P.A. Nelson, P. Hackenberg, and O.R. Tutty. Optimum Drag Balance for Boundary-Layer Suction. Journal of Aircraft, 33(2), 1996. 97

C. Rohrbach, F.B. Metzeger, D.M. Black, and R.M. Ladden. Evaluation of Wind Tunnel Performance Testings of an Advanced 45-deg Swept Eight-Bladed Propeller at Mach Numbers from 0.45 to 0.85 . CR 3505, NASA, 1982. xx, 59, 60 
H.I.H Saravanamuttoo. Modern Turboprop Engines. Pro. Aerospace Sci., 24:225-248, 1987. 176,180

W. Saric and H. Reed. Stability, Transition, and Control of Three-Dimensional Boundary Layers on Swept Wings. In IUTAM Symposium on One Hundred Years of Boundary Layer Research, pages 177-188. Springer, 2006. 14, 16

A.S.W. Thomas and K.C. Cornelius. Investigation of a Laminar Boundary-Layer Suction Slot. AIAA Journal, 20(6), 1982. 106

E. Torenbeek. Synthesis of subsonic airplane design. Delft University Press, 1976. xx, 47, $50,52,53,54,55,56,57,58,74,80,88,125,141,154,155,156,161,193,194,199,202$

E. Torenbeek. Aerodynamic Performance of Wing-Body Configurations and the Flying Wing. SAE Technical Paper, (911019):158-163, 1991. 3

G.W.H. van Es. Rapid Estimation of the Zero-Lift Drag Coefficient of Transport Aircraft. AIAA Journal of Aircraft, 39(4), 2002. 193

J. Viken and R.D. Wagner. Design Limits of Compressible NLF Airfoils. AIAA-91-0067, 1991. 93

T.A. Weishaar and H. Ashley. Static Aeroelasticity and the flying wing. Journal of Aircraft, 10(10), 1973. 23, 37, 189

R.A.L Wilson. The introduction of laminar flow to the design and optimisation of transport aircraft. PhD thesis, Cranfield University, 1997. 22, 169

A.D. Young. Boundary Layers. AIAA Education Series, 1989. 7, 52, 93, 98 